

\section{ADSORPTION MATERIALS FOR THE RECOVERY AND SEPARATION OF BIOBASED MOLECULES}


Promotion committee:

Prof. Dr. Ir. J.W.M. Hilgenkamp (Chairman)

University of Twente

Prof. Dr.-Ing. M. Wessling (Promotor)

RWTH Aachen University

Prof. Dr. Ir. D.C. Nijmeijer (Co-promotor)

University of Twente

Dr. Ir. W.M. de Vos

University of Twente

Prof. Dr. Ir. N.E. Benes

University of Twente

Prof. Dr. Ir. R.G.H. Lammertink

University of Twente

Prof. Dr.-Ing. A. Jupke

RWTH Aachen University

Prof. Dr.-Ing. M.H.M Eppink

WUR Wageningen

This work was carried out within project SC-00-04 of the Institute for Sustainable Process Technology (ISPT), The Netherlands.

Adsorption materials for the recovery and separation of biobased molecules

ISBN: 978-90-365-4183-1

DOI: $10.3990 / 1.9789036541831$

URL: http://dx.doi.org/10.3990/1.9789036541831

Cover design by CGR-ARTWORKS

Printed by: Ipskamp drukkers, Enschede

CCopyright 2016 A.C. IJzer 


\title{
ADSORPTION MATERIALS FOR THE RECOVERY AND SEPARATION OF BIOBASED MOLECULES
}

\author{
Dissertation
}

\author{
to obtain \\ the degree of doctor at the University of Twente, \\ on the authority of the rector magnificus, \\ prof. dr. H. Brinksma, \\ on account of the decision of the graduation committee, \\ to be publicly defended \\ on Friday $2^{\text {nd }}$ of September at 12:45 \\ by \\ Anne Corine IJzer \\ Born on $22^{\text {nd }}$ of August 1979 \\ in Enschede, the Netherlands
}


This thesis has been approved by:

Prof. Dr.-Ing. M. Wessling (Promotor)

Prof. Dr. Ir. D.C. Nijmeijer (Co-promotor) 
Opgedragen aan mijn ouders Ank en Reinier IJzer 



\section{Table of contents}

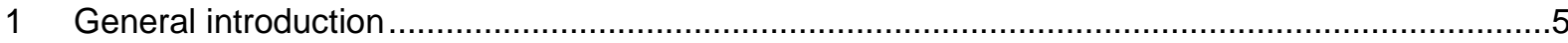

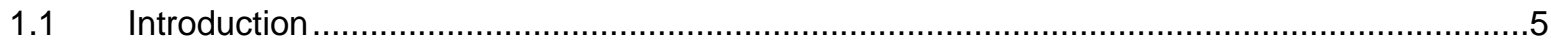

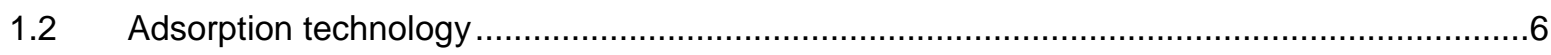

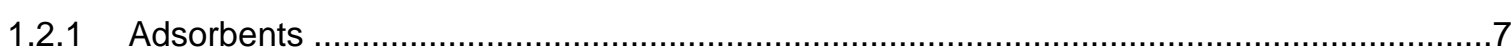

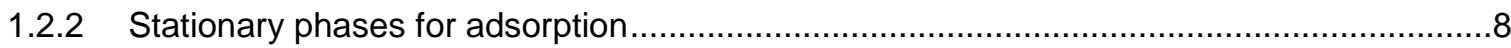

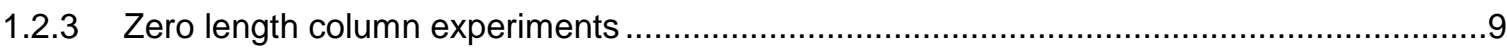

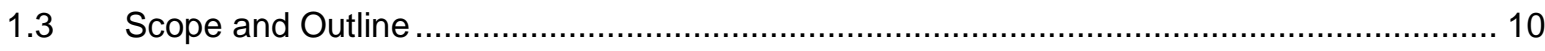

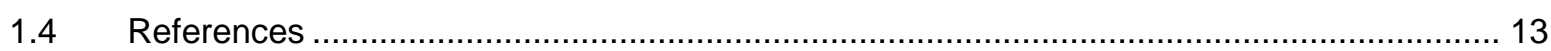

2 Adsorption technology and alternative stationary phases for bioprocessing, theoretical

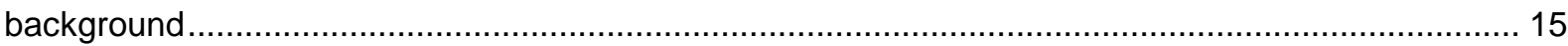

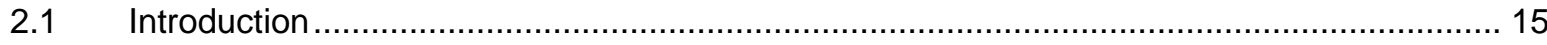

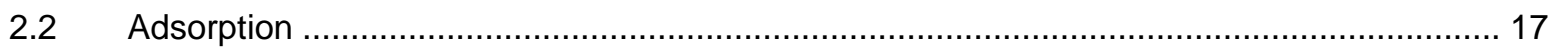

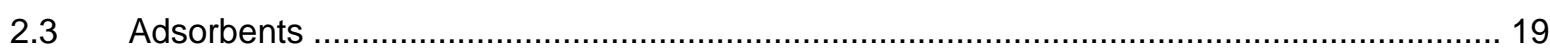

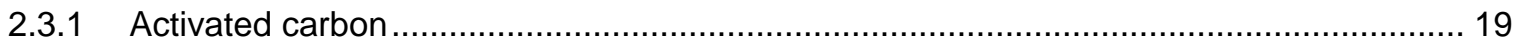

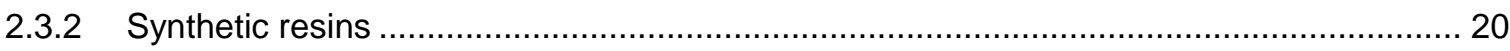

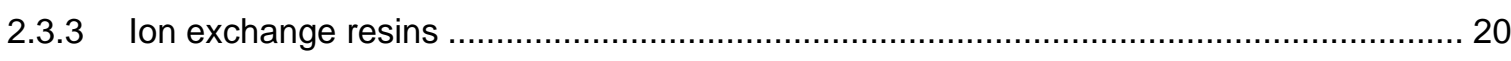

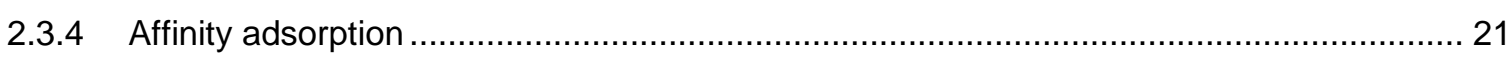

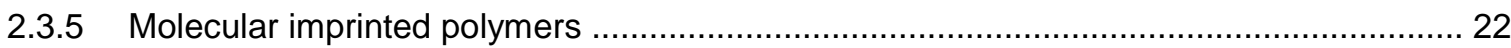

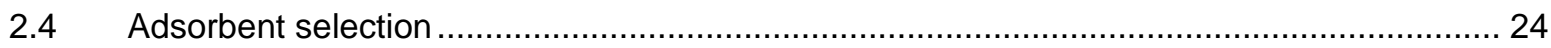

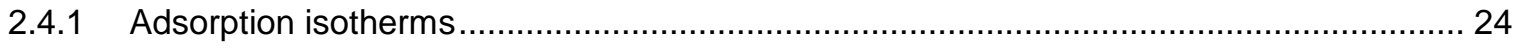

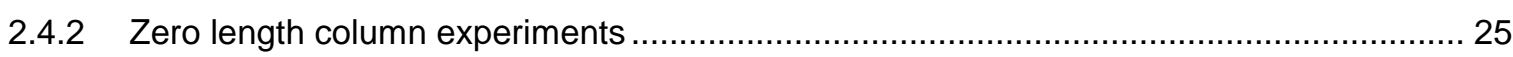

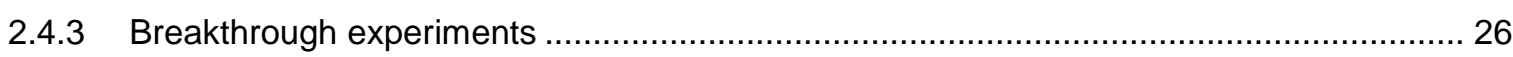

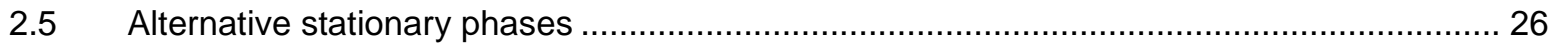

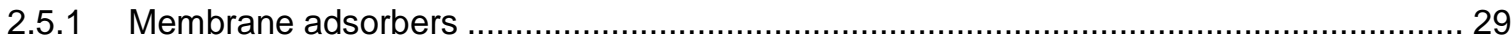

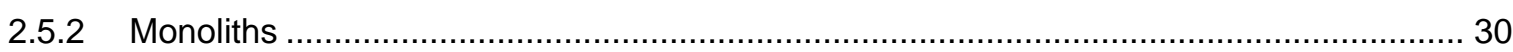

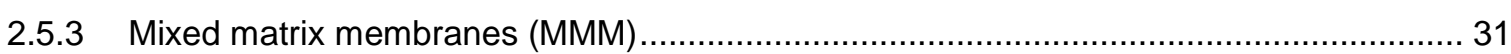

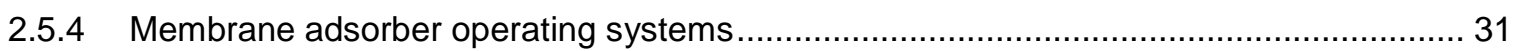

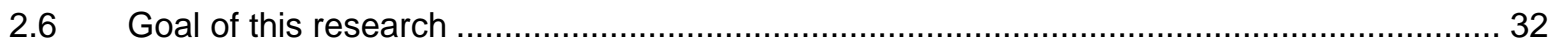

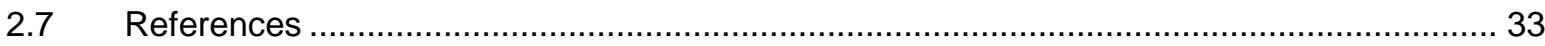

3 Performance analysis of aromatic adsorptive resins for the effective removal of furan derivatives from glucose. 


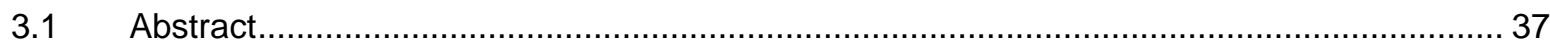

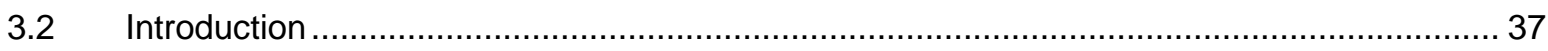

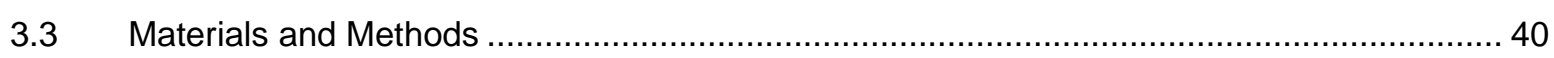

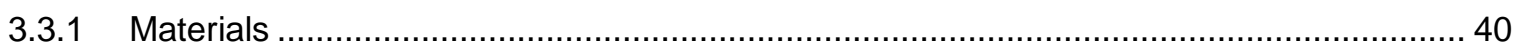

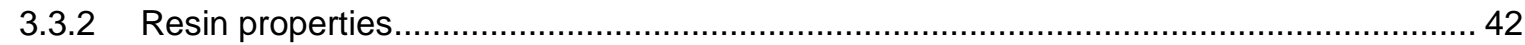

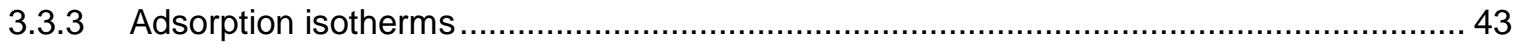

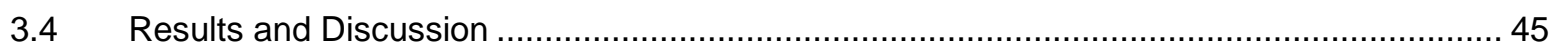

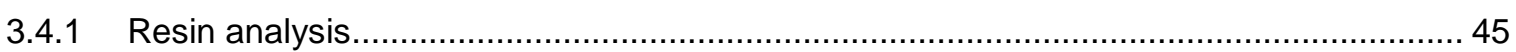

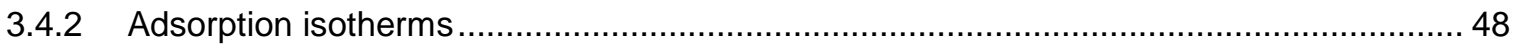

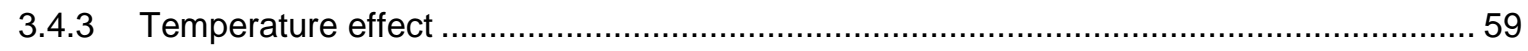

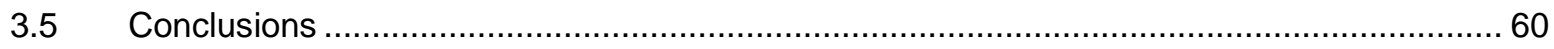

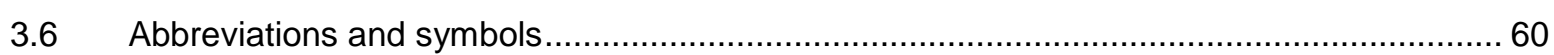

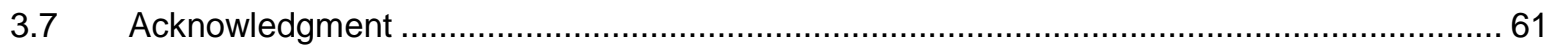

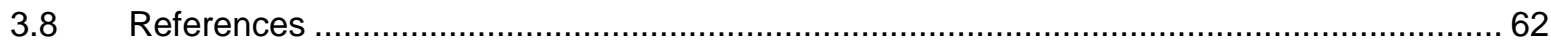

4 Adsorption kinetics of Dowex $^{\text {TM }}$ Optipore $^{\text {TM }}$ L493 for the removal of furan 5-

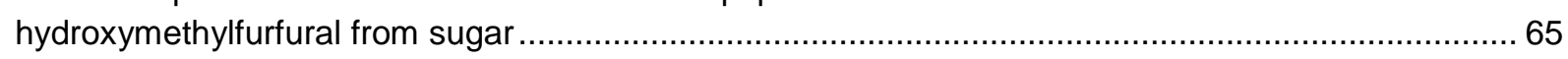

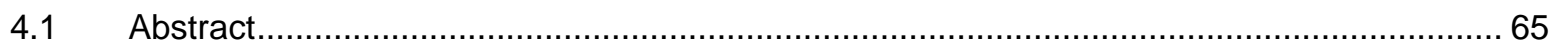

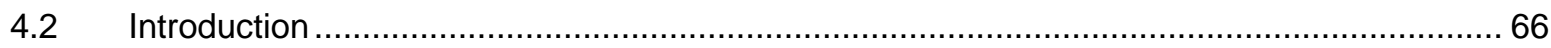

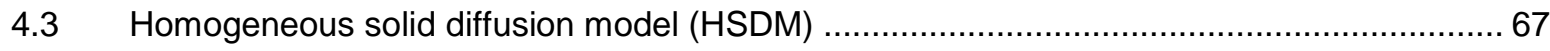

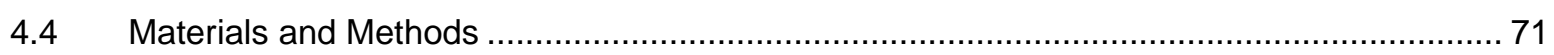

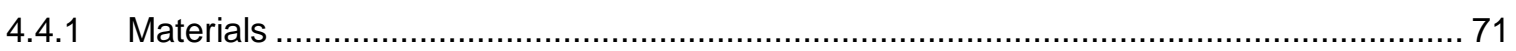

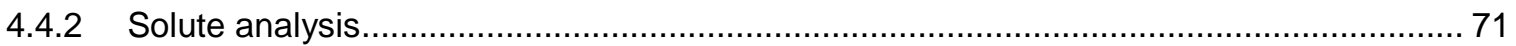

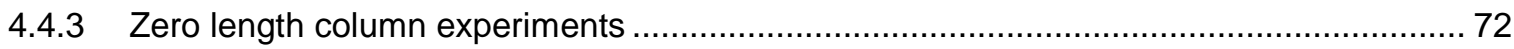

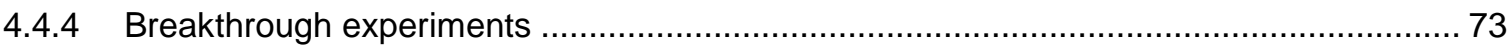

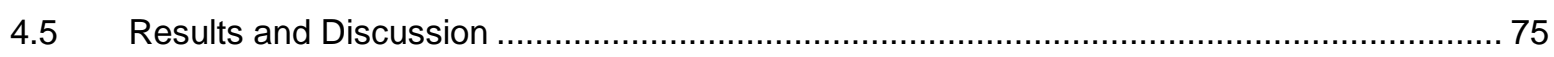

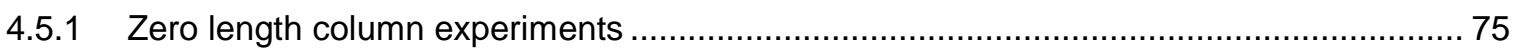

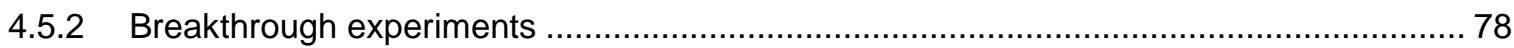

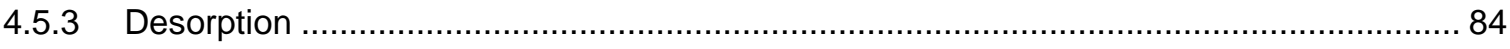

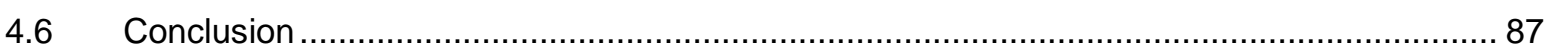

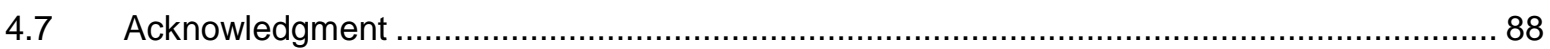

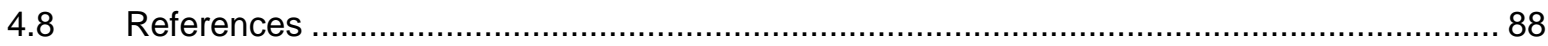

5 Very fast adsorption of biological anions by particle loaded mixed matrix membranes ................ 90

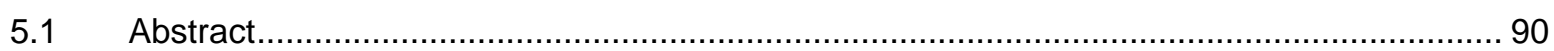

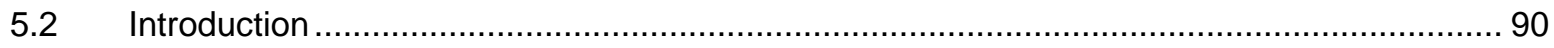

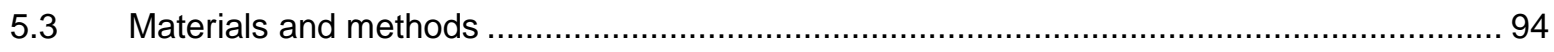

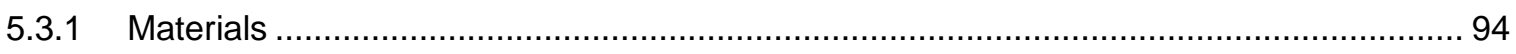

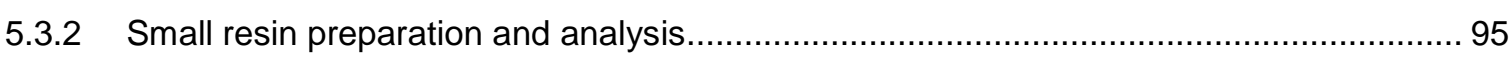




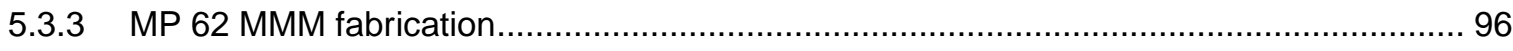

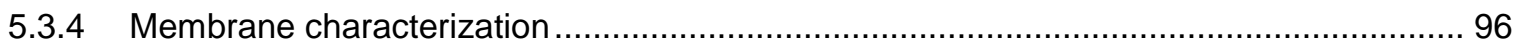

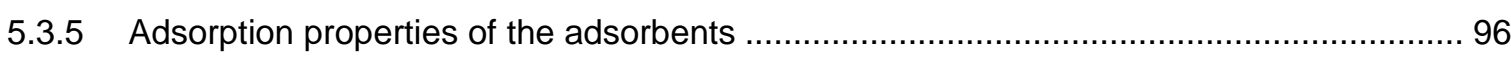

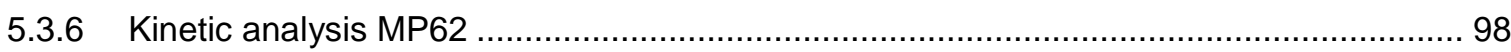

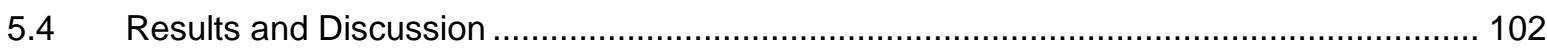

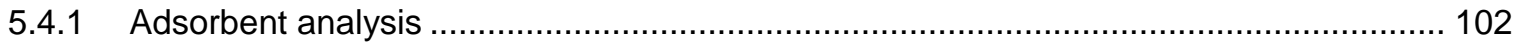

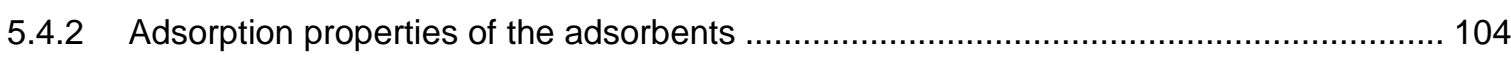

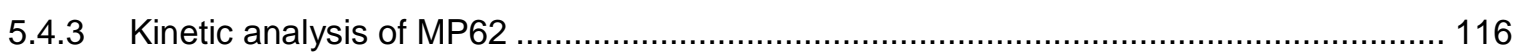

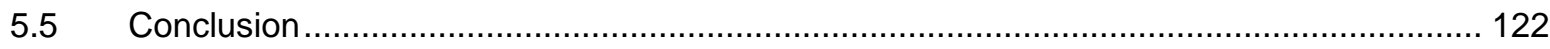

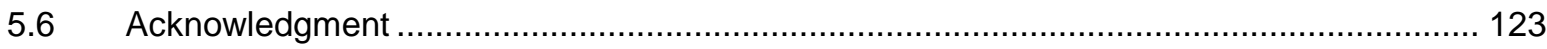

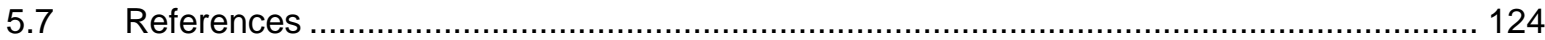

6 Particle loaded mixed matrix membranes for high throughput HMF adsorption applications ..... 127

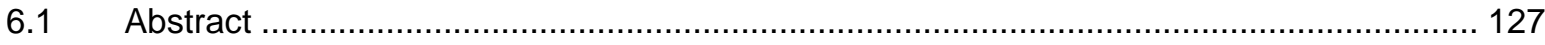

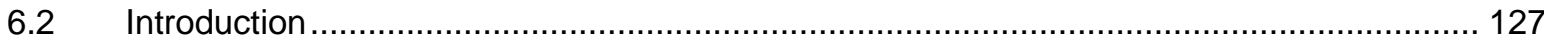

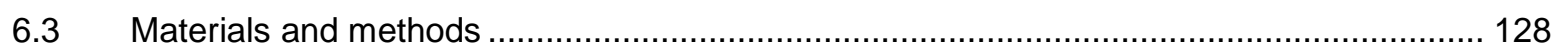

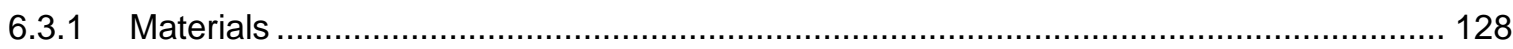

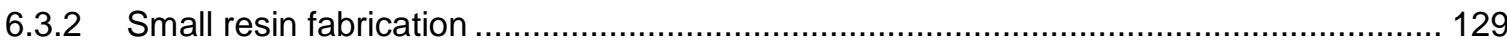

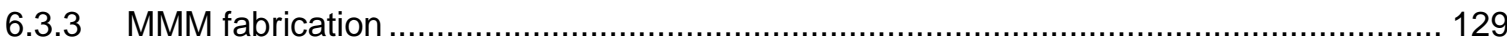

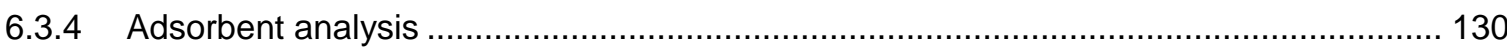

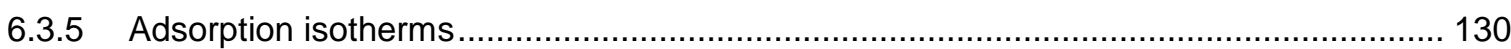

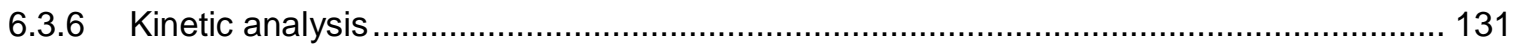

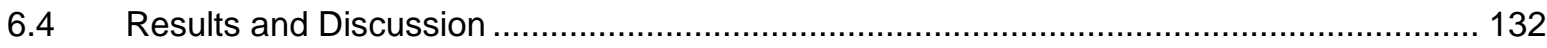

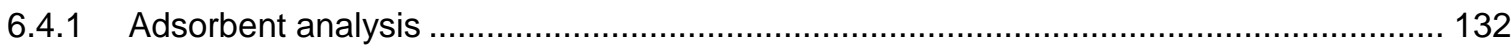

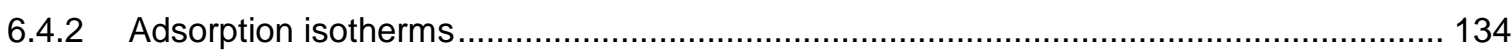

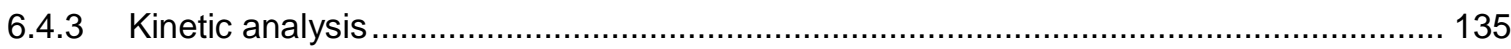

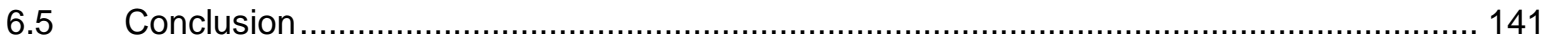

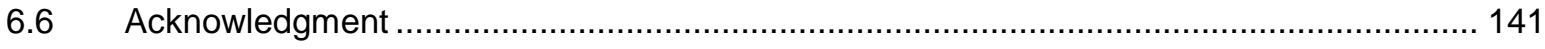

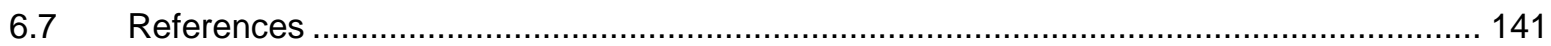

7 Reaction media for molecularly imprinted membranes for acid removal applications ................. 143

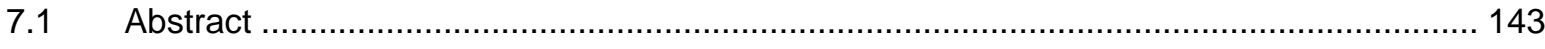

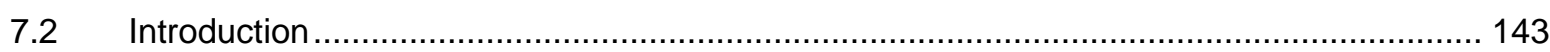

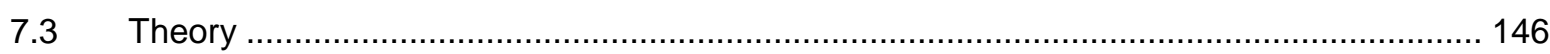

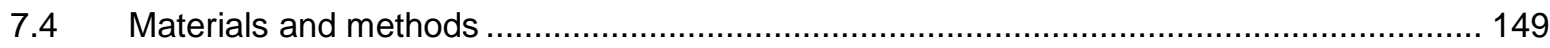

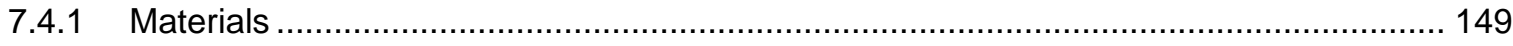

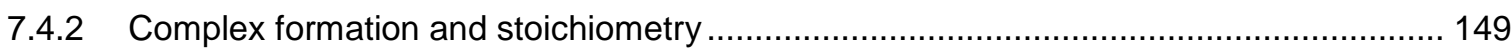

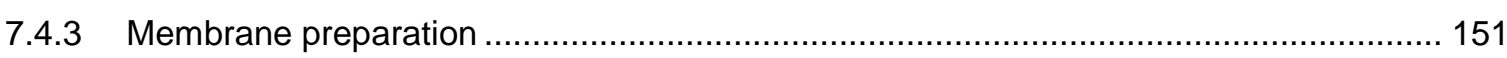




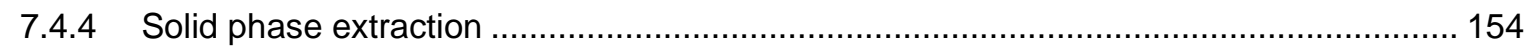

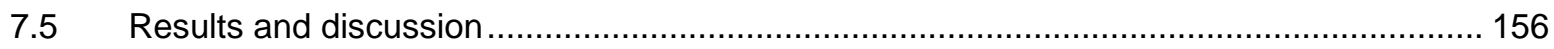

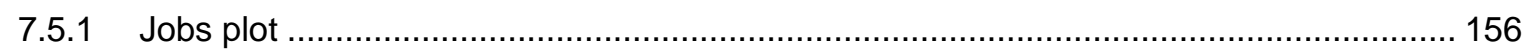

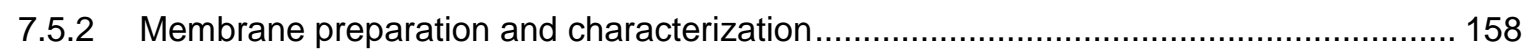

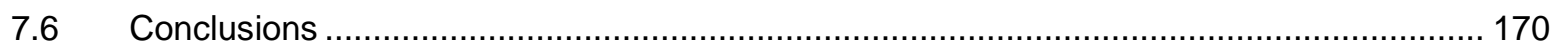

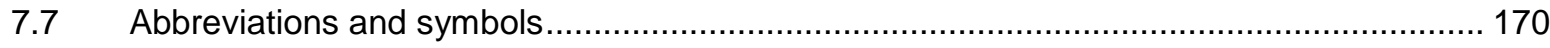

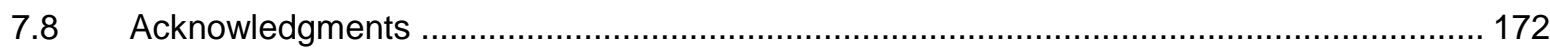

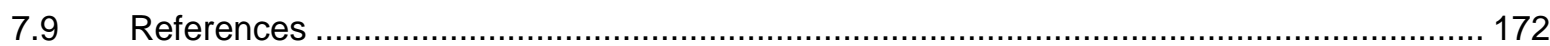

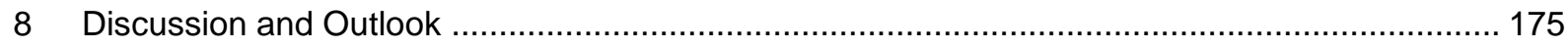

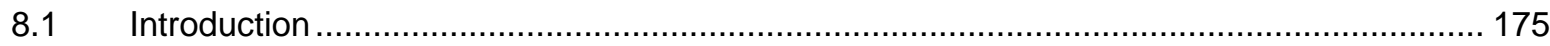

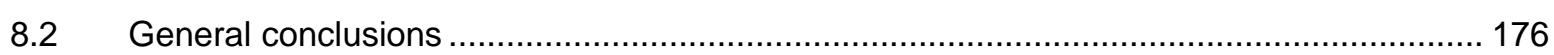

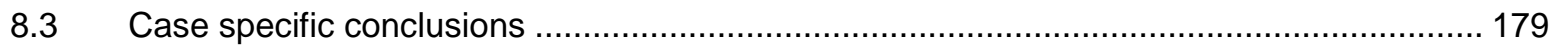

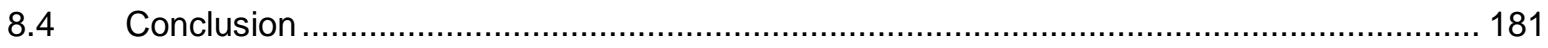

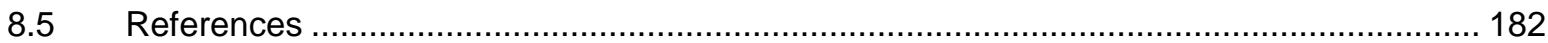

Appendix: Particle loaded MMMs for resins with poor kinetic adsorption properties ........................ 183

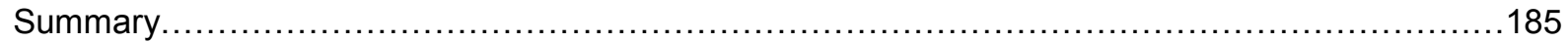

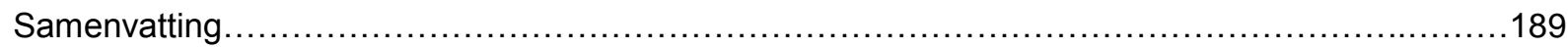




\section{General introduction}

\subsection{Introduction}

Since in the 70's biotechnology companies emerged, they are nowadays recognized as one of the most booming areas of industry (see NASDAQ Biotechnology index Figure 1) [1-7]. Biotechnology companies produce products from biological origin, the bioproducts are harvested from cells of plant tissue or cells grown in bioreactors.

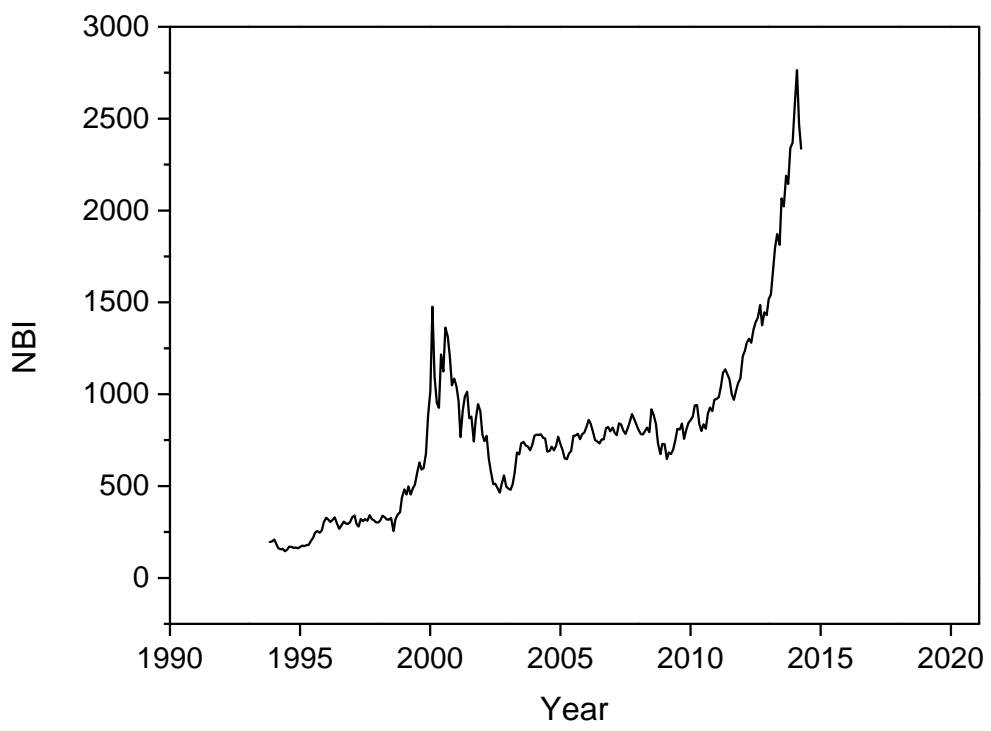

Figure 1: NASDAQ Biotechnology Index (NBI) [8]

A large part of the cost of the bioproducts is based on the bioseparations in the downstream processing which is necessary to concentrate and purify the bioproducts. Separations of bioproducts are often expensive and/or difficult because bioproducts are usually present in low concentrations in the starting material, have similar hard to separate byproducts, have stringent quality or purity demands and are susceptible to degeneration [9]. These properties lead to multistep separations with techniques that show tradeoffs between high selectivity (or resolution) for purification and high throughput (or productivity) for concentration of the product. 
In this thesis we aim for cheaper, more sustainable and environmentally friendly bioproducts by evaluating and improving the work horse of bioseparations: Adsorption technology.

\subsection{Adsorption technology}

Adsorption technology, especially chromatography, is regarded as the workhorse of bioseparations

$[10,11]$. Adsorption is the process of concentrating molecules (solutes) on the surface of the adsorbent. It typically consist of four steps (Figure 2): In the loading step the feed solution passes the adsorbent, adsorption of the target solute occurs, the feed solution is withdrawn in the washing step and finally the adsorbed target solute is removed from the adsorbent. This last step is omitted if the adsorbent is discarded instead of regenerated.
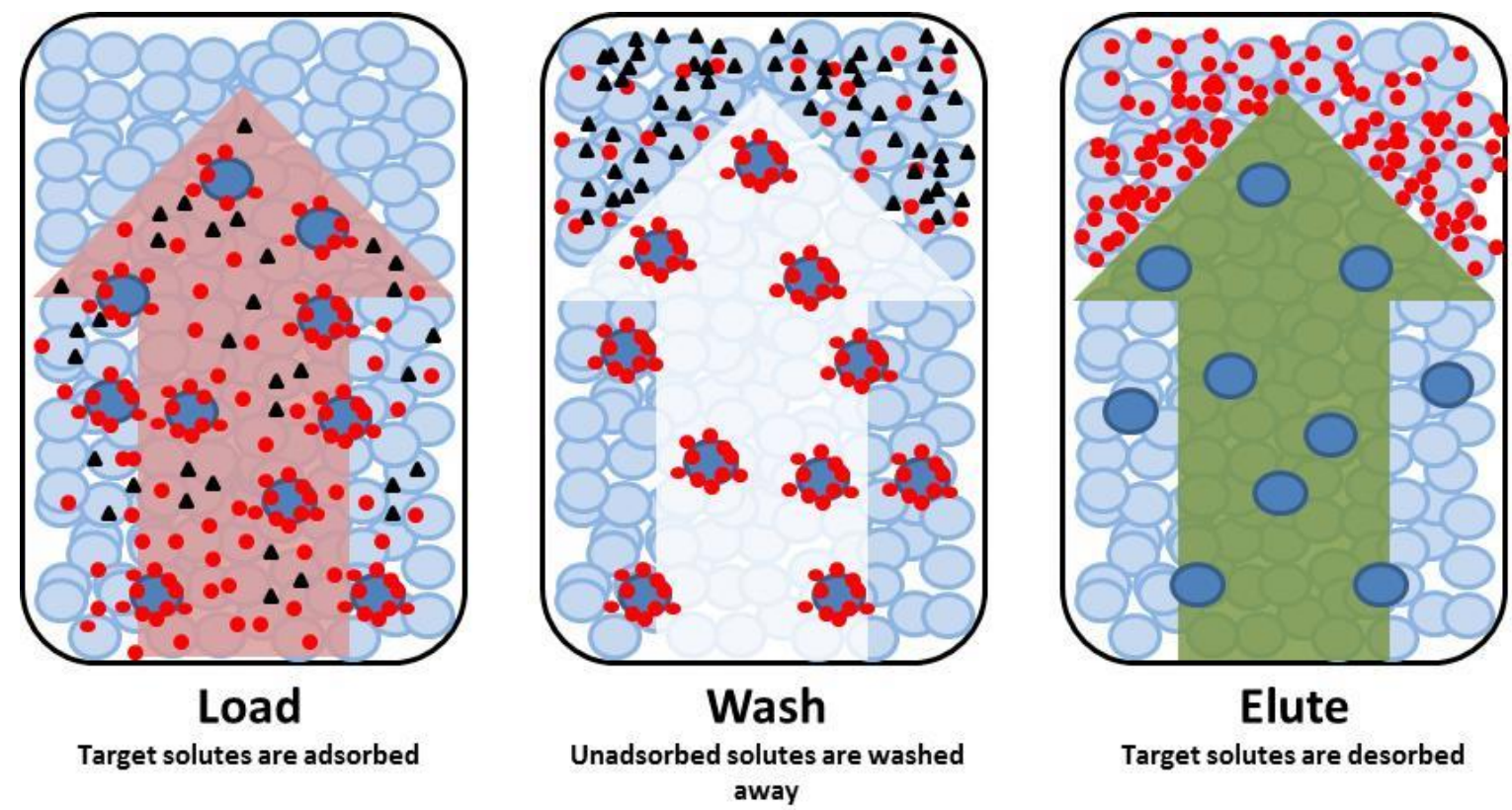

Figure 2: Schematic representation of adsorption technology.

The main technologies for bioseparations based on adsorption technology are fixed bed adsorption, expanded bed adsorption, simulated moving bed adsorption, and liquid chromatography [12]. 


\subsubsection{Adsorbents}

The effectiveness of adsorption technology is firstly based on the adsorbent; It's capacity, selectivity, regenerability, adsorption kinetics, compatibility with the solutes and costs [12]. The capacity determines how much solute the adsorbent adsorbs, while the selectivity of the adsorbent for the target solute determines the purity of the product. The regenerability is determined by the amount and type of solvent and the temperature required to desorb the solute from the adsorbent. Adsorption kinetics determine the time necessary for adsorption (and desorption).

In industry a wide variety of adsorbents are used for bioseparations, usually based on carbons or synthetic resins [13]. The main advantages of activated carbons are that they are cheap and available in many different pore sizes with high surface areas, unfortunately regeneration is often difficult. Synthetic resins are very suitable as adsorbents because the properties (physical and chemical) can be adapted to fit the adsorption application. A big improvement made with synthetic resins has been the development of hypercrosslinked resins [14]. Hypercrosslinked resins are produced by post-crosslinking polymers chains or macroporous resins in solution or in swollen state [14-16]. This method produces a highly crosslinked polymer network with low packing density which is able to swell in polar and non-polar media. The resin shows superiority due to their higher surface area and uniform pore size, making high adsorption capacities and good adsorption kinetics possible.

The separation of furans from sugar is often investigated (e.g [17-21]). Different resins were described as good resins for these type of separations. However it was impossible to determine which resin was best and why because a systematic comparison of the different resins based on adsorption capacity, selectivity and kinetic properties was not performed. In this thesis we therefore compared different styrene based resins and determined the key properties of the resins for the separation of the furan 5 hydroxymethylfurfural (HMF) from glucose (Chapter 3). Furthermore we investigate the properties of a hypercrosslinked adsorbent (Chapter 3 and Chapter 4) for the separation of HMF from glucose.

A relative new method to improve the selectivity of an adsorbent is by molecular imprinting of a polymer (MIP). Molecular imprinting of polymers is a technique to create adsorbers with tailored adsorption sites for specific molecules. To accomplish this, MIPs contain functional groups, which 
are processed or polymerized in the presence of a template molecule (Figure 3) [22]. The polymer binding site, formed by these specific functional groups, is ordered in a three dimensional structure that makes the polymer also shape selective. This makes these molecularly imprinted polymers (MIPs) comparable with biological processes such as ligand-receptor binding.

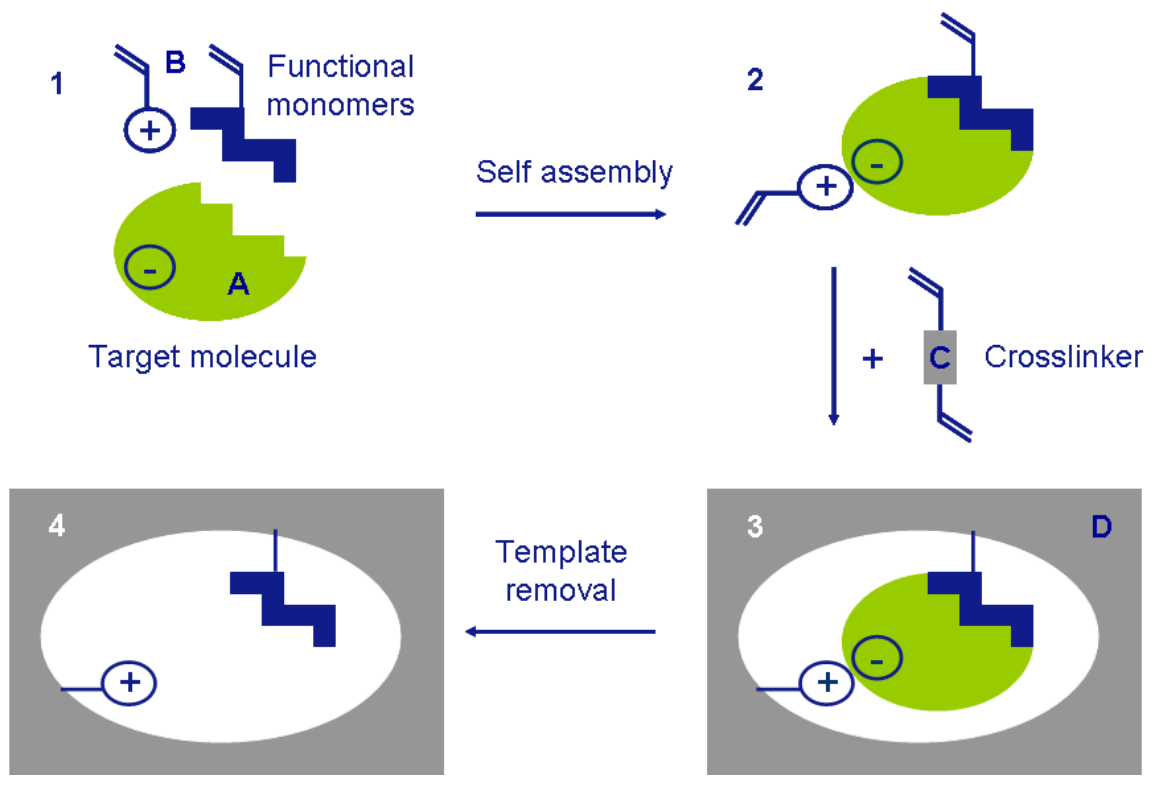

Figure 3: Illustration of the molecular imprinting process: 1: mixing of target molecule or template and reactants; 2 : selfassembling of target molecule and reactants; 3: stabilization of target molecule by crosslinking; 4: extraction of target molecule or template finally resulting in a polymer with shape and size selective binding site (MIP).

Molecular imprinting has been recognized as an alternative for the selective separation of acids from complex mixtures. We examined the properties of the reaction mixture and adsorption mixture necessary for the molecular imprinting and recognition of salicylic acid (Chapter 7).

\subsubsection{Stationary phases for adsorption}

A packed bed is a column filled with adsorption material (Figure 4). It is the most common and simple stationary phase for adsorption. However it has some downsides. It shows high pressure drops and the mass transfer of solutes to and from the adsorption sites is largely dependent on the slow intra particle diffusion. Packed beds are also susceptible to channeling, which is cause to the incomplete use of the column capacity. Recently the stationary phases for bioseparations have been improved by using new hybrid adsorption phases such as monoliths, membrane adsorbers or particle loaded mixed matrix membranes (Figure 4) $[9-11,23,24]$. These stationary phases consist 
of a very porous structure with interconnected pores, such as found in porous membranes, that allow for uninterrupted bulk flow. The adsorption phase is coated on the porous structure or dispersed as particles in the porous structure. The high porosity and short diffusion paths make very high flow rates possible.

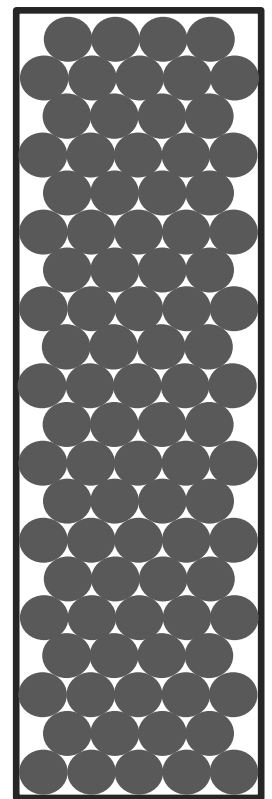

Packed bed

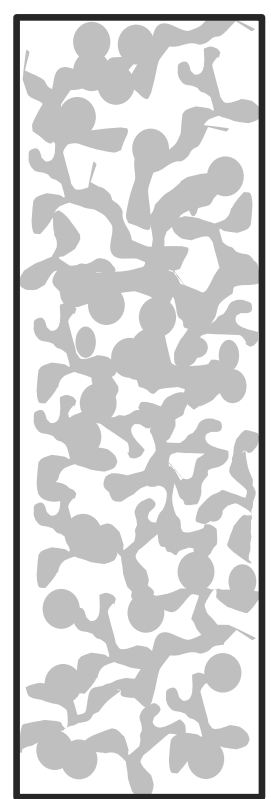

Monolith

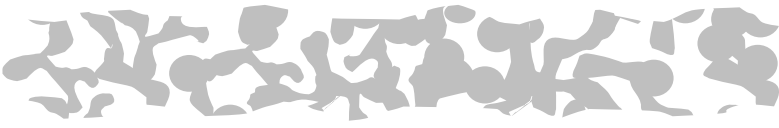

Membrane adsorber

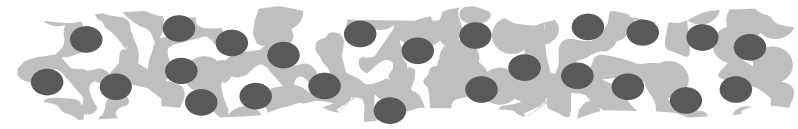

Particle loaded membrane adsorber (MMM)

Figure 4: Different stationary phases, adapted from [23].

In this thesis we fabricated MMMs with small anion exchange particles (Chapter 5) and small hypercrosslinked particles (Chapter 6). These MMMs were studied on capacity and kinetic properties.

\subsubsection{Zero length column experiments}

There are different experimental set-ups that can be used to determine adsorption kinetics of adsorbents. The zero length column (ZLC) introduced by Eic and Ruthven in 1988 is frequently used to study the adsorption kinetics of adsorbents [25]. In this experiment, a solute is pumped from a vessel over a very short column containing the adsorbent back to the vessel (Figure 5). The short bed length, the flow rate and the solution volume are chosen in such way that the 
concentration of the solution is almost equal in the entire set up at a given time, mimicking an ideal mixed finite bath. This method is also very suitable to measure the adsorption kinetics of MMMs when the column is replaced by a filter holder with the MMM under investigation. The fast flow through the membrane ensures maximal convective flow through the pores of the membrane minimizing solute transport time by slow diffusion through the membrane pores.

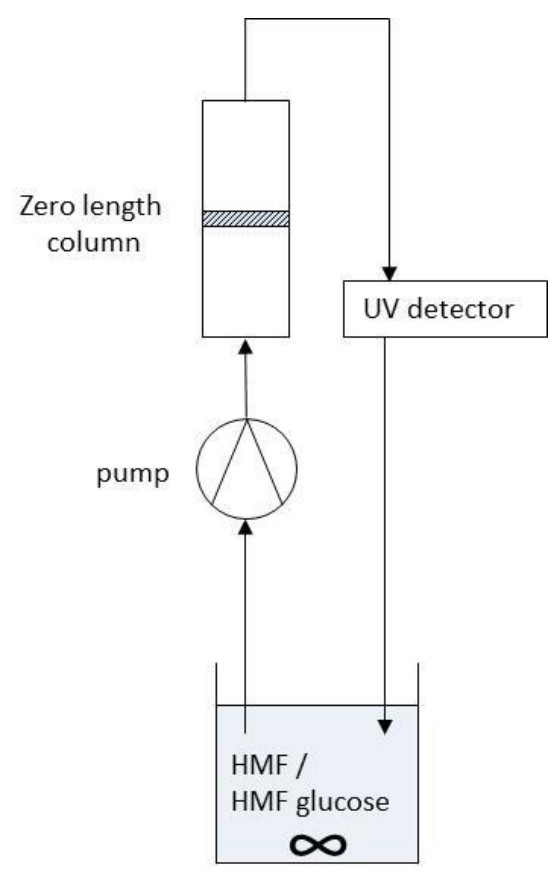

Figure 5: Schematic representation of experimental zero length column (ZLC) set up.

In this thesis we used the ZLC column to study the effect of glucose on diffusion (Chapter 3). Furthermore we used the experiment to study the effect of incorporating small particles into MMMs (Chapter 4 and 5). There is very limited literature available on ZLC combined with membranes and to our knowledge it is the first time that ZLC experiments are applied while membranes themselves function as a column, ensuring convective flow through the pores of the membrane.

\subsection{Scope and Outline}

The scope of this thesis is to improve adsorption processes by means of:

1. Selecting the best adsorbent, based on selectivity, capacity and adsorption kinetics. 
2. Improving adsorption performance of adsorbents by incorporation of small adsorbents in an alternative stationary phase: a particle loaded mixed matrix membrane.

3. Improving the selectivity of the adsorbent by molecular imprinting.

In Chapter 2: "Adsorption technology and alternative stationary phases for bioprocessing" explains the basics of bioseparations for the bioprocessing industry. The importance of downstream processing for a viable biotechnology is stressed and new directions for improved bioseparations are discussed. These include alternative stationary adsorption phases such as monoliths, membrane adsorbers and particle loaded mixed matrix membranes. Furthermore improved adsorption materials such as hypercrosslinked adsorbers with improved adsorption capacity and faster kinetics and molecularly imprinted materials that show very high selectivity are discussed. The different experiments available for adsorbent investigation are also discussed.

In Chapter 3: "Performance analysis of aromatic adsorptive resins for the effective removal of furan derivatives from glucose" the adsorption performance of styrene based resins for the separation of 5-hydroxymethylfurfural (HMF) from glucose was investigated. Adsorption experiments with 5 different resins were performed to determine the relation between adsorption properties and chemical and physical material properties. Dowex ${ }^{\mathrm{TM}}$ Optipore $^{\mathrm{TM}}$ L493 (Optipore) was identified as the best performing resin based on adsorption capacity and competitive adsorption experiments due to its high surface area.

In Chapter 4: "Adsorption kinetics of Dowex ${ }^{\mathrm{TM}}$ Optipore $^{\mathrm{TM}}$ L493 for the removal of the furan 5hydroxymethylfurfural from sugar" the kinetic adsorption properties of Optipore were investigated. Optipore is a hypercrosslinked polymer and is therefore known for its high surface area as well as good interconnected pores. In Chapter 3 we have already shown this lead to excellent HMF adsorption capacities. In this chapter we show with zero length column experiments (ZLC) as well as breakthrough experiments that this resin also outperforms its competitors based on kinetic performance.

In Chapter 5: "Very fast adsorption of biological anions by particle loaded mixed matrix membranes" the adsorption capacities and kinetic properties of anion exchange mixed matrix membranes (MMMs) are examined and compared with conventional large particles. Small, medium and large biological anions were used for these experiments. The adsorption capacity of the resins 
in the MMMs is hardly affected by incorporation into the MMMs. The ZLC experiments show that the effective diffusion coefficient is larger for the MMMs than for the large particles due to the lengthening of the diffusion path due to some of the pores of the membranes. However the overall adsorption time of the MMMs is much faster making MMMs good candidates for high throughput applications.

The MMMs in the previous chapter showed great potential for the high throughput applications of small molecules. In Chapter 6: "Particle loaded mixed matrix membranes for high throughput 5hydroxymethylfurfural adsorption applications" newly fabricated Optipore MMMs were investigated for the removal of 5-hydroxymethylfurfural (HMF). The ZLC experiments show that the pores of the membranes offer less resistance than the pores of the MMMs examined in Chapter 5. Again the overall adsorption time of the MMMs is reduced enormously compared to that of the large resins. The breakthrough experiments performed with the MMMs show that the MMMs are indeed capable of handling much higher flow rates than the conventional adsorption columns with large particles. In contrast to conventional columns, the adsorption capacity even improves when higher flow rates are used. The adsorption performance per gram resin is much better for MMMs than for the original large resins.

Biological mixtures often have by-products and other impurities that have similar chemical and physical properties as the target molecule. These mixtures require very specific separation techniques. In Chapter 7: "Reaction media for molecularly imprinted membranes for selective acid adsorption from aqueous media" we tried to make very selective adsorbers for small anions by molecular imprinting. Molecular imprinting of molecules for aqueous (biological) applications is difficult because often organic solutions are necessary for the imprinting resulting in complexes that differ from the possible complexes formed in the aqueous application. With the continuous variation method we identified the critical parameters for imprinting the small acidic molecule salicylic acid. It showed that $\mathrm{pH}$ and solvent composition are essential for successful molecular imprinting for aqueous applications

Chapter 8 summarizes and discusses the findings of these thesis. The applied methods and results are critically discussed and an outlook on the direction of future research on adsorption is given. 


\subsection{References}

1. Massoudi, A. and M. Mackenzie, US biotech stocks soar to new record high, in ft.comFebruari 12, 2014: New York.

2. Hay, T., Biotechnology boom is here to stay, in Wall Street Journal DigitalOctober 14, 2013.

3. Alter, D., How to invest in biotech now and double your money. Money Morning, June 19, 2013.

4. Handbook of Bioseparations, ed. S. Ahuja. 2000, San Diego: Academic Press.

5. Harrison, R.G., et al., Bioseparation Science and Engineering, ed. K.E. Gubbins. 2003, New York: Oxford University Press.

6. Huang, H.J., et al., A review of separation technologies in current and future biorefineries. Separation and Purification Technology, 2008. 62(1): p. 1-21.

7. Hughes, B. and L.E. Hann, The production of biopharmaceuticals, in Biologics in General Medicine. 2007, Springer. p. 59-66.

8. Market Watch, NASDAQ Biotechnology Index. [cited 2014 May 1].

9. Ghosh, R., Principles of bioseparations engineering. 2006, Singapore: World Scientific Publishing Co. Pte. Ltd.

10. Ghosh, R., Protein separation using membrane chromatography: Oportunities and challenges. Journal of Chromatography A, 2002. 952(1-2): p. 13-27.

11. Przybycien, T.M., N.S. Pujar, and L.M. Steele, Alternative bioseparations operations: life beyond packed-bed chromatography. Current Opinion in Biotechnology, 2004. 15(5): p. 469-478.

12. Thomas, W.J. and B. Crittenden, Adsorption Technology and Design. 1998: Butterworth Heinemann.

13. Belter, P.A., E.L. Cussler, and W.-s. Hu, Bioseparations, downstream processing for biotechnology. 1988, New York: John Wiley \& Sons.

14. Xu, Z., Q. Zhang, and H.H.P. Fang, Applications of Porous Resin Sorbents in Industrial Wastewater Treatment and Resource Recovery. Critical Reviews in Environmental Science and Technology, 2003. 33(4): p. 363-389.

15. Tsyurupa, M.P. and V.A. Davankov, Porous stucture of hypercrosslinked polystyrene: State-of-the-art mini-review. Reactive and Functional Polymers, 2006. 66(7): p. 678-779.

16. Jerabek, K., L. Hankova, and Z. Prokop, Post-crosslinked polymer adsorbents and their properties for separation of furfural from aqueous solutions. Reactive Polymers, 1994. 23(2-3): p. 107-112.

17. Canilha, L., et al., Bioconversion of Sugarcane Biomass into Ethanol: An Overview about Composition, Pretreatment Methods, Detoxification of Hydrolysates, Enzymatic Saccharification, and Ethanol Fermentation. Journal of Biomedicine and Biotechnology, 2012: p. 15.

18. de Carvalho, W., et al., Detoxification of sugarcane bagasse hemicellulosic hydrolysate with ion-exchange resins for xylitol production by calcium alginate-entrapped cells. Journal of Chemical Technology \& Biotechnology, 2004. 79(8): p. 863-868.

19. Larsson, S., et al., Comparison of different methods for the detoxification of lignocellulose hydrolyzates of spruce. Applied Biochemistry and Biotechnology, 1999. 77-9: p. 91-103.

20. Maciel de Mancilha, I. and M.N. Karim, Evaluation of ion exchange resins for removal of inhibitory compounds from corn stover hydrolyzate for xylitol fermentation. Biotechnology progress, 2003. 19(6): p. 1837-1841.

21. Vern, C., et al., The beet sugar factory of the future. International Sugar Journal, 1995. 97(1159): p. 310-316.

22. Yan, M. and O. Ramström, Molecularly Imprinted Materials: Science and Technology. 2004: Taylor \& Francis.

23. Orr, V., et al., Recent advances in bioprocessing application of membrane chromatography. Biotechnology Journal, 2013. 31(4): p. 450-465.

24. Tran, R., et al., Changing manufacturing paradigms in downstream processing and the role of alternative bioseparation technologies. Journal of Chemical Technology \& Biotechnology, 2013.

25. Eic, M. and D.M. Ruthven, $A$ new experimental technique for measurement of intracrystalline diffusivity. Zeolites, 1988. 8(1): p. 40-45. 


\section{Adsorption technology and alternative stationary phases for bioprocessing, theoretical background}

\subsection{Introduction}

Since in the 70's biotechnology companies emerged, they are nowadays recognized as one of the most booming areas of industry (see NASDAQ Biotechnology index Figure 1) [1-7]. Biotechnology companies produce products from biological origin, the bioproducts are harvested from cells of plant tissue or cells grown in bioreactors. The products range from the small molecule methanol [8] to whole cells [9], and include industrial and commodity chemicals [8, 10, 11], biopharmaceuticals [7, 12-14], food and food additives [10,11, 15, 16], diagnostic products [17], cosmetic products [15, 16], nutraceuticals [12, 16] and agrochemicals [18]. In the field of biotechnology bioseparations are vitally important to obtain pure and concentrated products.

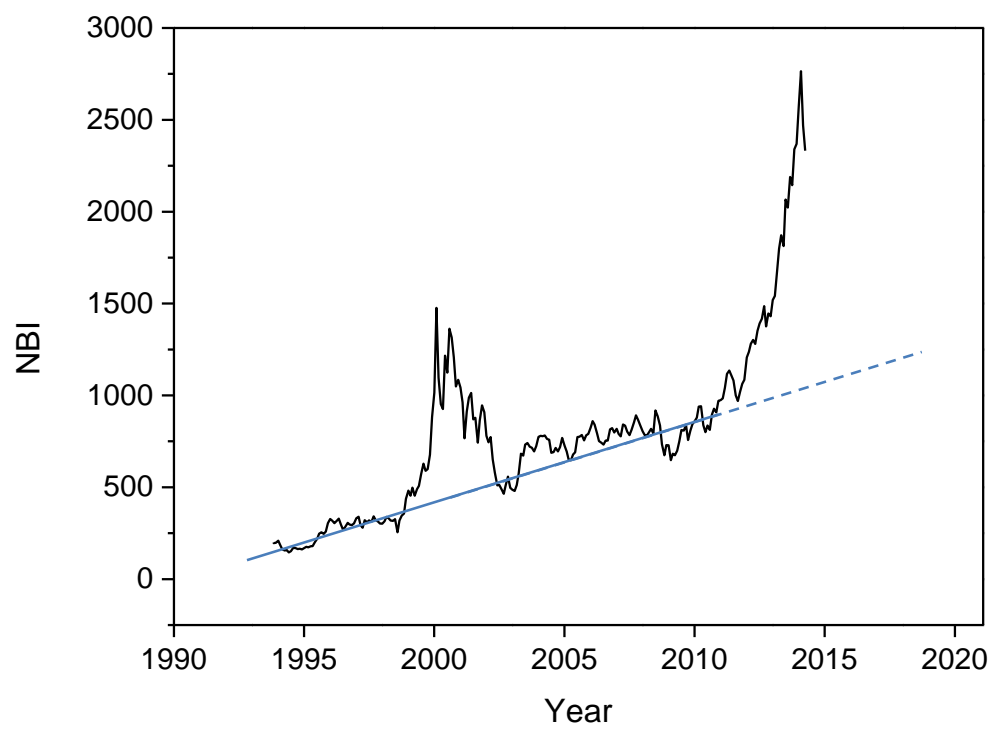

Figure 1: NASDAQ Biotechnology Index (NBI) [19]

Just like bulk commodity chemicals biological products are separated based on size, density, diffusivity, shape, polarity, solubility, electrostatic charge and volatility. However, bioseparations show some substantial differences from bulk chemistry separations [20]: 
1. Biological products are present in very low concentrations in the starting material, large volume streams need to be processed.

2. Multi technique separations are often required.

3. By-products and other impurities often have similar chemical and physical properties as the target molecule. Very specific separation techniques are required in those cases.

4. The stringent quality requirements of pharmaceuticals and diagnostic products call for a very high level of purification.

5. Biochemicals are susceptible to degeneration. The stability of the products often requires 'gentile' separations in terms of $\mathrm{pH}$, shear stress, ionic strengths, temperature and type of solvent used.

Due to these specifics, the following process scheme is usually followed in bioseparations: first the removal of insolubles (filtration, centrifugation), second isolation of the product (adsorption, solvent extraction), third purification of the product (chromatography, electrophoresis, precipitation) and finally polishing of the product (crystallization) [20, 21]. In technologies used for bioseparations there is usually a tradeoff between high selectivity (or resolution) and high throughput (or productivity). In the typical process scheme low-selectivity/high throughput techniques (e.g. precipitation, filtration, centrifugation, adsorption and crystallization) are used first for the removal of insolubles and isolation of the product, followed by high-selectivity/low throughput techniques (e.g. affinity separations, chromatography, and electrophoresis) for purification and polishing of the product. With the low-selectivity/high-throughput techniques the volume is greatly reduced thereby concentrating the products. The lower volume is further processed by high-selectivity/lowthroughput techniques to purify the products. The disadvantage of this process scheme is that the capital and operational costs are high while the product recovery is low.

A large part of the cost of the bioproducts is based on the bioseparations during the downstream processing. This is due to the low concentration of the products in the starting material. The Sherwood plot of bioproducts (Figure 2) shows that the price of the products is mostly related to the initial concentration of the product [22]. The energy involved in concentrating the products is determined by thermodynamics and can hardly be reduced, but the (complex) multi separations that are often used for the concentration and purification, reduces the efficiency of the separations 
and leave room for improvement. Recently many investigations to optimize the separations by improving and combining separation techniques have been performed. Membrane and monolith chromatography, expanded bed chromatography, high resolution ultrafiltration are examples of technologies that have improved or have the potential to improve the separation efficiency in bioprocessing $[4,20,23,24]$. All these techniques offer the possibility of high throughput combined with high selectivity.

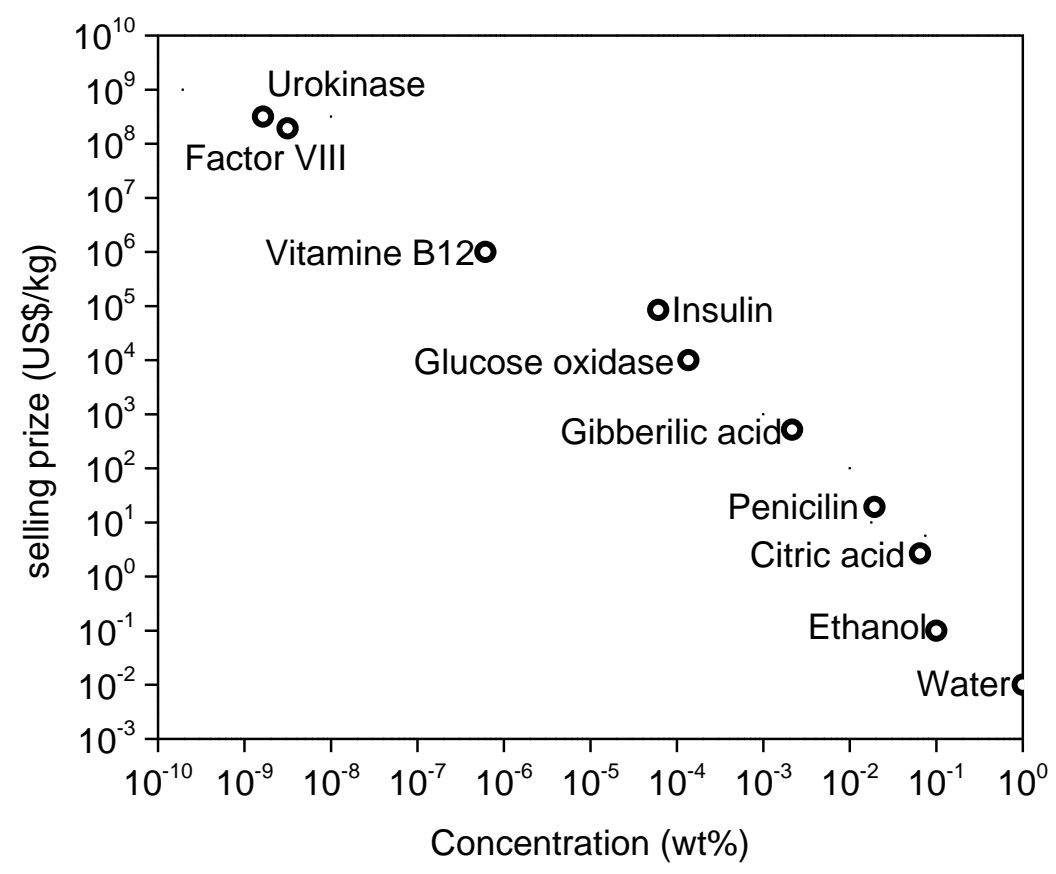

Figure 2: Sherwood plot (data 1984) adapted from [25] as reported in [22].

\subsection{Adsorption}

Adsorption technology, especially chromatography, is regarded as the workhorse of bioseparations $[26,27]$. Its relative high popularity is due to its simplicity as well as the wide range of adsorbents that are available. Bioseparation technologies based on adsorption are used for the removal of byproducts or the concentration of products (solute-solvent and solute-solute separations) [20]. The technologies used for these adsorption processes are fixed bed adsorption, expanded bed adsorption, simulated moving bed adsorption, and liquid chromatography [28]. Furthermore 
adsorption technology is emerging in relatively new hybrid technologies such as membrane adsorption, membrane chromatography and monolith chromatography [20, 23, 26, 27, 29].

Adsorption is the process of concentrating molecules on the surface of the adsorbent. It typically consist of four steps Figure 3: In the loading step the feed solution passes the adsorbent, adsorption of the target solute occurs, the feed solution is withdrawn in the washing step and last the target adsorbed solute is removed from the adsorbent. This last step is omitted if the adsorbent is discarded instead of regenerated.

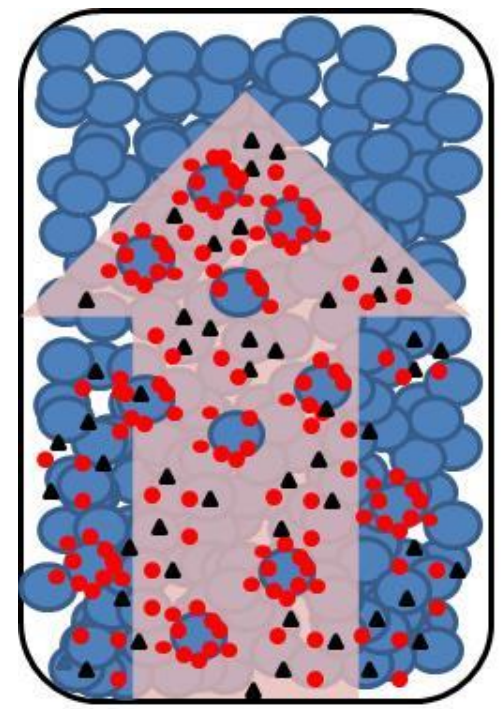

Load

Target solutes are adsorbed

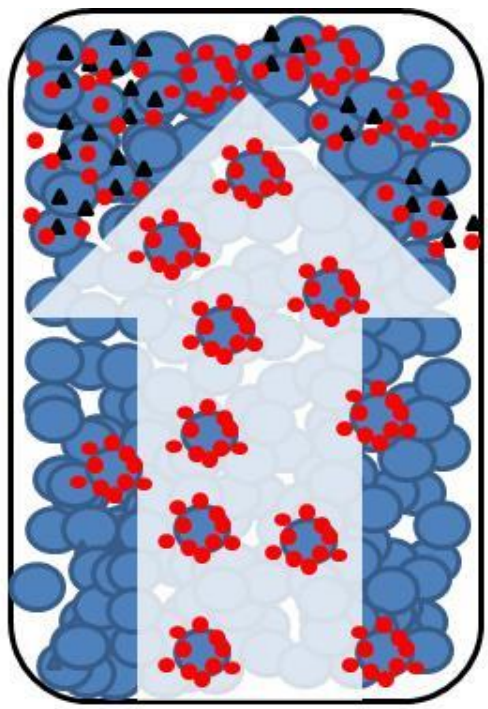

Wash

Unadsorbed solutes are washed away

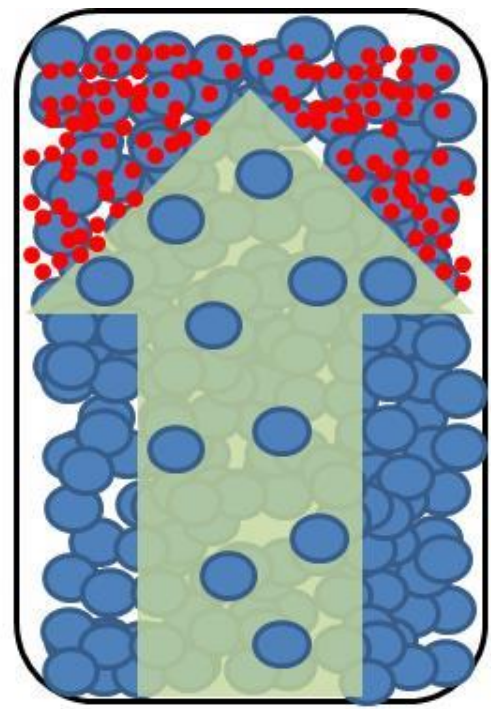

Elute

Target solutes are desorbed

Figure 3: Schematic representation of adsorption technology.

The selectivity of solutes for adsorbents is accomplished in different ways:

1. Thermodynamic equilibrium: different solute-adsorbent interaction lead to different adsorption equilibria. Typical bond energies are given in Table 1. The strength of the interaction is based on the bond energy as well as the number of bonds.

2. Kinetic effect: Differences in diffusion coefficient into the adsorbent. Kinetic separations are usually achieved with molecular sieve adsorbents with very specific pore openings such as zeolites and activated carbon. 
3. Size exclusion: The pore openings of the adsorbent are too small for pore diffusion into the adsorbent.

Table 1: Bond energies of common interactions for molecular imprinting.

Type of interaction

Van der Waals

Dipole-dipole

Charge-dipole

Hydrogen bond

Charge-Charge

\section{Approximate bond energy}

$0.1-1 \mathrm{~kJ} / \mathrm{mol}$

$\sim 1 \mathrm{~kJ} / \mathrm{mol}$

Up to $8 \mathrm{~kJ} / \mathrm{mol}$

Up to $40 \mathrm{~kJ} / \mathrm{mol}$

Up to $60 \mathrm{~kJ} / \mathrm{mol}$

\subsection{Adsorbents}

In industry a wide variety of adsorbents are used for bioseparations, they are usually based on carbons or synthetic resins [21]. Adsorbents are selected based on selectivity, capacity, regenerability, kinetics, compatibility and costs [28]. These properties are determined by material choice (e.g. polar or apolar matrix, functional groups), internal surface area and pore size distribution.

\subsubsection{Activated carbon}

Activated carbons are the eldest known adsorbents as centuries ago they were already used to remove tastes, odors and colors from water. Activated carbons are made by thermal decomposition from carbon containing materials such as wood, rice hulls, peat, lignin, coals, carbon black, nutshells etc. The carbonized materials are activated by a gas or chemical activation process. Activated carbons are produced with a full range of pore sizes. The surface of activated carbon is typical apolar and is therefore very suitable to remove apolar products from water streams. The main advantages of activated carbons are that they are cheap and available in many different pore sizes with high surface areas, unfortunately regeneration is often difficult. 


\subsubsection{Synthetic resins}

Synthetic resins are very suitable as adsorbents because the properties (physical and chemical) can be adapted to match the adsoption application. There are mainly three types of synthetic resins: gel-type, macroporous and hypercrosslinked resins [30]. Gel-type resins are solid single phase gel beads with low degrees of crosslinking. The adsorption is in the whole material and the capacity is therefore high. Unfortunately gel-type resins have low mechanical strength and are very susceptible for large volume change due to their low degree of crosslinking. However the swelling of the resins is necessary to make adsorption possible. Adsorption kinetics can be slow because diffusion is within the entire material.

Macroporous resins have higher degrees of crosslinking. They are manufactured in the presence of a porogen, after removal of the porogen a porous resin is obtained [28]. Adsorption is mostly at the surface of the pores and the capacity is therefore usually lower than in gel type resins. Due to the higher degree of crosslinking the resins are more stable than gel type resins.

Hypercrosslinked resins have superior adsorption characteristics, they are produced by postcrosslinking polymer chains or macroporous resins in solution or in swollen state [30-32]. This results in a highly crosslinked polymer network with low packing density and a network that is able to swell in polar and non-polar media due to the strongly strained state of the polymer when it is dry. The hypercrosslinked resins show higher internal surface areas and more uniform pore sizes with improved physical strength compared to macroporous resins and they are therefore superior to porous resins.

\subsubsection{Ion exchange resins}

Ion exchange resins are polymer resins with cat- anion exchange groups attached to them. In ion exchange adsorption the binding mechanism is an electrostatic interaction between the charged solute and the charged adsorbent [28]. The charged groups attached to the adsorbent (negative or positive) are neutralized by a mobile counter ion of opposite charge. When an ion from the solution is adsorbed at the charged adsorption site, it is exchanged with the ion that was already adsorbed to the adsorption site (Figure 4). 


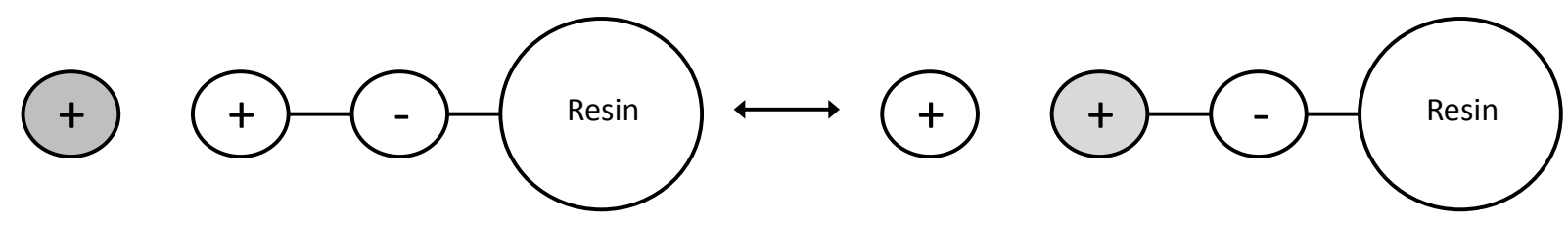

Figure 4: Principle of ion exchange.

Ion exchange usage is best known for water treatment. The first pharmaceutical application of ion exchange resins was in the 1950's when the antibiotic streptomycin was purified by Amberlite IRC50. Nowadays it is also used for bulk bioseparations such as for purification of microbial transglutaminase [33] lactic acid [34, 35] and whey proteins [36, 37]. Ion exchange resins are nowadays used for numerous bioseparations from the entire spectrum of bioproducts on a smaller scale [38].

\subsubsection{Affinity adsorption}

Affinity adsorption is the adsorption of a solute to a ligand attached to an adsorbent. Ligands form very selective bonds for solutes according to the lock-and-key principle [20]. The ligands can be substrate analogues, antigens, a specific base sequence, or a protein. Affinity ligands are divided into 2 groups: biological and synthetic ligands [39].

Biological ligands are derived from natural resources (e.g. DNA or RNA fragments, vitamins, coenzymes, antibodies). Biological ligands show high selectivity. However they are known to be more expensive and less stable to sterilization and cleaning, shortening their lifetime compared to synthetic ligands. Additionally, the purification methods sometimes uses human or animal derived products, this possesses a small risk of possibly dangerous contamination [24]. However, the main disadvantage is the low binding capacity of the biological ligands, increasing the capital cost of the process enormously.

Synthetic ligands are made by synthesis or by adaptation of existing molecular structures (e.g. pyrimidine's, non-natural peptides, etc.). Synthetic ligands are usually more simple and stable but are consequently less selective. Synthetic ligands are chosen based on either functional binding or structural binding. Knowledge on functional groups and/or shapes of the target functional groups or shape of the target molecule are used to bind with ligands. 


\subsubsection{Molecular imprinted polymers}

Although affinity separation shows the very high selectivity often required in bioseparations, the ligands are expensive, sensitive to denaturation and have a short lifespan, furthermore capacity of the ligand containing adsorbers is low. Recently researchers have tried to mimic the lock-and-key principle of affinity adsorbers with a cheaper and more stable alternative: molecular imprinting of polymers (MIP).

MIPs are polymers that contain functional groups, which are processed or polymerized in the presence of a template molecule (e.g. the targeted acid) (Figure 5) [40]. The polymer binding site, formed by these specific functional groups, is ordered in a three dimensional structure that makes the polymer also shape selective. The size and shape of the template molecule are mirrored in the size and shape of the binding site and the template fits exactly in the adsorption site of the polymer. A deviation of the molecular structure from the target molecule, such as an altered place of the molecules functional group, substitution of the templates functional group by another functional group or addition of a bulky group, hinders adsorption. This mechanism makes it possible to separate almost identical molecules from each other by adsorption with molecularly imprinted polymers.

Although in theory, due to their flexibility and the versatility in functional monomers, MIPs could potentially be very suitable for binding biological molecules. Most synthetic MIPs are built from highly cross-linked acrylate or vinyl polymers soluble in organic solvents and rebinding of biomolecules from an aqueous matrix is therefore not straightforward. Hydrophobic effects are usually strong in water and nonspecific adsorption of the template to the MIP surface is sometimes larger than the specific adsorption in the MIP cavities. Andersson et al [41] successfully imprinted the neuropeptide Leu-enkephalin and morphine and they were able to rebind the target molecules from an aqueous buffer, their research showed that the selectivity and the affinity in water were lower than in organic solvents. They found that the solvent used during imprinting should be as apolar as possible for aqueous applications. Later Andersson [42] imprinted the $\beta$-blocker (S)propranolol by carefully tuning $\mathrm{pH}$, ionic strength, buffer concentration and content of organic modifier and found that the target molecule could be rebound from various biological matrices, however addition of an organic solvent was necessary [43]. Haubt et al. synthesized MIPs for the 
herbicide 2,4-dichlorophenoxyacetic acid recognition using a mixture of water and methanol (4:1 v/v) [44]. Specifically designed functional monomers significantly improved molecular recognition ability in aqueous media of e.g. antibiotics [45] and ampicillin[46]. Furthermore a series of hydrophilic functional monomers, crosslinkers or co monomers have been used to reduce nonspecific adsorption and thereby improve molecular imprinting for aqueous applications (e.g. estradiol [47], bupivacaine[48]). Research has shown that MIPs that are selective for biomolecules from aqueous media need careful optimization in terms of functional monomer, cross linker, $\mathrm{pH}$, buffer, solvent etc. and often MIPs do not show selectivity in aqueous media at all [49]. And although some initial small steps towards MIPs for bioprocessing have been made, higher selectivity, lower demands of the adsorption matrix and better predictability MIP performance is necessary for MIPs to become viable for biotechnology applications.

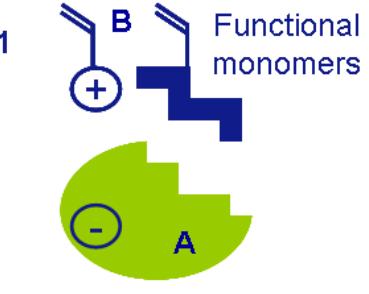

Target molecule

\section{Self assembly}

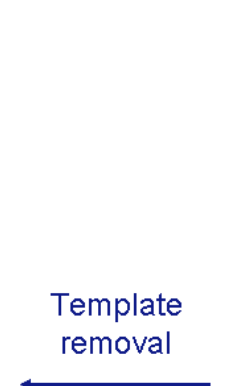

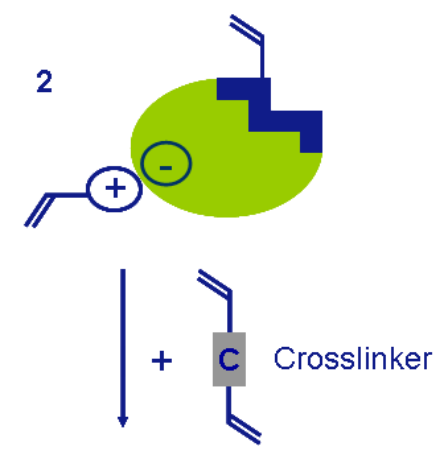

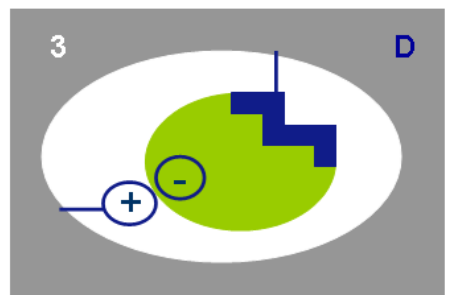

Figure 5: Illustration of the molecular imprinting process: 1: mixing of target molecule or template and reactants; 2 : selfassembling of target molecule and reactants; 3: stabilization of target molecule by crosslinking; 4: extraction of target molecule or template finally resulting in a polymer with shape and size selective binding site (MIP). 


\subsection{Adsorbent selection}

\subsubsection{Adsorption isotherms}

The adsorption capacity and selectivity of an adsorbent are mostly first studied with adsorption isotherms with batch experiments [28]. The isotherm is a plot of the solute adsorbed on the adsorbent as function of the concentration of the solution at equilibrium at constant temperature. The isotherm not only expresses the capacity of the adsorbent for a solute at a given equilibrium, the shape also gives information on the adsorption mechanism, surface properties and degree of affinity of the adsorbents [28]. There are many models that describe the adsorption of a solute on an adsorbent, some have a theoretical foundation, while others are only empirical. Often models are only valid in a small concentration range. Commonly used models to describe adsorption isotherms are Freundlich and Langmuir [28, 50].

The Langmuir adsorption isotherm is based on the assumption that an adsorbent can only be covered by one monolayer of adsorbate. When the adsorption sites are full a maximum adsorption capacity is reached [28].

$q_{e q}=\frac{b \cdot c_{e q} \cdot c_{\max }}{1+b \cdot c_{e q}}$

With $\mathrm{q}_{\mathrm{eq}}$ the adsorption capacity at equilibrium concentration $\mathrm{c}_{\mathrm{eq}}$, $\mathrm{b}$ the Langmuir equilibrium constant that indicates the energy of adsorption and $\mathrm{q}_{\max }$ the maximum adsorption capacity of the resin. At low concentrations this reduces to the linear correlation [28]:

$q_{e q}=b \cdot c_{e q} \cdot c_{\max }$

The Freundlich isotherm is an empirical isotherm that is often used for nonlinear isotherms at low concentrations [28].

$q_{e q}=K \cdot c_{e q}^{1 / n}$

With $\mathrm{q}_{\mathrm{eq}}$ the adsorption capacity at equilibrium concentration, $\mathrm{K}$ the Freundlich equilibrium constant that indicates the maximum adsorption capacity and $1 / \mathrm{n}$ the constant measuring the strength of adsorption. Although the Freundlich isotherm is an empirical expression, it can be derived from the 
theory that the heat of adsorption logarithmically decreases with increasing extend of adsorption [28].

\subsubsection{Zero length column experiments}

There are different experimental set-ups that can be used to determine adsorption kinetics of adsorbents. The zero length column (ZLC) introduced by Eic and Ruthven in 1988 is frequently used to study the adsorption kinetics of adsorbents [51] (Figure 6). In this experiment, a solute is pumped from a vessel over a very short column containing the adsorbent back to the vessel. The short bed length, the flow rate and the solution volume are chosen in such way that the concentration of the solution is almost equal in the entire set up at a given time, mimicking an ideal mixed finite bath. The concentration of the solution is measured in time. The time to reach the equilibrium adsorption concentration is a measure for the effectiveness of the adsorbent.

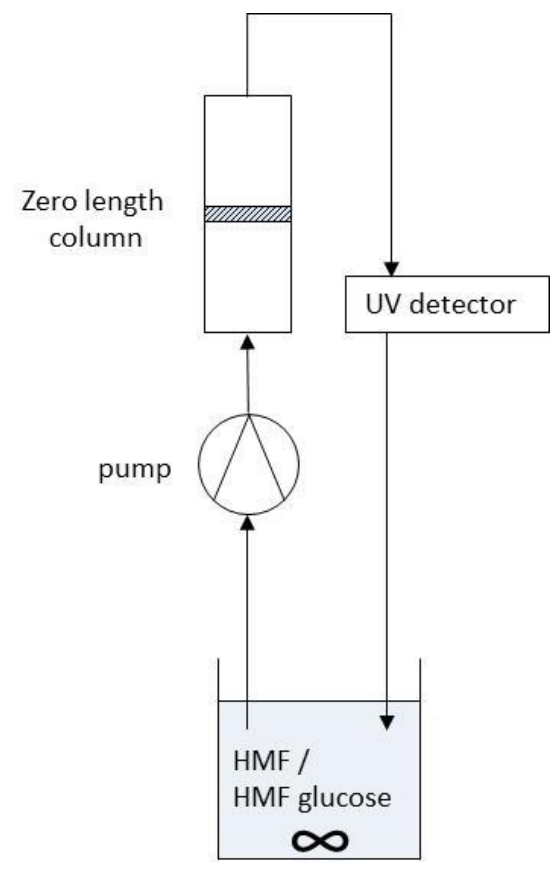

Figure 6: Schematic representation of experimental zero length column (ZLC) set up. 


\subsubsection{Breakthrough experiments}

Breakthrough curves are determined to examine properties of an adsorption column in continuous operation [21, 52]. A breakthrough curve is obtained by plotting the outlet concentration of the column versus the treated volume. From this curve the breakthrough point, column capacity and efficiency can be determined. The breakthrough point is defined as the point where the maximum allowed concentration solute is reached. The column capacity is the amount of solutes adsorbed at $100 \%$ loading of the column while the column efficiency compares this value with the value obtained from batch adsorption experiments. In contrast to equilibrium batch adsorption experiments, the kinetic properties of the adsorbents are also very important. The advantage of column experiments over batch experiments is that the adsorbent at the inlet is contacted continuously with the solution at equilibrium concentration and maximum loading can be achieved $[53,54]$.

Ideally the curve is sharp. In that case no solute comes out of the column until the adsorbent is saturated. The sharpness of the breakthrough curve is a measure of the adsorption kinetics, mass transfer and mixing in the flow system $[28,52]$.

\subsection{Alternative stationary phases}

Although a conventional packed bed is the most commonly used stationary phase in adsorption technology, it has some downsides. The pressure drop in the column is generally high, and it often increases during the process due to compaction of the adsorbent and accumulation of solids (such as colloidal material) $[26,29,55,56]$. Furthermore the mass transfer to the binding sites is largely dependent on the slow intra particle diffusion (Figure 7). Transport from the adsorption site to the recovery liquid is equally dependent on intra particle diffusion and the recovery liquid volume necessary is high. Packed beds are also susceptible to channeling, which is cause to the incomplete use of the column capacity. 


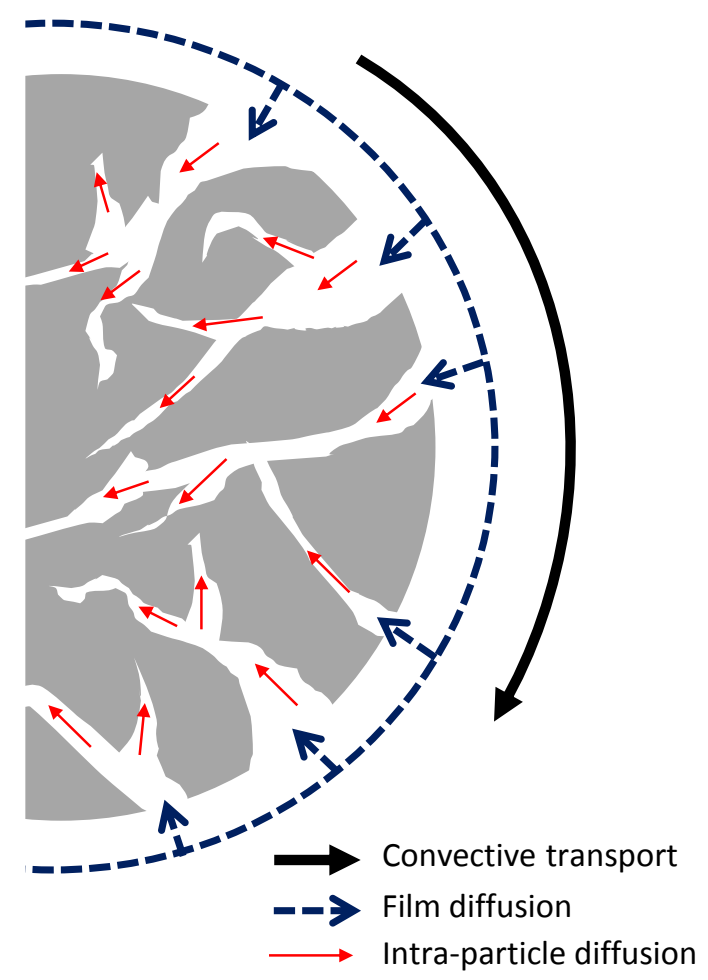

Figure 7: Solute transport in a porous adsorber adapted from [29]

The high costs involved in adsorption based bioseparations call for different type of adsorption stationary phases which make maximal use of convective transport, such as monoliths, membrane adsorbers or particle loaded mixed matrix membranes (Figure 8) [20, 26, 29]. These stationary phases consist of a very porous structure with interconnected pores that allow for uninterrupted bulk flow. The adsorption phase consists of the porous material itself $[57,58]$, the functional groups attached to it [59], an adsorption layer coated on top of it [60-62] or an adsorptive phase dispersed in the porous matrix (mixed matrix membrane) [63-68]. The biggest advantage is that the high porosity and short diffusion paths make very high flow rates possible. Compared to traditional packed beds, up to 100 times faster flow rates are sometimes realized without loss of adsorption efficiency. 


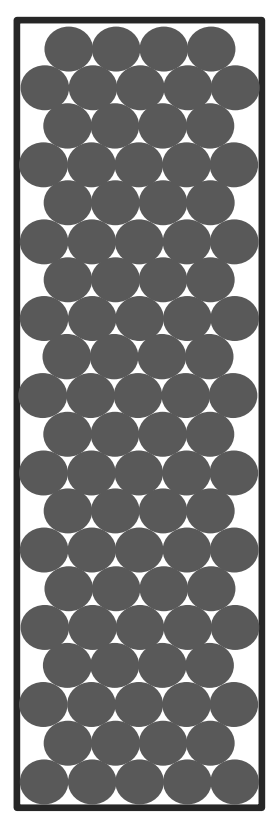

Packed bed

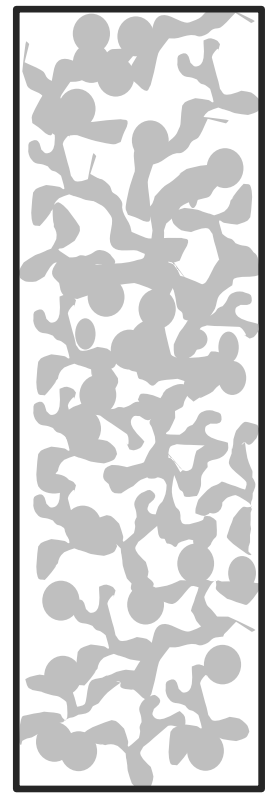

Monolith

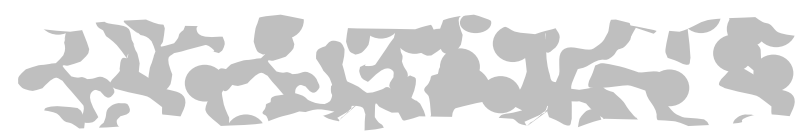

Membrane adsorber

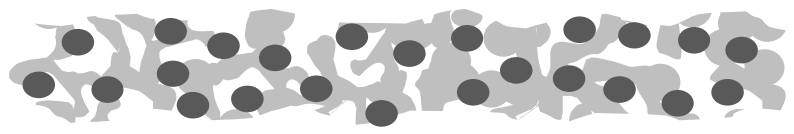

Particle loaded membrane adsorber (MMM)

Figure 8: Different stationary phases, adapted from [29].

Table 2: Comparison of chromatographic phases.

\begin{tabular}{|c|c|c|c|c|c|}
\hline Characteristic & & Packed bed & Monolith & $\begin{array}{l}\text { Membrane } \\
\text { adsorber }\end{array}$ & $\begin{array}{l}\text { Mixed matrix } \\
\text { membrane }\end{array}$ \\
\hline Flow rates & & Low & High & High & Intermediate \\
\hline Pressure drop & & High & Low-moderate & Low & Low \\
\hline $\begin{array}{l}\text { Dominant } \\
\text { transport }\end{array}$ & & Diffusion & Convection & Convection & $\begin{array}{l}\text { Convection- } \\
\text { Diffusion }\end{array}$ \\
\hline \multirow[t]{2}{*}{ Binding capacity } & $\begin{array}{l}\text { Small } \\
\text { molecules }\end{array}$ & High & Low & Low & Moderate-High \\
\hline & $\begin{array}{l}\text { Large } \\
\text { molecules }\end{array}$ & Low & High & High & Moderate \\
\hline Resolution & & High & Moderate & Moderate & Moderate-High \\
\hline
\end{tabular}




\subsubsection{Membrane adsorbers}

In membrane adsorbers (MA) the membranes function as a porous matrix for the adsorber phase and not as a separating phase itself. The advantage of MA's is that ideally most solute transport is by fast convective flow and slow diffusion is only over a short distance through the film diffusion layer (Figure 9A). In the case that adsorption kinetics itself are not the limiting factor, the adsorption time is therefore greatly reduced and the flow rate can be increased tremendously, compared to a conventional packed bed, without loss of capacity [20, 26, 29]. Furthermore the pore structure induces turbulent flow reducing the stagnant layer surrounding the adsorptive phase [4].

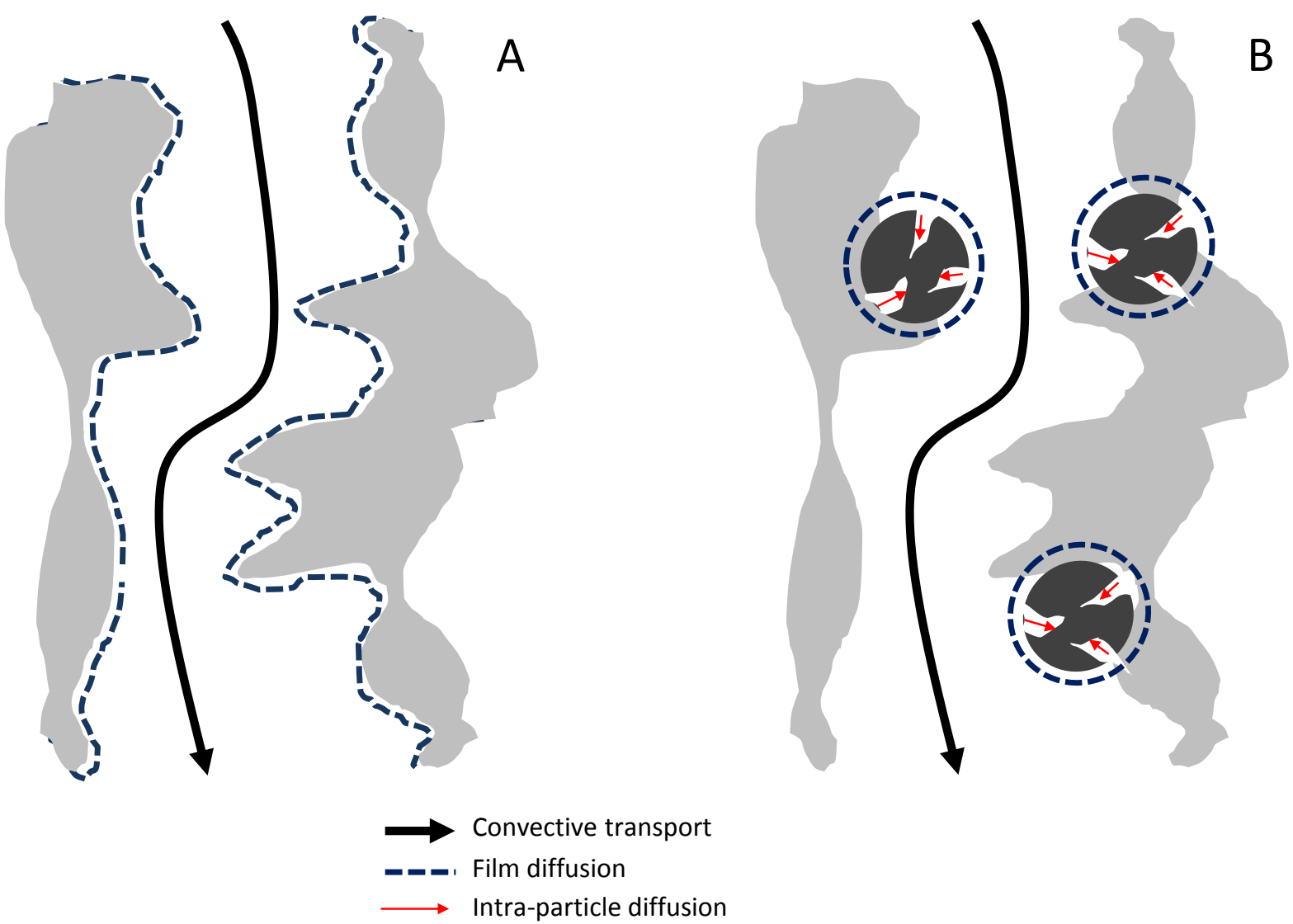

Figure 9: Solute transport in the pore of a membrane adsorber $(A)$ and the pore of a mixed matrix membrane (B).

Membrane chromatography with MA's is very suitable for large proteins. These large proteins normally do not enter the pores of conventional membrane adsorbers and only adsorb to the external surface area of the adsorption particles. The surface area of membranes is much larger than that of the adsorption particles and the adsorption capacity is therefore much higher. For smaller molecules, the adsorption capacity of MA's is lower than that of porous or gel type 
particles, but larger than that of nonporous rigid particles. The use of MAs is for small proteins is only advantageous when diffusion kinetics in conventional media are problematic.

Generally membrane adsorbers are prepared by applying an adsorption layer on a, mostly commercial, polymeric membrane, possessing the right chemical and physical properties [29]. Affinity ligands are the most common functionality of MA $[29,55]$. Other common functionalities are ion exchange and hydrophobic interactions.

The most important physical property of the support membrane is the pore size [29]. The pores should be large enough for the molecules to enter the pores. On the other hand, when the pores are too large for the application, the capacity decreases since the surface area is inversely related to the pore size. The pore size distribution and dead-end pores affect the flow distribution in the membrane and consequently lead to axial dispersion (peak broadening) [29]. The chemical properties of the membrane support affects the feasibility of modifying the membranes with functional groups. The chemical stability of the support is very important for the membrane to maintain stability in a wide range of $\mathrm{pH}$ 's, temperatures and ionic strengths endured during operation, sterilization and regeneration [29]. Furthermore the chemical properties of the support also determines the hydrophobicity of the membrane which is important for the non-specific adsorption and fouling properties of the membrane.

\subsubsection{Monoliths}

Monoliths are supports that consist of a single, continuous piece of a porous material. Most monoliths are produced by in-situ radical chain polymerization in the column [26, 56]. The presence of a porogen during the polymerization provides the monolith with interconnected pores when it is removed after the polymerization. The big advantage of monolith chromatographic processes is that, just like in MA, the transport of solutes to their binding sites predominantly takes place by convection (Figure 9). The capacity of monoliths is usually also lower than the capacity of porous particles. Only in the case of very large solutes that would only adsorb on the external surface of a particle, the capacity of the monoliths is higher due to the high surface to volume ratio of the porous stationary phase. 
Monoliths and membrane adsorbers differ from each other in the longitudinal dimension. Monoliths are comparable to a stack of membrane adsorbers. The nature of the polymerization method and the associated uniaxial pore formation due to the heat involved in polymerization, prevents a scale up for industrial applications and is the major cause to monoliths being used for small scale operations such as analytics. A solution to this problem is preparing tubular monoliths and merging them to one large monolith. Tubular monoliths can also be prepared by the very well investigated phase inversion method, often used in tubular hollow fiber membrane preparation.

\subsubsection{Mixed matrix membranes (MMM)}

The low capacity related to MA adsorbers and monoliths can be enhanced by using particle loaded mixed matrix membranes (MMM) $[63,68,69]$. Like in a conventional packed bed, the capacity is accomplished by the small porous adsorption phase which is dispersed in the membrane. Because the particles are very small, the diffusion length in the porous medium is much shorter than in conventional packed bed particles (Figure 9B) and adsorption is therefore much faster. The disadvantage of this technology compared to MA and monoliths is that the higher capacity is accompanied with slightly longer diffusion times due to intra particle diffusion. The flow through speed is therefore lower. The advantage of MMMs compared to conventional packed beds is that in that case small particles would lead to an enormous pressure drop in an conventional column, in the MMM this is prevented by the relative large pores of the membrane preventing close packing of the small particles.

\subsubsection{Membrane adsorber operating systems}

Generally three types of membrane adsorber operating systems are used: Flat sheet, hollow fiber, and radial flow (Figure 10) $[26,29]$. Flat sheet membrane adsorbers are often used in a stack of membranes. This increases the membrane volume but also ensures a simultaneous introduction of the liquid into the membranes. Furthermore imperfections of the membranes are leveled out. Flat sheet membranes are used in dead-end filtration mode. The disadvantage of this technique is that trapped particles on the surface easily build a cake layer on the surface. Hollow fiber membranes are often used in filtration application due to their large surface to volume ratio. They consist of hollow tubes typically ranging from 0.25 to $2.5 \mathrm{~mm}$. The tubes are bundled to provide membrane 
volume. The main advantage is their surface to volume ratio. They are however less suitable for membrane chromatography (especially pulse chromatography) since due to the cross flow the liquid enters the membrane pores at different times thereby enhancing peak broadening. On the other hand cross flow filtration however does help to prevent buildup of a cake layer of trapped particles that brings down the performance of the membrane. Radial flow adsorbers are prepared by winding a flat sheet membrane over a porous cylindrical core. Even greater flow distributions are expected since the liquid also enters the pores at different times and due to the enhanced membrane area in radial direction, the flow velocity is also reduced in the outward direction of the core. This makes the membrane unsuitable for pulse chromatography.

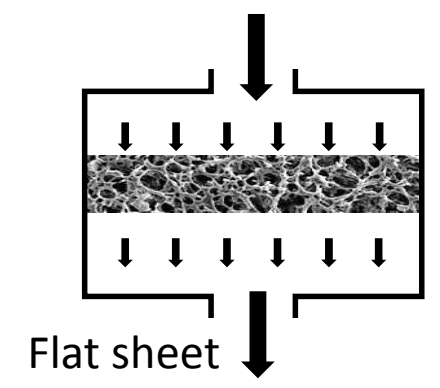

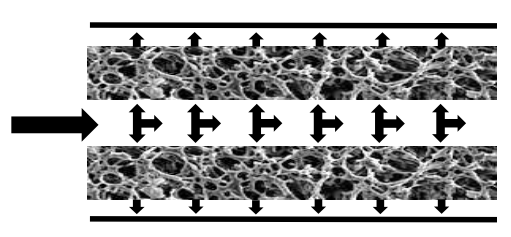

Hollow fiber

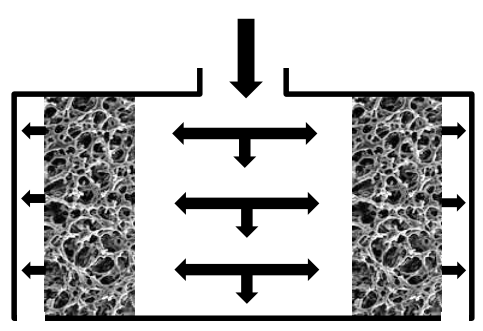

Radial flow

Figure 10: Common membrane adsorber operating types with their flow patterns.

Membrane adsorption technology is used more and more in bioseparations. A recent survey investigating the alternative downstream bioseparation technologies of biopharmaceutical manufacturers revealed an increased investment of these companies in membrane chromatography and monolith installations [23]. This shows that these technologies are already proving themselves in the biotechnology markets.

\subsection{Goal of this research}

The downstream processing of biomolecules often makes use of packed bed adsorption. The aim of this study is to improve this downstream processing by gaining better knowledge on the separation principles involved, using better adsorbents, improving adsorption kinetics, by using other stationary phases and by improving the adsorption selectivity. For this, we make use of: 
1. Selecting the best adsorbent, based on selectivity, capacity and adsorption kinetics.

2. Improving adsorption performance of adsorbents by incorporation of small adsorbents in an alternative stationary phase: a particle loaded mixed matrix membrane.

3. Improving the selectivity of the adsorbent by molecular imprinting.

\subsection{References}

1. Massoudi, A. and M. Mackenzie, US biotech stocks soar to new record high, in ft.com Februari 12, 2014: New York.

2. Hay, T., Biotechnology boom is here to stay, in Wall Street Journal DigitalOctober 14, 2013.

3. Alter, D., How to invest in biotech now and double your money. Money Morning, June 19, 2013.

4. Handbook of Bioseparations, ed. S. Ahuja. 2000, San Diego: Academic Press.

5. Harrison, R.G., et al., Bioseparation Science and Engineering, ed. K.E. Gubbins. 2003, New York: Oxford University Press.

6. Huang, H.J., et al., A review of separation technologies in current and future biorefineries. Separation and Purification Technology, 2008. 62(1): p. 1-21.

7. Hughes, B. and L.E. Hann, The production of biopharmaceuticals, in Biologics in General Medicine. 2007, Springer. p. 59-66.

8. Haro, P., et al., Potential routes for thermochemical biorefineries. Biofuels, Bioproducts and Biorefining, 2013. 7(5): p. 551-572.

9. Cheng, M.C., et al., Breeding an amylolytic yeast strain for alcoholic beverage production. Applied Biochemistry and Biotechnology, 2011. 163(6): p. 693-706.

10. Rasmussen, H., H.R. Sorensen, and A.S. Meyer, Formation of degradation compounds from lignocellulosic biomass in the biorefinery: sugar reaction mechanisms. Carbohydrate Research, 2014. 385(0): p. 45-57.

11. Abdel-Rahman, M.A., Y. Tashiro, and K. Sonomoto, Recent advances in lactic acid production by microbial fermentation processes. Biotechnology Advances, 2013. 31(6): p. 877-902.

12. Francavilla, M., et al., The red seaweed gracilaria gracilis as multi products source. Marine Drugs, 2013. 11(10): p. 3754-3776.

13. Nath, A., et al., Production, purification, characterization, immobilization, and application of b-galactosidase: A review. Asia-Pacific Journal of Chemical Engineering, 2014. in print.

14. Karg, S.R. and P.T. Kallio, The production of biopharmaceuticles in plant systems. Biotechnology Advances, 2009. 27(6): p. 879-894.

15. Kaur, V., et al., Welan Gum: Microbial production, characterization, and applications. International Journal of Biological Macrolecules, 2014. 65: p. 454-461.

16. Patel, S., Rose hip as complementary and alternative medicine: Overview of the present status and prospects. Mediterranean Journal of Nutricion and Metabolism, 2013. 6(2): p. 89-97.

17. Farid, M.A., et al., Statistical optimization of glucose oxidase production from Aspergillus niger NRC9 under submerged fermentation using response surface methodology. Annals of Microbiology, 2013. 63(2): p. 523-531.

18. Rosas-Garcia, N.M., Biopesticide production from Bacillus thuringiensis: An environmentally friendly alternative. Recent Patents on Biotechnology, 2009. 3(1): p. 2836.

19. Market Watch, NASDAQ Biotechnology Index. [cited 2014 May 1].

20. Ghosh, R., Principles of bioseparations engineering. 2006, Singapore: World Scientific Publishing Co. Pte. Ltd.

21. Belter, P.A., E.L. Cussler, and W.-s. Hu, Bioseparations, downstream processing for biotechnology. 1988, New York: John Wiley \& Sons. 
22. Lightfood, E.N., Speeding the design of bioseparations: $A$ heuristic aproach to engineering design. Industrial and Engineering Chemistry Research, 1999. 38(10): p. 3628-3634.

23. Tran, R., et al., Changing manufacturing paradigms in downstream processing and the role of alternative bioseparation technologies. Journal of Chemical Technology \& Biotechnology, 2013.

24. Low, D., R. O'Leary, and N.S. Pujar, Future of antibody purification. Journal of Chromatography B: Analytical Technologies in the Biomedical and Life Sciences, 2007. 848(1): p. 48-63.

25. Dwyer, J.L., Economics of separation processes. Biotechnology, 1984. 2: p. 957.

26. Ghosh, R., Protein separation using membrane chromatography: Oportunities and challenges. Journal of Chromatography A, 2002. 952(1-2): p. 13-27.

27. Przybycien, T.M., N.S. Pujar, and L.M. Steele, Alternative bioseparations operations: life beyond packed-bed chromatography. Current Opinion in Biotechnology, 2004. 15(5): p. 469-478.

28. Thomas, W.J. and B. Crittenden, Adsorption Technology and Design. 1998: Butterworth Heinemann.

29. Orr, V., et al., Recent advances in bioprocessing application of membrane chromatography. Biotechnology Journal, 2013. 31(4): p. 450-465.

30. Xu, Z., Q. Zhang, and H.H.P. Fang, Applications of Porous Resin Sorbents in Industrial Wastewater Treatment and Resource Recovery. Critical Reviews in Environmental Science and Technology, 2003. 33(4): p. 363-389.

31. Tsyurupa, M.P. and V.A. Davankov, Porous stucture of hypercrosslinked polystyrene: State-of-the-art mini-review. Reactive and Functional Polymers, 2006. 66(7): p. 678-779.

32. Jerabek, K., L. Hankova, and Z. Prokop, Post-crosslinked polymer adsorbents and their properties for separation of furfural from aqueous solutions. Reactive Polymers, 1994. 23(2-3): p. 107-112.

33. Zhu, Y., et al., Microbial transglutaminase - a review of its production and application in food processing. Applied Microbiology and Biotechnology, 1995. 44(3-4): p. 277-282.

34. Ataei, S.A. and E. Vasheghani-Farahani, In situ separation of lactic acid from fermentation broth using ion exchange resins. Journal of Industrial Microbiology \& Biotechnology, 2008. 35(11): p. 1229-1233.

35. Tong, W.-Y., et al., Purification of I(+)-lactic acid from fermentation broth with paper sludge as a cellulosic feedstock using weak anion exchanger Amberlite IRA-92. Biochemical Engineering Journal, 2004. 18(2): p. 89-96.

36. Doultani, S., K.N. Turhan, and M.R. Etzel, Fractionation of proteins from whey using cation exchange chromatography. Process Biochemistry, 2004. 39(11): p. 1737-1743.

37. Tek, H.N., K.N. Turhan, and M.R. Etzel, Effect of Conductivity, $\mathrm{pH}$, and Elution Buffer Salinity on Glycomacropeptide Recovery from Whey Using Anion Exchange Chromatography. Journal of Food Science, 2005. 70(4): p. E295-E300.

38. Kammerer, J., R. Carle, and D.R. Kammerer, Adsorption and Ion Exchange: Basic Principles and Their Application in Food Processing. Journal of Agricultural and Food Chemistry, 2011. 59(1): p. 22-42.

39. Clonis, Y.D., Affinity chromatography matures as bioinformatic and combinatorial tools develop. Journal of Chromatography A, 2006. 1101(1-2): p. 1-24.

40. Yan, J., X. Wang, and J. Chen, Swelling of porous styrene-divinylbenzene copolymers in water. Journal of Applied Polymer Science, 2000. 75(4): p. 536-544.

41. Andersson, L.I., et al., Mimics of the binding sites of opioid receptors obtained by molecular imprinting of enkephalin and morphine. Proceedings of the National Academy of Sciences of the United States of America, 1995. 92(11): p. 4788-4792.

42. Andersson, L.I., Application of molecular imprinting to the development of aqueous buffer and organic solvent based radioligand binding assays for (S)-propranolol. Analytical Chemistry, 1996. 68(1): p. 111-117.

43. Bengtsson, H., U. Roos, and L. I. Andersson, Molecular Imprint Based Radioassay for Direct Determination of S-Propranolol in Human Plasma. Analytical Communications, 1997. 34(9): p. 233-235.

44. Haupt, K., A. Dzgoev, and K. Mosbach, Assay system for the herbicide 2,4dichlorophenoxyacetic acid using a molecularly imprinted polymer as an artificial recognition element. Analytical Chemistry, 1998. 70(3): p. 628-631. 
45. Urraca, J.L., et al., A stoichiometric molecularly imprinted polymer for the class-selective recognition of antibiotics in aqueous media. Angewandte Chemie - International Edition, 2006. 45(31): p. 5158-5161.

46. Lubke, C., et al., Imprinted polymers prepared with stoichiometric template-monomer complexes: efficient binding of ampicillin from aqueous solutions. Macromolecules, 2000. 33(14): p. 5098-5105.

47. Meng, Z., W. Chen, and A. Mulchandani, Removal of estrogenic pollutants from contaminated water using molecularly imprinted polymers. Environmental Science and Technology, 2005. 39(22): p. 8958-8962.

48. Dirion, B., et al., Water-Compatible Molecularly Imprinted Polymers Obtained via HighThroughput Synthesis and Experimental Design. Journal of the American Chemical Society, 2003. 125(49): p. 15101-15109.

49. Zhang, H., Water-compatible molecularly imprinted polymers: Promising synthetic substitutes for biological receptors. Polymer, 2014. 55(3): p. 699-714.

50. Masel, R.I., Principles of Adsorption and Reaction on Solid Surfaces. 1996, United states of America: Wiley.

51. Eic, M. and D.M. Ruthven, $A$ new experimental technique for measurement of intracrystalline diffusivity. Zeolites, 1988. 8(1): p. 40-45.

52. Hand, D.W., J.C. Crittenden, and W.E. Thacker, Simplified models for design of fixed-bed adsorption systems. Journal of Environmental Engineering-Asce, 1984. 110(2): p. 440-456.

53. Ahmad, A.A. and B.H. Hameed, Fixed-bed adsorption of reactive axo dye onto granular activated carbon prepared from waste. Journal of Hazardous Materials, 2010. 175(1-3): p. 298-303.

54. Banat, F., et al., Bench-scale and packed bed sorption of methylene blue using treated olive pomace and charcoal. Bioresource Technology, 2007. 98(16): p. 3017-3025.

55. Avramescu, M.-E., Z. Borneman, and M. Wessling, Membrane chromatography, in Handbook of Membrane Separations; Chemical, Pharmaceutical, Food and Biotechnological Applications, A.K. Pabby, S.S.H. Rizvi, and A.M. Sastre, Editors. 2008, CRC Press.

56. Liu, K., et al., Organic monoliths for high-performance reversed-phase liquid chromatography. J Sep Sci, 2013. 36(17): p. 2767-81.

57. Tennikova, T.B. and F. Svec, High-performance membrane chromatography: Highly efficient separation method for proteins in ion-exchange, hydrophobic interaction and reversed-phase modes. Journal of Chromatography A, 1993. 646(2): p. 279-288.

58. Hjertén, S., J.-L. Liao, and R. Zhang, High-performance liquid chromatography on continuous polymer beds. Journal of Chromatography A, 1989. 473(0): p. 273-275.

59. Selvakumar, R., et al., Effect of Biotin and Galactose Functionalized Gelatin Nanofiber Membrane on HEp-2 Cell Attachment and Cytotoxicity. The Journal of Membrane Biology, 2014. 247(1): p. 35-43.

60. Piletsky, S.A., et al., Surface functionalization of porous polypropylene membranes with molecularly imprinted polymers by photograft copolymerization in water. Macromolecules, 2000. 33(8): p. 3092-3098.

61. Schneider, F., et al., Comparison of thin-layer and bulk MIPs synthesized by photoinitiated in situ crosslinking polymerization from the same reaction mixtures. Journal of Applied Polymer Science, 2005. 98(1): p. 362-372.

62. Raiado-Pereira, L., et al., Grafting hydrophobic and affinity interaction ligands on membrane adsorbers: A close-up "view" by X-ray photoelectron spectroscopy. Separation and Purification Technology, 2012. 93(0): p. 75-82.

63. Avramescu, M.-E., Z. Borneman, and M. Wessling, Dynamic behavior of adsorber membranes for protein recovery. Biotechnology and Bioengineering, 2003. 84(5): p. 564572.

64. Saiful, Z. Borneman, and M. Wessling, Enzyme capturing and concentration with mixed matrix membrane adsorbers. Journal of Membrane Science, 2006. 280(1-2): p. 406-417.

65. Tijink, M.S.L., et al., A novel aproach for blood purification: Mixed matrix membranes combining diffusion and adsorption in one step. Acta Bomaterialia, 2012. 8(6): p. 22792287.

66. Tetala, K.K.R., et al., A metal ion charged ixed matrix membrane for selective adsorption of hemoglobin. Separation and Purification Technology, 2013. 115(0): p. 20-26. 
67. Saufi, S.M. and C.J. Fee, Fractionation of b-Lactoglobulin from whey by mixed matrix membrane ion exchange chromatography. Biotechnology and Bioengineering, 2009. 103(1): p. 138-147.

68. Avramescu, M.-E., Z. Borneman, and M. Wessling, Mixed-matrix membrane adsorbers for protein separation. Journal of Chromatography A, 2003. 1006(1-2): p. 171-183.

69. Avramescu, M.-E., Z. Borneman, and M. Wessling, Particle-loaded hollow-fiber membrane adsorbers for lysozyme separation. Journal of Membrane Science, 2008. 322(2): p. 306313. 


\section{Performance analysis of aromatic adsorptive resins}

\section{for the effective removal of furan derivatives from}

\section{glucose}

Journal of Chemical Technology and Biotechnology, 2014. 90(1): p. 101-109

\subsection{Abstract}

Batch adsorption experiments were carried out to evaluate the removal of 5-hydroxymethylfurfural (HMF) from glucose for further processing by fermentation. In contrast to previous research, though essential to understand the mechanism, it investigates resins with a similar matrix while the functional group is systematically varied. The experiments were carried out with 5 different styrene based resins (with and without anion exchange groups) with the aim to find a relation between chemical and physical material properties and HMF and glucose adsorption. The experiments showed that a high surface area is a key property for effective HMF adsorption. Although introduction of polar groups in the form of anion exchange groups appears to increase the affinity of the resin material for HMF, these groups also introduce affinity for glucose, resulting in loss of fermentable sugar. Competitive adsorption studies of HMF and glucose showed that glucose does not affect HMF adsorption in any of the resins. Dowex Optipore L-493 shows a high specific HMF adsorption and no specificity for glucose, which makes it an excellent adsorber for HMF removal from hydrolysate for the fermentation of glucose.

Keywords: aromatic resin, adsorption, HMF, sugars, biorefinery.

\subsection{Introduction}

Many countries have set goals to replace conventional energy sources with renewable energy sources, because of growing energy demands, greenhouse emissions and depleting oil and natural gas resources [1, 2]. This has led to investigations into the use of biomass as a feedstock for 
renewable fuels and base chemicals [3]. Biofuels derived from lignocellulosic biomass could fulfill a large demand of this urge for biobased fuels responsibly [4]. Lignocellulosic biomass not only includes dedicated energy crops but also agricultural residues (corn stover and sugarcane bagasse), wood residues and municipal paper waste [5]. Lignocellulosic biomass is biomass of plants and consists of cellulose, hemicellulose and lignin. Cellulose and hemicellulose are carbohydrate polymers that, once removed from the lignin, can be hydrolysed into sugars (glucose and xylose). After removal of the impurities induced by the hydrolysation, these sugars can serve as a feedstock to produce ethanol, lactic acid and other valuable chemicals.

Sulfuric acid is a well know catalyst for the hydrolysation of cellulose and hemicellulose in biomass to sugars [5]. Unfortunately this can lead to formation of byproducts (acetic acid) and to degradation of lignin to phenolic compounds and of the sugars to furfural (from xylose) and 5hydroxymethylfurfural (HMF) (from glucose) [4]. These components are toxic for the bacteria in the downstream fermentation process and need to be removed before further processing. Several authors showed that these toxins can be removed by adsorption. Polymeric adsorbers [6-9], catand anion exchange resins (weak and strong) [10-13], zeolites [2] and activated carbon [13-15] have shown to remove toxins. Because of regeneration, stability and capacity, polymeric adsorbers (resins), with and without ion exchange groups, are considered most promising for adsorption of these toxins $[5,16]$.

Acids and phenolic compounds adsorb best on anion exchange resins [17]. However, research on furan adsorption is inconclusive on the best adsorption resin for furan adsorption. Dowex recommends the use of a styrene-divinylbenzene (DVB) based weak base ion exchange resin (Optipore SD-2) for the removal of the furan 5-hydroxylethylfurfural (HMF) [18]. While literature has shown good results for HMF removal with anion exchange resins [1], it is probably the non polar styrene-DVB matrix that is responsible for furan adsorption $[6,16]$. Some authors have compared the adsorption of furans on weak anion exchange, strong anion exchange resins and polymeric adsorbers $[10,17]$. Unfortunately the matrix of the different resins varied (acrylic or styrene based) making it difficult to relate the adsorption performance purely to the presence or absence of ion exchange groups. Fargues et al. [16] made a thorough comparison based on single component isotherms and breakthrough curves of ion exchange (weak and strong) resins with polymeric 
adsorbers, all with styrene-DVB based matrices. Although the good furan adsorption on the styrene-DVB resin is consistent with the findings of others $[6,7,17]$, the low adsorption of furans on styrene based anion exchange resins is not consistent with the findings of others [10, 12, 17]. Other research on this subject only examines the effect of adding different resins to the hydrolysate on the effectiveness of the fermentation $[9,10,12]$. Although this leads to a selection of resins that improve fermentation production, it does not provide further insight into the adsorption mechanisms involved (e.g. specificity of adsorption of toxin, competitive adsorption, sugar loss) and therefore design of specific resins for this rapidly increasing industry is difficult. Although literature has shown that a styrene based resin is a good furan adsorber, the addition of polar anion exchange groups in the resin on the furan adsorption is not well understood.

This study aims to provide more insight in the adsorption mechanism of furans for the removal of furans from sugar on commercial styrene-DVB based resins. In contrast to previous research [1], this study investigates resins with a similar matrix. Two styrene based resins and three different styrene based anion exchange resins are investigated. Insight in the adsorption mechanism is obtained by relating the adsorption isotherms of HMF and glucose (pure and competitive) to the physical (BET surface area, pore size, pore volume, swelling and water uptake) and chemical properties (none, weak or strong anion exchange groups) of the resins. HMF and glucose are chosen as model components. HMF represents a large class of furan toxins and is therefore chosen as a model component for furans, while glucose represents a large class of sugars and is therefore chosen as model component for sugar (see Table 1). 
Table 1: Physiochemical properties of solutes.

\begin{tabular}{lllll}
\hline Solute & Mw (g/mol) & Log $\mathrm{K}_{\text {ow }}$ & pKa & \\
\hline $\mathrm{HMF}$ [19] & 126.11 & -0.09 & & \\
Glucose [20, 21] & 180.16 & -2.82 & & \\
\hline
\end{tabular}

\subsection{Materials and Methods}

\subsubsection{Materials}

The resins Amberlite XAD4 (XAD4), Dowex Optipore L-493 (Optipore), Lewatit MP 62 (MP62), Lewatit M500 (M500) and Reillex HP (PVP) were purchased from Sigma Aldrich (Table 2). All resins have a styrene matrix crosslinked with divinylbenzene (DVB). The matrix of all resins therefore fully consists of polar aromatic rings. The resins MP62, M500, and PVP contain the following alkaline functional groups attached to the matrix: tertiary amine, 4-vinylpyridine and quaternary amine respectively. All resins are macroporous, except for Lewatit M500, which is a gel type resin. 5-hydroxymethylfurfural (HMF), glucose, sulfuric acid $\left(\mathrm{H}_{2} \mathrm{SO}_{4}\right)$ and sodium hydroxide $(\mathrm{NaOH})$ were obtained from Sigma Aldrich. 


\begin{tabular}{|c|c|c|c|c|c|c|c|}
\hline Resin & Matrix & Type & & $\begin{array}{l}\text { Functional } \\
\text { group }\end{array}$ & $\begin{array}{l}\text { Ion exchange } \\
\text { capacity (min. } \\
\text { eq/l) }\end{array}$ & $\begin{array}{l}\text { Surface } \\
\left(\mathrm{m}^{2} / \mathrm{g}\right)\end{array}$ & area \\
\hline XAD4 [22] & $\begin{array}{l}\text { Poly(styrene- } \\
\text { DVB) }\end{array}$ & Adsorbent & & None & - & $>750$ & \\
\hline Optipore [23] & $\begin{array}{l}\text { Poly(styrene- } \\
\text { DVB }^{\star} \text { ) }\end{array}$ & Adsorbent & & None & - & $>1100$ & \\
\hline MP62 [24] & $\begin{array}{l}\text { Poly(styrene- } \\
\text { DVB) }\end{array}$ & $\begin{array}{l}\text { Weak } \\
\text { anion } \\
\text { exchanger }\end{array}$ & base & Tertiary amine & 1.7 & n.a. & \\
\hline PVP [25] & $\begin{array}{l}\text { Poly(styrene- } \\
\text { DVB) }\end{array}$ & $\begin{array}{l}\text { Weak } \\
\text { anion } \\
\text { exchanger }\end{array}$ & base & Vinyl pyridine & 1.7 & n.a. & \\
\hline M500 (Cl') [26] & $\begin{array}{l}\text { Poly(styrene- } \\
\text { DVB) }\end{array}$ & $\begin{array}{l}\text { Strong } \\
\text { anion } \\
\text { exchanger }\end{array}$ & base & $\begin{array}{l}\text { Quaternary } \\
\text { amine }\end{array}$ & 1.3 & n.a. & \\
\hline
\end{tabular}

${ }^{*}$ Crosslinker not specified by Dow, crosslinker obtained from Ramaswamy et al. [27] and Vern et al. [9]

XAD4 and Optipore are polymeric adsorbents without functional groups. They have the same chemical structure, a styrene based matrix crosslinked with DVB, but they have different physical properties (e.g. surface area and pore size). They are selected to determine the HMF and glucose adsorption capacity of the styrene-DVB matrix. Because they have different surface areas, the adsorption capacity can also be related to the surface area. HMF is more apolar than glucose and it is therefore expected that it will adsorb on the apolar styrene resin while the polar glucose is less or not adsorbed. This is also seen by the relative lower log octanol/water partition coefficient (log $\mathrm{K}_{\mathrm{ow}}$ ) of glucose compared to $\mathrm{HMF}$, which represents the solubility in octanol relative to the solubility in water (Table 3). MP62 and PVP are weak base anion exchange resins and M500 is a strong base anion exchange resin. These resins have the same matrix as XAD4 and Optipore, but with 
different alkaline functional groups (tertiary amine, vinylpyridine and quartinary amine respectively). These polar alkaline groups can alter the affinity for HMF and/or glucose of the adsorbent, as well as the swelling in water of the resin due to the introduction of the polar groups.

To remove impurities present in the resins, all resins were rinsed with MilliQ water prior to use until no impurities were visible by UV-Vis $(200-400 \mathrm{~nm}) . \mathrm{M} 500$ is received in the $\mathrm{Cl}^{-}$form $\left(\mathrm{M} 500\left(\mathrm{Cl}^{-}\right)\right)$ and is also brought into the $\mathrm{OH}^{-}$form $\left(\mathrm{M} 500\left(\mathrm{OH}^{-}\right) .10 \mathrm{~g} \mathrm{M} 500\left(\mathrm{Cl}^{-}\right)\right.$was rinsed with a $500 \mathrm{ml} 1 \mathrm{M}$ sodium hydroxide $(\mathrm{NaOH})$ solution (24 hours). The solution was refreshed 4 times to ensure complete exchange of $\mathrm{Cl}^{-}$to $\mathrm{OH}^{-}$. The resins were rinsed with MilliQ water to remove excess $\mathrm{NaOH}$ (24 hours).

\subsubsection{Resin properties}

\section{Particle size}

The particle size distribution of all resins were measured by dynamic light scattering (DLS) at $25^{\circ} \mathrm{C}$ in a Malvern Zetasizer HS3000. A nominal $5 \mathrm{~mW}$ Helium Neon laser with a wavelength of $633 \mathrm{~nm}$ was used to measure the particle size distributions under angles of $12^{\circ}$ and $90^{\circ}$. The correlograms were analyzed using the CONTIN algorithm.

\section{Nitrogen adsorption}

The specific surface area (BET), pore size and pore volume were measured using nitrogen adsorption at $-195.8^{\circ} \mathrm{C}$ with a Micromeritics Tristar 3000 [28]. Before the measurement the resins were heated overnight to $80^{\circ} \mathrm{C}$ under nitrogen flow.

\section{Volume change and water uptake}

The volume change and water uptake of the resins under the influence of water was investigated. The volume of the resins was measured after the resins were equilibrated in water (24 hours). The resins were subsequently removed from the excess water by filtration and their mass was determined. The mass and volume were measured again after the resins were dried in a vacuum 
oven at $80^{\circ} \mathrm{C}$ (24 hours). This mass was used to determine the swelling and the water uptake with the following equations (Equation 1 and 2):

$$
S=\frac{V_{\text {wet }}-V_{\text {dry }}}{m_{\text {dry }}}
$$

With $S$ the volume change per gram dry resin $\left(\mathrm{ml} / \mathrm{g}_{\text {dry resin }}\right), \mathrm{V}_{\text {wet }}$ and $\mathrm{V}_{\text {dry }}$ the volume $\mathrm{ml}$ of the wet and dry resin respectively, and $m_{d r y}$ the mass of the dry resin $\left(g_{d r y}\right.$ resin $)$.

$$
W U=\frac{\left(m_{\text {wet }}-m_{\text {dry }}\right)}{m_{\text {dry }} \cdot \rho_{H 2 O}}
$$

With WU the water uptake per gram dry resin $\left(\mathrm{ml} / \mathrm{g}_{\text {dry resin }}\right), \mathrm{m}_{\text {wet }}$ and $\mathrm{m}_{\text {dry }}$ the mass $(\mathrm{g})$ of the wet and dry resin respectively and $\rho_{\mathrm{H} 2 \mathrm{O}}$ the density of water $(\mathrm{g} / \mathrm{ml})$.

\subsubsection{Adsorption isotherms}

HMF and glucose adsorption isotherms were determined to investigate the capacity, affinity and selectivity of the different resins (XAD4, Optipore, MP62, M500 $\left(\mathrm{CL}^{-}, \mathrm{OH}^{-}\right)$and PVP) for HMF and glucose. Adsorption behavior of the resins was studied by measuring the following adsorption isotherms:

1. Single HMF adsorption (low and high HMF concentrations)

2. Single glucose adsorption

3. Competitive HMF/glucose adsorption

4. HMF adsorption as function of temperature

The maximum adsorption capacity was determined from the HMF adsorption isotherms at high HMF concentrations in water (1000-20,000 mg/l). The HMF binding strength and adsorption capacity of the resins was investigated at low HMF concentrations $(1-30 \mathrm{mg} / \mathrm{l})$ in water since this gives more information on the affinity of the materials for HMF. Furthermore this is usually the concentration range of the targeted industrial processes. Glucose adsorption of the resins was investigated with glucose concentrations ranging from $1-100 \mathrm{~g} / \mathrm{l}$ in water. 
Competitive adsorption of HMF and glucose was studied by measuring HMF adsorption in a glucose solution (100 g/l). HMF adsorption from a glucose solution was determined at low (1-30 $\mathrm{mg} / \mathrm{l})$ concentrations for Optipore and XAD4 and due to analysis limitations, at high HMF concentrations (1000-20,000 mg/l) for the other resins. All these experiments were performed at 20 ${ }^{\circ} \mathrm{C}$.

Additionally the effect of temperature on the HMF adsorption capacity of Optipore was studied at 20 and $60{ }^{\circ} \mathrm{C}$ and $\mathrm{HMF}$ concentrations from $1000-20,000 \mathrm{mg} / \mathrm{l}$ in water.

In general $25 \mathrm{ml}$ of solution was added to $0.3 \mathrm{~g}$ wet resin in a $30 \mathrm{ml}$ flask. The solutions were equilibrated for 48 hours (MP 62) or 24 hours (all other resins) in a thermostatic shaking bath (20 ${ }^{\circ} \mathrm{C}$ or $60{ }^{\circ} \mathrm{C}$ ). The equilibration time for the solute adsorption was determined in separate experiments (not shown in this article). After the equilibration time, the equilibrium concentration was determined with HPLC.

HMF and glucose concentrations were determined with a Waters HPLC with a 515 pump, a 2487 dual $\lambda$ adsorbance detector and Millenium ${ }^{32}$ (version 3.20) software. Analysis was carried out using the column Resex ROA-organic acid $300 \times 7.8 \mathrm{~mm}$ (Phenomenex). The mobile phase was $0.005 \mathrm{M}$ $\mathrm{H}_{2} \mathrm{SO}_{4}$. The column was operated at $90{ }^{\circ} \mathrm{C}$ with a flow rate of $0.3 \mathrm{ml} / \mathrm{min}$ of the mobile phase. Peak detection was performed with UV at $284 \mathrm{~nm}$ (HMF) and $205 \mathrm{~nm}$ (glucose).

At high concentrations the adsorption isotherms are described by Langmuir sorption [29] (Equation 3):

$$
q=\frac{b \cdot c_{e q} \cdot q_{\max }}{1+b \cdot c_{e q}}
$$

With $\mathrm{q}\left(\mathrm{g}_{\text {solute }} / \mathrm{g}_{\text {resin }}\right)$ the adsorption capacity at equilibrium concentration $\mathrm{c}_{\text {eq }}\left(\mathrm{g}_{\text {solute }} / \mathrm{l}\right)$, b $\left(1 / \mathrm{g}_{\text {solute }}\right)$ the Langmuir equilibrium constant that indicates the energy of adsorption, which increases when the strength of the adsorption bond increases and $\mathrm{q}_{\max }\left(\mathrm{g}_{\text {solute }} / \mathrm{g}_{\text {resin }}\right)$ the maximum adsorption capacity of the resin.

At low concentrations the adsorption isotherms are correlated using Freundlich [29] (Equation 4): 
With $\mathrm{q}\left(\mathrm{mg}_{\text {solute }} / \mathrm{g}_{\text {resin }}\right)$ the adsorption capacity at equilibrium concentration $\mathrm{c}_{\text {eq }}\left(\mathrm{mg}_{\text {solute }} / \mathrm{l}\right), \mathrm{K}\left(\mathrm{l} / \mathrm{g}_{\text {resin }}\right)$ the freundlich equilibrium constant that indicates the maximum adsorption capacity and $1 / n$ the constant measuring the strength of adsorption. A lower $1 / n$ indicates a higher adsorption strength.

\subsection{Results and Discussion}

\subsubsection{Resin analysis}

The particle size, BET surface area, mean pore size and volume, water uptake and swelling are determined for all resins in order to relate the adsorption performances to the resin properties. The results are summarized in Table 3 . The BET surface area of M500 is not determined since this is a gel type resin.

Table 3: Resin properties.

\begin{tabular}{|c|c|c|c|c|c|c|}
\hline Resin & $\begin{array}{l}\text { Particle size } \\
\text { diameter } \\
(\mu \mathrm{m})\end{array}$ & $\begin{array}{c}\text { BET surface area } \\
\qquad\left(\mathrm{m}^{2} / \mathrm{g}_{\text {resin }}\right)\end{array}$ & $\begin{array}{c}\text { Mean } \\
\text { pore size } \\
(\mathrm{nm})\end{array}$ & $\begin{array}{c}\text { Pore } \\
\text { volume }(\mathrm{ml} / \\
\left.\mathrm{g}_{\text {resin }}\right) \text { at } \mathrm{p} / \mathrm{p}_{0} \\
=0.99\end{array}$ & $\begin{array}{l}\text { Wateruptake } \\
\left(\mathrm{ml}_{\mathrm{H} 2 \mathrm{O}} / \mathbf{g}_{\mathrm{dry} \text { resin }}\right)\end{array}$ & $\begin{array}{c}\text { Swelling } \\
\left(\mathrm{ml}_{\mathrm{H} 2 \mathrm{O}} / \mathrm{g}_{\mathrm{dry}}\right. \\
\text { resin })\end{array}$ \\
\hline XAD4 & 590 & $746.4 \pm 1.5$ & 6 & 1.11 & 2.4 & 0.1 \\
\hline Optipore & 557 & $1321.2 \pm 1.5$ & 56 & 0.85 & 2.8 & 1.1 \\
\hline MP62 & 625 & $39.3 \pm 0.2$ & 40 & 0.12 & 2.1 & 0.6 \\
\hline PVP & 366 & $53.7 \pm 0.1$ & 30 & 0.14 & 2.4 & 0.8 \\
\hline M500 $\left(\mathrm{OH}^{-}\right)$ & 660 & - & - & - & 2.6 & 2.3 \\
\hline M500 $\left(\mathrm{Cl}^{-}\right)$ & 585 & - & - & - & 0.9 & 1.3 \\
\hline
\end{tabular}


The particle size diameter determined is the diameter of the wet resins and is determined by the manufacturing method [30]. The particle size decreases in the following order: M500, MP62, XAD4, Optipore, PVP. The particle size is important for adsorption kinetics, but does not influence the adsorption capacity. It is therefore not further considered.

The BET surface area decreases in the following order: Optipore, XAD4, PVP and MP62. The BET surface area is high for the polymeric adsorbers Optipore and XAD4 and low for the ion exchange resins PVP and MP62. The surface areas of Optipore and XAD are comparable with the areas given by the manufacturers $\left(>1100 \mathrm{~m}^{2} / \mathrm{g}\right.$ and $750 \mathrm{~m}^{2} / \mathrm{g}$ respectively $\left.[22,23]\right)$. Surface areas of MP62 and PVP are not provided by the manufacturer. Because ion exchange resins are manufactured to contain many accessible ion exchange groups while the structure is $\mathrm{pH}$ resistant and capable to resist $\mathrm{pH}$ induced swelling and shrinkage, surface area is of minor importance compared to mechanical strength and ion exchange resins therefore have smaller surface areas. It is expected that when the chemical composition is similar, a larger surface area will lead to a higher adsorption capacity for the solute than when compared to a similar resin with a lower surface area. As such we expect Optipore to show a higher adsorption capacity for HMF than XAD4. The macroporous ion exchange resins MP62 and PVP show much lower BET surface areas and although they contain polar groups that could enhance HMF adsorption they are expected to show lower adsorption capacities.

The pore sizes of all resins are comparable except for XAD4 that shows a smaller pore size. The pore sizes are determined by the production method [30]. Pore sizes larger than $\sim 5 \mathrm{~nm}$ are suitable for the adsorption of small solutes because the solutes can migrate through the pores to occupy the entire surface area [31]. Since HMF is a small solute $(126.11 \mathrm{~g} / \mathrm{mol})$, all of these resins are suitable for HMF adsorption.

Pore volume from low to high is in the following order: MP62, PVP, Optipore and XAD4. M500 is a gel type resin and therefore contains no pores. Gel type resins possess higher loading capacities 
but have diffusion limitations in their adsorption kinetics. Macroporous resins show lower adsorption capacity but the adsorption sites are more readily available.

Water uptake of the resins shows, except for $\mathrm{M} 500\left(\mathrm{Cl}^{-}\right)$, only a little variation and is from low to high in the following order: MP62, PVP and XAD4 M500(OH-), Optipore. M500(Cl-) has a lower water uptake due to lower swelling of the matrix.

Swelling of the resins is from low to high in the following order: XAD4, MP62, PVP, Optipore, $\mathrm{M} 500\left(\mathrm{Cl}^{-}\right)$and $\mathrm{M} 500\left(\mathrm{OH}^{-}\right)$. Swelling of the resins is determined by the nature of the matrix, the degree of crosslinking, the macrostructure, the nature of the functional groups, the ion exchange capacity, and the nature of the counter ion [32]. Swelling of the resins can affect the adsorption capacity in different manners. A higher degree of swelling could influence the adsorption by making the adsorption sites better available [30]. Furthermore swelling increases the polymer volume thereby increasing the absorption area of the polymer matrix [30]. The water in the resins can be located in the pores, in the matrix of the resins or in a combination of both. In the first case no swelling is observed while in the second case swelling of the matrix is observed.

Although the water uptake of XAD4 is high, the swelling is very low. This indicates that the water present in the resin is mostly located in the pores of the resin. XAD4 consists of styrene-DVB and the apolar structure of the matrix explains the low swelling in water. It is generally accepted that water absorption in styrene-DVB polymers is due to the filling of the pores and not by swelling of the matrix [33, 34], although swelling in water has been observed when the polymerization occurred in a good solvent for the matrix [33].

The observed higher swelling of PVP and MP62 compared to XAD4 was expected since their matrix is less polar due to the introduction of the polar alkaline groups [32].

The relatively high swelling of Optipore is unexpected since, like XAD4, Optipore is specified as a apolar resin [23]. This is probably explained by the polymerization method. According to Xu et al. [30], Optipore is a hypercrosslinked polymer. These types of adsorbents are, after preparation in emulsion polymerization, further crosslinked with a crosslinker while swollen in a good solvent. The crosslinkers used for hypercrosslinking usually contain oxygen or chlorine containing reactive groups [30]. If the crosslinking reaction is incomplete these groups are still present in the polymer 
matrix thereby increasing the hydrophilicity of the polymer. Verne at al. [9] also mention that Optipore contains hydrophilic groups. Furthermore Yan et al. [33] observed that these hypercrosslinked resins, in contrast to the conventional macroporous resins, do swell in water at high degrees of crosslinking. This is caused by the inner strain caused by the high degree of crosslinking, which is released by swelling of the polymer. Although XAD4 and Optipore were chosen as resins with comparable chemical structure, these results show that this might not be the case. Not only the difference in surface area, but also the difference in degree of swelling, as well as the possibility of the presence of polar groups could affect the respective adsorption capacities.

M500 shows very high swelling due to the gel type structure (e.g. crosslinking) of the resin. The resin swells a lot more when $\mathrm{OH}^{-}$is chosen as counterion than when $\mathrm{Cl}^{-}$serves as counterion of the ammonium group, as the first counterion is known to cause the largest swelling of the resin [32]. Since $\mathrm{M} 500\left(\mathrm{OH}^{-}\right)$shows much more swelling than $\mathrm{M} 500\left(\mathrm{Cl}^{-}\right)$it is therefore expected that the HMF adsorption capacity of $\mathrm{M} 500\left(\mathrm{OH}^{-}\right)$is higher than that of $\mathrm{M} 500\left(\mathrm{Cl}^{-}\right)$.

We realize that the water mass balance does not add up. The water uptake $\left(\mathrm{ml} / \mathrm{g}_{\text {dry resin }}\right)$ is larger than the sum of the pore volume $\left(\mathrm{ml} / \mathrm{g}_{\text {dry resin }}\right)$ and the swelling $\left(\mathrm{ml} / \mathrm{g}_{\text {dry resin }}\right)$. This is likely due to the use of a combination of different techniques (e.g. nitrogen adsorption or water adsorption) and errors in the measurement. Nitrogen is a smaller molecule and can access smaller pores, at the same time swelling due to water affects pore volume and pore accessibility of some resins. Although the numbers do not fully match, the water uptake and swelling give a good idea of the mechanisms of water uptake (in the pore volume, due to swelling of the matrix or a combination of both).

\subsubsection{Adsorption isotherms}

HMF adsorption

HMF adsorption isotherms were determined to obtain information on the affinity and capacity of the different resins for HMF. The HMF adsorption isotherms are shown in Figure 1 for high HMF 
concentrations (1000-20,000 mg/l) and Figure 2 for low HMF concentrations (1-30 mg/l). The data are fitted with Langmuir (1000-20,000 mg/l) and Freundlich adsorption isotherms (1-30 mg/l). The fitted curves are shown in the figures as well and the adsorption isotherm parameters are given in Table 4.

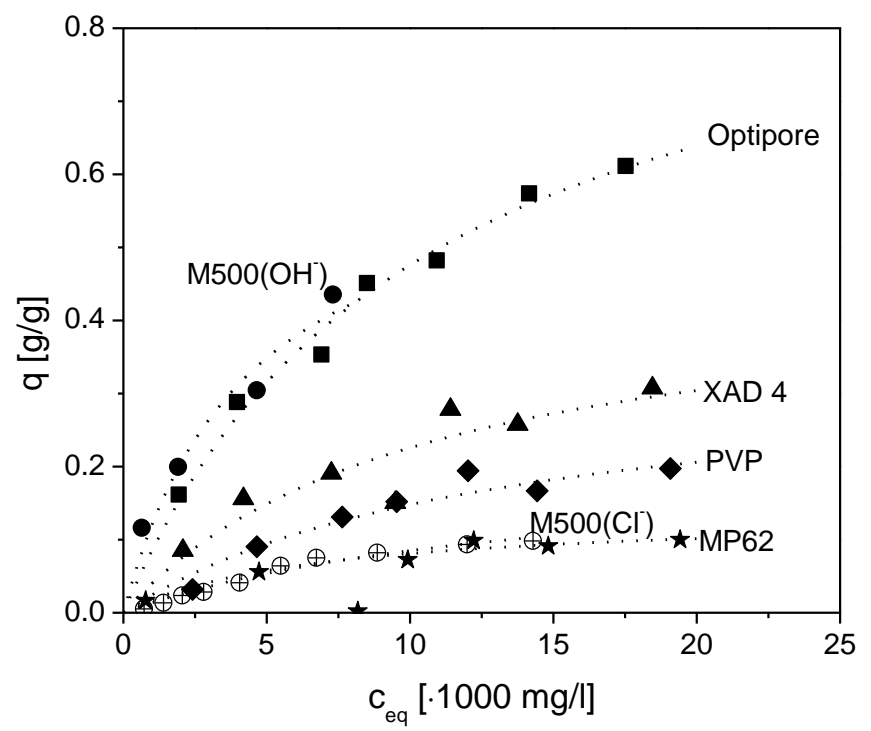

Figure 1: HMF adsorption isotherms $(0-20.000 \mathrm{mg} / \mathrm{l})$. The dotted lines show the fit according to the Langmuir isotherms, obtained Langmuir parameters are summarized in Table 4.

Figure 1 shows the adsorption isotherms for all resins of HMF at high concentrations. All resins show a type 1 Langmuir adsorption. Type 1 adsorption is limited to a single monolayer of adsorbate on the adsorbent surface characterized by saturation at high concentrations. Type 1 adsorption is often seen when the solvent has low affinity for the surface and does not compete for adsorption with the solute [35]. Type 1 Langmuir adsorption is also confirmed by the modeled data, since $\mathrm{R}^{2}$ is larger than 0.95 for all resins except for XAD4, which shows a lower $\mathrm{R}^{2}$ (see Table 6). Although XAD4 shows a lower $R^{2}$ due to more scatter in the data, the shape of the measured data still justifies the assumption of type 1 Langmuir adsorption. Langmuir type adsorption of furans on styrene-DVB resins with and without anion exchange groups was also observed by several others $[6,16,36]$. 
The adsorption capacity of the resins increases in the following order: MP62, M500( $\left.\mathrm{Cl}^{-}\right)$, PVP, XAD4, M500(OH') and Optipore. The adsorption isotherms of MP62 and M500(Cl-) show the lowest adsorption capacity and are saturated at the measured concentrations. MP62 has a low surface area and therefore less available adsorption sites. $\mathrm{M} 500\left(\mathrm{Cl}^{-}\right)$swells only little and adsorption sites cannot be reached by diffusion through the gel. Since no other groups than aromatic rings are present in the matrices of XAD4 and Optipore, adsorption of HMF on Optipore and XAD is established by $\pi-\pi$ interactions between the styrene matrix and the ring of HMF. Adsorption of the polar groups of HMF on the apolar matrix of XAD or Optipore of HMF is unlikely. XAD4 shows a lower adsorption capacity than Optipore. This is related to the surface area of the resins. The surface area of Optipore is $1321.2 \mathrm{~m}^{2} / \mathrm{g}$ while the surface area of XAD4 is $746.4 \mathrm{~m}^{2} / \mathrm{g}$ only. The ratio of maximum adsorption capacity to BET surface area is $0.63 \mathrm{mg} \mathrm{HMF}_{\mathrm{HF}} / \mathrm{m}^{2}$ and $0.73 \mathrm{mg} \mathrm{HMF}_{\mathrm{HF}} / \mathrm{m}^{2}$ for XAD4 and Optipore respectively. Since the swelling experiments showed that Optipore swells while XAD4 does not, we believe that this causes the relatively higher HMF adsorption since swelling increases the adsorption the adsorption sites become better accessible [30].

The adsorption capacities of the macroporous anion exchange resins MP62 and PVP is much lower than that of Optipore and XAD4. This is explained by the small surface area of these resins.

$\mathrm{M} 500\left(\mathrm{OH}^{-}\right)$shows a very high adsorption capacity while $\mathrm{M} 500\left(\mathrm{Cl}^{-}\right)$shows a very low adsorption capacity. This is caused by the swelling of the resin, which depends on the counter ion. $\mathrm{M} 500(\mathrm{Cl}-)$ swells only little while $\mathrm{M} 500\left(\mathrm{OH}^{-}\right)$has a huge degree of swelling. This is directly reflected in a lower adsorption capacity.

The affinity of the resins could provide more insight in the HMF adsorption mechanism of the resins. It is clear that HMF adsorbs on XAD4 and Optipore by $\pi-\pi$ bonds since no other interactions are available. For the other resins an increased or reduced affinity compared to XAD4 and Optipore is observed, depending on the affinity of the resin for HMF. The affinity of the resins is indicated by the affinity parameter $b$ of the Langmuir isotherm and is determined by the slope of the curve at lower equilibrium concentration ranges when the curve is still linear. Values of $b$ were obtained by fitting the data of Figure 1 with Langmuir (see Table 4). The obtained values for $b$ are comparable for all resins. Since there are not so many data points in the linear part of the graph 
that determine $b$, the value of $b$ is further investigated by analyzing the adsorption isotherms at low concentrations (see Figure 2).

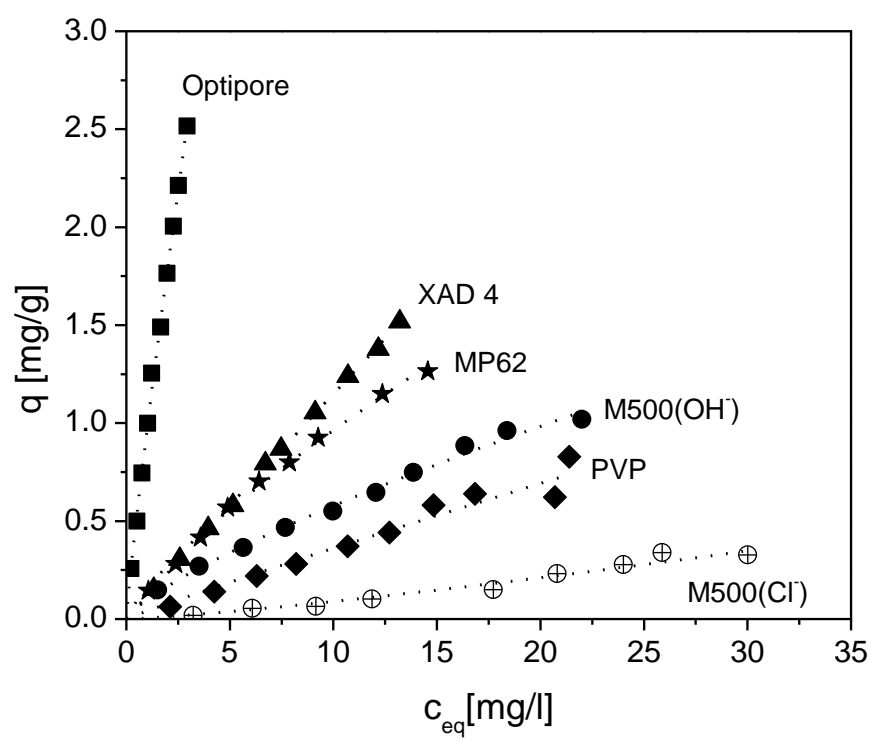

Figure 2: HMF adsorption isotherms [0-30 mg/l]. The dotted lines show the fit according to Freundlich (The obtained Freundlich parameters are summarized in Table 4).

Figure 2 shows the adsorption isotherms of the resin at low concentrations. The adsorption capacity of the resins for HMF increases in the following order: M500(Cl'), PVP, M500(OH'), MP62, XAD4 and Optipore. 1/n and $\mathrm{K}$ are obtained by modeling of the data with Freundlich (Equation 4 and Table 4). Although the data fitted with Freundlich describe the adsorption isotherms well, $1 / n$ and $\mathrm{K}$ have no physical meaning in this case, as the isotherms are mostly linear and $1 / \mathrm{n}$ and $\mathrm{K}$ are correlated.

However, when the maximum capacity $\left(q_{\max }\right)$ obtained from the adsorption isotherms at high concentrations (Figure 1) is used, more information on the affinity at low concentrations can be obtained. For low concentrations $\left(\mathrm{b}^{\circ} \mathrm{C}_{\mathrm{eq}}<<1\right)$ Equation 3 can be simplified to the linear correlation (Equation 5):

$\frac{q}{q_{\max }}=b \cdot c_{e q}$ 
The adsorption capacity (q) measured at low concentrations (Figure 2) is divided by the maximum adsorption capacity $\left(\mathrm{q}_{\max }\right)$ obtained at high concentrations (Table 4). Figure 2 was recalculated using the maximum adsorption capacity and the following figure was obtained (Figure 3). Equation 5 shows that the slope of the adsorption isotherms is equal to the affinity parameter (b).

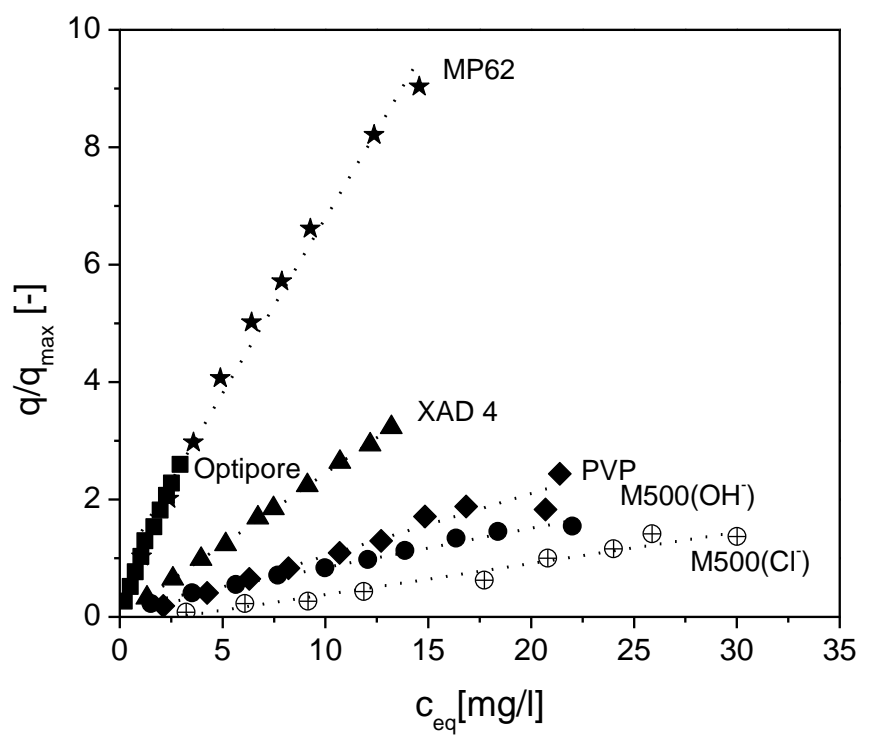

Figure 3: HMF adsorption isotherms (0-30 mg/l). The dotted lines show the fit based on the linear correlation (the obtained parameters are summarized in Table 4).

The data fit the linear correlation well since $R^{2}>0.95$ (Table 6). The slope and therefore the affinity is highest for Optipore immediately followed by MP62 and subsequently XAD4, PVP, M500(OH') and $\mathrm{M} 500(\mathrm{Cl})$. Since the adsorption isotherm is corrected for the maximum capacity, the resin swelling and surface area have no effect on the slope of the graph. Optipore mainly consists of a styrene-DVB matrix and since the affinity of HMF is largest for this resin, it shows that $\pi-\pi$ stacking is the main contributor to HMF adsorption. XAD4 contains the same chemical composition as Optipore but shows a lower affinity for HMF. This is caused by the altered morphology of the hypercrosslinked Optipore compared to that of XAD4 as was shown before by Jerabek et al. for comparable resins [36], who showed that the micropores formed by hypercrosslinking enhance the adsorbence at low concentration similar to adsorption in micropores in gas-solid adsorption. 
It appears that the addition of a tertiary amine enhances the affinity of HMF for the resin since the affinity of MP62 is higher than that of XAD4 and almost comparable to Optipore. It is possible that the polar groups of HMF form hydrogen bonds with the tertiary amine of MP62, as was suggested by Fargues et al. [16] as a mechanism for furfural adsorption on tertiary amines. The adsorption mechanism of HMF on MP62 is further discussed when glucose adsorption and competitive adsorption is evaluated.

Addition of a pyridine to the matrix reduces the affinity of the resins for HMF, as the affinity of PVP is lower than that of XAD4 and Optipore. We expected the bulky aromatic group to show $\pi-\pi$ stacking with HMF and hydrogen bond formation between the polar groups of HMF with the amine of the pyridine, thereby enhancing the affinity. However the reduced affinity compared to XAD4 and Optipore shows a reduction upon addition of a pyridine group. We hypothesize that since the pyridine group does not seem to contribute to HMF adsorption, it blocks the access to the styrene matrix thereby reducing HMF adsorption, as was also considered for other ion exchange resins by Fargues et al. [16].

Addition of strong ion exchange groups such as in $\mathrm{M} 500\left(\mathrm{OH}^{-}\right.$and $\left.\mathrm{Cl}^{-}\right)$also does not enhance the affinity of the materials for HMF as these resins show very low affinity constants. The slope of $\mathrm{M} 500\left(\mathrm{OH}^{-}\right)$is slightly higher than that of $\mathrm{M} 500\left(\mathrm{Cl}^{-}\right)$but we believe that is caused by the value of $q_{\max }$ that is very different for both resins and susceptible to a certain error. The affinities of the resins are therefore comparable and differences in adsorption capacity are likely caused by different swelling due to the different counter ions. The reduced affinity of M500 compared to the other styrene-DVB resins might also be caused by steric hindrance of the styrene matrix by the quaternary amine.

The results on the HMF adsorption capacity (low and high concentration) show that the main factor influencing HMF adsorption capacity of the styrene-DVB resins is the surface area. The main mechanism for HMF adsorption for all resins is $\pi-\pi$ stacking with the styrene-DVB matrix. It appears that the addition of polar anion exchange groups reduce the affinity, probably caused by steric hindrance. In the case of the tertiary amine, the adsorption mechanism is less obvious. It is possible that HMF adsorption is caused by $\pi-\pi$ stacking or hydrogen bonding or a combination of both. 
Table 4: Adsorption isotherm parameters obtained from the data in Figure 1, 2 and 3.

\begin{tabular}{|c|c|c|c|c|c|c|c|c|}
\hline & Langmuir & & & Freundlich & & & $\begin{array}{l}\text { Linear } \\
\text { correlation }\end{array}$ & \\
\hline & $\begin{array}{l}\text { b } \\
\left(\mathrm{l} / \mathrm{g}_{\text {HMF }}\right)\end{array}$ & $\begin{array}{l}\mathbf{q}_{\max } \\
\left(\mathbf{g}_{\text {нMF }} / \mathbf{g}_{\text {resin }}\right)\end{array}$ & $\mathbf{R}^{2}$ & $\begin{array}{l}\mathrm{K} \\
\left(\mathrm{l} / \mathrm{g}_{\mathrm{resin}}\right)\end{array}$ & $1 / n$ & $\mathbf{R}^{2}$ & b (I/mg & $\mathbf{R}^{2}$ \\
\hline XAD 4 & 0.09 & 0.47 & 0.82 & 0.12 & 0.98 & 1.00 & 0.24 & 0.99 \\
\hline Optipore & 0.10 & 0.97 & 0.98 & 0.97 & 0.89 & 1.00 & 0.86 & 0.99 \\
\hline MP 62 & 0.15 & 0.14 & 0.97 & 0.16 & 0.78 & 1.00 & 0.60 & 0.98 \\
\hline PVP & 0.08 & 0.34 & 0.92 & 0.04 & 0.94 & 0.95 & 0.11 & 0.96 \\
\hline $\mathrm{M} 500\left(\mathrm{OH}^{-}\right)$ & 0.22 & 0.66 & 0.95 & 0.10 & 0.77 & 0.99 & 0.07 & 0.99 \\
\hline $\mathrm{M} 500\left(\mathrm{Cl}^{-}\right)$ & 0.07 & 0.20 & 0.98 & 0.01 & 1.23 & 0.95 & 0.05 & 0.95 \\
\hline
\end{tabular}




\section{Glucose adsorption}

In the targeted industrial application glucose is abundantly present while HMF is only present in trace amounts. As such, undesired glucose adsorption may affect HMF adsorption by competitive adsorption if both components exhibit similar adsorption mechanisms. Additionally sugar loss due to adsorption of sugars (e.g. glucose) reduces the final fermentation yield. Glucose adsorption for all resins was therefore studied as well. The glucose adsorption isotherms of $\mathrm{M} 500\left(\mathrm{OH}^{-}\right)$and MP62 are given in Figure 4. Glucose adsorption was not observed for XAD4, PVP, M500(Cl) and Optipore.

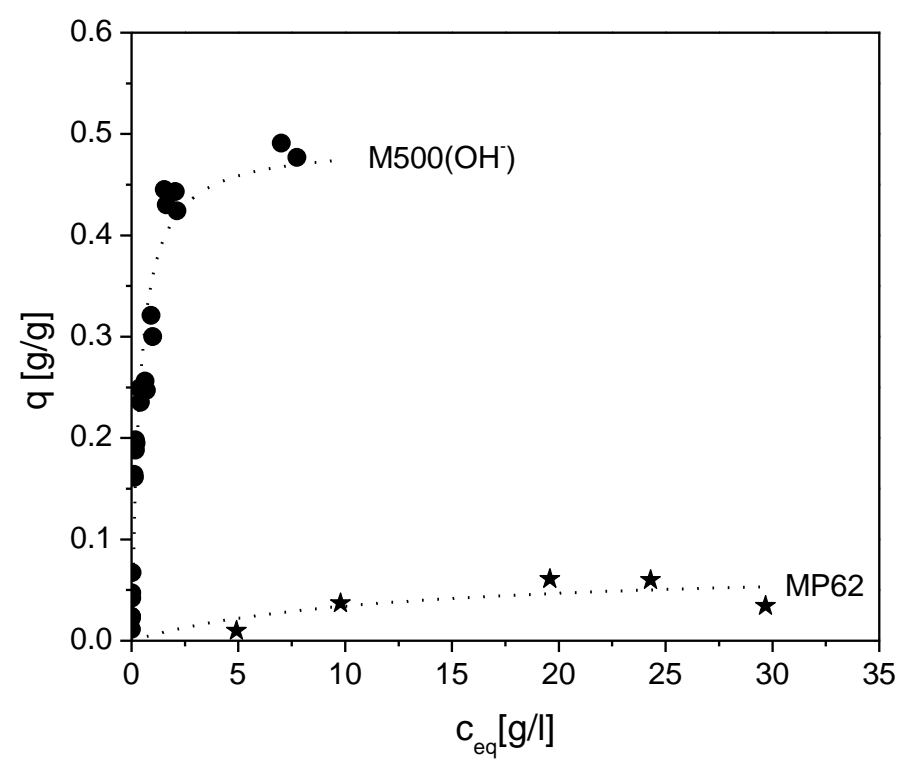

Figure 4: Glucose adsorption isotherms of $\mathrm{M} 500\left(\mathrm{OH}^{-}\right)$and MP62 ([c $\left.\left.\mathrm{c}_{\text {eq glucose }}\right]=0-30 \mathrm{~g} / \mathrm{l}\right)$.

Figure 4 shows that the weak and strong ion exchange resins MP62 and M500(OH $)$ show glucose adsorption. Since glucose adsorption was not observed for XAD4 and Optipore, adsorption does not occur at the styrene-DVB matrix of the resins. This is in agreement with Weil et al. [6]. The absence of glucose adsorption on PVP is in line with the work of Xie et al [11]. However these studies were both performed with column experiments. The observed glucose adsorption on MP62 is most probably due to hydrogen bonding of the alcohol group of glucose to the tertiary amine. Saari et al. [37] also observed glucose adsorption on a weak anion exchanger. The amount of glucose adsorption at these concentrations was comparable but the curve did not reach maximum adsorption and was linear instead of following Langmuir. This could be related to differences in 
physical properties (e.g. pore size, surface area) of the resin between this work and the work of Saari et al., which are not known. Although we believe that glucose binds to MP62 through hydrogen bonding we do not observe glucose adsorption on PVP.

Although glucose is known not to adsorb on strong ion exchange resins [38], examples of glucose adsorption on strong ion exchange resin in their $\mathrm{OH}^{-}$form were found $[17,39]$. The mechanism of this glucose adsorption on a quaternary ammonium countered with $\mathrm{OH}^{-}\left(\mathrm{M} 500\left(\mathrm{OH}^{-}\right)\right)$is however unclear since hydrogen bonding with a quaternary ammonium is not possible [40]. According to Nilvebrant et al. [17] glucose adsorption is caused by the tendency of glucose to ionize and form ionic bonds with quaternary ammonium. We believe that since glucose is a weak acid (pKa >14 [21]) this mechanism might be an explanation for glucose adsorption at high $\mathrm{pH}$, but it does not explain the adsorption of glucose at neutral $\mathrm{pH}$, as was measured in this research and at $\mathrm{pH} 5.5$ by Nilvebrant et al. [17]. Since glucose adsorption is not observed for the $\mathrm{Cl}^{-}$form (this research) or the $\mathrm{SO}_{4}{ }^{2-}$ form (Nilvebrandt et al. [17]) of the ammonium salt, hydrogen bonding with the counter ion $\mathrm{OH}^{-}$can be an explanation for glucose adsorption on $\mathrm{M} 500\left(\mathrm{OH}^{-}\right)$.

The very high glucose adsorption on $\mathrm{M} 500\left(\mathrm{OH}^{-}\right)$compared to MP62 is due to the different matrix structure (e.g. gel type vs macroporous). A higher degree of crosslinking tightens structure of the matrix, making this less accessible for the large hydrate glucose molecule as was also shown for cation exchange resins [41-44].

\section{Competitive adsorption}

The effect of the presence of glucose on HMF adsorption was studied as well to examine if glucose and HMF compete for adsorption sites. As described, the main mechanism for HMF adsorption of all resins is $\pi-\pi$ stacking, however hydrogen bonding may play a role for the resin MP62. To verify the importance of competitive adsorption of HMF and glucose Figure 5 shows the HMF adsorption isotherms in the presence of glucose determined for XAD4 and Optipore. It was not possible to determine the HMF adsorption on MP62, PVP and M500 at low HMF concentrations as contamination of the resins related to the production of the resins hindered HMF analysis for the ion exchange resins at low concentration. Although the anion exchange resins were very 
intensively rinsed with water before the adsorption experiments until contaminants were no longer visible with UV-VIS, when the resins were exposed to the glucose solution in the adsorption experiment they released additional contaminants. This combined with the presence of glucose hindered accurate HMF analysis at this concentration range. However in case competitive adsorption occurs, this would also be visible at high concentrations. Therefore HMF adsorption for M500( $\left(\mathrm{OH}^{-}\right)$, PVP and MP62 in the presence of glucose was determined at higher HMF concentrations $(0-20,000 \mathrm{mg} / \mathrm{l})$ in order to be still able to evaluate the contribution of competitive adsorption between HMF and glucose. These data are represented in Figure 6.

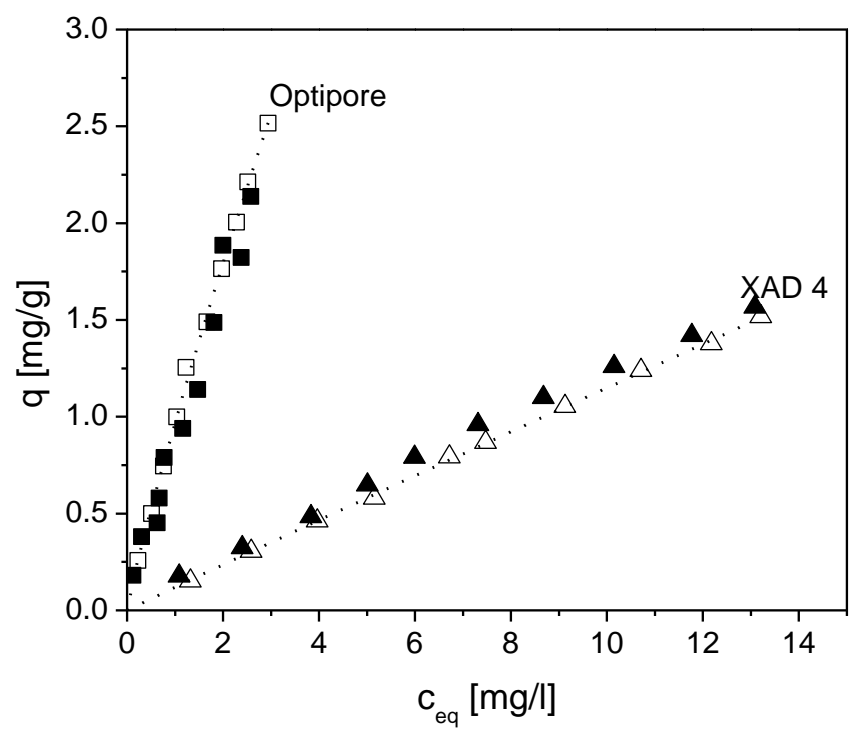

Figure 5: HMF adsorption in water (open symbols) and the presence of glucose ( $\left.\mathrm{c}_{\text {glucose }}=100 \mathrm{~g} / \mathrm{l}\right)$ (closed symbols) for Optipore and XAD $4\left(\left[\mathrm{C}_{\mathrm{eq} \mathrm{HMF}}\right]=0-14 \mathrm{mg} / \mathrm{l}\right)$. 


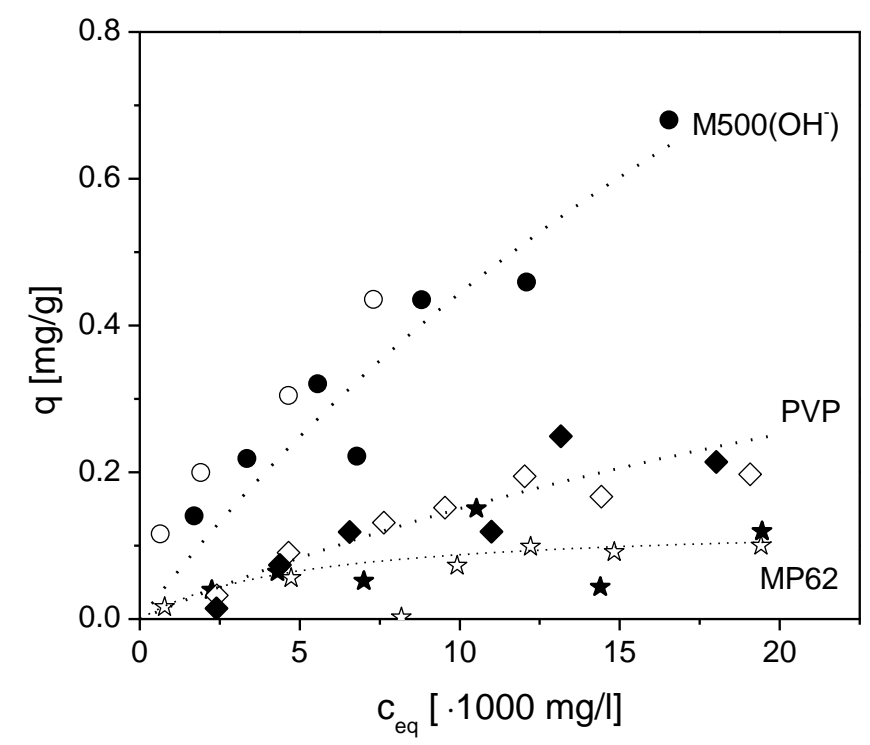

Figure 6: HMF adsorption in water (open symbols) and the presence of glucose ( c glucose $_{1}=100 \mathrm{~g} / \mathrm{l}$ ) (closed symbols)for M500, PVP, MP62 ([C $\left.\left.\mathrm{C}_{\text {eq HMF }}\right]=0-20.000 \mathrm{mg} / \mathrm{l}\right)$.

Figure 5 clearly shows that for the two resins without specific functional groups, competitive adsorption is essentially not observed. Independent on the presence of glucose or not, the same amount of HMF is adsorbed over the full HMF concentration range investigated. The resins $\mathrm{M} 500\left(\mathrm{OH}^{-}\right), \mathrm{PVP}$ and MP62 show similar behavior (Figure 6), however the adsorption isotherms show more scatter. As discussed earlier this is caused by contaminants, related to the production of the resins and released from the anion exchange resins.

The absence of competitive adsorption on M500 and MP62 shows that the adsorption mechanism for HMF differs from that of glucose, which confirms that HMF adsorption is established through $\pi-\pi$ interactions. We believe that glucose adsorption on MP62 is established through hydrogen bonding with the tertiary amine. Although HMF has an alcohol and a keton group that could bind through hydrogen bonds to MP62 as well, the absence of competitive adsorption shows that this mechanism does not play a role in HMF adsorption on MP62. This result also confirms that HMF adsorption for all resins is predominantly through $\pi-\pi$ bonding with the styrene matrix.

Fargues et al. [16] opt that furfural adsorption on a strong anion exchange resin with a styrene matrix is due to hydrogen bonding of the polar keton group of furfural to the quaternary amine. We believe however that the only possibility for hydrogen bonding is the $\mathrm{OH}^{-}$counter ion of the 
quaternary ammonium. We believe that glucose binds to $\mathrm{M} 500\left(\mathrm{OH}^{-}\right)$through hydrogen bonding and since competitive adsorption between glucose and HMF is not observed, hydrogen bonding does not play a role in HMF adsorption.

All competitive adsorption isotherm graphs show that the presence of glucose does not affect the HMF adsorption to the resins. Additionally, this also shows that the presence of glucose does not alter the solubility of HMF, since the adsorption to the resins is not affected by the presence of glucose.

\subsubsection{Temperature effect}

The HMF adsorption isotherms show that styrene-DVB based resins are good adsorbers for HMF. The affinity of these adsorbers for HMF is high, however this could make desorption difficult. Regeneration with steam is a common method to desorb solutes from a column [45]. Furthermore the pretreatment methods to extract sugars from lignocellulosic mass often include a temperature increase (e.g. liquid hot water extraction, steam explosion) that affects the adsorption thermodynamics. So additionally we investigated the effect of temperature on HMF adsorption for Optipore at $20^{\circ} \mathrm{C}$ and at $60^{\circ} \mathrm{C}$ (Figure 7).

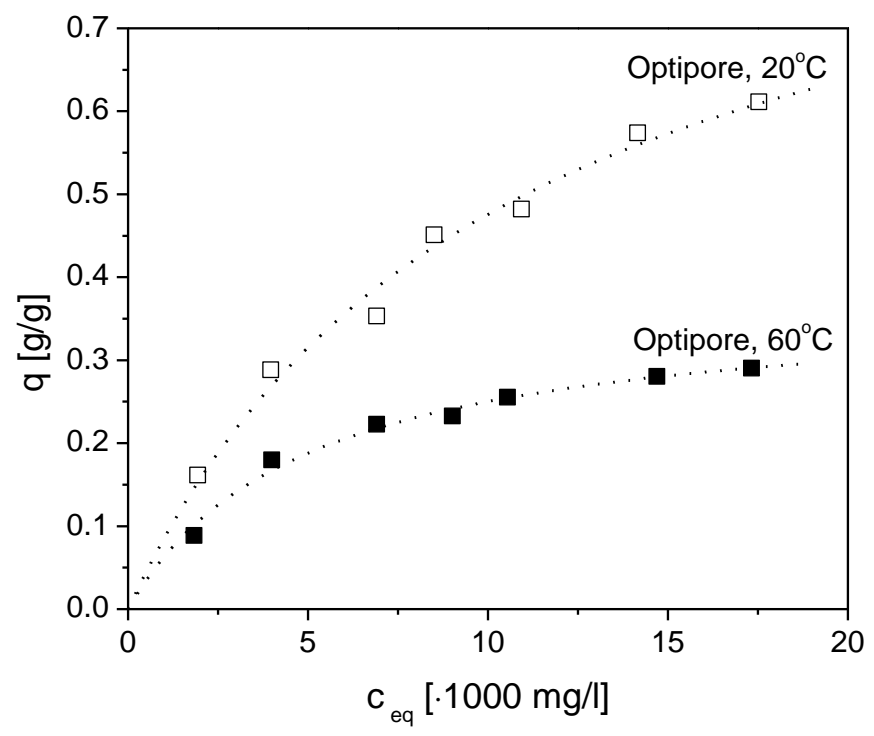

Figure 7: HMF adsorption on Optipore at $20^{\circ} \mathrm{C}$ and $60^{\circ} \mathrm{C}\left(\left[\mathrm{C}_{\mathrm{eq} \mathrm{HMF}}\right]=0-20.000 \mathrm{mg} / \mathrm{l}\right)$. 
Figure 7 clearly shows that the adsorption capacity of Optipore for HMF is higher at lower temperatures. This confirms that adsorption is established by physical adsorption. This effect was also observed for XAD4 by Weil et al. [6]. The efficiency of the adsorbers at higher temperatures is reduced, but this reduced affinity can also be used to regenerate the column after saturation by increasing the temperature. This is especially effective at higher HMF concentrations.

\title{
3.5 Conclusions
}

Furan derivatives can be removed from water and sugar solutions with styrene based (ion exchange) polymeric resins. For efficient removal, a high surface area of the resin is a key property. Although introduction of polar groups in the form of tertiary amines appears to increase the affinity of the resin material for HMF, these groups also introduce affinity for glucose resulting in loss of fermentable sugar. However the presence of glucose did not affect the HMF adsorption capacity and solubility and competition between HMF and glucose for adsorption sites was not observed in the ion exchange resins. HMF adsorption is likely established by $\pi-\pi$ interactions between the styrene matrix and the ring of the furan, while glucose adsorption is predominantly by hydrogen interactions with the polar groups of the ion exchange groups. Optipore shows a high specificity for HMF adsorption while it does not exhibit specificity for glucose. This makes it an excellent adsorber for HMF removal from hydrolysate for the fermentation of glucose.

\subsection{Abbreviations and symbols}

\author{
Abbreviations
}

DLS Dynamic light scattering

DVB Divinylbenzene

HMF 5-Hydoxymethylfurfural

$\mathrm{H}_{2} \mathrm{SO}_{4} \quad$ Sulfuric acid

MP62 Lewatit MP 62

M500(Cl- Lewatit M500 with counterion $\mathrm{Cl}^{-}$ 


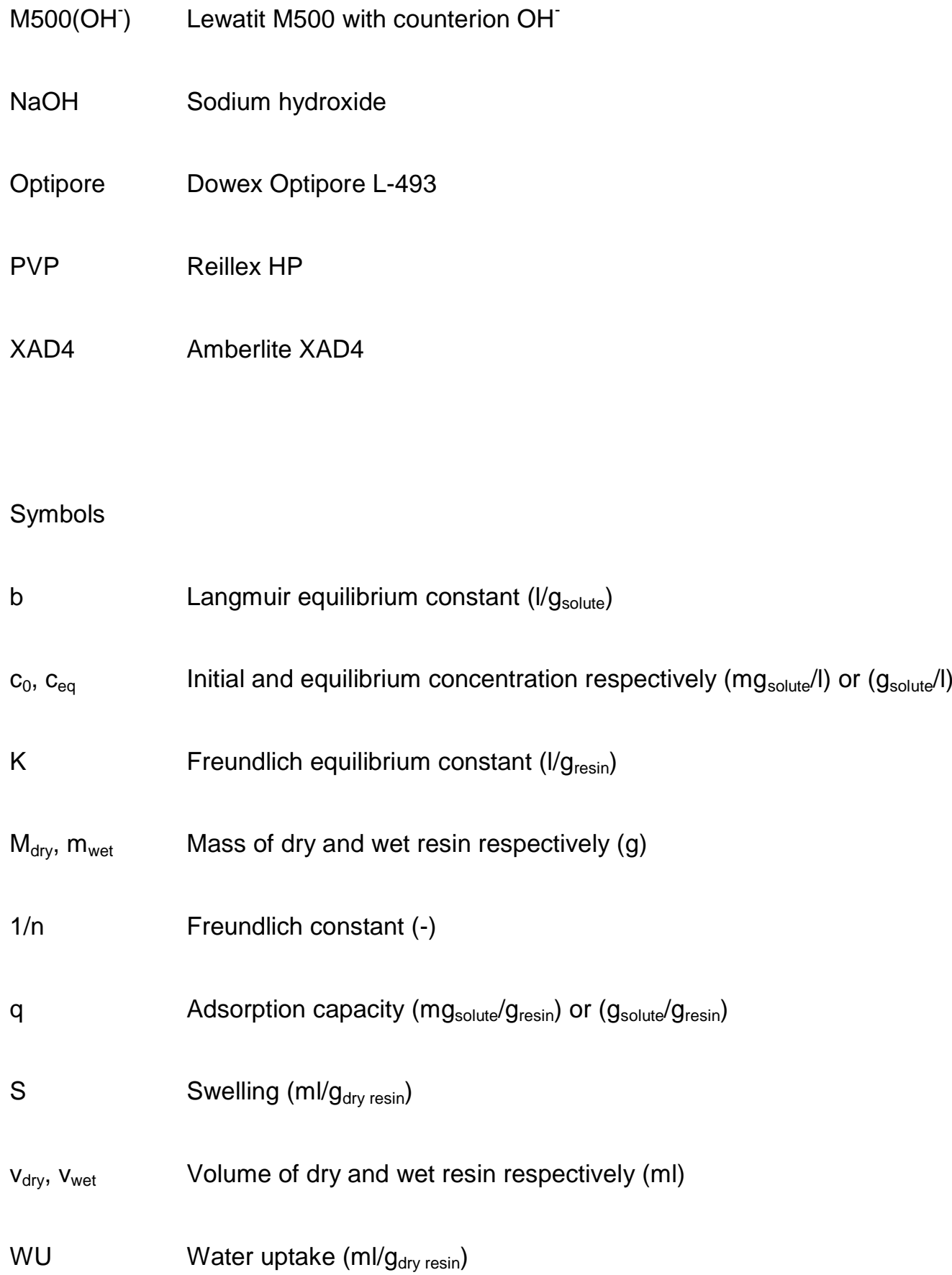

\subsection{Acknowledgment}

This work was carried out within project SC-00-04 of the Institute for Sustainable Process Technology (ISPT), The Netherlands. 


\subsection{References}

1. Biorefenery. Available from: $h$ ttp://www.biorefinery. $n l$. [cited 2011 july 8].

2. Ranjan, R., et al., Adsorption of fermentation inhibitors from lignocellulosic biomass hydrolyzates for improved ethanol yield and value-added product recovery. Microporous and Mesoporous Materials, 2009. 122(1-3): p. 143-148.

3. Mancilha, I.M.d. and M.N. Karim, Evaluation of Ion Exchange Resins for Removal of Inhibitory Compounds from Corn Stover Hydrolyzate for Xylitol Fermentation. Biotechnol. Prog., 2003. 19: p. 1837-1841.

4. Mills, T.Y., N.R. Sandoval, and R.T. Gill, Cellulosic hydrolysate toxicity and tolerance mechanisms in Escherichia coli. Biotechnology for Biofuels, 2009. 2.

5. Huang, H.J., et al., A review of separation technologies in current and future biorefineries. Separation and Purification Technology, 2008. 62(1): p. 1-21.

6. Weil, J.R., et al., Removal of fermentation inhibitors formed during pretreatment of biomass by polymeric adsorbents. Industrial \& Engineering Chemistry Research, 2002. 41(24): p. 6132-6138.

7. Nanoti, A., et al., Process studies for development of adsorption technology for furfural recovery from waste water. Environmental Progress, 2008. 27(3): p. 308-312.

8. Gupta, P., et al., The removal of furfural from water by adsorption with polymeric resins. Separation Science and Technology, 2001. 36(13): p. 2835-2844.

9. Vern, C., et al., The beet sugar factory of the future. International Sugar Journal, 1995. 97(1159): p. 310-312, 314, 316.

10. de Carvalho, W., et al., Detoxification of sugarcane bagasse hemicellulosic hydrolysate with ion-exchange resins for xylitol production by calcium alginate-entrapped cells. Journal of Chemical Technology \& Biotechnology, 2004. 79(8): p. 863-868.

11. Xie, Y., et al., Comparison of two adsorbents for sugar recovery from biomass hydrolyzate. Industrial \& Engineering Chemistry Research, 2005. 44(17): p. 6816-6823.

12. Maciel de Mancilha, I. and M.N. Karim, Evaluation of ion exchange resins for removal of inhibitory compounds from corn stover hydrolyzate for xylitol fermentation. Biotechnology progress, 2003. 19(6): p. 1837-1841.

13. Canilha, L., J.o.B. de Almeida e Silva, and A.I.N.p. Solenzal, Eucalyptus hydrolysate detoxification with activated charcoal adsorption or ion-exchange resins for xylitol production. Process Biochemistry, 2004. 39(12): p. 1909-1912.

14. Singh, S., V.C. Srivastava, and I.D. Mall, Fixed-bed study for adsorptive removal of furfural by activated carbon. Colloids and Surfaces A: Physicochemical and Engineering Aspects, 2009. 332(1): p. 50-56.

15. Alves, L.A., et al., Pretreatment of sugarcane bagasse hemicellulose hydrolysate for $x y l i t o l$ production by Candida guilliermondii. Applied Biochemistry and Biotechnology, 1998. 70-2: p. 89-98.

16. Fargues, C., R. Lewandowski, and M.-L. Lameloise, Evaluation of ion-exchange and adsorbent resins for the detoxification of beet distillery effluents. Industrial \& Engineering Chemistry Research, 2010. 49(19): p. 9248-9257.

17. Nilvebrant, N.-O., et al., Detoxification of lignocellulose hydrolysates with ion-exchange resins. Applied Biochemistry and Biotechnology, 2001. 91-93(1-9): p. 35-49.

18. Dow, Dowex ion exchange resins, Dowex optipore SD-2 adsorbent as an alternative to activated carbon in the processing of corn syrups and high fructose corn syrups, 1998.

19. Schubert, J., et al., Waterpipe smoke: A considerable source of human exposure against furanic compounds. Analytica Chimica Acta, 2012. 709(0): p. 105-112.

20. Mazzobre, M.F., et al., Octanol-water partition coefficient of glucose, sucrose, and trehalose. Carbohydrate Research, 2005. 340(6): p. 1207-1211.

21. Feng, S.T., C. Bagia, and G. Mpourmpakis, Determination of Proton Affinities and Acidity Constants of Sugars. Journal of Physical Chemistry A, 2013. 117(24): p. 5211-5219.

22. RohmHaas, Amberlite XAD4, industrial grade polymeric adsorber, product data sheet, 2003.

23. Dow, Product information Dowex Optipore L493 and V493. Product information Dowex Optipore L493 and V493.

24. Lanxess, Product information Lewatit MP 62. Product information Lewatit MP 62, 2010.

25. Vertellus, Specifications Reillex HP Polymer. 
26. Lanxess, Product information Lewatit Monoplus M 500. Product information Lewatit Monoplus M 500, 2010.

27. Ramaswamy, S., H.-J. Huang, and B. Ramarao, in Separation and Purification Technologies in Biorefineries. 2013, John Wiley \& Sons, Ltd. p. 1-36.

28. Sing, K., The use of nitrogen adsorption for the characterisation of porous materials. Colloids and Surfaces A: Physicochemical and Engineering Aspects, 2001. 187-188(0): p. 3-9.

29. Thomas, W.J.C.B.D., Adsorption technology and design. 1998, Oxford; Boston: Butterworth-Heinemann.

30. Xu, Z., Q. Zhang, and H.H.P. Fang, Applications of Porous Resin Sorbents in Industrial Wastewater Treatment and Resource Recovery. Critical Reviews in Environmental Science and Technology, 2003. 33(4): p. 363-389.

31. Aldrich, S., Amberlite XAD polymeric resins, product information. 1998.

32. Zagorodni, A.A. Ion exchange materials : properties and applications. 2007.

33. Yan, J., X. Wang, and J. Chen, Swelling of porous styrene-divinylbenzene copolymers in water. Journal of Applied Polymer Science, 2000. 75(4): p. 536-544.

34. Millar, J.R., et al., 33. Solvent-modified polymer networks. Part I. The preparation and characterisation of expanded-network and macroporous styrene-divinylbenzene copolymers and their sulphonates. Journal of the Chemical Society (Resumed), 1963. 0(0): p. 218-225.

35. Giles, C.H., A.P. D'Silva, and I.A. Easton, A general treatment and classification of the solute adsorption isotherm part. II. Experimental interpretation. Journal of Colloid and Interface Science, 1974. 47(3): p. 766-778.

36. Jerabek, K., L. Hankova, and Z. Prokop, Post-crosslinked polymer adsorbents and their properties for separation of furfural from aqueous solutions. Reactive Polymers, 1994. 23(2-3): p. 107-112.

37. Saari, P., H. Heikkila, and M. Hurme, Adsorption equilibria of arabinose, fructose, galactose, glucose, mannose, rhamnose, sucrose, and xylose on ion-exchange resins. Journal of Chemical and Engineering Data, 2010. 55(9): p. 3462-3467.

38. Khym, J.X. and L.P. Zill, The separation of sugars by ion exchange. Journal of the American Chemical Society, 1952. 74(8): p. 2090-2094.

39. Saska, M. and M. Diack, Separation of inositols from sugars and sugar alcohols, 1996, Board of supervisors of Louisiana state university and agricultural and mechanical college.

40. Spath, A. and B. Konig, Molecular recognition of organic ammonium ions in solution using synthetic receptors. Beilstein Journal of Organic Chemistry, 2010. 6: p. 32.

41. Caruel, $\mathrm{H}$., et al., Chromatographic separation of polyols by ligand exchange: Effects of the ion-exchange resin cross-linking and size. Journal of Chromatography A, 1992. 594(1-2): p. $125-130$.

42. Vente, J.A., et al., Comparison of sorption isotherms of mono- and disaccharides relevant to oligosaccharide separations for $\mathrm{Na}, \mathrm{K}$, and $\mathrm{Ca}$ loaded cation exchange resins. Chemical Engineering Communications, 2005. 192(1): p. 23-33.

43. Lei, H., et al., Adsorption behavior of glucose, xylose, and arabinose on five different cation exchange resins. Journal of Chemical \& Engineering Data, 2009. 55(2): p. 735-738.

44. Vankova, K., M. Gramblika, and M. Polakovic, Single-component and binary adsorption equilibria of fructooligosaccharides, glucose, fructose, and sucrose on a Ca-form cation exchanger. Journal of Chemical \& Engineering Data, 2009. 55(1): p. 405-410.

45. RohmHaas, Amberlite XAD polymeric adsorbers, 2000. 


\section{Adsorption kinetics of Dowex $^{\mathrm{TM}}$ Optipore $^{\mathrm{TM}}$ L493 for the removal of the furan 5-hydroxymethylfurfural from}

\section{sugar}

Journal of Chemical Technology \& Biotechnology, 2015

\subsection{Abstract}

Background: Recently much research focused on the production and refinery of biobased fuels. The production of biofuels derived from lignocellulosic biomass is recognized as a promising route to produce biobased fuels responsibly. Often, product streams (e.g. glucose) still contain small amounts of undesired components (e.g.furans such as HMF). This study focusses on the removal of furans produced during the fermentation. In earlier work, styrene based resins have been identified as promising materials for this separation. In this work we study the kinetic properties of the most promising resin: Dowex ${ }^{\mathrm{TM}}$ Optipore ${ }^{\mathrm{TM}} \mathrm{L} 493$.

Results: The diffusion coefficient of $5 \mathrm{mg} / \mathrm{HMF}$ was $\sim 810^{-12} \mathrm{~m}^{2} / \mathrm{s}$ in water and $3.010^{-12} \mathrm{~m}^{2} / \mathrm{s}$ in a glucose solution. The reduced diffusion coefficient in the particle when glucose is present is caused by the higher viscosity of the glucose solution and it indicates that diffusion is controlled by surface and pore diffusion. The breakthrough curves of HMF on Optipore showed that the column is very efficient at the conditions of interest.

Conclusion: This study shows that Optipore is a much more efficient resin for HMF removal than currently used resins. Its fast kinetics and capacity make it possible to efficiently remove HMF from glucose solutions.

Key words: HMF, glucose, breakthrough, zero length column, biorefinery, Optipore L493. 


\subsection{Introduction}

Recently much research focused on the production and refinery of biobased fuels. The production of biofuels derived from lignocellulosic biomass is recognized as a promising route to produce biobased fuels responsibly [1]. Lignocellulosic biomass consists of cellulose, lignin and hemicellulose. Bioconversion into valuable products is done in two steps: the hydrolysis of polysacharides into monosacharides and the subsequent fermentation into the product of interest. Unfortunately the harsh conditions used during the pretreatment of the biomass produce toxins that inhibit fermentation and consequently need to be removed [2]. The main classes of toxins are identified as acids, phenols and furans [1]. This study focusses on the removal of one of these classes, i.e. furans from glucose product streams. In earlier work, styrene based resins have been identified as promising adsorbents for the separation of furans from sugars [3-5]. Adsorption of furans is established by $\pi-\pi$ stacking of the furan ring with the styrene group of the resin. In previous work we investigated several styrene based resins, with and without anion exchange groups, for the removal of 5-hydroxymethylfurfural from glucose [3]. Dowex ${ }^{T M}$ Optipore $^{\mathrm{TM}}$ L493 (Optipore) was identified as the most promising adsorption resin for HMF adsorption over a broad HMF concentration range, based on equilibrium adsorption isotherms [3]. Table 1 summarizes the main properties of the Optipore resin. The excellent adsorption qualities are due to the resins high surface area $\left(>1100 \mathrm{~m}^{2} / \mathrm{g}\right)$ [3]. Furthermore, the apolar resin does not adsorb the polar glucose and sugar loss due to adsorption is therefore negligible, nor does the presence of glucose influences the adsorption of HMF.

Dowex classifies Optipore as an enhanced resin with well-connected pores [6]. This does not only affect the adsorption capacity but also the adsorption kinetics. Adsorption in the particle is achieved through diffusion through the pores or over the surface of the pores or by a combination of both. If the pores are not well connected, the diffusion path is long. In that case, long times are required to reach equilibrium and adsorption kinetics are slow. However, since the pores of Optipore are well connected, adsorption kinetics are assumed to be fast, as was also observed by Wegmann et al. [7] for the removal of acrylonitrile from water using Optipore. In addition to our previous study where we investigated static equilibrium adsorption behavior, we here investigate the adsorption 
kinetics of the adsorption of HMF in Optipore, which are essential to design a process for practical application. The effective diffusion coefficient of HMF in Optipore is studied by zero length column (ZLC) experiments and breakthrough experiments. The experimental values of the ZLC experiments are modelled with a homogeneous solid diffusion model to obtain the effective diffusion coefficient of HMF inside the particle and the external mass transfer coefficient $\left(\mathrm{k}_{\mathrm{f}}\right)$. Although other research has shown that a similar resin, Amberlite XAD4, shows good kinetic results for the removal of furan derivatives from water or a glucose solution $[4,8]$, it is expected that Optipore, due to its enhanced and well-connected pores will show even superior results [6]. All experiments are conducted with HMF in water and HMF in aqueous glucose $(100 \mathrm{~g} / \mathrm{l})$. Since previous research [3] showed that the presence of glucose does not affect HMF adsorption, it is expected that glucose only influences the HMF adsorption kinetics by increasing the viscosity of the solution.

Table 1: Properties of Dowex Optipore L493 [6].

Matrix structure

Macroporous styrenic polymer

Crosslinker $[9,10]$

Divinylbenzene

Particle diameter [3]

$557 \mu \mathrm{m}$

BET surface area

$>1100 \mathrm{~m}^{2} / \mathrm{g}$

Average pore diameter

$46 \AA$

Apparent density

$0.62 \cdot 10^{6} \mathrm{~g} / \mathrm{m}^{3}$

Total porosity

$1.16 \cdot 10^{-6} \mathrm{~m}^{3} / \mathrm{g}$

\subsection{Homogeneous solid diffusion model (HSDM)}

Adsorption of a solute on a macroporous adsorbent consists of several steps. First the solute diffuses from the bulk solution to the boundary layer formed by the stagnant layer surrounding the 
adsorbent. Then it diffuses through the boundary layer to the surface of the particle, from where it diffuses into and through the particle. The diffusion into a macroporous adsorbent is usually through the pores of the particle (pore diffusion) [11], over the adsorption sites on the pore walls (surface diffusion) [12] or by a combination of both [13].

For the determination of the diffusion coefficient, a zero length column is used [14]. In this experiment the adsorbent material is fixed in a column with a very short bed length and a solution is circulated over the column. Due to the short bed length, the concentration difference between the inflow and outflow of the column is very small and the system approaches an ideally mixed finite bed. When the concentration of the solution is monitored in time, the effective diffusion coefficient can be determined by fitting the experimental data to a kinetic model.

While the exact adsorption mechanism in an adsorbent is a complex interaction between the diffusion in the external film, the pore, the surface and bulk material and sorbate-sorbent interaction, it is usually modelled with simplified models [15]. Those models regard the adsorbent as a two component (macropororous) system with diffusion through the pores or over the pore surfaces (heterogeneous) or the adsorbent is considered as a bulk material (homogeneous) with diffusion in the bulk phase. Additionally, also a combination of both is possible [16]. The homogeneous solid diffusion model (HSDM) is frequently used to describe adsorption kinetics of solutes on adsorbents. This model assumes that molecules are first adsorbed at the external surface of the resin bead, and then diffuse further into the particle as a result of surface concentration gradients [12]. In the HSDM, diffusion in a resin is assumed as diffusion in a homogeneous solid particle. The diffusion coefficient is then calculated based on the particle diameter. The actually diffused path length through the pore, determined by the pore tortuosity is not taken in account. In the case of macroporous resins, were adsorption is mainly through the pores or over the pore surfaces, the obtained effective diffusion constant gives the practical bulk diffusion coefficient of the resin, but does not provide further insight in the specific diffusion coefficient of the material. However for gel type resins the obtained diffusion coefficient does give proper insight in the kinetic properties of the material. There are two reasons why we chose the 
HSDM to model the bulk diffusion coefficient. First, the specific particle parameters, such as tortuosity, that are required for proper modeling using other models are not well known. Second, although Optipore is classified as a macroporous resin that contains small pores [6], literature on hypercrosslinked resins states that the porous structure is different from common heterogeneous macroporous resins and that is is rather regarded as a homogeneous single phase polymeric gel [17]. For homogeneous materials, such as gel type materials, the HSDM very well describes the mass transfer mechanism [16].

The kinetic HSDM model that describes adsorption in a column is based on the mass balance of the adsorbate. The following is assumed [12]:

- There is no concentration gradient over the bed. This is achieved by using a zero length column in which the bed is very short ( 2 $\mathrm{mm})$;

- The adsorbent is spherical with uniformly distributed pores.

- Equilibrium is described by Freundlich as was determined earlier [3];

- The diffusion is described by Fick's law. The concentration adsorbent is very low and therefore Ficks law can be assumed;

For these conditions the following equations can be solved in order to obtain the effective surface diffusion coefficient, the liquid film mass transfer coefficient and subsequently, the Biot number [12].

The differential mass balance of the HSDM of a solute in a spherical particle is given by (Equation 1):

$\frac{\partial q}{\partial t}=D_{\text {eff }}\left(\frac{\partial^{2} q}{\partial r^{2}}+\frac{2}{r} \frac{\partial q}{\partial r}\right)$

Were $q$ is the HMF adsorption capacity of the resin $\left(g_{\text {solute }} / g_{\text {resin }}\right)$, $t$ is the time $(s)$ and $r$ is the radial position within the particle $(\mathrm{m})$ with an origin at the center of the particle. $D_{\text {eff }}$ is the effective surface diffusion coefficient $\left(\mathrm{m}^{2} / \mathrm{s}\right)$ and is a measure for how fast the molecules diffuse inside the particle. 
The adsorption equilibrium between solute and adsorbent is described by Freundlich [3] (Equation 2) :

$$
q=K \cdot c_{e q}^{1 / n}
$$

$\mathrm{q}\left(\mathrm{g}_{\text {solute }} / \mathrm{g}_{\text {resin }}\right)$ is the adsorption capacity at equilibrium concentration $\mathrm{c}_{\text {eq }}\left(\mathrm{g}_{\text {solute }} / \mathrm{l}\right), \mathrm{K}$ is the Freundlich equilibrium constant $\left(1 / \mathrm{g}_{\mathrm{resin}}\right)$.

Equation 1 is solved assuming the following boundary condition (Equation 3) :

$$
\frac{\partial q(r=0, t)}{\partial r}=0
$$

Signifying that there is no flux across the center of the particle.

When a stagnant film (liquid film boundary layer) is present around the particle and the effective diffusion inside the particle is fast compared to the mass transfer through this stagnant layer the following boundary condition is also necessary:

$\rho_{p} D_{\text {eff }} \frac{\partial q\left(r=r_{p, t}\right)}{\partial r}=k_{f}\left(c_{b}-c_{s}\right)$

Were $\rho_{p}$ is the apparent particle density $(g / l), c_{b}, c_{s}$ are the bulk and solid-liquid interface concentration respectively $\left(g_{\text {solute }} / l\right)$. In this equation the kinetic parameter, $k_{f}$ is introduced. This is the liquid film boundary layer mass transfer coefficient $(\mathrm{m} / \mathrm{s})$ and is a measure for how fast the molecules diffuse across the stagnant liquid film boundary layer around the particles. Equation 4 states that the flux of the solute from the bulk through the liquid film boundary layer around the particle to the particle itself is equal to the flux from the surface of the particle to the center of the particle.

The initial conditions to solve Equation 1 are:

$$
\begin{aligned}
& c_{b}(t=0)=c_{0} \\
& q(r, t=0)=0
\end{aligned}
$$


Were $c_{0}$ is the bulk solute concentration $\left(g_{\text {solute }} /\right)$. The initial condtions of Equation 5 and 6 state that initially the bulk concentration is equal to the initial concentration and the particle is free of adsorbent.

This set of equations can be solved in Matlab with different combinations of the kinetic parameters $k_{f}$ and $D_{\text {eff }}$ to determine the best fit with the experimental data. $k_{f}$ and $D_{\text {eff }}$ can then be optimized by minimizing the sum of squares between the calculated $c_{b}$ and the measured $c_{b}$.

The Biot number $(\mathrm{Bi})$ is an indication of the ratio of the rate of transport through the liquid film boundary layer $\left(k_{f}\right)$ and the transport inside the particle $\left(D_{\text {eff }}\right)$. For $B i \ll 1$ the external mass transport is the rate limiting step while for $\mathrm{Bi}>>100$ the diffusion in the particle is the rate limiting step [12]. The Biot number is calculated by the following equation [12] (Equation 7):

$B i=\frac{k_{f} \cdot r_{p} \cdot c_{0}}{D_{\text {eff }} \cdot \rho_{p} \cdot q_{0}}$

With $k_{f}$ the liquid film mass transfer coefficient $(m / s), r_{p}(m)$ the particle radius, $c_{0}\left(g_{\text {solute }} /\right)$ the initial concentration, $D_{\text {eff }}\left(\mathrm{m}^{2} / \mathrm{s}\right)$ the effective diffusion coefficient of the solute in the particle $\rho_{p}(g / l)$ the particle density, including the open volume, and $q_{0}\left(g_{\text {solute }} / g_{\text {resin }}\right)$ the adsorption capacity in equilibrium with $\mathrm{c}_{0}$.

\subsection{Materials and Methods}

\subsubsection{Materials}

The resin Dowex Optipore L-493 (Optipore), 5-hydroxymethylfurfural (HMF), glucose and sulfuric acid $\left(\mathrm{H}_{2} \mathrm{SO}_{4}\right)$ were purchased from Sigma Aldrich. Impurities present in the resin were removed by rinsing with MilliQ water prior to use until impurities were no longer detectable in the rinse water by UV-VIS (200-400 nm).

\subsubsection{Solute analysis}

HMF in water was analysed with a UV-VIS detector at $284 \mathrm{~nm}$. When HMF was mixed with glucose, the HMF and glucose concentrations were determined with a Waters HPLC with a 515 
pump, a 2487 dual $\lambda$ adsorbance detector and Millenium ${ }^{32}$ (version 3.20) software. Analysis was carried out with a Resex ROA-organic acid $300 \times 7.8 \mathrm{~mm}$ column (Phenomenex). The mobile phase was $0.005 \mathrm{M} \mathrm{H}_{2} \mathrm{SO}_{4}$. The column operation temperature was $90^{\circ} \mathrm{C}$ and the mobile phase flow rate was $0.3 \mathrm{ml} / \mathrm{min}$. Peak detection was performed using UV at $284 \mathrm{~nm}$ (HMF) and $205 \mathrm{~nm}$ (glucose).

\subsubsection{Zero length column experiments}

The effective diffusion coefficient was determined with a zero length colum (ZLC) set up as depicted in Figure 1. The ZLC set up used an Akta system. This system was equipped with two pumps and a UV detector. The resin was packed in a column (Omnifit, $6.6 \mathrm{~mm}$ internal diameter) that was connected between the pump and the UV detector. The stream leaving the UV detector was recirculated to the feed container.

To conduct the ZLC experiments, the column was packed with the resin with a bed height of $2 \mathrm{~mm}$. The column was subsequently equilibrated with water by rinsing the entire system, including the packed column, with MilliQ water (10 times the entire system volume). After equilibration, the column was disconnected and the entire system, including the feed container and the tubes, was filled with the adsorption solution (5 mg/l HMF or $5 \mathrm{mg} / \mathrm{l} \mathrm{HMF}$ in $100 \mathrm{~g} / \mathrm{lg}$ glucose). The column was reconnected and the HMF solution was pumped through the column in recycle mode. The total volume of the system, including the feed container, was $17 \mathrm{ml}$. When HMF was adsorbed from the water, the HMF concentration was monitored in time using the UV detector connected to the Akta. When a HMF/glucose solution was used as a feed, analysis was performed by taking small samples from the feed container $(0.05 \mathrm{ml})$ that were analyzed using HPLC. In the first 2 minutes, samples were taken every minute, then for 15 minutes every 5 minutes and then for half an hour every 15 minutes, followed by samples taken every half hour till the end of the experiment. The ZLC experiments were conducted at a flow rate of $10 \mathrm{ml} / \mathrm{min}$ and $20 \mathrm{ml} / \mathrm{min}$ for HMF from water and at $20 \mathrm{ml} / \mathrm{min}$ for HMF from aqueous glucose. 


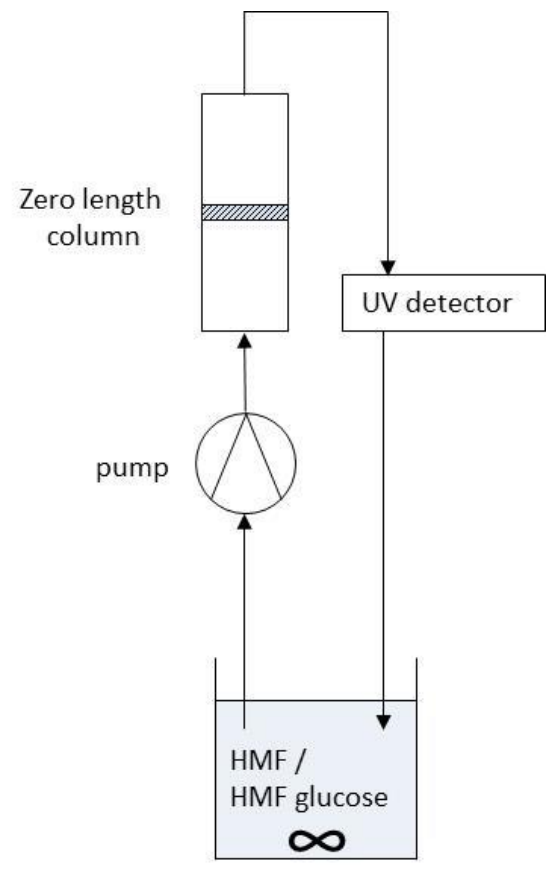

Figure 1: Schematic representation of experimental zero length column (ZLC) set up.

\subsubsection{Breakthrough experiments}

\section{Adsorption}

The breakthrough curves for HMF adsorption were determined with a set up shown in Figure 2. Optipore resin was packed in an Omnifit column (length: $100 \mathrm{~mm}$, diameter: $6.6 \mathrm{~mm}$ or length: 450 $\mathrm{mm}$, diameter: $20 \mathrm{~mm}$ ). The experiments were consequently performed with a bed volume (BV) of 5 or $50 \mathrm{ml}$. The reason for this difference was practical column availability and number of BV necessary for completion of the breakthrough curve. The number of BV necessary for the determination of a low concentration $(5 \mathrm{mg} / \mathrm{l})$ breakthrough curve is very high, so we use a small column and therefore small BV. Validation experiments to see if the results from both columns were comparable were performed. The breakthrough curves were determined at a flow rate of 8 bed volumes per hour (BV/h) with HMF (5 mg/l and $5000 \mathrm{mg} / \mathrm{l})$ from water and HMF (5000 mg/l) from $100 \mathrm{~g} / \mathrm{l}$ aqueous glucose. The effect of the flow rate on the column performance was evaluated by varying the flow rate $(4,8$ and $12 \mathrm{BV} / \mathrm{h})$ for HMF from water. All experiments were 
conducted at $20{ }^{\circ} \mathrm{C}$. The outflow of the column was fractionated in fractions with a volume of 0.5 BVs. The concentration of every other fraction was analyzed with UV-VIS (HMF from water) or with HPLC with a UV detector (HMF from glucose).

The HMF column capacity (\%) was determined at $100 \%$ loading of the column and is expressed as the percentage of the equilibrium adsorption capacity as determined earlier [3]. The HMF column adsorption efficiency (\%) was determined at $10 \%$ breakthrough of the inlet concentration and is expressed as percentage of the column capacity.

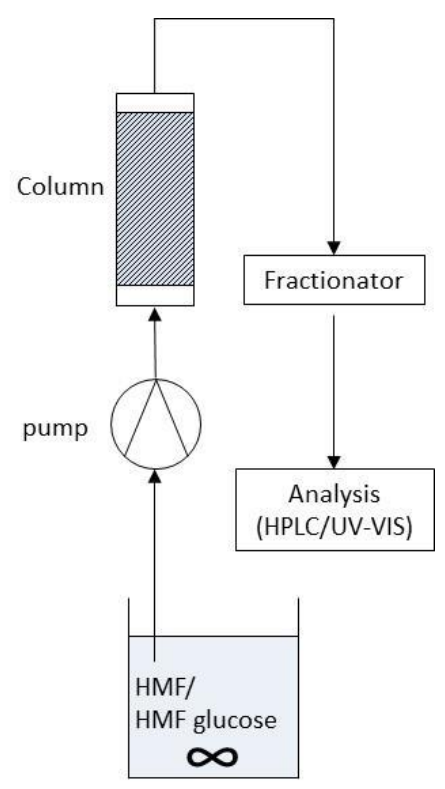

Figure 2: Schematic representation of the breakthrough set-up.

\section{Desorption}

Desorption experiments were performed after the column was fully loaded with $5000 \mathrm{mg} / \mathrm{HMF}$. For this experiment the same set up was used as for the breakthrough curves (Figure 2). Water was pumped through the pre-loaded column with $8 \mathrm{BV} / \mathrm{h}$ at $20^{\circ} \mathrm{C}$ and $60^{\circ} \mathrm{C}$. The outflow of the column was fractionated in fractions with a volume of $0.5 \mathrm{BV}$. The concentration of every other fraction was analyzed with UV-VIS (HMF from water) or with HPLC with a UV detector (HMF from glucose). 


\subsection{Results and Discussion}

\subsubsection{Zero length column experiments}

The effective diffusion coefficient of HMF in Optipore is determined from ZLC experiments, both for HMF adsorption from water and from an aqueous glucose solution. Figure 3 shows the HMF concentration in time (normalized for the initial HMF concentration at $t=0$ ) for both solutions. The experimental data were modelled with the HSDM to determine $D_{\text {eff }}$ and $k_{f}$ (Table 2).

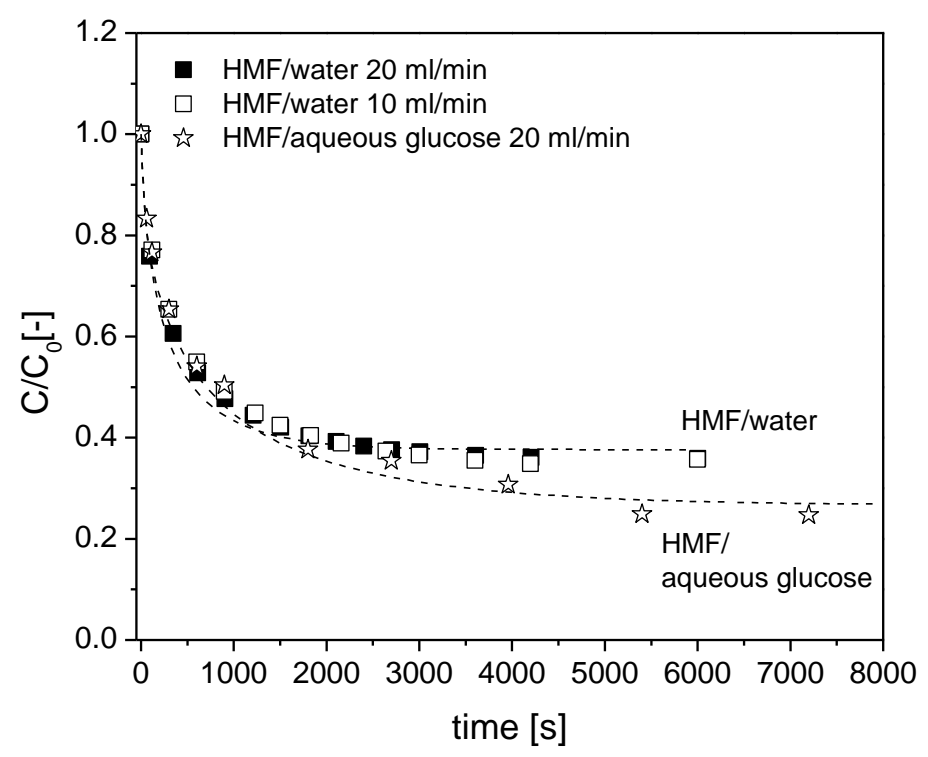

Figure 3: HMF concentration normalized for initial HMF concentration $\left(\mathrm{c}_{0}\right)$ in time (symbols) and relative HMF concentration calculated by HSDM (lines) [12]. 
Table 2: Effective diffusion coefficients $\left(\mathrm{D}_{\text {eff }}\right)$, kinetic parameter $\left(\mathrm{k}_{\mathrm{f}}\right)$ and Biot number $(\mathrm{Bi})$ of HMF adsorption in Optipore.

Water

$10 \mathrm{ml} / \mathrm{min}$

HSDM

$\begin{array}{lccc}\mathrm{D}_{\text {eff }}\left(\mathrm{m}^{2} / \mathrm{s}\right) & 8.0 \cdot 10^{-12} & 8.3 \cdot 10^{-12} & 3.0 \cdot 10^{-12} \\ \mathrm{k}_{\mathrm{f}}(\mathrm{m} / \mathrm{s}) & >8 \cdot 10^{-5} & >8 \cdot 10^{-5} & 7 \cdot 10^{-5} \\ \mathrm{Bi}(-) & >13 & >11 & 26\end{array}$

Theroretical

correlations

$[18,19]$
$D_{A B}{ }^{\circ}\left(m^{2} / s\right)$
$1.1 \cdot 10^{-9}$
$1.110^{-9}$
$0.7 \cdot 10^{-9}$
$\mathrm{k}_{\mathrm{f}, \mathrm{th}}(\mathrm{m} / \mathrm{s})$
$6 \cdot 10^{-5}$
$8 \cdot 10^{-5}$
$6 \cdot 10^{-5}$

Aqueous glucose

$20 \mathrm{ml} / \mathrm{min}$

$20 \mathrm{ml} / \mathrm{min}$

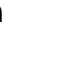


solutions with $k_{f}>8 \cdot 10^{-5} \mathrm{~m} / \mathrm{s}$ give a good fit for the experimental data. Although $k_{f}$ is higher than $810^{-5} \mathrm{~m} / \mathrm{s}$, its exact value cannot be determined from these data, as the adsorption kinetics are no longer sensitive to $k_{f}$ at these flow rates and this $D_{\text {eff }}$ value. This shows that the external mass transfer indeed does not limit the adsorption, as was already observed from the experimental data. The effective diffusion coefficient in the particle is, as expected, equal for both flow rates, because the diffusion coefficient inside the particle is independent of the flow rate outside the particle.

The theoretical diffusion coefficient of HMF in stagnant water $\left(D_{A B}{ }^{0}\right)$ estimated with the WilkeChang correlation [18] is $1.110^{-9} \mathrm{~m}^{2} / \mathrm{s}$ (Table 2). The coefficient is reduced by a factor $\sim 10^{2}$ when HMF diffuses into the particles. This is due to hindrance of the HMF diffusion by the walls of the pores as well as by adsorption to the walls of the pores [21].

Equilibrium of HMF adsorption from aqueous glucose is reached later, at $\sim 5000$ s. This is caused by several reasons. When glucose is added to the water, the viscosity increases, reducing the mixing around the particle, thereby increasing the stagnant film layer thickness around the particle. Also the higher viscosity reduces the diffusion through the thicker stagnant film layer as is also indicated by the increased $D_{A B}{ }^{0}$ (the by Wilke-Chang correlated diffusion coefficient of HMF in a stagnant liquid) (Table 2). This is also observed from the theoretically determined value of the external mass transport factor $\left(\mathrm{k}_{\mathrm{f}, \mathrm{th}}\right)$, which is calculated with the Gnielinski correlation [19]; it is lower for a glucose solution (Table 2). The data obtained from the modelling of our experimental data confirm this: $D_{\text {eff }}$ also shows that the diffusion inside the particle is reduced in the presence of glucose. Table 2 shows that the effective diffusion coefficient determined with the HSDM of HMF in glucose is approximately three times lower than when HMF is adsorbed from water. In previous research [3] we measured that glucose does not adsorb on the styrene matrix of Optipore. Furthermore, we found that glucose does not alter the solubility of HMF and that the adsorption capacity of Optipore for HMF remains constant independent of the presence of glucose. This suggested that glucose does not have any thermodynamic effect on HMF diffusion (weaker or stronger binding to the resin). The reduced $D_{\text {eff }}$ indicates that HMF diffuses into the particle, probably not only by surface diffusion over the walls of the pores, as is the case in small pores of microporous materials, but probably also by pore diffusion when the HMF is out of the range of the attractive force field of the pore surface, which is possible in the larger pores of macroporous 
materials [22]. Glucose also increases the viscosity of the solution in the pores and this would explain the lower effective diffusion coefficient in the particle.

In the presence of glucose the HSDM model only fits with the experimental data when $k_{f}$ is $7 \cdot 10^{-5}$ $\mathrm{m} / \mathrm{s}$, indicating that the process is, next to restriction from the internal mass transport, also limited by external mass transport. This is also indicated by the Biot number, which is 26 , indicating that the process is limited by internal as well as external mass transport.

\subsubsection{Breakthrough experiments}

The breakthrough curve of HMF on an Optipore packed column was investigated because the breakthrough time and the slope of the breakthrough curve indicate the effectiveness of the adsorption at the chosen conditions.

\section{Breakthrough curve at low HMF concentration}

The breakthrough curve of $5 \mathrm{mg} / \mathrm{l} \mathrm{HMF}$ in water was determined with a column with a volume of 5 $\mathrm{ml}$. The breakthrough curve is shown in Figure 4. The breakthrough curve was only measured once. We believe this is allowed since validation experiments performed later (Figure 5) show that the breakthrough curves are reproducible. 


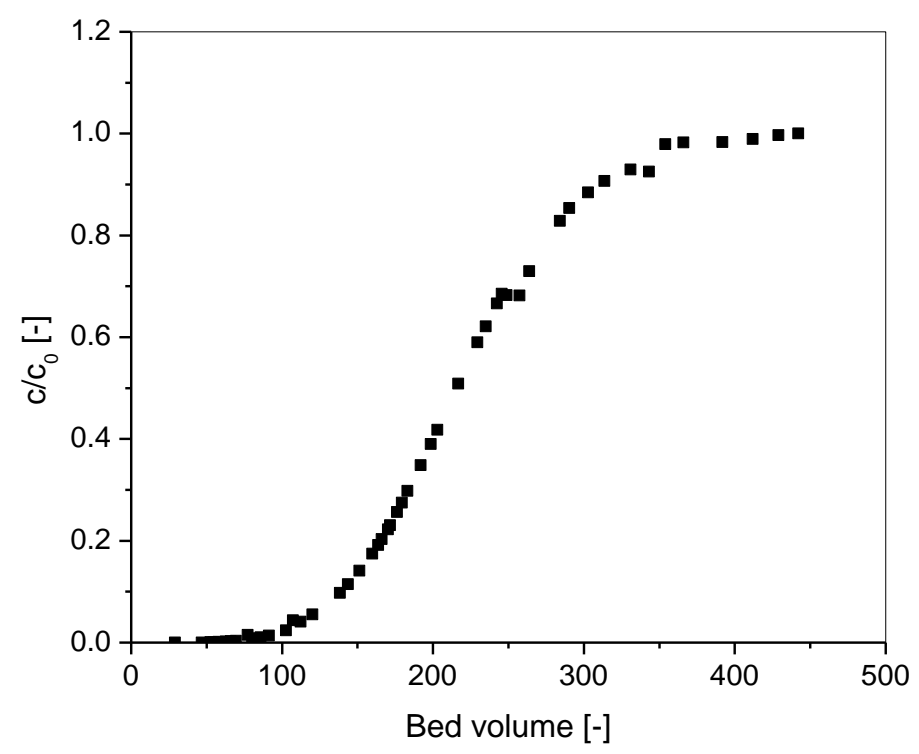

Figure 4: HMF breakthrough curve at $20^{\circ} \mathrm{C}, 5 \mathrm{mg} / \mathrm{l} \mathrm{HMF}$ in water, BV: $5 \mathrm{ml}, 8 \mathrm{BV} / \mathrm{h}$.

Figure 4 shows that breakthrough starts at $\sim 95 \mathrm{BV}$ and is complete after $\sim 400 \mathrm{BV}$. The column adsorption capacity is determined by measuring the adsorbed HMF when the column is completely loaded. The adsorption capacity of the resins in the packed bed is $1.4 \mathrm{mg} / \mathrm{g}$, this is $99 \%$ of the equilibrium adsorption capacity of the resins as determined from the adsorption isotherm in earlier work [3]. Consequently packing of the resins in a packed bed does not change the adsorption capacity as the equilibrium capacity is similar to that earlier determined in batch experiments. The column adsorption efficiency at $10 \%$ breakthrough is $80 \%$ of the column adsorption capacity. Hand et al. [23] defined three categories of breakthrough curves depending on the Biot number, $\mathrm{For} \mathrm{Bi}<$ 0.5 , the process is controlled by the liquid-phase mass transfer rate, for $0.5<\mathrm{Bi}<30$ the process is controlled by liquid-phase and solid-phase mass transfer, while for $\mathrm{Bi}>30$, the solid phase mass transfer rate controls the adsorption process. The calculated Bi number at $5 \mathrm{mg} / \mathrm{l}$ is 1.67 which makes the process dependent on both liquid and solid phase mass transfer. In the ZLC experiments the process was limited by internal diffusion only, in a packed bed the operating flow rate is much lower resulting in a process that is also controlled by external transport.

At these operating conditions (e.g. flow rate, HMF concentration, residence time), the adsorption capacity of the bed is very effective and breakthrough only occurs after treatment of $\sim 100 \mathrm{BV}$. These results make the resin Optipore a very promising candidate for HMF removal. 
Due to the long experimental time and the high number of BV needed to determine the breakthrough curves at this concentration, the other breakthrough curves, investigating the effect of flow rate and the presence of glucose, were determined at a higher HMF concentration ( $5000 \mathrm{~g} / \mathrm{l})$ to decrease the experimental time and the number of BV treated.

Investigation breakthrough curves at high HMF concentration

The experimental time and number of treated BV is reduced by increasing the HMF concentration (5000 mg/l) during the breakthrough experiments (Figure 5). This breakthrough curve is shown twice determined with a BV of $50 \mathrm{ml}$ to show the reproducibility of the experiments and once with a BV of $5 \mathrm{ml}$ to show that the data obtained with a column of $5 \mathrm{ml}$ and $50 \mathrm{ml}$ are comparable.

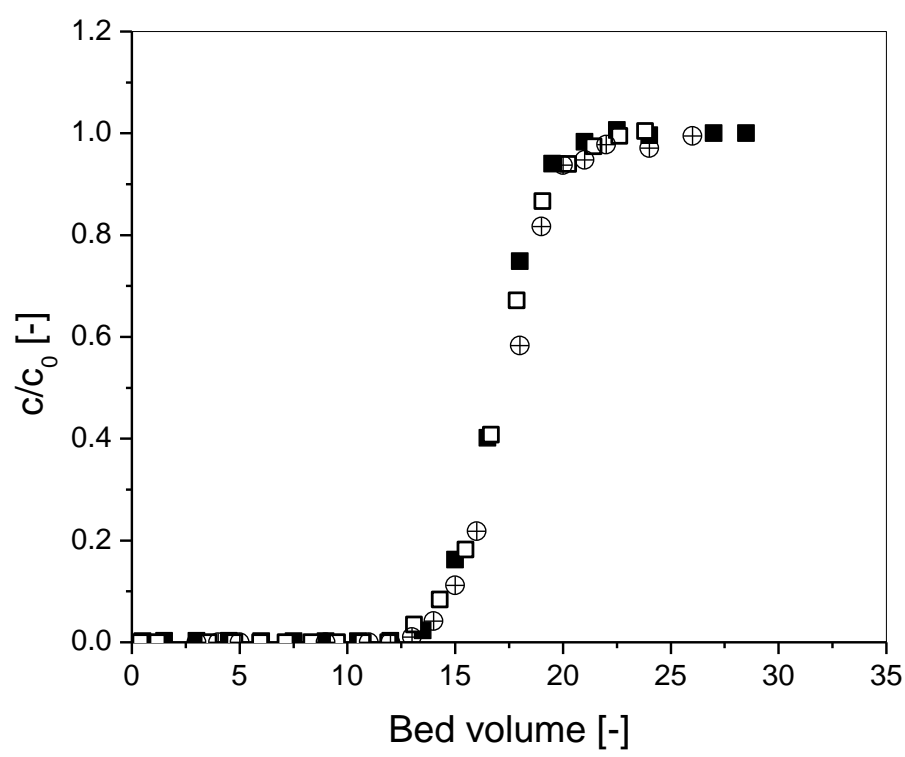

Figure 5: HMF breakthrough curves $20^{\circ} \mathrm{C}, 5000 \mathrm{mg} / \mathrm{HMF}$ in water, $8 \mathrm{BV} / \mathrm{h}$ : a experiment 1 (BV: $50 \mathrm{ml}$ ); $\square$ experiment 2 (BV: $50 \mathrm{ml}) ; \oplus$ experiment $3(\mathrm{BV}: 5 \mathrm{ml})$.

Figure 5 shows that the two curves determined with a $50 \mathrm{ml}$ column coincide and that the reproducibility of the measurements is very good. This reproducibility is representative for all other breakthrough experiments. The curve obtained with the $5 \mathrm{ml}$ column also coincides with the other curves, showing that the data obtained with the two columns are comparable. 
Breakthrough starts at $\sim 13 \mathrm{BV}$ and is completed at $\sim 23 \mathrm{BV}$. The curve is steep and has a regular S-shape indicating efficient HMF adsorption. This is also reflected by the column efficiency of $87 \%$ at $10 \%$ breakthrough. A steep slope of the breakthrough curve indicates that the mass transfer zone is small and that axial diffusion through the bed is negligible.

The column adsorption capacity at $100 \%$ breakthrough is only $52 \%$ of what was found in earlier batch experiments [3] and much lower than the column adsorption capacity achieved with $5 \mathrm{mg} / \mathrm{l}$. This is unexpected and not understood. Usually packed beds (especially at higher concentrations) establish higher adsorption capacities compared to batch experiments, due to their higher concentration gradient and therefore larger driving force at the adsorption zone, while batch experiments experience a decreasing concentration gradient in time reducing the driving force [24, 25].

Effect of flow rate

Figure 6 shows the HMF breakthrough curves for 4,8 and $12 \mathrm{BV} / \mathrm{h}$.

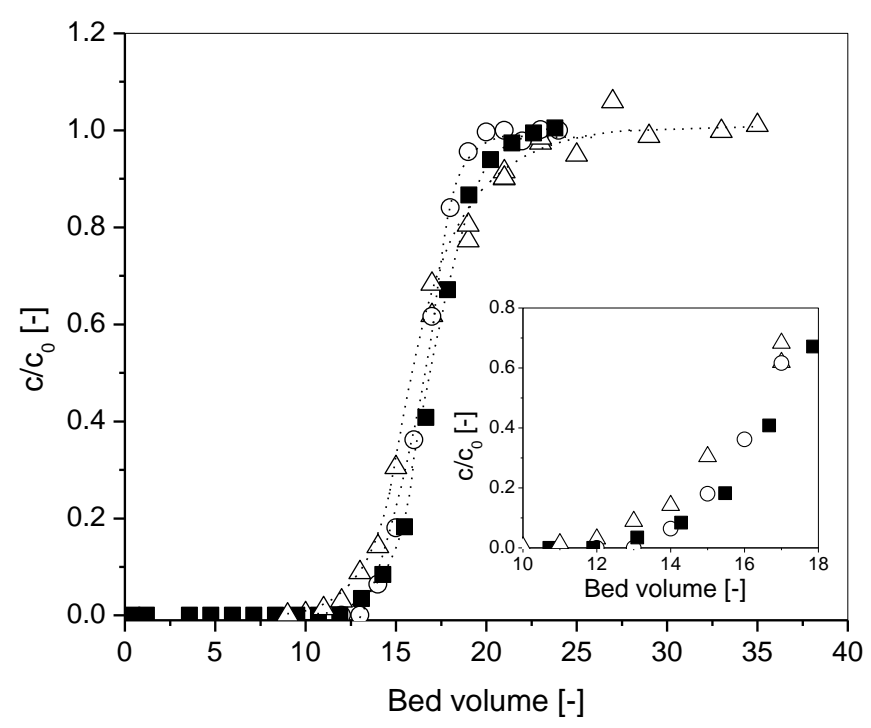

Figure 6: $\operatorname{HMF}(5 \mathrm{~g} / \mathrm{l})$ breakthrough curves at different flow rates at $20{ }^{\circ} \mathrm{C}, \mathrm{BV}: 5 \mathrm{ml}: \circ 4 \mathrm{BV} / \mathrm{h} ; \mathbf{- 8 B V} / \mathrm{h} ; \Delta 12 \mathrm{BV} / \mathrm{h}$. 
Figure 6 shows that the breakthrough is at $\sim 12 \mathrm{BV}$ for $12 \mathrm{BV} / \mathrm{h}, \sim 13 \mathrm{BV}$ for $8 \mathrm{BV} / \mathrm{h}$ and $\sim 14$ for 4 $\mathrm{BV} / \mathrm{h}$. The curves show the lowest slope for $12 \mathrm{BV} / \mathrm{h}$ while the slope increases when the flow rate is reduced to 8 and $4 \mathrm{BV} / \mathrm{h}$. Both the slightly earlier breakthrough as well as a shallower slope and the tailing indicate a little less efficient adsorption. At low flow rates the residence time in the column is longer and HMF has more time to adsorb. Although this research shows that longer adsorption times affect the breakthrough time and slope, this effect is only marginal. The residence time shows only a small effect on the adsorption efficiency indicating that the internal adsorption is very efficient.

A much larger effect of the flow rate on the adsorption efficiency was seen with Amberlite XAD4, an adsorbent with a comparable chemical structure [26]. Gupta et al. [8] investigated the effect of flow rate on the breakthrough of $5000 \mathrm{mg} / \mathrm{l}$ furfural in a column packed with Amberlite XAD4. The breakthrough times of furfural on Amberlite XAD 4 are much shorter. At the selected flow rates of 5 , 7 and $12 \mathrm{BV} / \mathrm{h}$, the breakthrough times are 7.7, 6.9 and $4.5 \mathrm{BV}$ respectively. These much earlier breakthrough times are caused by two reasons. Optipore has a higher adsorption capacity than Amberlite XAD4 due to the much higher surface area [3]. Furthermore the large effect of the flow rate on the breakthrough times and the adsorption capacity, which are both reduced with increasing flow rates indicate that, in contrast to Optipore, at these conditions, adsorption in Amberlite XAD4 is controlled by internal particle diffusion.

Weil et al. [4] could only establish comparable breakthrough times ( 13 BV) with $5000 \mathrm{mg} / \mathrm{l}$ furfural on a column packed with Amberlite XAD4 by reducing the flow rate $\sim 6$ times to $2.3 \mathrm{BV} / \mathrm{h}$. And although the breakthrough time at these low flow rates was comparable to that of Optipore, the slope of the curve was very shallow, which is another indication that internal diffusion limits adsorption in Amberlite XAD4 at these conditions. So far Amberlite XAD4 has been identified as a good adsorber for furan derivate removal. This research shows that throughput and capacity are even better and improved enormously when Optipore is used.

Optipore is a hypercrosslinked polymer [17, 27]. These polymers are prepared by emulsion polymerization and are further crosslinked while swollen in a good solvent to increase the adsorption capacity. Previous research showed that due to this treatment the polymer also swells 
much more in water compared to resins with similar chemical structures [3, 28]. We assume that this swelling is also responsible for a good connectivity of the pores and therefore the higher solute diffusion in the particle.

\section{Effect aqueous glucose on HMF breakthrough}

ZLC experiments showed that the diffusion coefficient is reduced when HMF is adsorbed from an aqueous glucose solution compared to adsorption from pure water. Breakthrough experiments of HMF in aqueous glucose solutions were performed as well to examine the effect of the presence of glucose on the column adsorption efficiency. Figure 7 shows the breakthrough curves of both HMF and glucose from an HMF/aqueous glucose solution. The breakthrough curve of HMF from water is added as reference. The small dip in the glucose breakthrough curve at $15 \mathrm{BV}$ is probably due to an inaccurate correction for the presence of HMF during HPLC analysis.

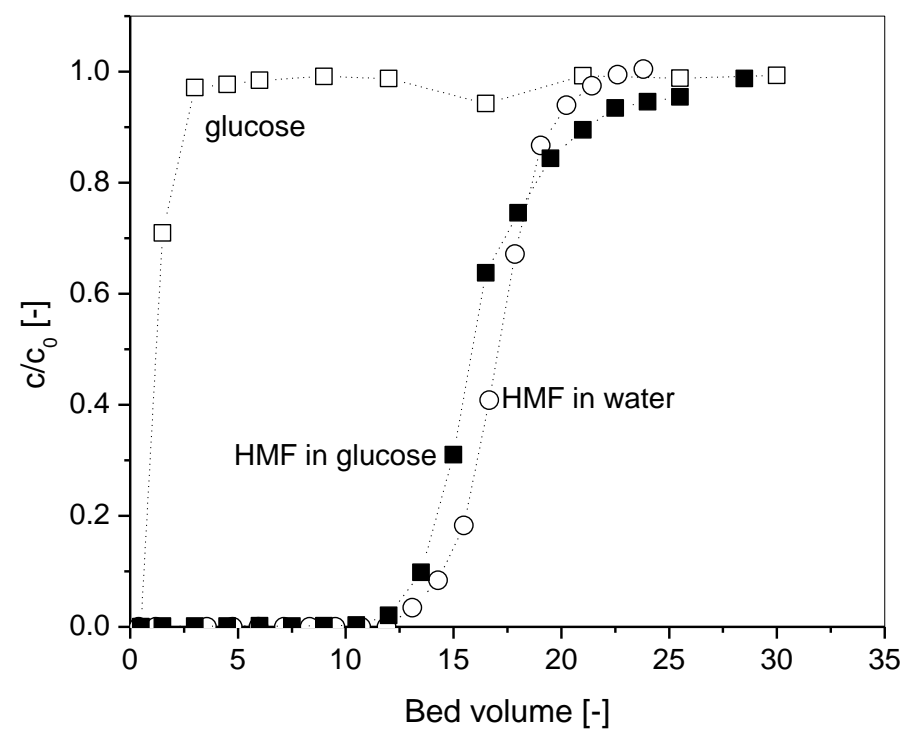

Figure 7: HMF (5 g/l) and glucose (100g/l) breakthrough curves at $20 \mathrm{C}$, BV: $50 \mathrm{ml}: \circ \mathrm{HMF}$ in water; a HMF from glucose; $\square$ glucose (from HMF aqueous glucose solution).

Glucose is detected at the end of the column after 1.5 BV and HMF breakthrough in aqueous glucose is observed at $12 \mathrm{BV}$. The fast breakthrough of glucose shows that glucose indeed does not adsorb on Optipore, as was shown as well earlier [3]. In the presence of glucose, an earlier 
breakthrough of HMF is observed than in the presence of pure water and also the slope of the curve is less steep and more tailing is observed. This is explained by the higher viscosity of the glucose solution. This affects the kinetics of adsorption in two ways. As in the ZLC experiments, a higher viscosity increases the stagnant film layer, thereby increasing the resistance to mass transport towards the particle. This is also seen in the correlated $k_{f, t h}$ numbers; When glucose is present, the $\mathrm{k}_{\mathrm{f}, \mathrm{th}}$ decreases from $1.65 \cdot 10^{-5} \mathrm{~m} / \mathrm{s}$ to $1.19 \cdot 10^{-9} \mathrm{~m} / \mathrm{s}$ indicating a higher resistance to mass transfer from the bulk solution to the particle. Secondly, ZLC experiments showed that the effective diffusion coefficient is also reduced by the presence of glucose (Table 2).

Weil et al. [4] performed breakthrough experiments on Amberlite XAD4 with furfural in water and furfural from aqueous glucose. The breakthrough curve (breakthrough time and slope of the curve) was unaffected by the presence of glucose in that case. However the concentration of glucose was 20 times lower $(5 \mathrm{~g} / \mathrm{l})$ than in our experiments and therefore the viscosity was almost similar to that of water. This support the hypothesis that the faster breakthrough and reduced slope we observe when glucose is present is caused by the viscosity increase of the solution and not by HMFglucose interactions.

\subsubsection{Desorption}

Desorption of HMF and HMF and glucose from the column was performed with water at $20{ }^{\circ} \mathrm{C}$ and $60{ }^{\circ} \mathrm{C}$ (Figure 8). The outlet concentration is normalized based on the feed concentration when the column was loaded. 


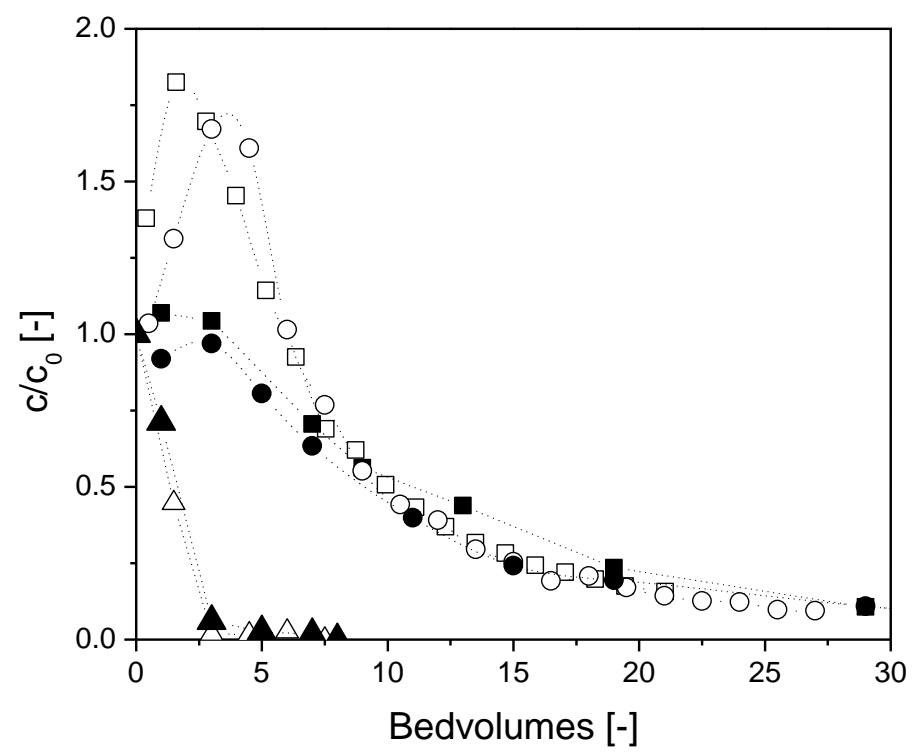

Figure 8: HMF and glucose desorption of the column with water, BV: $50 \mathrm{ml}$ : $\boldsymbol{\Delta}$ glucose desorption $20{ }^{\circ} \mathrm{C} ; \Delta$ glucose desorption $60^{\circ} \mathrm{C}$; $\bullet \mathrm{HMF}$ desorption with glucose $20^{\circ} \mathrm{C}$; $\square \mathrm{HMF}$ desorption without glucose $60^{\circ} \mathrm{C}$; ० $\mathrm{HMF}$ desorption with glucose $60^{\circ} \mathrm{C}$.

Figure 8 shows that glucose is released from the column almost immediately, hardly any glucose is present in the permeate after $3 \mathrm{BV}$ only. The $100 \mathrm{~g} / \mathrm{l}$ aqueous glucose solution has a viscosity of $1.3 \mathrm{MPa} \cdot \mathrm{s}$ at $20^{\circ} \mathrm{C}$ and of $0.6 \mathrm{mPa} \cdot \mathrm{s}$ at $60^{\circ} \mathrm{C}$ [29]. A bit more glucose is released from the column at $60{ }^{\circ} \mathrm{C}$, due to the reduced viscosity of the solution at this temperature, but this effect is rather small. Glucose release from the column is so fast because glucose is not adsorbed to the column but only present in the void volume of the column and pores of the adsorbent Optipore [3].

Figure 8 also shows the outlet concentration of HMF (without and in the presence of glucose) from the column at $20{ }^{\circ} \mathrm{C}$ and at $60{ }^{\circ} \mathrm{C}$. The relative concentration of released HMF reaches a value higher than 1 because the capacity of Optipore is much lower at $60{ }^{\circ} \mathrm{C}$ than at the loading temperature of $20{ }^{\circ} \mathrm{C}$ [3]. The percentage desorption expressed as percentage of the loading is given in Table 3. In the first $10 \mathrm{BV} \sim 1.5$ times more HMF is released from the column rinsed with water at $60{ }^{\circ} \mathrm{C}$ compared to the column rinsed with water at $20^{\circ} \mathrm{C}$. From $10 \mathrm{BV}$ onwards the amount of HMF desorbing from the column is equal for both temperatures. Still the desorption at 60 ${ }^{\circ} \mathrm{C}$ is much more efficient, as the amount of HMF still adsorbed to the column at that temperature is less than the still adsorbed to the column at $20^{\circ} \mathrm{C}$. The total amount of HMF desorbed after $30 \mathrm{BV}$ 
is $81 \%$ at $20{ }^{\circ} \mathrm{C}$ and $94 \%$ at $60{ }^{\circ} \mathrm{C}$. A better release of $\mathrm{HMF}$ at higher temperature was expected since the capacity of Optipore for HMF is lower at higher temperature [3].

Desorption of HMF is a bit faster when glucose is not present (Figure 8, Table 3). HMF desorption is lower when glucose is still present in the permeate (up to $\sim 3 \mathrm{BV}$ ). This is due to the increased viscosity of the glucose solution. As discussed earlier, this lowers the diffusion rate in the particle as well as the diffusion rate outside the particle in the stagnant boundary film layer.

Although HMF is released from the column by rinsing it with water, desorption requires a high amount of water. Other authors studied the desorption using different solvents (e.g. methanol, ethanol, n-propanol and n-butanol, acetone) [4, 30]. Although these solvents removed HMF effectively, their strong interaction with the resin, thus introducing another component in the system, is a major drawback. Increased desorption of HMF at higher temperature indicates that desorption with steam could offer a solution for HMF removal. 
Table 3: Percentage HMF desorbed after 10 and $30 \mathrm{BV}$ at $20^{\circ} \mathrm{C}$ and $60^{\circ} \mathrm{C}$, without and with glucose as percentage loading of the column.

Desorption after $10 \mathrm{BV}(\%)$

Desorption after 30 BV (\%)

$20{ }^{\circ} \mathrm{C}$

50

46

70

67

90

\subsection{Conclusion}

This study shows that, compared to often used resins, Optipore is a very efficient resin for HMF removal. Its fast kinetics and high capacity make it possible to efficiently remove HMF from glucose solutions.

The diffusion coefficients of HMF (5 mg/l) were $\sim 810^{-12} \mathrm{~m}^{2} / \mathrm{s}$ in water and $3.010^{-12} \mathrm{~m}^{2} / \mathrm{s}$ in a glucose $(100 \mathrm{~g} / \mathrm{l})$ solution. The reduced diffusion coefficient in the particle when glucose is present is caused by the higher viscosity of the glucose solution and indicates that diffusion is not only at the surface of the pore but that pore diffusion also plays a role in HMF adsorption.

The breakthrough curves of HMF on Optipore showed that the column is very efficient at the conditions tested. At $5 \mathrm{mg} / \mathrm{l}$, the adsorption is dependent on internal as well as external mass transport parameters. At $5000 \mathrm{mg} / \mathrm{l}$ the breakthrough curves showed external transport limitations. The desorption of HMF using water is improved when the temperature is increased, which would make desorption with steam an option for desorption. The results clearly show that at the operating conditions investigated (e.g. flow rate, HMF concentration, residence time), the adsorption capacity and kinetics of the resin Optipore are very effective, making it a very promising candidate for HMF removal. 


\subsection{Acknowledgment}

This work was carried out within project SC-00-04 of the Institute for Sustainable Process Technology (ISPT), The Netherlands. The authors would like to thank Christine Wegmann for her help in setting up the ZLC set up and the use of the Matlab code for the HSDM.

\subsection{References}

1. Mills, T.Y., N.R. Sandoval, and R.T. Gill, Cellulosic hydrolysate toxicity and tolerance mechanisms in Escherichia coli. Biotechnology for Biofuels, 2009. 2.

2. Huang, H.J., et al., A review of separation technologies in current and future biorefineries. Separation and Purification Technology, 2008. 62(1): p. 1-21.

3. IJzer, A.C., et al., Performance analysis of aromatic adsorptive resins for the effective removal of furan derivatives from glucose. Journal of Chemical Engineering and Biotechnology, 2014.

4. Weil, J.R., et al., Removal of fermentation inhibitors formed during pretreatment of biomass by polymeric adsorbents. Industrial \& Engineering Chemistry Research, 2002. 41(24): p. 6132-6138.

5. Fargues, C., R. Lewandowski, and M.-L. Lameloise, Evaluation of ion-exchange and adsorbent resins for the detoxification of beet distillery effluents. Industrial \& Engineering Chemistry Research, 2010. 49(19): p. 9248-9257.

6. Dow, Product information Dowex Optipore L493 and V493. Product information Dowex Optipore L493 and V493.

7. Wegmann, C., E. Suárez García, and P.J.A.M. Kerkhof, Kinetics of acrylonitrile adsorption from an aqueous solution using Dowex Optipore L-493. Separation and Purification Technology, 2011. 81(3): p. 429-434.

8. Gupta, P., et al., The removal of furfural from water by adsorption with polymeric resins. Separation Science and Technology, 2001. 36(13): p. 2835-2844.

9. Ramaswamy, S., H.-J. Huang, and B. Ramarao, in Separation and Purification Technologies in Biorefineries. 2013, John Wiley \& Sons, Ltd. p. 1-36.

10. Vern, C., et al., The beet sugar factory of the future. International Sugar Journal, 1995. 97(1159): p. 310-316.

11. Weber, W.J. and E.H. Smith, Simulation and design models for adsorption processes. Environmental Science \& Technology, 1987. 21(11): p. 1040-1050.

12. Traegner, U. and M. Suidan, Evaluation of surface and film diffusion coefficients for carbon adsorption. Water Research, 1989. 23(3): p. 267-273.

13. Susu, A.A., Mathematical modelling of fixed bed adsorption of aromatics and sulphur compounds in kerosene deodorisation. Chemical Engineering and Processing: Process Intensification, 2000. 39(6): p. 485-497.

14. Eic, M. and D.M. Ruthven, $A$ new experimental technique for measurement of intracrystalline diffusivity. Zeolites, 1988. 8(1): p. 40-45.

15. Hui, C.W., B. Chen, and G. McKay, Pore-surface diffusion model for batch adsorption processes. Langmuir, 2003. 19(10): p. 4188-4196.

16. Górka, A., et al., Ion exchange kinetics in removal of small ions. Effect of salt concentration on inter- and extraparticle diffusion. Chemical Engineering Science, 2008. 63(3): p. 637650 .

17. Tsyurupa, M.P. and V.A. Davankov, Porous stucture of hypercrosslinked polystyrene: State-of-the-art mini-review. Reactive and Functional Polymers, 2006. 66(7): p. 678-779.

18. Poling, B.E., J.M. Prausnitz, and J.P. O'Connell, The properties of gases and liquids. 2001: McGraw-Hill. 
19. Roberts, P., P. Cornel, and R. Summers, External Mass-Transfer Rate in Fixed-Bed Adsorption. Journal of Environmental Engineering, 1985. 111(6): p. 891-905.

20. Dwivedi, P.N. and S.N. Upadhyay, Particle-fluid mass transfer in fixed and fluidized beds. Industrial \& Engineering Chemistry Process Design and Development, 1977. 16(2): p. 157165.

21. Karger, J., D.M. Ruthven, and D.I. Theodorou, Diffusion in Nanoporous Materials. 2011, Weinheim: Wiley-VCH.

22. Ruthven, D.M., Principles of Adsorption \& Adsorption Processes. 1984, United States of America: John Wiley \& Sons, Inc.

23. Hand, D.W., J.C. Crittenden, and W.E. Thacker, Simplified models for design of fixed-bed adsorption systems. Journal of Environmental Engineering-Asce, 1984. 110(2): p. 440-456.

24. Ahmad, A.A. and B.H. Hameed, Fixed-bed adsorption of reactive axo dye onto granular activated carbon prepared from waste. Journal of Hazardous Materials, 2010. 175(1-3): p. 298-303.

25. Banat, F., et al., Bench-scale and packed bed sorption of methylene blue using treated olive pomace and charcoal. Bioresource Technology, 2007. 98(16): p. 3017-3025.

26. RohmHaas, Amberlite XAD4, industrial grade polymeric adsorber, product data sheet, 2003.

27. Xu, Z., Q. Zhang, and H.H.P. Fang, Applications of Porous Resin Sorbents in Industrial Wastewater Treatment and Resource Recovery. Critical Reviews in Environmental Science and Technology, 2003. 33(4): p. 363-389.

28. Yan, J., X. Wang, and J. Chen, Swelling of porous styrene-divinylbenzene copolymers in water. Journal of Applied Polymer Science, 2000. 75(4): p. 536-544.

29. Bui, A.V. and M.H. Nguyen, Prediction of viscosity of glucose and calcium chloride solutions Journal of Food Engineering, 2004. 62(4): p. 345-349.

30. Nanoti, A., et al., Process studies for development of adsorption technology for furfural recovery from waste water. Environmental Progress, 2008. 27(3): p. 308-312. 


\section{Very fast adsorption of biological anions by particle loaded mixed matrix membranes}

\subsection{Abstract}

MMMs are seen as a solution to use very small adsorption resins while avoiding high pressure drop and channeling due to difficult packing associated with smaller particles. For small solutes, small resins show comparable adsorption capacities and diffusion constants for large resins, but due to the short path length, overall kinetics are much faster for small resins. This study examines if and how the adsorption capacity and kinetics change when small particles are incorporated into MMMs. A mixed matrix membrane (MMM) loaded with small ion exchange particles $(8 \mu \mathrm{m})$ was prepared. The capacity and kinetic properties of this MMM were compared with those of single ion exchange particles $(8 \mu \mathrm{m}$ and $625 \mu \mathrm{m})$ for differently sized anions. The MMMs showed very good adsorption capacities and incorporation of the small resins into the MMM hardly caused any loss of capacity. The overall adsorption rate of the MMM was increased enormously compared to the adsorption rate of the larger resins $(625 \mu \mathrm{m})$ due to shortening of the diffusion path. Incorporation of small particles in MMMs makes very fast adsorption at very high flow rates possible and MMMs could therefore play a valuable role in downstream processing.

Keywords: Zero length column (ZLC), diffusion, mixed matrix membrane, ion exchange, folic acid, peptide, BSA

\subsection{Introduction}

Chromatographic purification plays an important role in the downstream processing in the pharmaceutical and biotechnology industries [1, 2]. Conventional stationary phases used for these separations have large capacities but are generally not suitable for high linear velocities and therefore large installations are often necessary [3]. Furthermore the resolution is often low. This is 
solved by reducing the size of the adsorbent, but unfortunately this increases the pressure drop over the bed resulting in further reduction of the linear velocity. As a solution, small resins are usually packed in shallow adsorption columns with large diameters (pancake like columns). These are however difficult to pack and still show high pressure gradients over the column. Recently membrane chromatography with particle loaded membranes (mixed matrix membranes, MMMs) is investigated as a solution to use small sized adsorbents with high linear velocities while the pressure drop remains low $[4,5]$

In conventional columns with large particles the transport of the solutes to the particles is by convective transport, while a considerable part of the adsorption time is determined by the slow intra particle diffusion in the pores of the particles (Figure 1A) [1]. When particle loaded MMMs are used in chromatography, transport of the solute mainly takes place by fast convection in the pores of the polymeric membrane structure while slow intra particle diffusion only takes place in the short dead-end pores of the small particles (Figure 1B), resulting in much faster overall adsorption times [6]. Ideally, the pores of the membrane are very well defined and channeling, often observed in conventional columns due to packing of the adsorbents, is prevented. Contrary to conventional columns, the capacity of membranes is achieved by parallel configuration of the membranes rather than stacking of the membranes. Therefore the bed length of membranes is shorter compared to that of columns and the pressure drop over the bed is much smaller. 

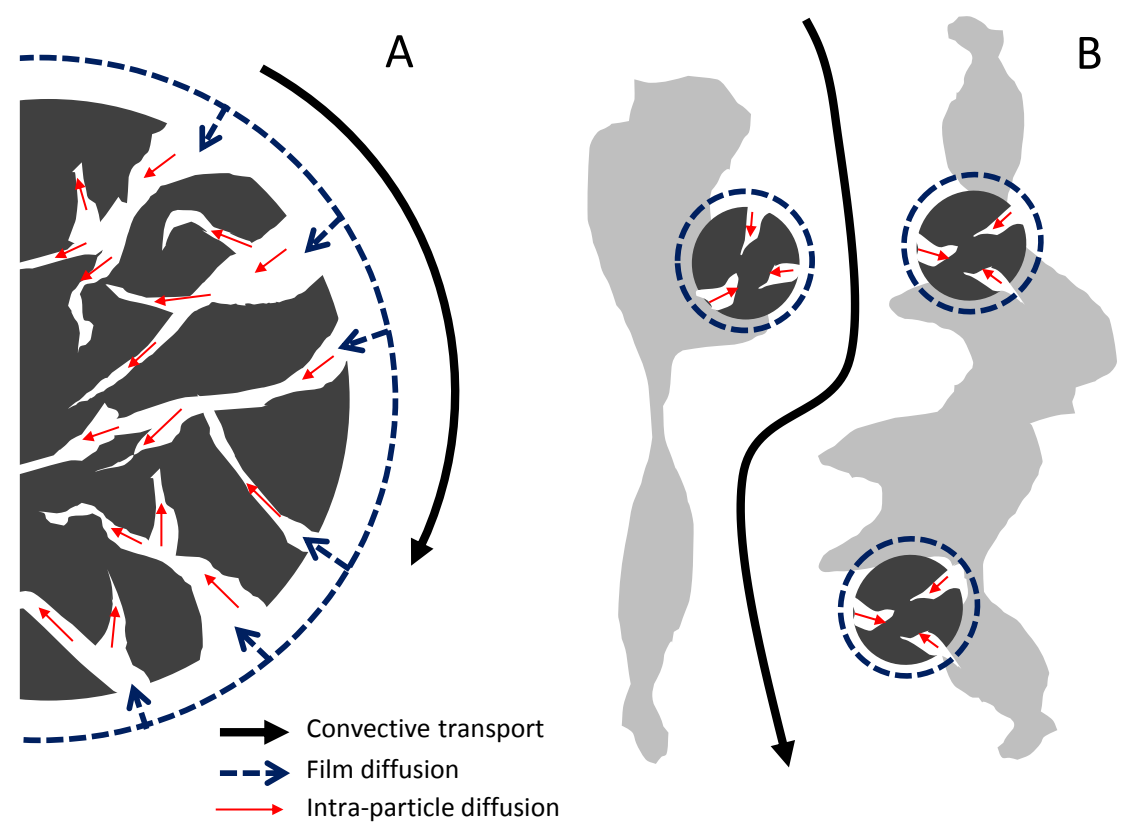

Figure 1: Solute transport in a conventional packed bed with large particles (A) and a pore of a MMM loaded with small particles (B).

For small solutes the faster kinetics of MMM are the main reason to choose MMMs with smaller particles over conventional columns. For larger solutes (e.g. large proteins), which hardly or do not diffuse at all into the adsorbents, the increased capacity due to the extra available surface area because of the smaller particles, is the main reason to choose MMMs. For these solutes diffusion in the pores of the adsorbent is absent and fast adsorption is expected for large and small adsorption particles as well as for MMMs. Furthermore membranes are easily scaled up, are modular in nature and have a low footprint [7].

Studies on differently sized, but otherwise similar resins, show that the diffusion coefficient for all these resins is constant for one single solute $[8,9]$. However, as expected the overall adsorption time decreases significantly for the smaller sized resins. In this study we incorporate small adsorption particles in MMMs and compare the adsorption capacity and kinetics of MMMs with the 
adsorption capacity and kinetics of large and small adsorption resins to study the effect of the incorporation of the small resins in MMMs on the resin properties.

There are different experimental set-ups that can be used to determine adsorption kinetics of adsorbents. Stirred or shaken vessels are most often used. Unfortunately these type of kinetic experiments are unsuitable for our purposes since for MMMs convective transport through the membrane is absent in these experiments and adsorption times are still very long $[4,10,11]$. A solution for this is offered by an alternative method, the zero length column (ZLC) introduced by Eic and Ruthven in 1988 and frequently used to study the adsorption kinetics of adsorbents [12]. In this experiment, a solute is pumped from a vessel over a very short column containing the adsorbent back to the vessel. The short bed length, the flow rate and the solution volume are chosen in such way that the concentration of the solution is almost equal in the entire set up at a given time, mimicking an ideal mixed finite bath. This method is also very suitable to measure the adsorption kinetics of MMMs when the column is replaced by a filter holder with the MMM under investigation. The fast flow through the membrane ensures maximal convective flow through the pores of the membrane minimizing solute transport time by slow diffusion through the membrane pores. As far as we know only scrapings of the surface of alumina-MFI membranes were used as adsorbents in a column for ZLC experiments $[13,14]$. There is very limited literature available on ZLC combined with membranes and to our knowledge it is the first time that ZLC experiments are applied while membranes themselves function as a column, ensuring convective flow through the pores of the membrane.

For the experiments we use three differently sized molecules that show acidic properties at $\mathrm{pH} 7$, folic acid $(441 \mathrm{~g} / \mathrm{mol})$, a synthesized peptide $(1028 \mathrm{~g} / \mathrm{mol})$ and the protein BSA $(64.000 \mathrm{~g} / \mathrm{mol})$ to study the effect of the size of the molecule on the adsorption capacity as well as on kinetics. The properties of the molecules are given in Table 1. The adsorption capacity and kinetics of the selected resin (large and small) and the MMMs containing the small resin particles was studied with the resin Lewatit MP62 (MP62), a styrene based anion exchange resin. 


\begin{tabular}{lccc}
\hline & Mw & pKa & pl \\
\hline Folic acid (FA) [15] & $441 \mathrm{~g} / \mathrm{mol}$ & $-1.5,0.2,2.35,3.46$, & \\
& & $4.56,8.38$ & \\
Peptide (PEP) [16] & $1028 \mathrm{~g} / \mathrm{mol}$ & 2.8 \\
Bovine serum albumin (BSA) & $64.000 \mathrm{~g} / \mathrm{mol}(64 \mathrm{kDa})$ & & 4.7 \\
& & \\
\hline
\end{tabular}

\subsection{Materials and methods}

\subsubsection{Materials}

Polyethersulfone (PES, Ultrason, E6020P) was purchased at BASF. Sodium phosphate monobasic monohydrate (SPM), sodium phosphate dibasic anhydrous (SPD), acetic acid, sodium acetate, folic acid (FA), bovine serum albumin (BSA) and the resins Dowex Optipore L493 (Optipore) and Lewatit MP62 (MP62) were purchased at Sigma Aldrich (the properties are given in Table 2). Polyethyleneglycol with a molecular weight of $400 \mathrm{~g} / \mathrm{mol}$ (PEG400) and NMP were obtained from Merck and Acros organics respectively. The synthetic peptide (PEP) with a sequence DGEWEDYD was synthesized by Caslo ApS. All chemicals were used as received. The resins were rinsed with MilliQ water until impurities were no longer detected in the water with UV-VIS at 200-400 nm. 
Table 2: Resin properties.

\begin{tabular}{|c|c|c|c|c|c|c|}
\hline Resin & Matrix & Type & $\begin{array}{l}\text { Diameter } \\
\text { [ } \mu \mathrm{m}][17]\end{array}$ & $\begin{array}{c}\text { Functional } \\
\text { group }\end{array}$ & $\begin{array}{c}\text { IEX } \\
\text { [min. eq/l] }\end{array}$ & $\begin{array}{c}\text { Surface } \\
\text { area }\left[\mathrm{m}^{2} / \mathrm{g}\right]\end{array}$ \\
\hline Optipore [18] & $\begin{array}{c}\text { Poly(styrene- } \\
\left.\text { DVB }^{*}\right)\end{array}$ & Adsorbent & 557 & None & - & $>1100$ \\
\hline MP62 [19] & $\begin{array}{c}\text { Poly(styrene- } \\
\text { DVB) }\end{array}$ & $\begin{array}{l}\text { Weak base } \\
\text { anion } \\
\text { exchanger }\end{array}$ & 625 & $\begin{array}{l}\text { Tertiary } \\
\text { amine }\end{array}$ & 1.7 & 39.3 [17] \\
\hline
\end{tabular}

\subsubsection{Small resin preparation and analysis}

MP62 was dried in a vacuum oven at $80^{\circ} \mathrm{C}$ (14 hours). To obtain small MP62 resin particles the resin was grinded with a stainless steel ball in a Fergus pulveriser and sieved with a sieve opening of $32 \mu \mathrm{m}$.

Before measuring the particle size of the grinded MP62, the resin was treated with a Branson Sonifier 450 to break down the agglomerates. The particle size of the grinded resin was measured by dynamic light scattering (DLS) experiments at $25^{\circ} \mathrm{C}$ in a Malvern Zetasizer HS3000. A nominal $5 \mathrm{~mW}$ Helium Neon laser with $633 \mathrm{~nm}$ wavelength was used to measure the particle size distributions under angles of 12 and $90^{\circ}$. The correlograms were analyzed using the CONTIN method. The BET particle size of the large MP62 and Optipore resins have been determined earlier [17].

The specific surface area (BET), pore size and pore volume of the grinded MP62 resin was determined by nitrogen adsorption at $-195.8{ }^{\circ} \mathrm{C}$ with the Micromeritics Tristar 3000 . Before the 
measurement, the resin was heated overnight at $80^{\circ} \mathrm{C}$ under nitrogen flow. The BET surface areas of the large MP62 and Optipore resins have been determined earlier [17].

\subsubsection{MP 62 MMM fabrication}

A solution containing 12 wt\% PES, 12 wt $\% 8$ m MP62, 35 wt\% NMP, 35 wt\% PEG400 and 6 wt $\%$ $\mathrm{H}_{2} \mathrm{O}$ was stirred overnight to break down the clusters of particles. PES is used the matrix forming polymer, NMP as the solvent and PEG 400 and $\mathrm{H}_{2} \mathrm{O}$ are additives to tailor the porosity and pore size of the membranes $[22,23]$. Flat sheet membranes were prepared by casting the solution on a glass plate with a $1 \mathrm{~mm}$ casting knife. The membranes were coagulated in a $50^{\circ} \mathrm{C}$ water bath.

\subsubsection{Membrane characterization}

Scanning electron microscopy (SEM)

The membrane morphology was investigated by SEM imaging with a JEOL JSM LV Scanning Electron Microscope. Samples of the cross section of the membranes were obtained by freezing and fracturing a small piece of the membrane in liquid nitrogen. Surface samples were obtained by cutting a small piece of the membrane. The membrane samples were dried overnight in an $80^{\circ} \mathrm{C}$ vacuum oven and then sputtered with gold using a Balzer Union SD 040 sputtering apparatus under argon atmosphere.

\subsubsection{Adsorption properties of the adsorbents}

Adsorption on Optipore and MP62 resins as function of $\mathrm{pH}$

The adsorption mechanism of FA, PEP and BSA on the resins was investigated by comparing the adsorption of these molecules on the large Optipore (a styrene based resin) and the large MP62 (a styrene based anion exchange resin) at different $\mathrm{pH}$. At $\mathrm{pH} 7$ we expect that adsorption on this resin by the solutes is achieved by anion exchange. This is examined by comparing the adsorption 
of the different solutes at different $\mathrm{pH}$ on MP62 and compare it with a the adsorption on Dowex Optipore L493 (Optipore), a styrene based resin without ion exchange groups. The adsorption was measured at $\mathrm{pH} 4$ to 8 , with the exception of $\mathrm{FA}(\mathrm{pH} 6$ to 8 ) because FA's solubility is too low below $\mathrm{pH} 6$ [15]. The $\mathrm{pH}$ was regulated with a $100 \mathrm{mM}$ acetate buffer (acetic acid/sodium acetate) between $\mathrm{pH} 4$ and 5 and with a $100 \mathrm{mM}$ phosphate buffer (SPM/SPD) between pH 6 and $8.20 \mathrm{ml}$ of a $50 \mathrm{~g} / \mathrm{l}$ solution was added to $0.1 \mathrm{~g}$ resin (FA, BSA) or $0.025 \mathrm{~g}$ (PEP). The solutions were placed in a shaking bath at $20^{\circ} \mathrm{C}$ for 48 hours after which the concentration was analyzed with UVVIS.

Adsorption isotherms MP62 (large grinded resins and MP62 MMM)

Solutions (20 ml) of 10 to $100 \mathrm{mg} / \mathrm{FA}, \mathrm{PEP}, \mathrm{BSA}$ in a $100 \mathrm{mM}$ phosporic acid buffer $(\mathrm{pH} 7)$ were added to the MP62 resins (large resins and grinded resins) and one MP62 MMM $\left(4.9 \mathrm{~cm}^{2}\right)$. The solution with the grinded resins were treated with a Branson Sonifier 450 for 5 minutes to break down agglomerates. The solutions were equilibrated for $48 \mathrm{~h}$ in a thermostatic shaking bath at 20 ${ }^{\circ} \mathrm{C}$. The concentration of the solutes was analysed with UV-VIS. The solution was separated from the grinded resins with a syringe filter (Whatman Spartan 13, $0.45 \mathrm{RC}$ ). Adsorption of solutes on the syringe filter was not observed, as was tested separately.

The obtained isotherms were fitted with Freundlich (equation 1) or Langmuir (equation 2) [24]. The Langmuir equation was used when the graphs showed a plateau value. In all the other cases Freundlich was used.

$q=K \cdot c_{e q}{ }^{1 / n}$ (Eq. 1) 
With $\mathrm{q}\left[\mathrm{mg}_{\text {solute }} / \mathrm{g}_{\text {adsorbent }}\right]$ the adsorption capacity at the equilibrium concentration $\mathrm{c}_{\text {eq }}\left[\mathrm{mg}_{\mathrm{solute}} / \mathrm{l}\right], \mathrm{K}$ $\left[1 / g_{\text {adsorbent }}\right]$ the Freundlich equilibrium constant that indicates the maximum adsorption capacity and $1 / n$ the constant measuring the strength of adsorption.

$$
q=\frac{b \cdot c_{e q} \cdot q_{\max }}{1+b \cdot c_{e q}}
$$

With q $\left[\mathrm{mg}_{\text {solute }} / \mathrm{g}_{\text {adsorbent }}\right]$ the adsorption capacity at the equilibrium concentration $\mathrm{c}_{\text {eq }}\left[\mathrm{mg}_{\text {solute }} / \mathrm{l}\right]$, b $\left[1 / \mathrm{mg}_{\text {solute }}\right]$ the Langmuir equilibrium constant that indicates the energy of adsorption, which increases when the strength of the adsorption bond increases and $\mathrm{q}_{\max }\left[\mathrm{mg}_{\text {solute }} / \mathrm{g}_{\text {adsorbent }}\right]$ the maximum adsorption capacity of the resin.

\subsubsection{Kinetic analysis MP62}

\section{ZLC experiments large MP62 resins}

The effective diffusion coefficient of FA, PEP and BSA on MP62 (large resins) was determined with a zero length column (ZLC) set up as depicted in Figure 2 at $20^{\circ} \mathrm{C}$. The ZLC set up consists of an Akta system in recycle mode. The column (Omnifit column, $6.6 \mathrm{~mm}$ internal diameter) was packed with the resin $(625 \mu \mathrm{m})$ with a bed height of $\sim 2 \mathrm{~mm}$ and equilibrated with water. The column was disconnected and the Akta system was filled with FA, PEP or BSA solution (50 mg/l). The solution was buffered with a a $100 \mathrm{mM}$ phosporic acid buffer $(\mathrm{pH} 7)$. After the column was reconnected, the FA, PEP or BSA solution was pumped through the column in recycle mode. The solute concentration was monitored in time by analyzing samples from the feed container with UV-VIS. Samples were taken at regular intervals. The total volume of the system was $13 \mathrm{ml}$. The flow rate was varied from $30 \mathrm{ml} / \mathrm{min}$ to $40 \mathrm{ml} / \mathrm{min}$ to see whether the adsorption graphs overlap and external mass transfer could be neglected. Due to the very high pressure drop of the column packed with small resins it was not possible to perform this experiment with the grinded resins. 


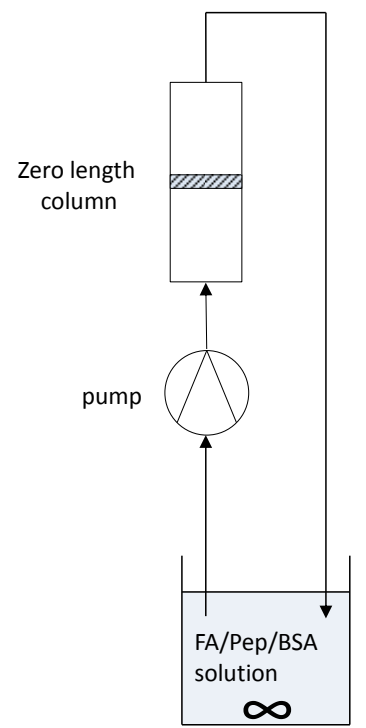

Figure 2: Schematic representation of ZLC set up.

\section{ZLC experiments MP62 MMMs}

The effective diffusion coefficient of the MMMs is as well determined by ZLC experiments and is performed by replacement of the column by a membrane filter holder fitting the the Akta system. One MMM was placed in the $4.9 \mathrm{~cm}^{2}$ filter holder and covered with a paper spacer to ensure an even distribution of the solution over the membrane. The ZLC experimental procedure followed was similar to that of the ZLC experiments for the large resins.

HSDM zero length column experiment

Adsorption of a solute on a macroporous adsorbent consists of several steps. First the solute diffuses from the bulk solution to the boundary layer formed by the stagnant layer surrounding the adsorbent. Then it diffuses through the boundary layer to the surface of the particle, from where it diffuses into the particle. The diffusion in the particle can occur through the pores of the particle (pore diffusion) [25], over the adsorption sites on the pore walls (surface diffusion) [26] or by a combination of both [27]. 
The effective diffusion coefficient cannot be measured directly, but needs to be derived from adsorption data using a kinetic model. In this study the effective diffusion coefficients were modeled with the homogenous solid diffusion model (HSDM) [28]. Diffusion in the HSDM is assumed as diffusion through a homogenous solid sphere. In this case the diffusion coefficient is calculated based on the adsorbent diameter and gives the practical bulk diffusion coefficient, while the actual path length determined by tortuosity is not taken in account. This model is chosen because the specific diffusion mechanism of the solutes into MP62, as well as the tortuosity of the particles is not known.

The HSDM that describes adsorption on spherical particles in a column is based on the mass balance for the adsorbate. The following assumptions are made:

- The diffusion is described by Fick's law.

- There is no concentration gradient over the bed. The bed is very short. This is achieved by using a short bed length ( 2mm) or one mixed matrix membrane.

- The adsorbent is spherical with uniformly distributed pores.

- External mass transfer can be neglected, the flow rate is sufficiently high.

The differential mass balance of a solute in a spherical particle is given by (Equation 3):

$\frac{\partial q}{\partial t}=D_{\text {eff }}\left(\frac{\partial^{2} q}{\partial r^{2}}+\frac{2}{r} \frac{\partial q}{\partial r}\right)$

Were $q$ is the HMF adsorption capacity of the resin $(\mathrm{g} / \mathrm{g}), \mathrm{t}$ is the time $(\mathrm{s}), D_{\text {eff }}$ is the effective surface diffusion coefficient and $r$ the radial position within the particle.

The boundary condition is: 
$\frac{\partial q(r=0, t)}{\partial r}=0$

(Eq. 4)

Signifying that there is no flux across the center of the particle.

With the initial conditions:

$c_{b}(t=0)=c_{0}$

(Eq. 5)

$q(r, t=0)=0$

(Eq. 6)

Were $c_{0}$ is the bulk solute concentration $\left(g_{\text {solute }} /\right)$. The initial conditions of Equation 5 and 6 state that initially the bulk concentration is equal to the initial concentration and that the particle is free of adsorbent.

With these equations the solution for the adsorption curve is obtained and $D_{\text {eff }}$ can be calculated [29] (equation 7).

$\frac{q_{t}}{q_{e q}}=1-\frac{6}{\pi^{2}} \sum_{n=1}^{n} \frac{1}{n^{2}} \exp \left\{-\frac{4 \cdot D_{e f f} \cdot n^{2} \cdot \pi^{2}}{d^{2}} \cdot t\right\}$

With $\mathrm{q}_{\mathrm{t}}$ the solute loading on the resin $\left[\mathrm{g}_{\text {solute }} / \mathrm{g}_{\mathrm{resin}}\right]$ at time $\mathrm{t}[\mathrm{s}]$ and $\mathrm{q}_{\mathrm{eq}}$ the solute loading at equilibrium, $n$ the series of integers, $D_{\text {eff }}\left[\mathrm{m}^{2} / \mathrm{s}\right]$ the effective diffusion coefficient and $d[\mathrm{~m}]$ the diameter of the resin. With the sum of least squares approach, the ZLC adsorption data are fitted with equation 7 to extract $D_{\text {eff }}$.

Finally, the time constant for intraparticle diffusion $\tau_{\mathrm{i}}[\mathrm{s}]$ is calculated as [29]: 


$$
\tau_{i}=\frac{(d / 2)^{2}}{D_{\text {eff }}}
$$

A shorter $\tau_{\mathrm{i}}$ indicates a faster overall adsorption process.

\subsection{Results and Discussion}

\subsubsection{Adsorbent analysis}

Resin properties

The grinded MP62 resins were analysed by DLS and nitrogen adsorption to determine the average particle size and the BET surface area. The results are given in Table 3.

Table 3: Properties Lewatit MP62.

\begin{tabular}{|c|c|c|c|}
\hline & MP62 & MP62 & Optipore \\
\hline & Large resins [17] & Grinded resins & Large resins \\
\hline \multicolumn{4}{|l|}{ Particle size diameter } \\
\hline & 625 & 8 & 557 \\
\hline \multicolumn{4}{|l|}{$[\mu \mathrm{m}]$} \\
\hline \multicolumn{4}{|l|}{ BET surface area } \\
\hline & $39.3 \pm 0.2$ & $37.1 \pm 0.5$ & $>1100$ \\
\hline$\left[\mathrm{m}^{2} / \mathrm{g}_{\mathrm{resin}}\right]$ & & & \\
\hline
\end{tabular}

The average particle size of the grinded resins is $8 \mu \mathrm{m}$. After grinding, the particles were dried and sieved with a sieve size of $32 \mu \mathrm{m}$. With an average size of $8 \mu \mathrm{m}$, the grinded resins are much smaller than the $32 \mu \mathrm{m}$ sieve size and we hypothesize that this is caused by agglomeration of the 
dry grinded resins during sieving. The BET surface area is reduced only little upon grinding. This is likely due to blocking of some of the pores by mechanical deformation of the resin due to the grinding process, however this effect is very limited.

\section{MMM properties}

The SEM micrographs of the MMMs are shown in Figure 3. The micrographs show a very porous structure with well interconnected pores. Evident macrovoids are not observed. The ion exchange particles are held tightly together in the porous matrix, they appear uncoated by the PES and are readily accessible. At both sides of the membrane, pores larger than $0.1 \mu \mathrm{m}$ can be observed. In terms of structure, this membrane is comparable to other successful particle loaded MMMs (e.g. [4, 10, 30]). As those membranes showed excellent adsorption performance, we expected the same for the membranes prepared in this study. 


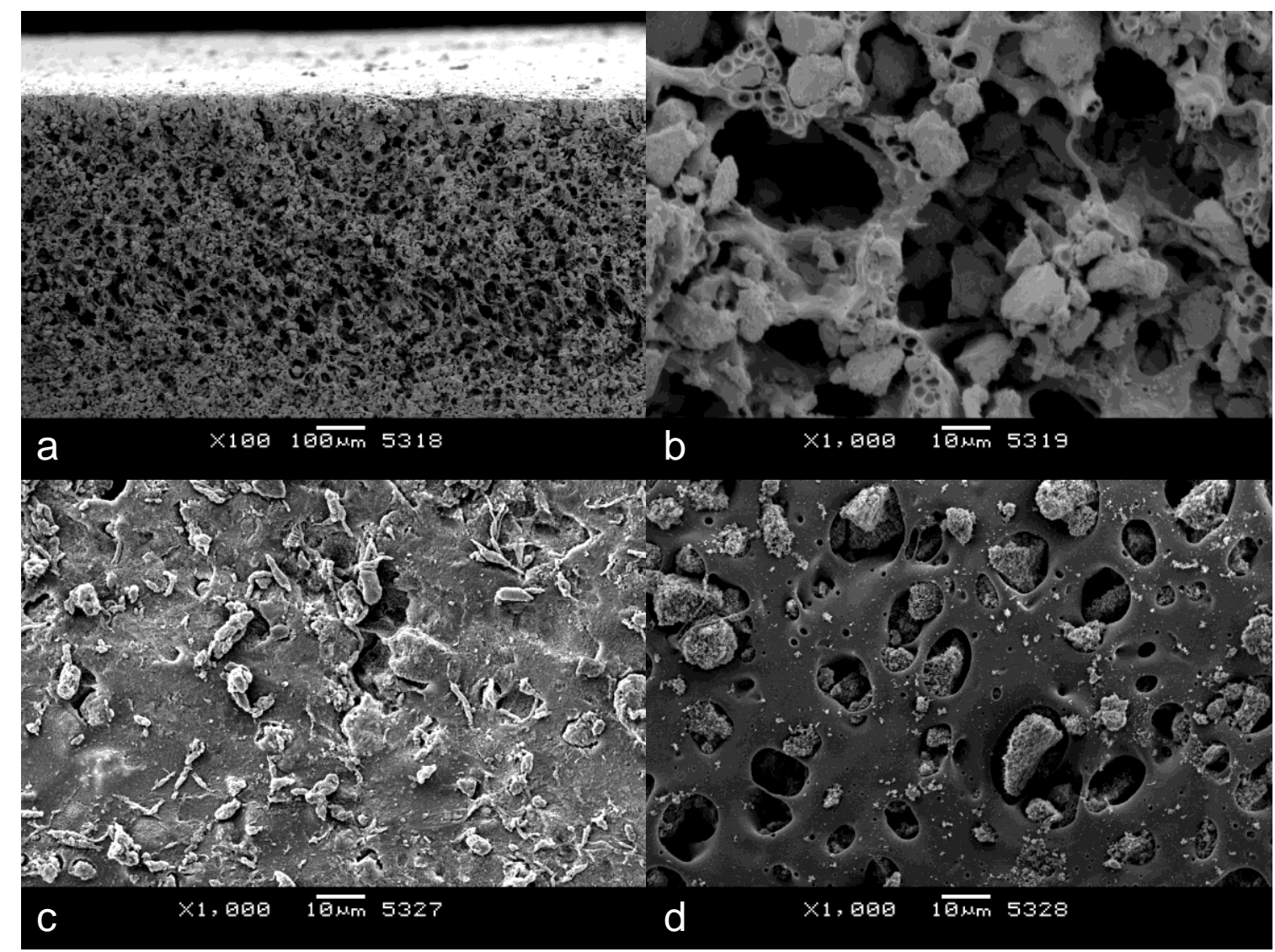

Figure 3: SEM micrographs of membranes prepared from 12 wt\% PES, 12 wt\% MP62, 35 wt\% NMP, 35 wt\% PEG 400 and $6 \mathrm{wt} \% \mathrm{H}_{2} \mathrm{O}$, coagulated in a water bath at $50{ }^{\circ} \mathrm{C}$ : a: cross-section (magnification 100x); b: cross-section (magnification 1000x); c: top-surface (magnification 1000x); d: bottom-surface (magnification 1000x).

\subsubsection{Adsorption properties of the adsorbents}

Adsorption on Optipore and MP62 resins as function of $\mathrm{pH}$

The adsorption mechanism of the selected solute on the resins was investigated by measuring the adsorption of FA, PEP and BSA on Optipore and MP62 at pH 4 to 8 (Figure 4, 5 and 6). 


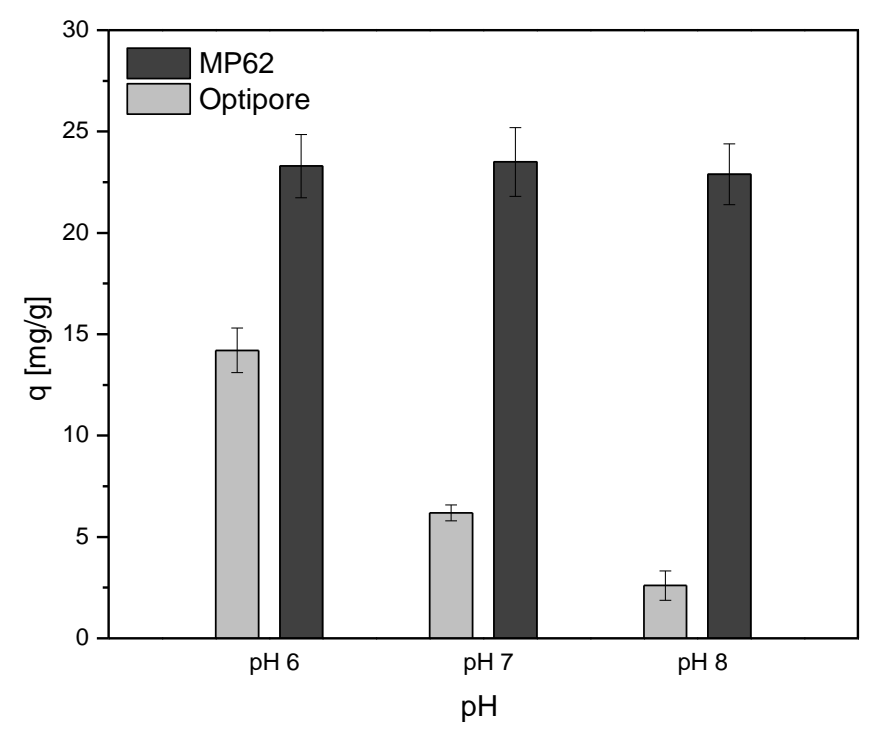

Figure 4: pH effect on FA adsorption capacity of Optipore and MP62.

FA adsorption on Optipore resins is $14 \mathrm{mg}_{\mathrm{FA}} / \mathrm{g}_{\mathrm{resin}}$ at $\mathrm{pH} 6$ and decreases to $3 \mathrm{mg}_{\mathrm{FA}} / \mathrm{g}_{\mathrm{resin}}$ when the $\mathrm{pH}$ is increased to 8. Adsorption on MP62 is higher than on the Optipore resin and remains constant over the $\mathrm{pH}$ range investigated at $\sim 24 \mathrm{mg} \mathrm{FA}_{\mathrm{F}} / \mathrm{g}_{\mathrm{resin}}$.

Higher FA adsorption on hydrophobic Optipore at low $\mathrm{pH}$ than at higher $\mathrm{pH}$ is explained by the increased hydrophobicity of FA due to the varying conformations of the molecule at different $\mathrm{pH}$. At low $\mathrm{pH}$ the hydrophobicity of FA is high, which is reflected in a very low solubility in water at lower $\mathrm{pH}$ [15]. When the $\mathrm{pH}$ increases, the hydrophobicity also decreases shown as an enormous increase of FA solubility in water. The more hydrophobic FA molecule adsorbs well on the hydrophobic styrene matrix of Optipore. As the $\mathrm{pH}$ increases and the hydrophobicity decreases, represented by a greatly enhanced solubility in water, adsorption on Optipore is reduced.

The adsorption of FA on MP62 is much higher compared to the adsorption on Optipore. This is caused by the ionic interactions between FA and the tertiary amine of MP62. And although the surface area of Optipore is much higher $\left(>1100 \mathrm{~m}^{2} / \mathrm{g}\right)$ compared to the surface area of MP62 (39 $\mathrm{m}^{2} / \mathrm{g}$ ) (see Table 2), Optipore shows a much smaller adsorption capacity. This confirms that the ion exchange groups of the resin are mainly responsible for the adsorption of FA on MP62. The 
adsorption on MP62 is independent of the $\mathrm{pH}$ between $\mathrm{pH} 6$ to 8 and is therefore independent on the hydrophobicity of the molecule. This indicates that ion exchange is the main adsorption mechanism. The pKa's of FA show that FA has a constant charge of -1 between $\mathrm{pH} 6$ to 8 . Not much variation in adsorption capacity of the ion exchange resin is therefore expected.

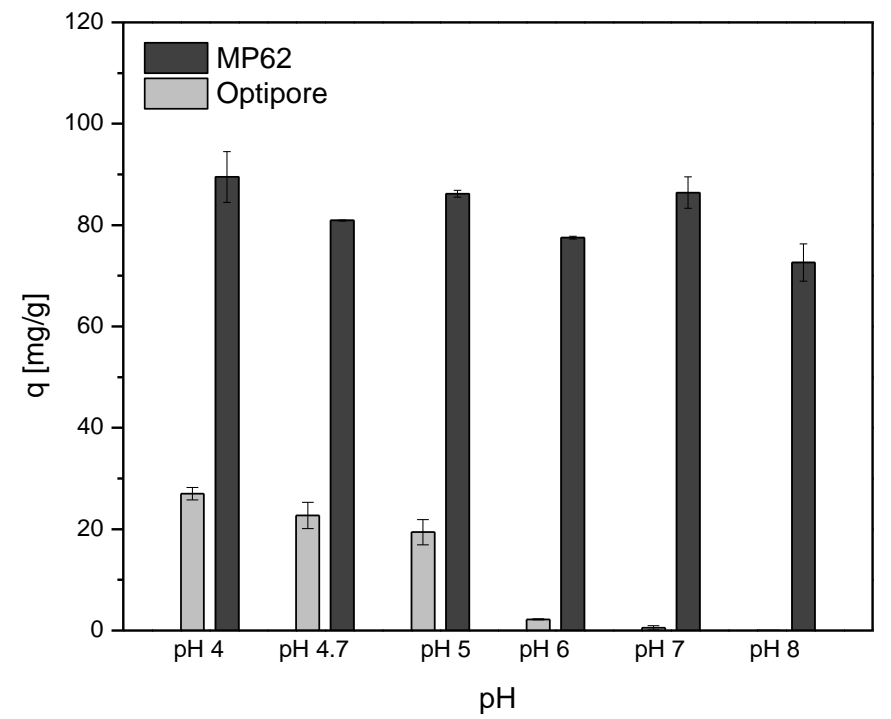

Figure 5: $\mathrm{pH}$ effect on PEP adsorption capacity of Optipore and MP62.

Figure 5 shows that adsorption of PEP in the Optipore resin is $27 \mathrm{mg}$ PEP $/ \mathrm{g}_{\text {resin }}$ at $\mathrm{pH} 4$ and decreases little when the $\mathrm{pH}$ is increased to $\mathrm{pH} 5$. When the $\mathrm{pH}$ is increased to 6 adsorption is minimal and reaches $0 \mathrm{mg}$ PEP $/ \mathrm{g}_{\text {resin }}$ when the $\mathrm{pH}$ is increased further. The adsorption in MP62 is 90 $\mathrm{mg} \mathrm{g}_{\mathrm{PEP}} / \mathrm{g}_{\mathrm{resin}}$ at $\mathrm{pH} 4$ and varies little when the $\mathrm{pH}$ is increased to $\mathrm{pH} 8$.

Adsorption of PEP on Optipore between $\mathrm{pH} 4$ and 5 is achieved by hydrophobic interaction due to the folding of the molecule at the $\mathrm{pH}$ around its isoelectric point [31]. The net charge at the isoelectric point is 0 and the charged groups are folded inside the molecule, compensating each other's charge. The hydrophobic groups are thus positioned at the outside of the molecule. This effect is visible by the minimal solubility that proteins and peptides show around the isoelectric point indicating the increased hydrophobicity. Interaction with the apolar Optipore is then favorable. When the $\mathrm{pH}$ is increased, the number of negative charges in the molecules increases and the 
repulsion of similarly charged groups causes the defolding of the molecule. The polar, charged groups orient themselves towards the outside of the molecule while the apolar, hydrophobic groups are positioned more towards the inside of the molecule. The now accessible polar charged groups of the peptide make interaction with water and interaction with the charged polar groups of the resin more favorable. This results in a higher solubility in water and higher adsorption capacity on MP62, while adsorption on the hydrophobic Optipore is not favorable anymore. Experiments on the solubility of this peptide (not shown) showed that the solubility is indeed reduced when the $\mathrm{pH}$ is reduced from $\mathrm{pH} 7$ to $\mathrm{pH} 4$, supporting the theory of the increased hydrophobicity of the peptide at lower $\mathrm{pH}$.

The relatively low variation in adsorption as function of $\mathrm{pH}$ on MP 62 is surprising. The theoretical isoelectric point of the peptide is 2.8 and the charge is -5 at pH 7 [16]. The charge is therefore not constant over this $\mathrm{pH}$ range, but this apparently has no obvious effect on the adsorption capacity of the peptide on MP62. Several authors have published similar results showing that the ion exchange properties of peptides and proteins cannot be explained by the net charge of the molecule alone $[10,32,33]$. Besides the net charge, the variation in $\mathrm{pH}$ can also alter the distribution and conformation of the charged groups and both also influence the adsorption properties of the molecule [34].

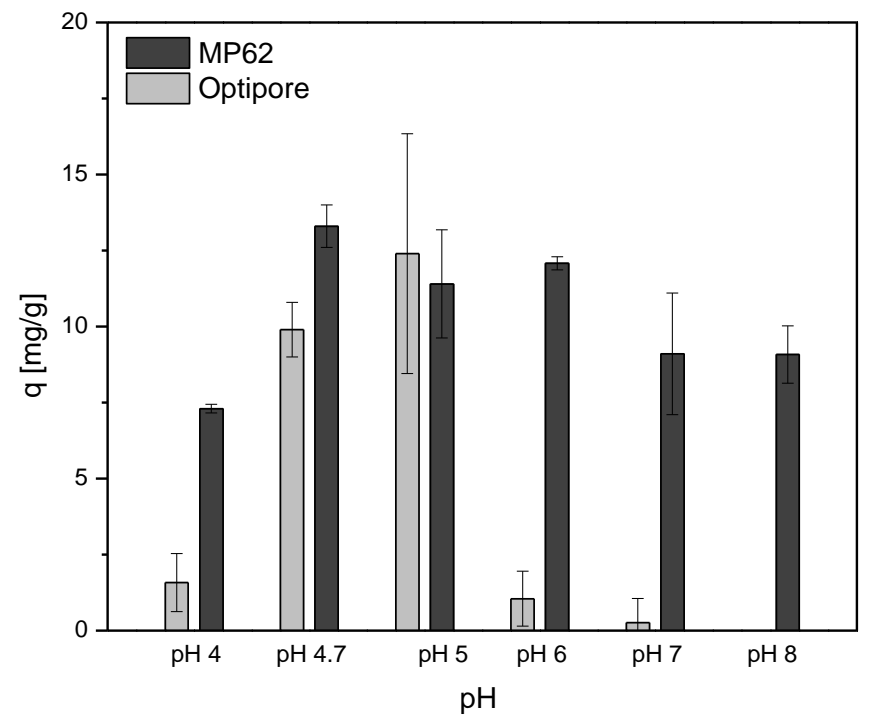

Figure 6: pH effect on BSA adsorption capacity of Optipore and MP62. 
Figure 6 shows that adsorption of BSA on Optipore is low at low pH and increases rapidly around the isoelectric point $(\mathrm{pH} 4.7)$. When the $\mathrm{pH}$ is further increased the adsorption rapidly reduces again to almost $0 \mathrm{mg}_{\mathrm{BSA}} / \mathrm{g}_{\mathrm{resin}}$. In analogy with PEP the hydrophobicity of BSA around the isoelectric point causes the high adsorption capacity of BSA on Optipore and the low adsorption capacity at higher $\mathrm{pH}$. Adsorption of BSA on MP62 remains high upon $\mathrm{pH}$ increase beyond the isoelectric point and seems to show a small peak at the isoelectric point. The molecule is negatively charged when the $\mathrm{pH}$ is higher than the isoelectric point and the adsorption is accomplished through the ion exchange groups of the resin. The relatively low variation of BSA adsorption as function of $\mathrm{pH}$ is consistent with other data of BSA adsorption on ion exchange resins [33] as was discussed for PEP adsorption earlier. The adsorption peak at the isoelectric point is also observed more for proteins $[32,35,36]$. At the isoelectric point, the net charge of the protein is zero, and from a probably oversimplified point of view, BSA adsorption based on the absence of net charge is unexpected on MP62. Often the high adsorption of proteins around the isoelectric point is therefore explained by the reduced size of the protein due to the folding of the molecule around the isoelectric point, allowing more molecules to adsorb on the same available resin area. However we think that, if this is the reason, this phenomenon should also have led to a much higher adsorption on Optipore than we observed, since it has an even larger surface area than MP62. We believe that, since the kinetic diameter of BSA is relatively large (6.2 $\mathrm{nm}$ [37]), the surface charge is most probably not equally distributed over the BSA molecule and localized charges do exist, which are responsible for adsorption on MP62. Furthermore the $\mathrm{pH}$ value of the isoelectric point is obtained from literature and not confirmed by measurements (e.g. zeta potential). Since variations of almost $1 \mathrm{pH}$ unit in isoelectric point of BSA are known due to variation in e.g. salt type and concentration [38], it is unclear whether exactly at $\mathrm{pH} 4.7$, the isoelectric point is indeed reached.

The results of adsorption of FA, PEP and BSA at different $\mathrm{pH}$ shows that adsorption of these solutes at $\mathrm{pH} 7$ on MP62 is predominantly due to the ion exchange groups and not because of the styrene matrix of this resin, since adsorption on the polar matrix of Optipore is absent or minimal at 
this $\mathrm{pH}$. Further experiments exploring the capacity and the kinetics of the resins at $\mathrm{pH} 7$ (large and grinded) and MMMs will therefore only be performed with the MP62 resin and MMM.

\section{Adsorption isotherms}

The adsorption capacity of MP62 adsorbents $(625 \mu \mathrm{m}$ resins, $8 \mu \mathrm{m}$ resins and MMM with $8 \mu \mathrm{m}$ resins) for FA, PEP and BSA (1-50 mg/l) was determined from adsorption isotherms determined at pH 7. The results are given in Figure 7-9. The adsorption isotherms of FA and PEP were modeled with Freundlich and the adsorption isotherms of BSA with Langmuir the results are summarized in Table 4.

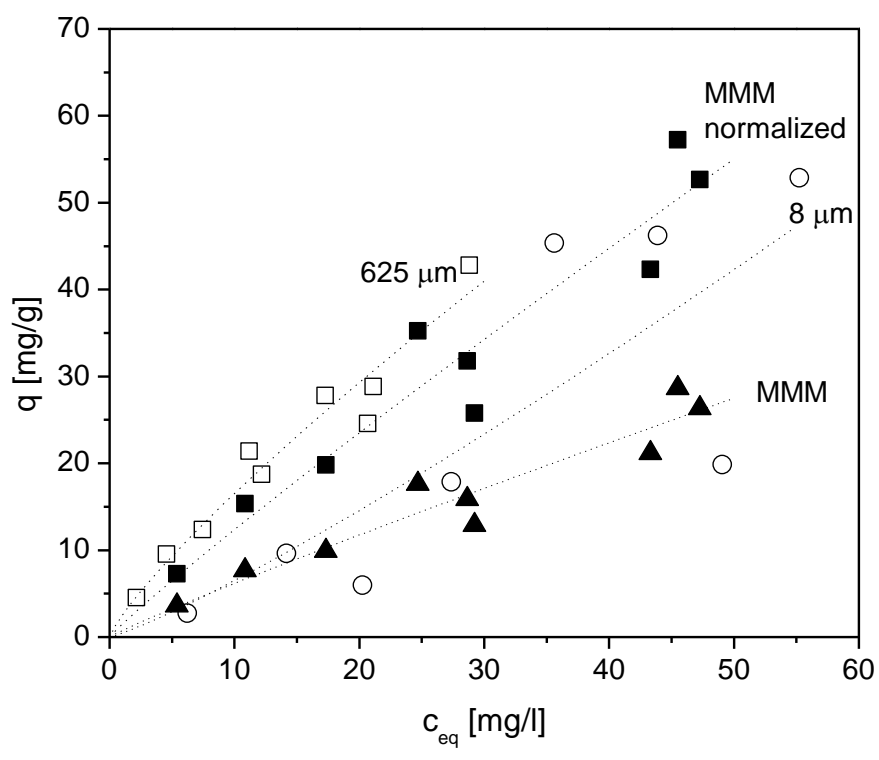

Figure 7: FA adsorption isotherms on MMMs and MP62 resins, $\mathrm{pH} 7\left(\left[\mathrm{C}_{0}\right]=10-100 \mathrm{mg} / \mathrm{l}\right) ; \boldsymbol{\Delta}:$ MMM with $8 \mu \mathrm{m}$ resins, MMM with $8 \mu \mathrm{m}$ resins normalized for resin content, $\square 625 \mu \mathrm{m}$ resins, $\bigcirc: 8 \mu \mathrm{m}$ resins. The dotted lines represent the fit according to the Freundlich isotherms; The obtained Freundlich parameters are summarized in Table 4.

Figure 7 shows that the adsorption isotherms of FA on $8 \mu \mathrm{m}$ and $625 \mu \mathrm{m}$ resins as well as on the MMM are linear and do not show saturation as a plateau value is not reached yet. The data are therefore fitted according to Freundlich (Table 4). The $625 \mu \mathrm{m}$ resin shows the highest adsorption capacity. The trendline of the $8 \mu \mathrm{m}$ resin adsorption isotherm shows that the $8 \mu \mathrm{m}$ resins have a 
slightly lower adsorption capacity than the $625 \mu \mathrm{m}$ resins. It is possible that this is caused by the slightly reduced BET surface area of the $8 \mu \mathrm{m}$ resins (Table 3 ) but this could also be an effect of the large scatter observed in the adsorption of the $8 \mu \mathrm{m}$ resins. FA adsorption on the native PES membrane without resin is not observed. The adsorption on the MMM is therefore accredited to the resins in the membrane. We assume that none of the resins were removed from the MMM during fabrication or washing of the MMMs. The MMM consists of 50 wt $\%$ resins and the adsorption capacity is normalized for this weight percentage. The trendlines show that the normalized adsorption capacity of FA on the MMM is slightly higher than that on the pure $8 \mu \mathrm{m}$ resins, but lower than that on the $625 \mu \mathrm{m}$ resins. Although there are these small differences in the adsorption capacity between the $8 \mu \mathrm{m}$ resins, the $625 \mu \mathrm{m}$ resins and MMM, the adsorption capacities are very comparable. Grinding of the resins affects the adsorption capacity only little and incorporation of the resins in the MMM seems to slightly improve the adsorption capacity possibly due to better accessibility of the small resins.

Activated carbon is known longest for the adsorption of folic acid [39, 40]. It shows similar adsorption capacities as MP62 at lower equilibrium concentration [41] and its capacity it therefore likely higher than that of MP62. However it is also known that the recovery of folic acid from activated carbon is very difficult [39], and other adsorption systems are therefore preferred. Recently Kamran et al. [42] developed magnetic ionic liquid-modified $\mathrm{Fe}_{3} \mathrm{O}_{4}$ nanoparticles (diameter $\sim 10 \mathrm{~nm}$ ) for folic acid adsorption. Although it showed lower adsorption capacities than activated carbon, it performed very well with respect to desorption. MP62 resins show higher adsorption capacities than the $\mathrm{Fe}_{3} \mathrm{O}_{4}$ particles and we will show later that our kinetics achieved in the MMMs are faster than those of $\mathrm{Fe}_{3} \mathrm{O}_{4}$ particles. Furthermore ion exchange resins have shown good folic acid adsorption performance for folic acid concentration from amongst others dairy products [43], blood plasma, erythrocytes, urine [44] and seaweed [45]. 


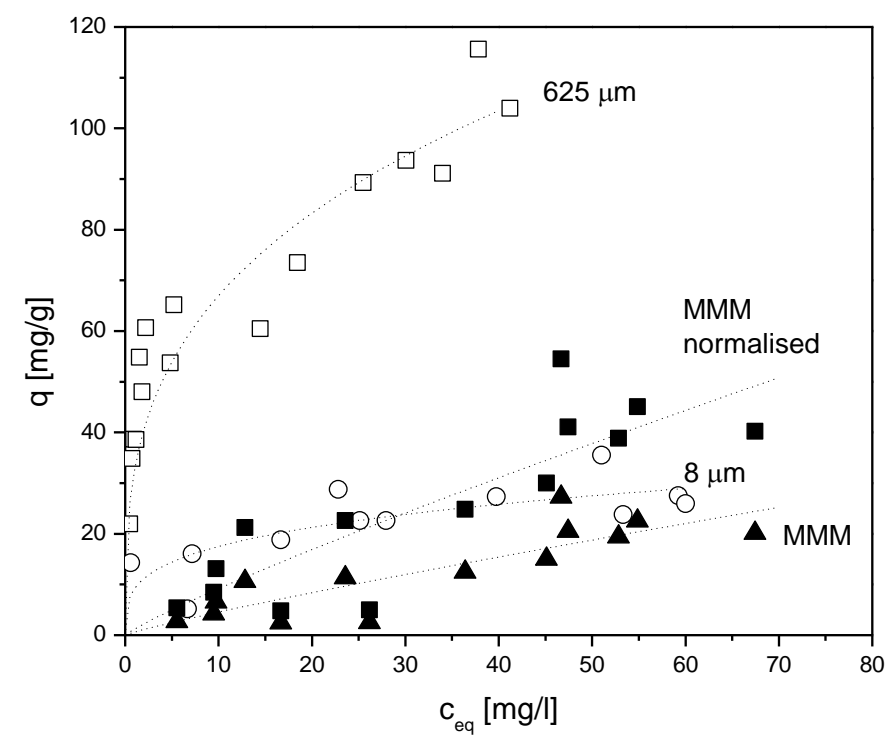

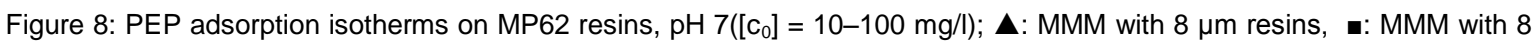
$\mu \mathrm{m}$ resins normalized for resin content, $\square 625 \mu \mathrm{m}$ resins, $\bigcirc: 8 \mu \mathrm{m}$ resins. The dotted lines represent the fit according to the Freundlich isotherms; The obtained Freundlich parameters are summarized in Table 4.

Figure 8 shows the adsorption capacity of PEP on the $8 \mu \mathrm{m}$ resin, the $625 \mu \mathrm{m}$ resin and the MMMs. Again, adsorption on the native PES membrane without resins was not observed. The PEP adsorption was therefore also normalized for the amount of resins in the MMM. The adsorption isotherms were fitted with Freundlich since a plateau value indicating saturation of the adsorbent was not observed. The adsorption capacity of the $625 \mu \mathrm{m}$ resins is much higher than the adsorption capacity of the $8 \mu \mathrm{m}$ resins. Apparently the reduction in adsorption capacity due to grinding of the resins is much higher than what was observed for FA adsorption. Although the reduction in surface area of the $8 \mu \mathrm{m}$ resins is only little (Table 3 ) and the area is still available for small molecules (see FA adsorption), adsorption of PEP, which has a higher Mw than FA, is reduced significantly. This shows that the deformation of the resins by mechanical milling does affect the accessibility of the pores and especially reduces the adsorption capacity available for larger molecules.

Figure 8 also shows that when the particles are incorporated in the MMM, the shape of the curve changes. The normalized adsorption isotherm of the MMM shows a steeper slope than that of the 8 
$\mu \mathrm{m}$ resins. Again it appears that by incorporating the $8 \mu \mathrm{m}$ resin in the MMM, the adsorption sites are a little better accessible to the solute. A possible reason for this could be that in the MMMs agglomeration of smaller particles is prevented as the particles are treated with a sonifier before MMM preparation. Once the particles are embedded in the solid polymer matrix, agglomeration is no longer possible. With the pure $8 \mu \mathrm{m}$ resin, new agglomerates can be formed during the equilibration time of the adsorption experiment. Similar observations were made by Avramescu et al. [4]. The slope of the adsorption isotherm of the PEP curve (Figure 31) is steeper than that of the FA curve (Figure 7) for the $625 \mu \mathrm{m}$ resins indicating that the affinity of the resin for PEP is higher than for FA.

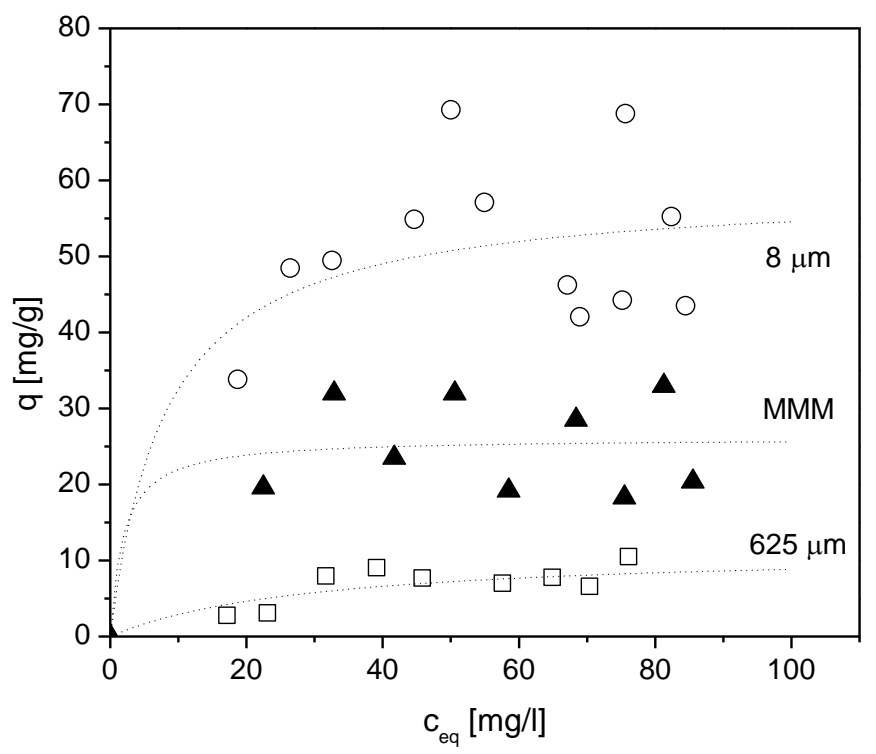

Figure 9: BSA adsorption isotherms on MP62, $\mathrm{pH} 7\left(\left[\mathrm{c}_{0}\right]=10-100 \mathrm{mg} / \mathrm{l}\right) ; \mathbf{\Delta}:$ MMM with $8 \mu \mathrm{m}$ resins, $\square 625 \mu \mathrm{m}$ resins, $\bigcirc: 8$ $\mu \mathrm{m}$ resins, The dotted lines represent the fit according to the Langmuir isotherms; The obtained Langmuir parameters are summarized in Table 4.

Figure 9 shows the adsorption capacity of BSA on the $625 \mu \mathrm{m}$ resin, the $8 \mu \mathrm{m}$ resin and on the MMM. All adsorption isotherms show a Langmuir type adsorption with a plateau value. The $625 \mu \mathrm{m}$ resins have a maximum adsorption capacity of $11.4 \mathrm{mg}_{\mathrm{BSA}} / \mathrm{g}_{\mathrm{resin}}$. The small resins have a maximum adsorption capacity is $59.5 \mathrm{mg}_{\mathrm{BSA}} / \mathrm{g}_{\mathrm{resin}}$. BSA does adsorb on the native PES membrane without 
resins and therefore the adsorption of the MMMs cannot be fully attributed to the resin only. As such the values cannot be normalized for the resin content. As the native PES membrane and the MMM membrane do not have the same porosity and surface area it is not possible to discriminate between the contribution of PES and the resins regarding the adsorption of BSA on the MMM. With $26.1 \mathrm{mg} / \mathrm{l}$, the adsorption capacity of the MMM with the $8 \mu \mathrm{m}$ resins is lower than that of the pure 8 $\mu \mathrm{m}$ resin. The adsorption capacity of the MMM is approximately half the adsorption capacity of the $8 \mu \mathrm{m}$ resin. Since half of the MMM consists of resin, we deduce that the adsorption capacity of the $8 \mu \mathrm{m}$ resin is reduced a bit when incorporated in the MMM. The adsorption capacity of the MP62 MMM is a bit lower than the value of $75 \mathrm{mg} / \mathrm{g}_{\text {MMм }}$ measured by Avramescu et al. [5] on their MP62 MMM. This can be explained by the much higher equilibrium adsorption concentration used in that case.

The capacity of the $8 \mu \mathrm{m}$ resins is increased 5 times compared to the adsorption capacity of the $625 \mu \mathrm{m}$ resins. BSA is a large protein and we assume it only adsorbs on the external surface area of the resin. The surface area is increased by grinding the resins into small particles and the maximum adsorption capacity is therefore increased. Avramescu et al. [4] also measured BSA adsorption on milled particles and MMMs. They used a Lewatit CNP 80 resin in an EVAL matrix and accomplished a 20 times higher adsorption capacity after milling the resins. They expect that this is caused by the increased outer surface area. Nevertheless, it is not clear if some of the BSA can maybe also diffuse into the particles and thus whether the increase is solely due to the enlargement of the outer surface area or also partly due to increased accessibility of the internal pores. The enormous increase in capacity after grinding the resins suggests adsorption on the outer surface is the main mechanism. Some publications show diffusion and adsorption of BSA into macroporous (ion exchange) particles [46, 47] while others show no diffusion and adsorption into the pores of the resins only exhibit adsorption on the outer surface [48]. In general the pore size should at least be 10 times the kinetic diameter of the solute to obtain free transport [49]. The kinetic diameter of BSA is $6.2 \mathrm{~nm}$ [37], the average pore size of MP62 is $40 \mathrm{~nm}$ [17], this would imply that diffusion of BSA into the particle is limited. However, Atyaksheva et al. [37] related the adsorption of BSA on porous silica particles to the pore size and showed that when the pore size is 1.5 to 2 times the diameter of the protein, the protein does diffuse into the pores of the silica 
particles. It should be noted that the authors do not mention the use of a buffer nor the $\mathrm{pH}$ and therefore the exact molecular conformation of the protein is not completely known and might not be comparable to ours. Aggregation of BSA is also mentioned as a phenomenon that hinders pore diffusion. However aggregation is usually observed at low pH [50] and high temperature [51] and not at these low concentrations [52]. Diffusion experiments performed later in this study show that adsorption is very fast and diffusion in the particle is therefore also unlikely. Confocal laser scanning microscopy of cross-sections of the MP62 particles adsorbed with fluorescent labeled BSA could provide the necessary information on the penetration depth of BSA into the particle.

All adsorption isotherms show some scattering. This is due to the relatively low solute concentrations measured in these adsorption experiments and due to low concentration of pollutants released from the adsorbents during the experiments despite intensive washing before use. This influences the solute analysis spectrum by UV-VIS. Although this absolute error is most likely also present in reported adsorption experiments performed with Lewatit resins and MMM (e.g. $[5,10,32])$, the solute concentrations used in our experiments are much lower and therefore the relative error due to pollution is larger resulting in more scatter. Nevertheless, although we do observe scattering in the data, most isotherms show a $R^{2}$ of over 0.95 (Table 4) indicating correlation of the data with Freundlich or Langmuir is sufficiently good to provide good insight in the adsorption capacity of the adsorbent. 
Table 4: Freundlich or Langmuir adsorption isotherm parameters of MP62.

\section{Freundlich}

\begin{tabular}{|c|c|c|c|c|}
\hline Solute & Adsorbent & $\mathrm{K}$ [l//gadsorbent $]$ & $1 / n$ & $\mathbf{R}^{2}$ \\
\hline \multirow[t]{4}{*}{$\overline{F A}$} & $626 \mu \mathrm{m}$ & 2.46 & 0.827 & 0.99 \\
\hline & $8 \mu \mathrm{m}$ & 0.45 & 1.163 & 0.87 \\
\hline & MMM & 0.73 & 0.928 & 0.98 \\
\hline & MMM normalized & 1.45 & 0.928 & 0.98 \\
\hline \multirow[t]{4}{*}{ PEP } & $626 \mu \mathrm{m}$ & 32.51 & 0.314 & 0.98 \\
\hline & $8 \mu \mathrm{m}$ & 9.18 & 0.280 & 0.95 \\
\hline & MMM & 0.60 & 0.880 & 0.91 \\
\hline & MMM normalized & 1.21 & 0.880 & 0.91 \\
\hline
\end{tabular}

Langmuir

\begin{tabular}{|c|c|c|c|c|}
\hline Solute & Adsorbent & $\begin{array}{c}q_{\max } \\
{\left[\mathrm{mg}_{\mathrm{BSA}} / \mathrm{g}_{\text {adsorbent }}\right]}\end{array}$ & b $\left[\mathrm{l} / \mathrm{mg}_{\mathrm{BSA}}\right]$ & $\mathbf{R}^{2}$ \\
\hline \multirow[t]{3}{*}{ BSA } & $626 \mu \mathrm{m}$ & 11.4 & 0.03 & 0.95 \\
\hline & $8 \mu \mathrm{m}$ & 59.0 & 0.12 & 0.97 \\
\hline & MMM & 26.1 & 0.53 & 0.95 \\
\hline
\end{tabular}

Table 4 shows the Langmuir and the Freundlich parameters of the adsorption isotherms. The maximum adsorption capacity determined by Langmuir gives a good insight in the maximum adsorption capacity as determined by the adsorption isotherms. The data fitted with Freundlich 
describe the adsorption isotherms, but have no physical meaning, as the isotherms are mostly linear and consequently $1 / n$ and $K$ are correlated.

\subsubsection{Kinetic analysis of MP62}

ZLC experiments were performed to determine $D_{\text {eff }}$ of the resins and MMMs. The relative adsorption $\left(\mathrm{q}_{t} / \mathrm{q}_{\mathrm{eq}}\right)$ in time is determined (Figure 10-12). For all measurements with FA and PEP, the ZLC adsorption graphs at $30 \mathrm{ml} / \mathrm{min}$ and $40 \mathrm{ml} / \mathrm{min}$ overlap, indicating that the external mass transfer resistance can be neglected and the HSDM model can be applied. In these cases, $D_{\text {eff }}$ is determined by fitting the data using Equation 5 (see Table 5). For BSA on the other hand, pore diffusion is absent and the graphs do not coincide due to very fast adsorption kinetics at the outer surface of the adsorbent. As pore diffusion is absent, the HSDM model is not valid.

Table 5: Effective diffusion coefficients obtained from a fit between the experimental data and the adsorption model.

\section{$625 \mu \mathrm{m}$ resin}

MMM (8 $\mu \mathrm{m})$
$D_{\text {eff }}\left[\mathrm{m}^{2} / \mathrm{s}\right]$
$\tau_{i}[\mathbf{s}]$
$D_{\text {eff }}\left[\mathrm{m}^{2} / \mathrm{s}\right]$
$\tau_{\mathrm{i}}[\mathbf{s}]$

FA

$1.5 \cdot 10^{-11}$

$6.5 \cdot 10^{3}$

$6.7 \cdot 10^{-14}$

$2.4 \cdot 10^{2}$

PEP

$7.5 \cdot 10^{-12}$

$1.3 \cdot 10^{4}$

$1.3 \cdot 10^{-13}$

$1.210^{2}$ 


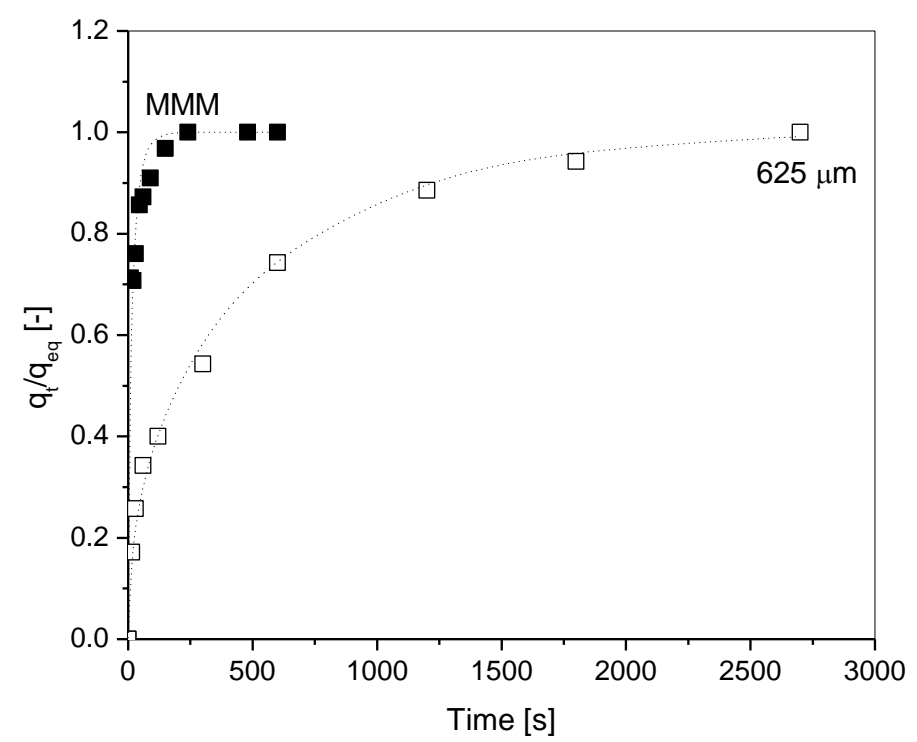

Figure 10: Relative FA loading [q/ $\left./ \mathrm{q}_{\mathrm{eq}}\right]$ in time, $\mathrm{pH}$ 7; $\square: 625 \mu \mathrm{m}$ resins, ..: MMM with $8 \mu \mathrm{m}$ resins. The dotted line represents the fit with HSDM.

Figure 10 shows the relative loading of FA on the resin $(625 \mu \mathrm{m})$ and the MMM. A value of 1 represents the adsorption equilibrium. Figure 33 clearly shows that equilibrium is reached much faster in the MMM than in the $625 \mu \mathrm{m}$ resin. The MMM reaches equilibrium within 2 minutes, while the $625 \mu \mathrm{m}$ resin only reaches equilibrium after 45 minutes. $D_{\text {eff }}$ is $1.5 \cdot 10^{-11} \mathrm{~m}^{2} / \mathrm{s}$ and $6.7 \cdot 10^{-14} \mathrm{~m}^{2} / \mathrm{s}$ for the $625 \mu \mathrm{m}$ resin and the MMM respectively. The time constant for intraparticle diffusion is reduced from $6 \cdot 5 \cdot 10^{3} \mathrm{~s}$ to $2 \cdot 4 \cdot 10^{2} \mathrm{~s}$ in that case, showing the much shorter adsorption time for MMMs.

Unfortunately it was not possible to perform a ZLC experiments with $8 \mu \mathrm{m}$ resins due to the high pressure drop over the bed, even for the short length used here. The size of the resins should not affect the diffusion coefficient $[8,9]$, but only the time constant for intra particle diffusion $\tau_{i}$ is reduced when the size of the resins is reduced. However, since grinding of the resins slightly affects the adsorption capacity, we cannot be guarantee that also the diffusion constant is similar for both the small and the large resins. A change in tortuosity could alter the diffusion coefficient of a resin as determined with HSDM. If we assume that the diffusion constant remains unchanged, the incorporation of the resins in the MMM reduces the diffusion coefficient of FA 200 times when 
the $625 \mu \mathrm{m}$ resins are compared to the MMMs. Since the adsorption capacity of the $8 \mu \mathrm{m}$ resins, the $625 \mu \mathrm{m}$ resins and the resins in the MMM is comparable, we do not believe that this can only be attributed to an altered tortuosity due to the grinding of the resins or blockage of some of the pores by the PES material surrounding the particles. We hypothesize that most likely not all the pores of the MMM show convective transport and that diffusion through the pores of the membrane to the resin does play a role as well in the adsorption process. This increased total path length reduces the adsorption rate in that case. Consequently the calculated diffusion coefficient indicates the time necessary for adsorption, but is not the true diffusion coefficient of the resins in the MMM.

Kamran et al. [42] used magnetic ionic liquid-modified $\mathrm{Fe}_{3} \mathrm{O}_{4}$ nanoparticles (diameter $\sim 10 \mathrm{~nm}$ ) and reached equilibrium in a ZLC experiment in 10 minutes compared to a value of only 2 minutes in our work. This shows that our system is very fast and can compete with other newly developed adsorption particles.

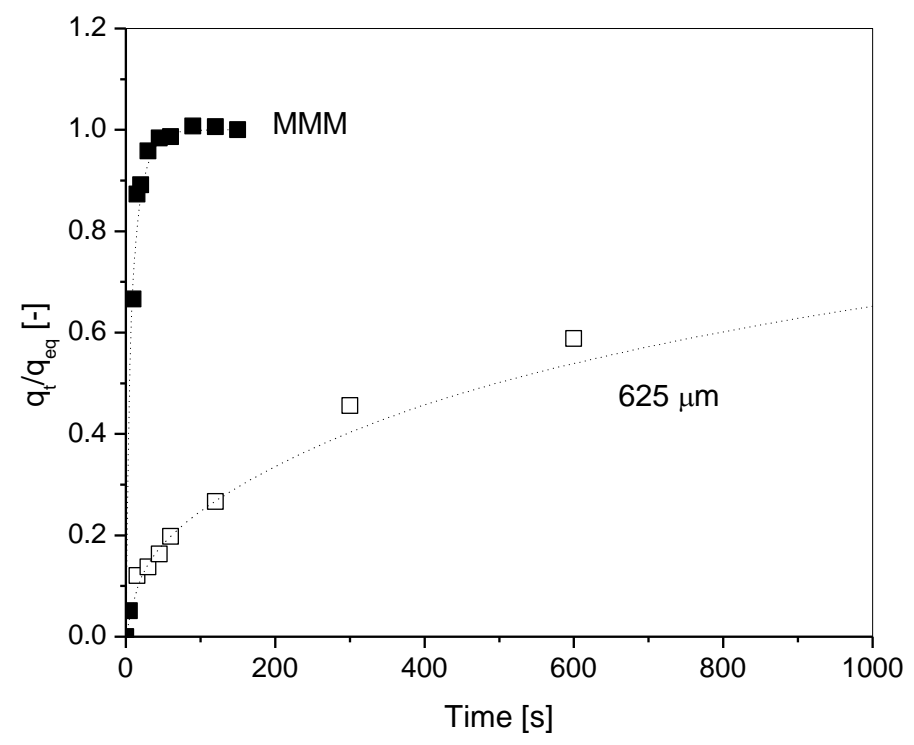

Figure 11: Relative PEP loading [q $\left./ q_{\text {eq }}\right]$ in time; $\square: 625 \mu \mathrm{m}$ resins, . .: MMM. The dotted line represents the HSDM model.

Figure 11 shows the results of the ZLC experiments for the adsorption of PEP. The MMM reaches equilibrium within 1.5 minute, while the $625 \mu \mathrm{m}$ resin requires $\sim 2$ hours to reach equilibrium. The 
calculated effective diffusion constants are $7.5 \cdot 10^{-12} \mathrm{~m}^{2} / \mathrm{s}$ in the $625 \mu \mathrm{m}$ resin and $1.3 \cdot 10^{-13} \mathrm{~m}^{2} / \mathrm{s}$ in the MMM. The time constant for intraparticle diffusion is reduced from $1.310^{4} \mathrm{~s}$ to $1.210^{2} \mathrm{~s}$, showing the much shorter adsorption time for MMMs.

The adsorption of PEP is slower than the adsorption of FA in the large resins, as expected. Based on the molecular weight of the solutes this is expected. The molecular weight of PEP is higher and therefore diffusion in the resin is most likely slower. The modeled $D_{\text {eff }}$ of the $M M M$ is $\sim 60$ times lower than that of the $625 \mu \mathrm{m}$ resins. Assuming that the $625 \mu \mathrm{m}$ and the $8 \mu \mathrm{m}$ have the same macroporous structure and only differ in size, $D_{\text {eff }}$ should be equal in both cases $[8,9]$. Unfortunately $D_{\text {eff }}$ of the $8 \mu \mathrm{m}$ resins could not be determined due to the enormous pressure drop over the column, even in a zero length column experiment.

Unexpectedly the diffusion coefficient of the MMMs is higher for PEP than for FA. We hypothesize that due to the grinding of the resins, some of the smaller pores are blocked for PEP diffusion. Consequently the diffusion path of PEP is shorter than that of FA. This is supported by the reduced adsorption capacity for PEP of the MMM and the $8 \mu \mathrm{m}$ resin, as measured earlier. Although the diffusion coefficient of the MMM is reduced compared to the $625 \mu \mathrm{m}$ resin, the overall kinetics are very good and adsorption is much faster than with the conventionally used large $625 \mu \mathrm{m}$ resin resins. 


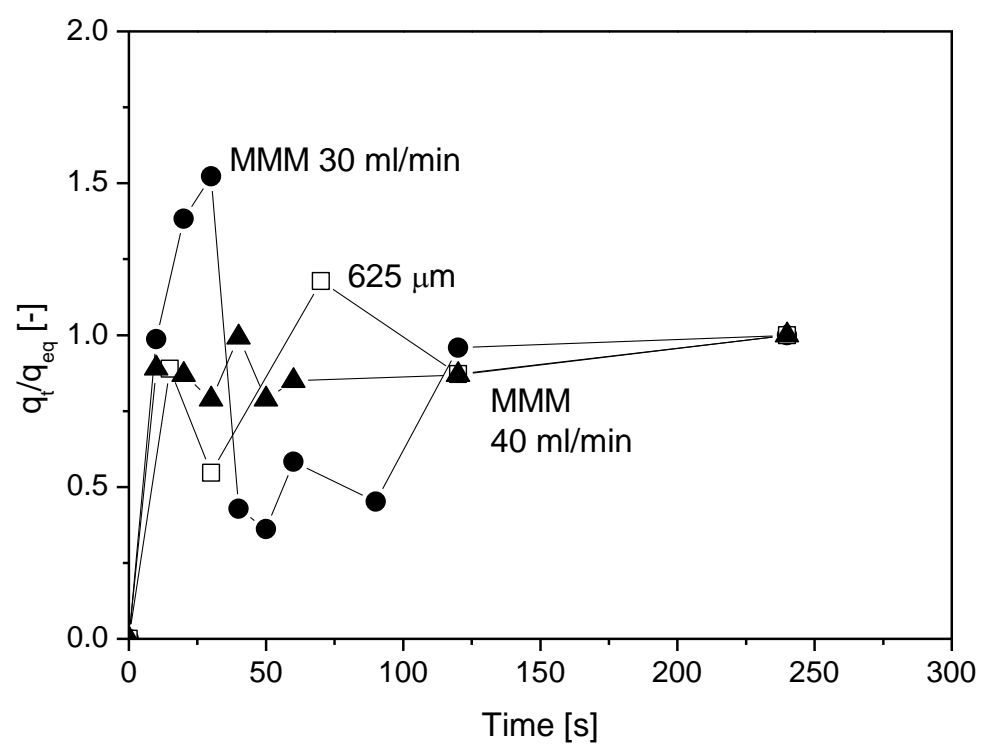

Figure 12: Relative BSA loading [ $\mathrm{q}_{\mathrm{t}} / \mathrm{q}_{\mathrm{eq}}$ ] in time; $\square$ : $625 \mu \mathrm{m}$ resins, $30 \mathrm{ml} / \mathrm{min}, \bullet: \mathrm{MMM}, 30 \mathrm{ml} / \mathrm{min}, \boldsymbol{\Delta} \mathrm{MMM}, 40 \mathrm{ml} / \mathrm{min}$.

The ZLC results of BSA on the large resin and MMM are given in Figure 12. As said, as pore diffusion is absent in this case, the HSDM is not valid. The relative BSA loading on the resin and the MMM shows large fluctuations. This is not only explained by the scatter observed in the adsorption isotherms, but also due to very fast adsorption and desorption of BSA. Initially, the solution pumped to the resins or MMMs has a high concentration BSA resulting in a high driving force for BSA adsorption. Since the feed concentration at that point is higher than the final equilibrium concentration, the adsorption at that point in time is higher than that in the equilibrium state and $q_{t} / q_{\text {eq }}$ reaches a value higher than 1 ( 1 resembles the equilibrium adsorption capacity). The total volume of the system is small. Consequently the concentration of the solution very quickly drops due to the fast adsorption. This low concentration solution is recycled to the resin. Since the feed concentration is then lower than the equilibrium concentration, the driving force for adsorption is opposite and BSA is consequently desorbed again (reducing $\mathrm{q}_{\mathrm{t}} / \mathrm{q}_{\mathrm{eq}}$ ). This process is repeated until equilibrium is reached. This phenomenon is only possible when adsorption is very fast. Since BSA is large and diffusion in the pores is slow, we assume that BSA does not diffuse into the pores of the resin but only adsorbs at the outer surface of the resins. In all experiments performed with the $625 \mu \mathrm{m}$ resins and MMMs, equilibrium is reached within 240 seconds, but most likely 
equilibrium is reached almost instantaneous. Due to the fluctuations in the system, it is not possible to determine the exact adsorption time or kinetics of the resins or MMMs, which can be better examined with a packed bed configuration for this case.

Arevaldo et al. [48] also observed very fast adsorption of BSA on macroporous Lewatit TP 207. They concluded that BSA could not penetrate into the pores of the resin and only adsorbs on the outer surface of the resin. Some resins do show pore diffusion of BSA and in such cases much longer diffusion times are observed in ZLC experiments [46]. Since we believe that pore diffusion does not play a role in our case, the data have not been modelled with the HSDM.

Avramescu et al. [4] measured the diffusion coefficient into an MMM by diffusion only, while convective transport through the pores of the membranes was not applied. It is not clear whether pore diffusion occurred or not. However they expect that BSA mostly adsorbs on the outer surface of the resins because an enormous increase in adsorption capacity after grinding of the resins was observed. For the MMM a diffusion coefficient of $3.410^{-13} \mathrm{~m}^{2} / \mathrm{s}$ was found, but this is the diffusion coefficient of BSA through the pores of the membrane, and not the diffusion coefficient in the pores of the resin, since the thickness of the membrane is chosen as the diffusion length, and only adsorption on the outer surface of the resin is assumed. The kinetic properties of the resins (large or small) were not investigated and a comparison of the diffusion rate in the MMM and the resins is therefore not possible.

SEM analysis shows that our membranes are comparable to obtained by Avramescu et al. in terms of structure and morphology. When we compare the data of Avramescu et al. and our data, it shows that by applying convective transport through the pores of the membrane, the kinetics can be improved enormously and it appears that the long adsorption times in MMMs, observed by Avramescu et al. are significantly improved. The same was observed by Tetala et al. [11] for bovine hemoglobin adsorption on the MMMs with $\mathrm{Cu}^{2+} /$ chitosan particles. However the applied flow rate in that study was very low and adsorption time was not that spectacularly low as observed in our experiments, with equilibrium adsorption capacities reached within 4 minutes compared to a value of 6 hours for Tetala et al. 
The HSDM of the ZLC experiments shows that for FA and PEP the effective diffusion coefficient of the adsorption resins is reduced (200 to 60 times respectively) when the particles are incorporated in a MMM, compared to packing of large resins in a conventional column.

For BSA, which adsorbs at the outer surface of MP62 only, our experiments show no kinetic advantages when MMMs are used. We therefore assume that for large molecules the largest advantage of MMMs, compared to a conventional packed bed, is the increase in adsorption capacity when the smaller particles are used. This combined with the practical application of these small particles in MMMs prevents packing problems and high pressure drops associated with the use of small particles.

Adsorption measurements with MMMs performed earlier [11, 35] for large and small molecules with recirculation of the feed stream already showed that the adsorption times of MMMs decrease when convective transport is applied compared to when diffusion through the MMMs is the only transport mechanism $[4,10,11,35]$. When the adsorption time of MMMs is determined by diffusion only, the adsorption times are very long owing to the long diffusion paths through the pores of the membranes. The performed circulation measurements show that applying a pressure gradient over the membrane reduces the adsorption time. However, due to the low flow rates and large circulation volumes, these experiments did not show the true kinetic potential of MMMs for pressure gradient systems (systems with convective transport through the pores of the membrane). We showed that the application of much higher flow rates reduces the equilibrium adsorption times for MMMs enormously, showing that MMMs are excellent candidates for high throughput adsorption installations.

\subsection{Conclusion}

The adsorption capacity and adsorption kinetics of FA, PEP and BSA at pH 7 on MP62 resins (625 $\mu \mathrm{m}$ and $8 \mu \mathrm{m})$ and MP62 $(8 \mu \mathrm{m})$ loaded MMM's was studied. Comparison of the adsorption capacities of MP62 and that of the apolar Optipore at different pH showed that the adsorption on MP62 at $\mathrm{pH} 7$ is achieved by ion exchange. Defolding of the molecules due to the repulsive negative charges present at $\mathrm{pH} 7$ prevented polar adsorption to the apolar matrix of MP62. 
Small particles were obtained by grinding of the large resins. This grinding process affected the adsorption capacity for the larger molecule PEP, which is probably due to pore blocking of the smaller pores. These pores were still available for FA, since FA adsorption was hardly affected by this. For BSA the adsorption capacity increased by grinding of the resins. This large molecule probably only adsorbs at the outer surface of the resin and as such grinding only enlarges the area available for adsorption.

MMMs loaded with small ion exchange particles showed similar adsorption capacities as the pure small ion exchange particles and the matrix of the membrane does not affect the adsorption capacity. It even appears that by incorporation of the particles in the MMM, agglomeration of the small resins is prevented.

For the adsorbents that diffuse into the resins (FA and PEP), ZLC experiments with large resins and MMMs showed that the overall adsorption rate of the MMMs is improved enormously by incorporation of the small particles in the MMM due to the shorter path length. However, the effective diffusion coefficient of FA and PEP in the MMMs is lower than in the small resins. This indicates an increase in diffusion path length because solutes need to diffuse through some of the pores of the membrane matrix. Because BSA probably only adsorbs on the outer surface of the resins, ZLC experiments are not suitable to determine the adsorption kinetics for BSA on MP62. Nevertheless we did observe that for the large resins as well as for the MMMs the adsorption was very fast.

MMMs show fast overall adsorption kinetics without loss of resin capacity nor high pressure drops and channeling. MMMs therefore allow very fast adsorption at very high flow rates and could therefore play a valuable role in downstream processing.

\subsection{Acknowledgment}

This work was carried out within project SC-00-04 of the Institute for Sustainable Process Technology (ISPT), The Netherlands. 


\subsection{References}

1. Ghosh, R., Protein separation using membrane chromatography: Oportunities and challenges. Journal of Chromatography A, 2002. 952(1-2): p. 13-27.

2. Przybycien, T.M., N.S. Pujar, and L.M. Steele, Alternative bioseparations operations: life beyond packed-bed chromatography. Current Opinion in Biotechnology, 2004. 15(5): p. 469-478.

3. Avramescu, M.-E., Z. Borneman, and M. Wessling, Membrane chromatography, in Handbook of Membrane Separations; Chemical, Pharmaceutical, Food and Biotechnological Applications, A.K. Pabby, S.S.H. Rizvi, and A.M. Sastre, Editors. 2008, CRC Press.

4. Avramescu, M.-E., Z. Borneman, and M. Wessling, Mixed-matrix membrane adsorbers for protein separation. Journal of Chromatography A, 2003. 1006(1-2): p. 171-183.

5. Avramescu, M.-E., Z. Borneman, and M. Wessling, Dynamic behavior of adsorber membranes for protein recovery. Biotechnology and Bioengineering, 2003. 84(5): p. 564572.

6. Orr, V., et al., Recent advances in bioprocessing application of membrane chromatography. Biotechnology Journal, 2013. 31(4): p. 450-465.

7. Drioli, E. and L. Giorno, Comprehensive Membrane Science and Engineering. Vol. 1. 2010, United Kingdom: Elsevier.

8. Djekic, T., Effective intraparticle diffusion coefficients of $\mathrm{CoCl}_{2}$ in mesoporous functionalized silica adsorbents. Adsorption, 2007. 13(3-4): p. 231-237.

9. Schouten, N., et al., Kinetic analysis of anionic surfactant adsorption from aqeous solution onto activated carbon and layered double hydroxide with the zero length column method. Separation and Purification Technology, 2009. 68(2): p. 199-207.

10. Saiful, Z. Borneman, and M. Wessling, Enzyme capturing and concentration with mixed matrix membrane adsorbers. Journal of Membrane Science, 2006. 280(1-2): p. 406-417.

11. Tetala, K.K.R., et al., A metal ion charged ixed matrix membrane for selective adsorption of hemoglobin. Separation and Purification Technology, 2013. 115(0): p. 20-26.

12. Eic, M. and D.M. Ruthven, $A$ new experimental technique for measurement of intracrystalline diffusivity. Zeolites, 1988. 8(1): p. 40-45.

13. Jiang, M. and M. Eic, Transport properties of ethane, butanes and their binary mixtures in MFl-type zeolite and zeolite-membrane samples. Adsorption, 2003. 9(3): p. 225-234.

14. Jiang, M., et al., Diffusion of $n$-butane, isobutane and ethane in a MFl-zeolite membrane investigated by gas permeation and ZLC measurements. Separation and Purification Technology, 2001. 25(1-3): p. 287-295.

15. Wu, Z., et al., Solubility of folic acid in water at $\mathrm{pH}$ values between 0 and 7 at temperatures (298.15, 303.15, and 313.15) K. Journal of Chemical \& Engineering Data, 2010. 55(9): p. 3958-3961.

16. Caslo, Personal communication, 2011.

17. IJzer, A.C., et al., Performance analysis of aromatic adsorptive resins for the effective removal of furan derivatives from glucose. Journal of Chemical Engineering and Biotechnology, 2014. 90: p. 101-109.

18. Dow, Product information Dowex Optipore L493 and V493. Product information Dowex Optipore L493 and V493.

19. Lanxess, Product information Lewatit MP 62. Product information Lewatit MP 62, 2010.

20. Ramaswamy, S., H.-J. Huang, and B. Ramarao, in Separation and Purification Technologies in Biorefineries. 2013, John Wiley \& Sons, Ltd. p. 1-36.

21. Vern, C., et al., The beet sugar factory of the future. International Sugar Journal, 1995. 97(1159): p. 310-316.

22. Smolders, C.A., et al., Microstructures in phase-inversion membranes. Part 1. Formation of macrovoids. Journal of Membrane Science, 1992. 73(2-3): p. 259-275.

23. Torrestiana-Sanches, B., R.I. Ortiz-Basurto, and E. Brito-De La Fuente, Effect of nonsolvents on properties of spinning solutions and polyethersulfone hollow fiber ultrafiltration membranes. Journal of Membrane Science, 1999. 152(1): p. 19-28.

24. Thomas, W.J. and B. Crittenden, Adsorption Technology and Design. 1998: Butterworth Heinemann.

25. Weber, W.J. and E.H. Smith, Simulation and design models for adsorption processes. Environmental Science \& Technology, 1987. 21(11): p. 1040-1050. 
26. Traegner, U. and M. Suidan, Evaluation of surface and film diffusion coefficients for carbon adsorption. Water Research, 1989. 23(3): p. 267-273.

27. Susu, A.A., Mathematical modelling of fixed bed adsorption of aromatics and sulphur compounds in kerosene deodorisation. Chemical Engineering and Processing: Process Intensification, 2000. 39(6): p. 485-497.

28. Qiu, H., et al., Critical review in adsorption kinetic models. Journal of Zhejiang University A, 2009. 10(5): p. 716-724.

29. Karger, J., D.M. Ruthven, and D.I. Theodorou, Diffusion in Nanoporous Materials. 2011, Weinheim: Wiley-VCH.

30. Borneman, Z., Particle loaded membrane chromatography, in Ph.D. thesis2006, University of Twente: Enschede.

31. Berg, J., J. Tymoczko, and L. Stryer, Biochemistry. 5th edition ed. 2002, New York.

32. Saufi, S.M. and C.J. Fee, Fractionation of b-Lactoglobulin from whey by mixed matrix membrane ion exchange chromatography. Biotechnology and Bioengineering, 2009. 103(1): p. 138-147.

33. Staby, A., et al., Comparison of chromatographic ion-exchange resins: VI. Weak anionexchange resins. Journal of Chromatography A, 2007. 1164(1-2): p. 82-94.

34. Hashim, M.A., K.-H. Chu, and P.-S. Tsan, Efects of ionic strength and $\mathrm{pH}$ on the adsorption equilibria of lysozyme on ion exchangers. Journal of Chemical Technology \& Biotechnology, 1995. 62(3): p. 253-260.

35. Saiful, Mixed matrix membrane adsorbers for protein and blood purification, 2007, University of Twente: Enschede.

36. Yamamoto, S. and T. Ishihara, lon-exchange chromatography of proteins near the isoeletric points. Journal of Chromatography A, 1999. 852(1): p. 31-36.

37. Atyaksheva, L.F., et al., Adsorption properties of hemoglobin. Russian Journal of Physical Chemistry A, 2012. 86(3): p. 468-474.

38. Salis, A., et al., Measurements and theoretical intrepetation of points of zero charge/potential of BSA protein. Langmuir, 2011. 27(18): p. 11597-11604.

39. Mitchell, H.K., E.E. Snell, and R.J. Williams, Folic acid I concentration from spinach 1a. Journal of the American Chemical Society, 1944. 66(2): p. 267-268.

40. Frieden, E.H., H.K. Mitchell, and R.J. Williams, Folic acid II studies on adsorption. Journal of the American Chemical Society, 1944. 66(2): p. 269-271.

41. Shen, W., et al., Surface modification of activated carbon fiber and its adsorption for vitamin B1 and folic acid. Colloids and Surfaces A: Physiochemical and Engineering Aspects, 2008. 331(3): p. 263-267.

42. Kamran, S., M. Asadi, and G. Absalan, Adsorption of folic acid, riboflavin, and ascorbic acid from aqeous sampes by $\mathrm{Fe}_{3} \mathrm{O}_{4}$ magnetic nanoparticles using ionic liquid modifier. Analytical methods, 2014. 6(3): p. 798-806.

43. van Wyk, J. and T.J. Britz, A rapid high-performance liquid chromatography (HPLC) method for the extraction and quantification of folates in dairy products and cultures of propionibacterium freudenreichii. African Journal of Biotecnology, 2012. 11(8): p. 20872098.

44. Monch, S., et al., Quantitation of folates and their catabolites in blood plasma, erythocytes, and urine by stable isotope dilution assays. Analytical Biochemistry, 2010. 398(2): p. 150160.

45. Rodrígues-Benaldo de Quirós, A., et al., Determination of folates in seaweeds by highperformance liquid chromatography Journal of Chromatography A, 2004. 1032(1-2): p. 135-139.

46. Hunter, A.K. and G. Carta, Protein adsorption on novel acrylamido-based polymeric ion exchangers: II. Adsorption rates and column behavior. Journal of Chromatography A, 2000. 897(1-2): p. 81-97.

47. Miyabe, K. and G. Guiochon, Kinetic study of the concentration dependence of the mass transfer rate coefficient in anion-exchange chromatography of bovine serum albumin. Biotechnology Progress, 1999. 15(4): p. 740-752.

48. Arévalo, E., et al., Equilibrium and simulation of the operation for the adsorption of albumin proteins in an iminodiacetic-Cu bounded ion exchange resin (IMAC). Separation and Purification Technology, 2000. 18(3): p. 217-225.

49. Jungbauer, A., Chromatographic media for bioseparation. Journal of Chromatography A, 2005. 1065(1): p. 3-12. 
50. Bhattacharya, M., N. Jain, and S. Mukhopdhyay, Insights into the mechanism of aggregation and fibril formation from bovine serum albumin. Journal of Physical Chemistry B, 2011. 115(14): p. 4195-4205.

51. Yohannes, G., et al., Thermal aggregation of bovine serum albumin studied by asymmetrical flow field-flow fractionation. Analytica Chimica Acta, 2010. 675(2): p. 191198.

52. Fuentes, M., et al., Adsorption behavior of bovine serum albumin on lowly activated anionic exchangers suggests a new strategy for solid-phase proteomics. Biomacromolecules, 2006. 7(4): p. 1357-1361. 


\section{Particle loaded mixed matrix membranes for high throughput HMF adsorption applications}

\subsection{Abstract}

HMF is a toxic byproduct produced during the fermentation of biomass for the production of ethanol or other valuable chemicals. HMF can successfully be removed using a packed bed adsorption column containing the resin Dowex Optipore L-493. Conventional packed beds show large capacities but are generally not suitable for high linear velocities and therefore need large installations. Particle loaded mixed matrix membranes (MMMs) are recognized as alternative stationary phases to overcome the problem associated with conventional packed beds.

A MMM loaded with small grinded Optipore resins was prepared. The capacity of the small $(15 \mu \mathrm{m})$ and the original resin $(557 \mu \mathrm{m})$ was compared to that of the MMM containing the same resin (15 $\mu \mathrm{m})$ for HMF adsorption. All particles showed comparable adsorption capacities. Zero length column experiments showed that the MMMs showed very fast overall adsorption kinetics. This was confirmed by breakthrough experiments that showed that MMMs could be operated and even improve the adsorption performance of the resin, using very high flow rates.

\subsection{Introduction}

The production of chemicals from biomass feedstock receives extensive attention recently. A biobased feedstock for renewable fuels and base chemicals is lignocellulosic biomass. The cellulose and hemicellulose of the biomass can be hydrolysed into sugars which can be further treated to produce ethanol and other valuable chemicals [1]. During the production of sugar, toxic byproducts such as acetic acid, phenolic compounds and furans are produced [2]. Previously we showed that the furan derivative 5 hydroxymethylfurfural (HMF) could be successfully removed from sugar (glucose) with an adsorption process using the hypercrosslinked resin Dowex ${ }^{\mathrm{TM}}$ Optipore $^{\mathrm{TM}}$ L-493 (Optipore) [3]. The apolar styrene matrix of the Optipore resin showed excellent 
HMF adsorption capacity, while glucose was not adsorbed. Furthermore the resin showed much better adsorption kinetics than other comparable resins such as XAD4 [4].

Conventional stationary phases such as adsorption columns, show large capacities but are generally not suitable for high linear velocities and therefore need large installations [5]. This is caused by the long diffusion distances in the resin. The transport of the solutes to the particles occurs through convective transport, while a considerable part of the adsorption time is determined by the slow intra particle diffusion in the particles [6]. Although smaller particles could be used, these result in very high pressure drops over the column, making this approach not suitable. A solution for this is the use of very small adsorption particles $(\sim 10 \mathrm{~nm})$ incorporated in so-called mixed matrix membranes (MMMs) [7-9]. The small particles reduce the diffusional path of the solute in the resin (and thereby the adsorption time) significantly, while the flow through the bed and along the particles is improved simultaneously because of the porous structure of the membrane. Zero length column measurements with MMMs for the adsorption of small biological molecules showed that the overall adsorption kinetics of resins, as measured with ZLC experiments, could be improved tremendously using MMMs [9]. When these MMMs are used instead of an conventional column the flow rate and therefore the throughput can be improved.

In this research we prepared MMMs with small Dowex Optipore Resins. Earlier research on MMMs for small adsorbents showed that the overall adsorption rate could be improved by using MMMs [9]. In this research the newly developed MMMs are tested for HMF adsorption to study the effect of incorporating an already excellent performing resin in a MMM. Adsorption isotherms were determined and ZLC experiments were performed on these MMMs to study the adsorption properties of these membranes. The data are complimented with breakthrough experiments to study the high throughput possibilities for HMF separation. The results are compared with earlier obtained data obtained with conventional resins $[3,4]$.

\subsection{Materials and methods}

\subsubsection{Materials}

The resin Dowex Optipore L-493 (Optipore), 5-hydroxymethylfurfural (HMF) and sodiumhypochlorite ( $\mathrm{NaOCl}, 10 \mathrm{wt} \%)$ were purchased from Sigma Aldrich. Polyethersulfone (PES, 
Ultrason, E6020P) was obtained from BASF, polyvinylpyrolidone with different molecular weights $\left(P P_{12}, M w 3500 \mathrm{~g} / \mathrm{mol} ; \mathrm{PVPk}_{30}, M w 40.000 \mathrm{~g} / \mathrm{mol} ; \mathrm{PVPk}_{90}, M w 360.000 \mathrm{~g} / \mathrm{mol}\right.$ ) from Fluka, polyethyleneglycol (PEG400) from Merck and N-methylpyrolydone (NMP) from Acros Organics.

\subsubsection{Small resin fabrication}

The small resins were obtained by grinding the purchased Optipore resins with a stainless steel ball in a Fergus pulveriser and sieved with a sieve opening of $32 \mu \mathrm{m}$. Prior to grinding, the resin was dried in a vacuum oven at $80^{\circ} \mathrm{C}(14$ hours $)$.

\subsubsection{MMM fabrication}

Mixed matrix membranes were prepared with a wet phase inversion procedure adapted from the procedure as described by Borneman [10]. PES (24.5 wt\% of solid material), PVPk ${ }_{12}$ (5 wt\% of solid material), $\mathrm{PVPk}_{30}$ (5 wt $\%$ of solid material), $\mathrm{PVPk}_{90}$ (5 wt $\%$ of solid material) and NMP were mixed and stirred with a mechanical stirrer for 24 hours at $50^{\circ} \mathrm{C}$. The percentage NMP was varied to optimize the MMM morphology by using 100,120 or $150 \mathrm{wt} \%$ NMP relative to the solid material NMP (membrane 1-3 respectively). PES was used as the matrix forming polymer, PVP as an additive that is used to improve the interconnectivity of the pores. The polymer solution was cooled to room temperature and $24.5 \mathrm{wt} \%$ of the solid material of the grinded resin particles and $36 \mathrm{wt} \%$ of solid material PEG400 were added to the solution. The solution was again stirred with a mechanical stirrer for 24 hours to break down the particle clusters. The PES and the Optipore resin were added in a $1: 1 \mathrm{wt} \%$ ratio in order to obtain MMMs containing $50 \mathrm{wt} \%$ resin. PEG400 was added as a non-solvent to tailor the porosity. After stirring the solution was left to degas for 24 hours. The membranes were prepared by casting the polymer solution on a glass plate with a 0.5 $\mathrm{mm}$ casting knife after which they were immersed in a coagulation bath containing MilliQ water (74 $w t \%)$ and NMP (26 wt\%). After precipitation the membranes were rinsed with water and placed in a solution of $4 \mathrm{~g} / \mathrm{l} \mathrm{NaOCl}$ for 24 hours to break down the remaining PVP. Finally the membranes were rinsed with MilliQ water. 


\subsubsection{Adsorbent analysis}

Particle size measurement of grinded Optipore

The grinded Optipore resins were treated with a Branson sonifier to break down agglomerates. The particle size was determined by dynamic light scattering (DLS) at $25{ }^{\circ} \mathrm{C}$ in a Malvern Zetasizer HS3000. A nominal $5 \mathrm{~mW}$ Helium Neon Laser with $633 \mathrm{~nm}$ wavelength was used to measure the particle size distribution under angles of 12 and $90^{\circ}$. The correlograms were analysed using the CONTIN method.

\section{Nitrogen adsorption of grinded Optipore}

The grinded Optipore resins were heated overnight at $80{ }^{\circ} \mathrm{C}$ before the BET surface area was determined with nitrogen adsorption at $-195.8 \mathrm{C}$ with the Micromeritcs Tristar 3000.

\section{Scanning Electron Microscope analysis of the MMMs}

The membrane morphology was investigated with a JEOL JSM 5600 LV Scanning Electron Microscope. Surface samples were acquired by cutting a small piece of membrane. Cross section samples of the membranes were obtained by freezing and fracturing a small piece of membrane in liquid nitrogen. The membrane samples were dried overnight in an $80^{\circ} \mathrm{C}$ vacuum oven and then sputtered with gold using a Balzer Union SD 040 sputtering apparatus under argon atmosphere.

\subsubsection{Adsorption isotherms}

Solutions of 3-30 g/l HMF were added to the grinded Optipore and the MMMs. Adsorption isotherms of the large Optipore resins were obtained from previously performed research [3]. The solutions with the grinded resins were treated with a Branson Sonifier 450 for 5 minutes to break down agglomerates. All solutions were equilibrated for $24 \mathrm{~h}$ in a thermostatic shaking bath at $20^{\circ} \mathrm{C}$. The HMF concentration of the solutes was analysed with UV-VIS at $284 \mathrm{~nm}$. The solution was separated from the grinded resins with a syringe filter (Whatman Spartan 13, $0.45 \mathrm{RC}$ ). Adsorption of HMF on the syringe filter was not observed, as was tested separately. 


\subsubsection{Kinetic analysis}

Zero length column experiments MMMs

The effective diffusion coefficient of HMF on MMMs was determined with a zero length column (ZLC) set up with a membrane filter holder (diameter $25 \mathrm{~mm}$ ) as described in earlier work [9]. The HMF concentration was $5 \mathrm{mg} / \mathrm{l}$ and the concentration was monitored in time by analyzing samples from the feed container with UV-VIS at $284 \mathrm{~nm}$. The total volume of the system was $13 \mathrm{ml}$. The data were compared with data obtained on large Optipore resins earlier [4]. Due to the enormous pressure drop when the column is packed with small resins, it is not possible to perform this experiment with the grinded resins.

The effective diffusion coefficient $\left(D_{\text {eff }}\right)$ was derived from these experiments by fitting the data with the homogeneous solid diffusion method (HSDM, Equation 1) [11, 12]. This method is described in more detail elsewhere [9].

$\frac{q_{t}}{q_{\mathrm{eq}}}=1-\frac{6}{\pi^{2}} \sum_{n=1}^{n} \frac{1}{n^{2}} \exp \left\{-\frac{4 \cdot D_{\mathrm{eff}} \cdot n^{2} \cdot \pi^{2}}{d^{2}} \cdot t\right\}$

With $\mathrm{q}_{\mathrm{t}}$ the solute loading on the resin $\left[\mathrm{g}_{\text {solute }} / \mathrm{g}_{\mathrm{resin}}\right]$ at time $\mathrm{t}[\mathrm{s}]$ and $\mathrm{q}_{\mathrm{eq}}$ the solute loading at equilibrium, $n$ the series of integers, $D_{\text {eff }}\left[\mathrm{m}^{2} / \mathrm{s}\right]$ the effective diffusion coefficient and $d[\mathrm{~m}]$ the diameter of the resin. With the sum of least squares the ZLC adsorption data are fitted with Equation 1 to extract $D_{\text {eff }}$.

\section{Breakthrough curves MMMs}

The breakthrough curves for HMF adsorption on MMMs were determined with a set up shown in Figure 2. 7 MMMs were packed in a membrane filter holder. The MMMs were covered with a paper spacer to ensure an even distribution of the solution over the membranes. The breakthrough curves were determined with at a flow rate of $8,16,32$ and 100 bed volumes per hour (BV/h) with HMF (5000 mg/l). $5000 \mathrm{mg} / \mathrm{l} \mathrm{HMF} \mathrm{solution} \mathrm{was} \mathrm{used} \mathrm{to} \mathrm{be} \mathrm{able} \mathrm{to} \mathrm{compare} \mathrm{the} \mathrm{data} \mathrm{with} \mathrm{the} \mathrm{data}$ obtained for a packed bed with Optipore resins $(557 \mu \mathrm{m})$ in previous research [4]. All experiments were conducted at $20^{\circ} \mathrm{C}$. The outflow of the column was fractionated in fractions with a volume of $0.5 \mathrm{BVs}$. The concentration of every other fraction was analyzed with UV-VIS at $284 \mathrm{~nm}$. 
The HMF MMM capacity (\%) was presented as the HMF adsorption capacity at $100 \%$ breakthrough of the inlet concentration $\left(\mathrm{q}_{100 \% \text { breakthrough }}\right)$ (determined by measuring the surface area above the breakthrough curve from 0 BV to $100 \%$ breakthrough) over the equilibrium adsorption capacity (Equation 2).

HMF MMM capacity $(\%)=\frac{q_{100 \% \text { breakthrou gh }}}{q_{\text {eq }}} \cdot 100 \%$

The HMF MMM adsorption efficiency (\%) was presented as the HMF adsorption capacity at $10 \%$ breakthrough of the inlet concentration $\left(\mathrm{q}_{100 \% \text { breakthrough }}\right)$ (determined by measuring the surface area above the breakthrough curve from $0 \mathrm{BV}$ to $10 \%$ breakthrough) to the column capacity (Equation 3).

HMF MMM efficiency (\%) $=\frac{q_{10 \% \text { breakthrou gh }}}{q_{100 \% \text { breakthrou gh }}} \cdot 100 \%$

\subsection{Results and Discussion}

\subsubsection{Adsorbent analysis}

Particle size measurement and nitrogen adsorption of grinded Optipore

The grinded Optipore resins were analyzed with DLS and nitrogen adsorption to determine the average particle size and BET surface area. The results are given in Table 1.

Table 1: Properties Optipore resins.

Large resins [3] Grinded resins

Particle size diameter $[\mu \mathrm{m}] \quad 557$

$\begin{array}{llr}\text { BET surface area }\left[\mathrm{m}^{2} / \mathrm{g}_{\mathrm{resin}}\right] & 1321 \pm 2 & 1125 \pm 1.5\end{array}$

The average particle size of the grinded resins is $15 \mu \mathrm{m}$ which is smaller than the size of the sieve.

This is probably caused by agglomeration of the grinded particles during sieving [9]. The BET 
surface area of the grinded resins is slightly smaller than that of the ungrinded particles [3]. Grinding of the resins causes blockage of some of the of the resin pores, thereby reducing the BET surface area.

SEM analysis of the MMMs

The SEM micrographs of the cross sections and top surfaces of membranes 1-3 are given in Figure 1.

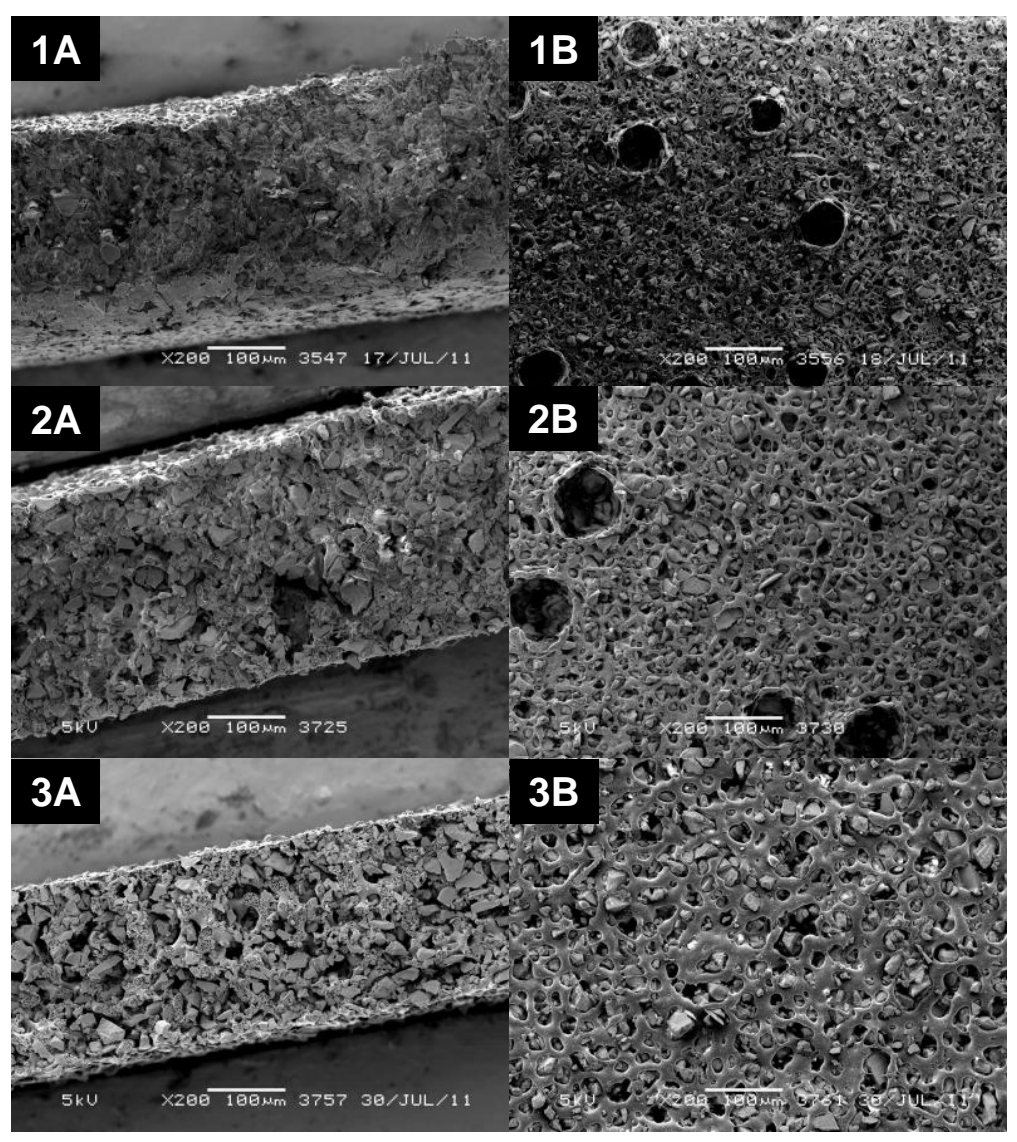

Figure 1: SEM micrographs of Optipore MMMs; cross section and top surface of the membrane prepared with $50 \mathrm{~g}$ NMP: 1a,b; 60 g NMP: 2a,b; 75 g NMP: 3a,b. a. All magnifications: 200x.

The SEM micrograph of the cross section of membrane 1 (Figure 1a) shows a very dense structure. The pores of the membrane are small and not very well interconnected. Furthermore the adsorption particles appear to be covered by the polymer. The top surface of the membrane (Figure $1 \mathrm{~b}$ ) shows some craters caused by air bubbles trapped in the solution during the casting process. The dense structure of the membrane as well as the entrapment of gas is probably 
caused by the high viscosity of the casting solution. Most likely, the high viscosity is due to swelling of the hypercrosslinked Optipore, thus removing NMP from the solution $[3,10,13]$.

The membranes prepared from a solution with a higher amount of NMP (membrane 2: $60 \mathrm{~g}$ NMP; membrane 3: $75 \mathrm{~g} \mathrm{NMP}$ ) and consequently a lower viscosity show a more porous homogeneous morphology. Although some craters and macrovoids are still visible (Figure 2a,b), the particles are well embedded, but uncovered and highly accessible. Addition of an extra $5 \mathrm{wt} \%$ NMP (Figure $3 a, b)$ resulted in excellent MMMs with highly interconnected pores and accessible adsorption particles. Furthermore the surface did not show craters. Membrane 3 was used for further adsorption and kinetic experiments.

\subsubsection{Adsorption isotherms}

Figure 2 shows the adsorption isotherms of the large and the grinded resins as well as those of the MMMs, normalized for the wt\% resins in the membrane. Adsorption of HMF on native PES was not observed (not shown). The adsorption capacity data of the large resins are obtained from previous research [3].

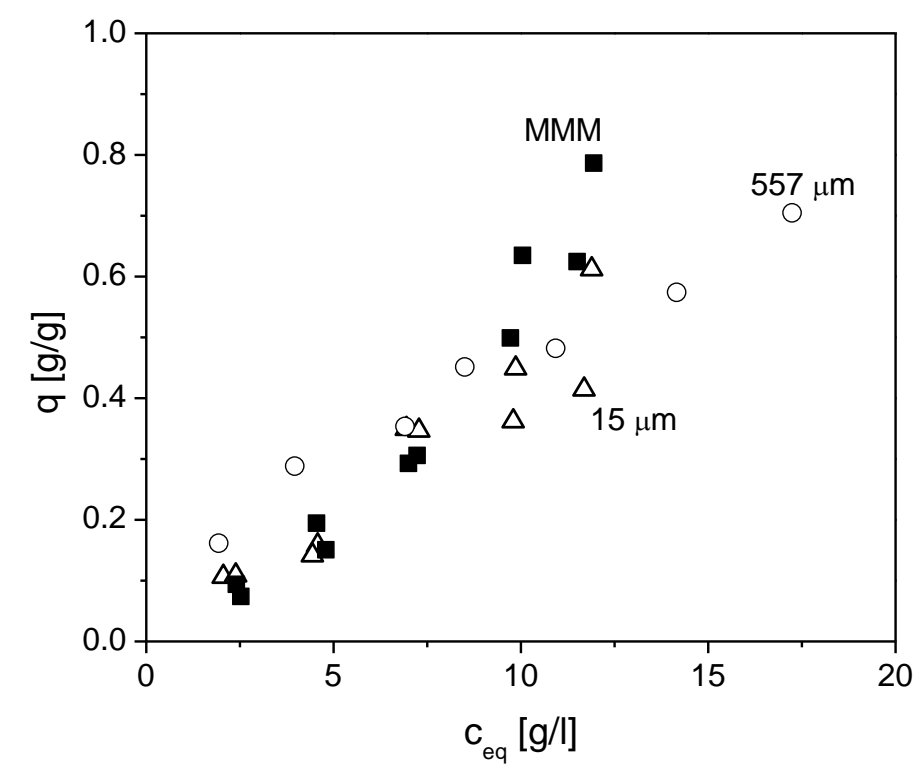

Figure 2: $\mathrm{HMF}$ adsorption isotherms ([ $\left.\left.\mathrm{C}_{0}\right]=3-30 \mathrm{~g} / \mathrm{l}\right) ; \bigcirc 557 \mu \mathrm{m}$ resins [3], $\triangle: 15 \mu \mathrm{m}$ resins, $\mathbf{\Perp}: \mathrm{MMM}$ with $15 \mu \mathrm{m}$ resins.

Figure 2 shows that the adsorption capacity of the large and the grinded resins is comparable. The reduced BET surface area of the grinded resins does not lead to a lower adsorption capacity. This 
indicates that only small pores are blocked after the grinding. The adsorption isotherm of the MMM also shows that incorporation of the grinded particles into the membrane does not lead a reduction in adsorption capacity. This is proof that the adsorption particles are indeed very well accessible, as was observed in the SEM micrographs.

\subsubsection{Kinetic analysis}

Zero length column experiments

The results of the ZLC experiments of HMF in large Optipore resins and small resins incorporated in MMMs are shown in Figure 3. The ZLC data of the large resins are obtained from previous research [4]. $D_{\text {eff }}$ was determined from a fit of the data with Equation 1.

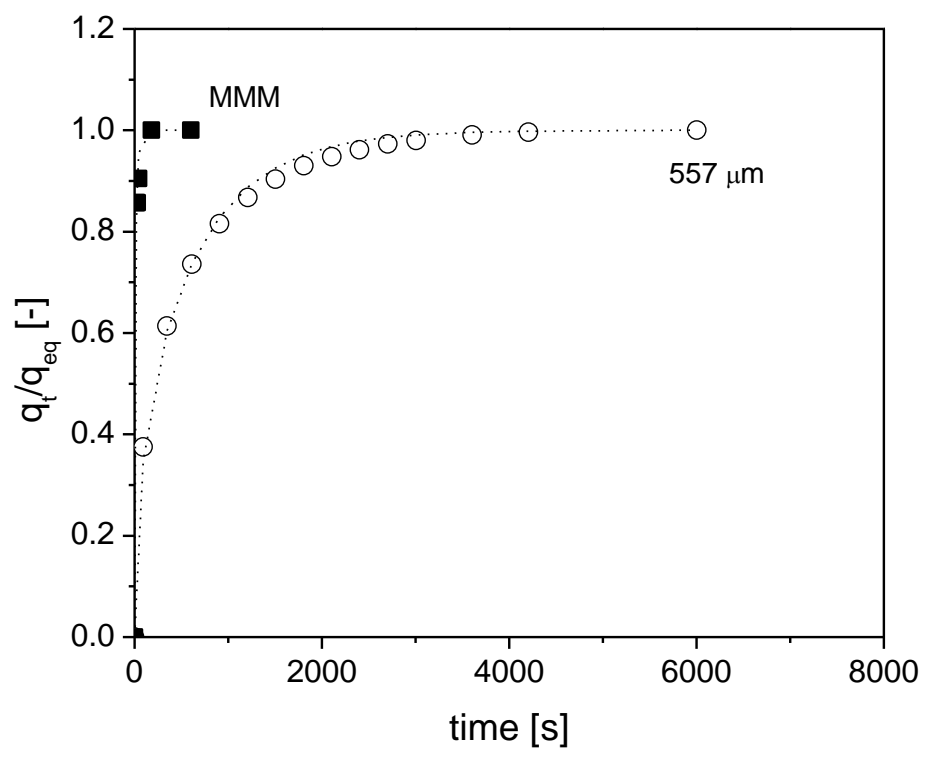

Figure 3: Relative HMF loading [q/ $/ \mathrm{q}_{\mathrm{eq}}$ ] in time; $\bigcirc 557 \mu \mathrm{m}$ resins [4], $\mathbf{m}: \mathrm{MMM}$ with $15 \mu \mathrm{m}$ resins. The dotted line represent the relative HMF adsorption calculated with the HSDM.

Figure 3 shows that the adsorption equilibrium in the MMMs is reached within 3 minutes while it takes approximately 100 minutes to reach adsorption equilibrium in the large resins. Earlier work showed that at these flow rates external mass transfer through the liquid boundary for the large resins is not limiting the adsorption rate inside the particle and can consequently be neglected [4]. It is therefore allowed to use the simplified model without the liquid boundary layer which results in a value of $D_{\text {eff }}$ for the large particles of $1.110^{-11} \mathrm{~m}^{2} / \mathrm{s}$, which is comparable to the value of $8.010^{-12}$ 
$\mathrm{m} / \mathrm{s}$, that is obtained when the more complex model that includes the liquid boundary layer is used

[4]. The $D_{\text {eff }}$ for the MMMs determined by the same methodology is $3.3 \cdot 10^{-13} \mathrm{~m} / \mathrm{s}$. Incorporation of the resins in the MMMs resulted in a $\sim 30$ times reduction in $D_{\text {eff. }}$ Literature shows that the diffusion coefficient is independent of the particle radius $[14,15]$. Consequently, when small resins are incorporated into MMMs, ideally the effective diffusion coefficient should be equal to that of the large resins. Based on the BET surface analysis (Table 1) the small and large particles are not comparable, as they show different surface areas. However the adsorption isotherms (Figure 2) show that this did not alter the adsorption capacity of the grinded resins for HMF and we therefore assume that the pores of the resins that adsorb HMF are relatively comparable.

When the pure resins and MMMs were compared in earlier research [9], the apparent diffusion coefficient of the resins was reduced 60 to 200 times when the resins were grinded and incorporated in the MMMs. This was most likely due to an increased path length created by the pores of the MMMs surrounding the particles [9]. With this new MMM the reduction of the effective diffusion coefficient compared to the pure resins is only 30 times, which is a large improvement. It is possible that further development of the MMMs could bring both diffusion coefficients closer together. However, due to the nature of membranes, including dead end pores, we do not believe that it is possible to fabricate membranes that will show identical $D_{\text {eff }}$ to the pure resins. In MMMs some pore blocking of the resins, or lengthened path for diffusion will always exist.

\section{Breakthrough curves}

Figure 4 shows a breakthrough curve $(8 \mathrm{BV} / \mathrm{h})$ of the $\mathrm{MMM}$ and its reproducibility. The entire volume of the MMM is used as a bed volume and the value is not compensated for the PES membrane and its porosity. 


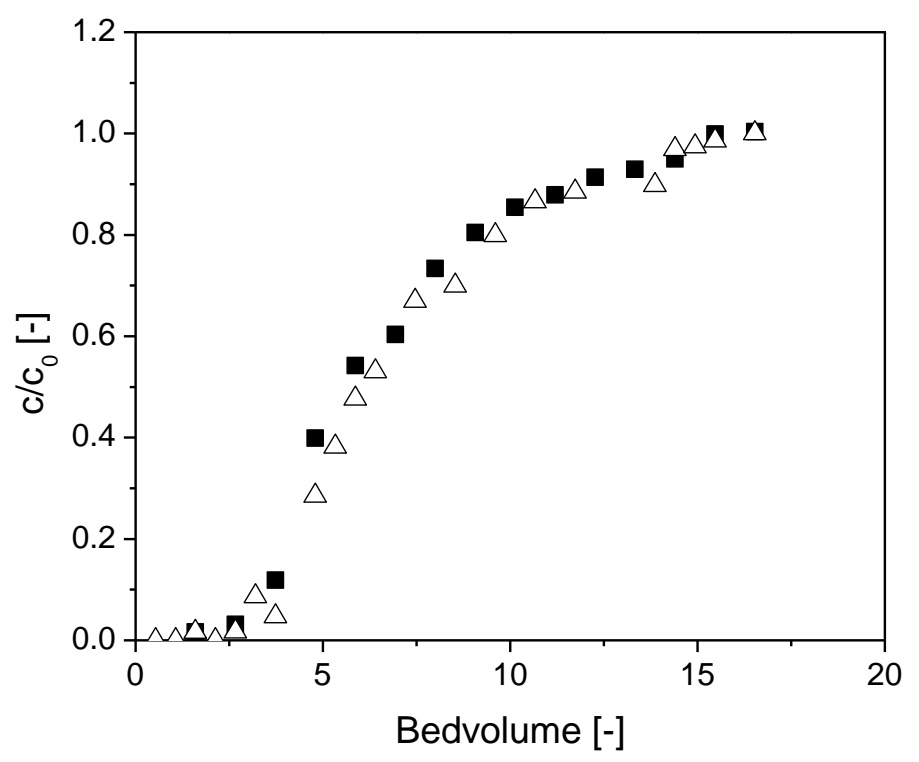

Figure 4: HMF breakthrough curves of the MMM at $20^{\circ} \mathrm{C}, 5000 \mathrm{mg} / \mathrm{HMF}, 8 \mathrm{BV} / \mathrm{h}$ : Experiment 1; $\triangle$ Experiment 2.

Both curves almost overlap and show comparable breakthrough points, showing the reproducibility of the measurements.

Figure 5 shows the breakthrough curve of the MMMs performed at different bed volumes. Again the data are not corrected for the PES membrane or its porosity.

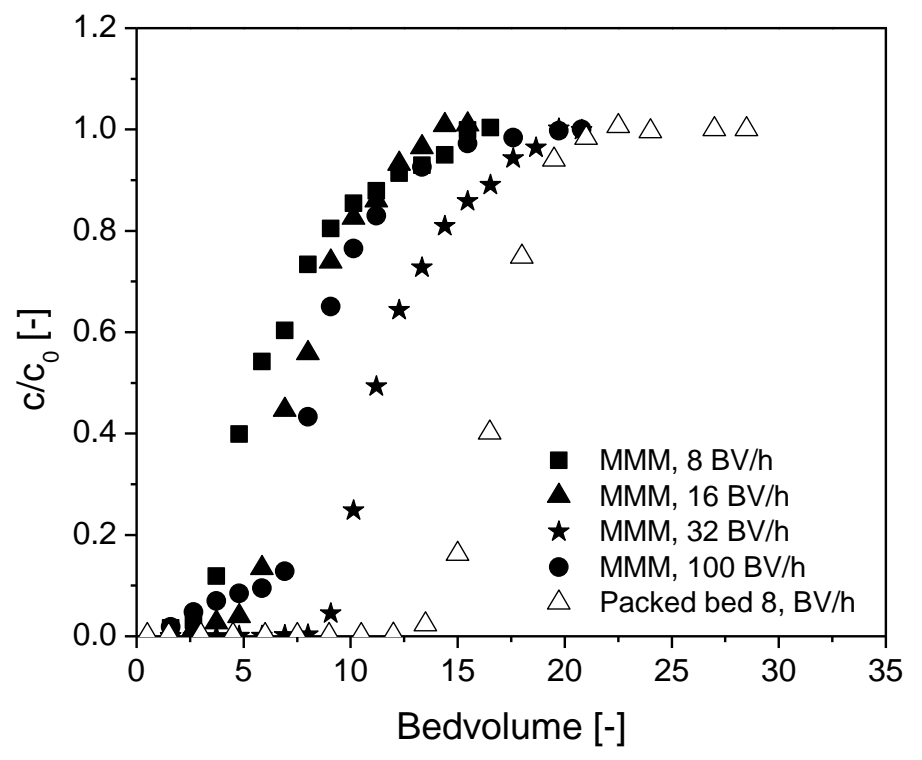

Figure 5: $\mathrm{HMF}$ breakthrough curves at $20^{\circ} \mathrm{C}$ of the $\operatorname{MMMs}(8,16,32,100 \mathrm{BV} / \mathrm{h})$ and packed bed $(8 \mathrm{BV} / \mathrm{h})[4]$ 
Figure 5 shows that breakthrough of the $8 \mathrm{BV} / \mathrm{h}$ curve is early ( 1.5 BV) and a significant tailing occurs. When the flow rate is increased to 16 and $32 \mathrm{BV} / \mathrm{h}$ respectively, breakthrough occurs later (at $\sim 3$ and $\sim 9$ BV respectively) and tailing is reduced slightly. When the flow rate is further increased to $100 \mathrm{BV} / \mathrm{h}$, breakthrough is earlier ( 2.5) and between 0 and $7.5 \mathrm{BV}$ a typical flow through pattern of HMF is observed, indicating that the flow rate is too high to allow sufficient time for HMF adsorption. The better adsorption performance at higher flow rates shows that the external resistance to adsorption is improved at higher flow rates. The importance of the external resistance in this experiment is in contrast with its small importance during the ZLC experiments, were the external resistance only shows a relatively small contribution. The difference is explained by the low flow rates in the breakthrough experiments. Internal pore adsorption is so fast that external adsorption becomes limiting. This effect is reduced at higher flow rates. Based on these experiments we cannot distinguish whether this is due to increased flow around the particles, thereby increasing turbulence and reducing the film layer around the particles, or due to improved flow through the pores of the membrane or a combination of both. Previously performed experiments with a packed bed containing Optipore resins did not show any improvement when the flow rate was increased [4]. Overall MMMs can handle of much higher flow rates and therefore higher throughputs without loss of adsorption performance. When the performance of the Optipore MMMs is compared to that of the packed bed with Optipore (Figure 5), HMF breakthrough is still earlier. This is caused by the much lower Optipore volume in the MMMs than in the packed bed. The total capacity of the MMMs is therefore lower than that of the packed bed.

To take this into account, the data of the MMMs are corrected for the actual amount of resin in the MMM. The data are presented in Figure 6. When the only the resin volume in the MMM is considered the resins in the MMMs perform much better than in the packed bed (which is also corrected for resin volume). Breakthrough is later while flow rates can be much higher which even increases the adsorption capacity. This is also reflected in column capacity expressed as percentage of the equilibrium concentration (Table 2). The MMMs have a much higher column capacity than the packed bed and it increases with increasing flow rate. The MMMs on the other hand do show more tailing than the packed bed. This is likely due to axial dispersion that is observed because the bed length of MMMs is short. Lou and Fried [16] suggested that for good adsorption performance of adsorption membranes the non-uniformity in porosity and thickness 
needs to be levelled out by using a stack of at least 30 membranes. Avramescu et al. [17] investigated the breakthrough curve of a stack of 5, 10 and 15 flat sheet MMMs and showed that the adsorption capacity and breakthrough times were almost proportional to the amount of membranes and the curves were steep for all stacks, indicating that a stack of 5 membranes can suffice. Avramescu et al. [18] also investigated the effect of stacking fibers in series and found that that addition of even one fiber improved the shape of breakthrough behavior enormously up to a series of 5 fibers. Breakthrough occurred relatively later and the curve was steeper. We therefore believe that, although our membranes already show excellent adsorption behavior, it is possible to improve the breakthrough curve of the Optipore MMMs for HMF adsorption even more by adding extra layers of MMMs.

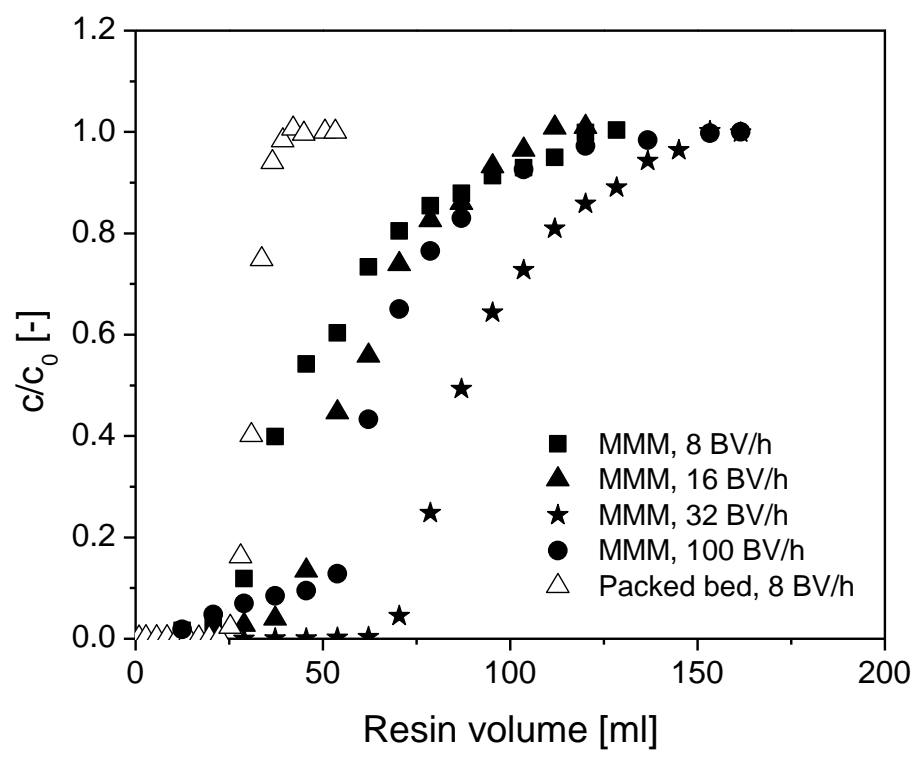

Figure 6: $\mathrm{HMF}$ breakthrough curves at $20^{\circ} \mathrm{C}$ of the MMMs $(8,16,32,100 \mathrm{BV} / \mathrm{h})$ and packed bed $(8 \mathrm{BV} / \mathrm{h})$ normalized for the resin volume. 
Flow rate

$[\mathrm{BV} / \mathrm{h}]$

\begin{tabular}{lccc}
\hline MMM & 8 & 86 & 55 \\
& 16 & 102 & 69 \\
& 32 & 157 & 77 \\
& 100 & 114 & 69 \\
Packed bed [4] & 8 & 52 & 87
\end{tabular}

Column capacity

[\%]

\section{Column efficiency}

[\%]

The breakthrough graphs show that the adsorption performance of MMMs is improved significantly by using higher flow rates. For the separation of HMF from glucose, Optipore in a conventional packed bed already performs very well and the use of MMMs is not persé beneficial. The higher applicable throughput in MMMs is compensated by a lower absolute capacity of the MMMs compared to a conventional packed bed. Based on the used flow rates and determined breakthrough points in this research, the total volume of the MMM adsorption unit could be $\sim 3$ times smaller than the packed bed adsorption unit for removing $5 \mathrm{~g} / \mathrm{l} \mathrm{HMF}$ when desorption is not taken in account. However the operating parameters of the packed bed and the MMMs have not been optimized. Therefore conclusions on which system would be best require further research and a thorough comparison of capital and operating cost of both systems based on e.g. concentration, flow rate, breakthrough points, pressure drops, mechanical strength, desorption, maintenance and life time expectancy. However these preliminary results show that MMMs could be very beneficial for small molecule adsorption.

We would like to emphasize that earlier research of Optipore and several other resins for the removal of furans as well as a comparison with adsorbents available in literature (e.g. ion exchange resins, XAD4, activated carbon, zeolites), showed that Optipore outperforms every other adsorbent investigated for furan adsorption so far $[3,4]$. We believe that most adsorbents, either for furans or other small solutes, do not show such outstanding performance and as such would benefit much more from incorporation into MMMs. We therefore strongly advice to investigate MMMs further, 
also for poor performing adsorbents, to examine its economic potential for high throughput of small molecule adsorbing systems.

\subsection{Conclusion}

The newly fabricated Optipore MMMs show the same excellent HMF adsorption capacities as the large resins and the grinded resins. This shows that the fabrication method of the MMMs does not block adsorption sites and allows for the full use of the particle capacity. ZLC experiments show that the effective diffusion coefficient is only slightly reduced when particles are incorporated in the membranes, while the overall adsorption time is improved enormously. This is reflected in the fast flow rates that could be used when the breakthrough curves were determined. The breakthrough curves also show that the adsorption capacity of the resins themselves is even improved when the membranes are used compared to a packed bed with large resins.

Due to the open structure of the MMMs, the MMMs show improved adsorption capacity and fast adsorption rates at high flow rates, which allows for high throughput application of MMMs for HMF separation. Furthermore this research shows that MMMs could be a good alternative for traditional packed beds for the adsorption of small molecules.

\subsection{Acknowledgment}

This work was carried out within project SC-00-04 for the Institute for Sustainable Process Technology (ISPT), The Netherlands

\subsection{References}

1. Huang, H.J., et al., A review of separation technologies in current and future biorefineries. Separation and Purification Technology, 2008. 62(1): p. 1-21.

2. Mills, T.Y., N.R. Sandoval, and R.T. Gill, Cellulosic hydrolysate toxicity and tolerance mechanisms in Escherichia coli. Biotechnology for Biofuels, 2009. 2: p. 1-11.

3. IJzer, A.C., et al., Performance analysis of aromatic adsorptive resins for the effective removal of furan derivatives from glucose. Journal of Chemical Technology and Biotechnology, 2014. 90(1): p. 101-109.

4. IJzer, A.C., et al., Adsorption kinetics of Dowex ${ }^{T M}$ Optipore ${ }^{T M}$ L493 for the removal of the furan 5-hydroxymethylfurfural from sugar. Journal of Chemical Technology \& Biotechnology, 2015.

5. Avramescu, M.-E., Z. Borneman, and M. Wessling, Membrane chromatography, in Handbook of Membrane Separations; Chemical, Pharmaceutical, Food and Biotechnological Applications, A.K. Pabby, S.S.H. Rizvi, and A.M. Sastre, Editors. 2008, CRC Press. 
6. Ghosh, R., Protein separation using membrane chromatography: Oportunities and challenges. Journal of Chromatography A, 2002. 952(1-2): p. 13-27.

7. Orr, V., et al., Recent advances in bioprocessing application of membrane chromatography. Biotechnology Journal, 2013. 31(4): p. 450-465.

8. Saiful, Mixed matrix membrane adsorbers for protein and blood purification, 2007, University of Twente: Enschede.

9. IJzer, A.C., et al., Very fast adsorption of biological anions by particle loaded mixed matrix membranes. Submitted, 2015.

10. Borneman, Z., Particle loaded membrane chromatography, in Ph.D. thesis2006, University of Twente: Enschede.

11. Ruthven, D.M., Principles of Adsorption \& Adsorption Processes. 1984, United States of America: John Wiley \& Sons, Inc.

12. Karger, J., D.M. Ruthven, and D.I. Theodorou, Diffusion in Nanoporous Materials. 2011, Weinheim: Wiley-VCH.

13. Tsyurupa, M.P. and V.A. Davankov, Porous stucture of hypercrosslinked polystyrene: State-of-the-art mini-review. Reactive and Functional Polymers, 2006. 66(7): p. 678-779.

14. Djekic, T., Effective intraparticle diffusion coefficients of $\mathrm{CoCl}_{2}$ in mesoporous functionalized silica adsorbents. Adsorption, 2007. 13(3-4): p. 231-237.

15. Schouten, N., et al., Kinetic analysis of anionic surfactant adsorption from aqeous solution onto activated carbon and layered double hydroxide with the zero length column method. Separation and Purification Technology, 2009. 68(2): p. 199-207.

16. Liu, H.-C. and J.R. Fried, Breakthrough of lysozyme through an affinity membrane of cellulose-ciacron blue. AIChE, 1994. 40(1): p. 40-49.

17. Avramescu, M.-E., Z. Borneman, and M. Wessling, Dynamic behavior of adsorber membranes for protein recovery. Biotechnology and Bioengineering, 2003. 84(5): p. 564572.

18. Avramescu, M.-E., Z. Borneman, and M. Wessling, Particle-loaded hollow-fiber membrane adsorbers for lysozyme separation. Journal of Membrane Science, 2008. 322(2): p. 306313. 


\section{Reaction media for molecularly imprinted membranes for acid adsorption applications}

\subsection{Abstract}

In biorefinery processes biomass is converted to produce fuels, power, heat and value-added chemicals. Further processing and/or legislation demands often require removal of process impurities present in ppm concentrations from the products. Especially interesting in this respect are molecular imprinted membranes as a method to remove trace amounts of e.g. acids from a biobased mixture at a high degree of selectivity. Molecular imprinting is a technique to create adsorbers with tailored adsorption sites for specific molecules. In this work, we show the successful preparation of molecularly imprinted membranes for the selective adsorption of acids. Especially, the effect of the solvent during imprint formation has been investigated. The results show that careful selection of the solvent and $\mathrm{pH}$ is essential for successful molecular imprinting and recognition. The optimal conditions for complex formation have been determined by the continuous variation method. For the selective adsorption of salicylic acids with molecular imprinted membranes, the results show imprinting can be achieved at low $\mathrm{pH}$ in a solvent that mimics the aqueous environment. Molecular imprinting with a high crosslinker to polymer ratio showed to alter the $\mathrm{pH}$ inside the membrane and consequently the charge inside the membrane and its adsorption behavior. The membranes investigated show clear $\mathrm{pH}$ transitions and fast kinetics. Consequently, molecular imprinting of membranes (MIMs) is a very interesting technology for the selective recovery or separation of specific molecules from complex feed mixtures.

\subsection{Introduction}

In biorefinery processes biomass is converted to produce fuels, power, heat and value-added chemicals. Further processing and/or legislation demands often require removal of process impurities present in ppm concentrations from the products. Among the interesting molecules produced in biorefinery processes, are acids (e.g. for pharmaceuticals, food, cosmetics, and as 
base chemical for plastics, preservatives etc.). Conventional separation technologies are energy intensive and require thermal stability of the molecules (e.g. distillation) or require long bed volumes with corresponding high pressure drops (e.g. (ion exchange) chromatography) [1]. Molecular imprinting has been recognized as an alternative for the selective separation of acids from complex mixtures.

Molecular imprinting of polymers is a technique to create adsorbers with tailored adsorption sites for specific molecules. This makes these molecularly imprinted polymers (MIPs) comparable with biological processes such as ligand-receptor binding. Although molecular imprinting of acidic molecules is considered challenging [2], MIPs have successfully been prepared for biobased acid recognition and selective separation. MIPs are for instance used for the separation of phenolic acids from the cinnamic and the p-hydroxybenzoic group [3], removal of acidic herbicides from water [2], recognition and adsorption of acidic pharmaceuticals such as aspirin [4] and naproxen [5, 6], and the recognition of the acidic amino acids aspartic acid [7, 8] and glutamic acid [5-7].

MIPs are polymers that contain functional groups, which are processed or polymerized in the presence of a template molecule (e.g. the targeted acid) (Figure 1) [8]. The polymer binding site, formed by these specific functional groups, is ordered in a three dimensional structure that makes the polymer also shape selective. The size and shape of the template molecule are mirrored in the size and shape of the binding site and the template fits exactly in the adsorption site of the polymer. A deviation of the molecular structure from the target molecule, such as an altered place of the molecules functional group, substitution of the templates functional group by another functional group or addition of a bulky group, hinders adsorption. This mechanism makes it possible to separate almost identical molecules from each other by adsorption with molecularly imprinted polymers. 
1

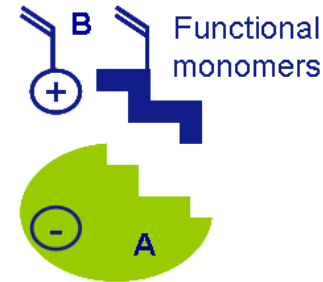

Target molecule
Self assembly
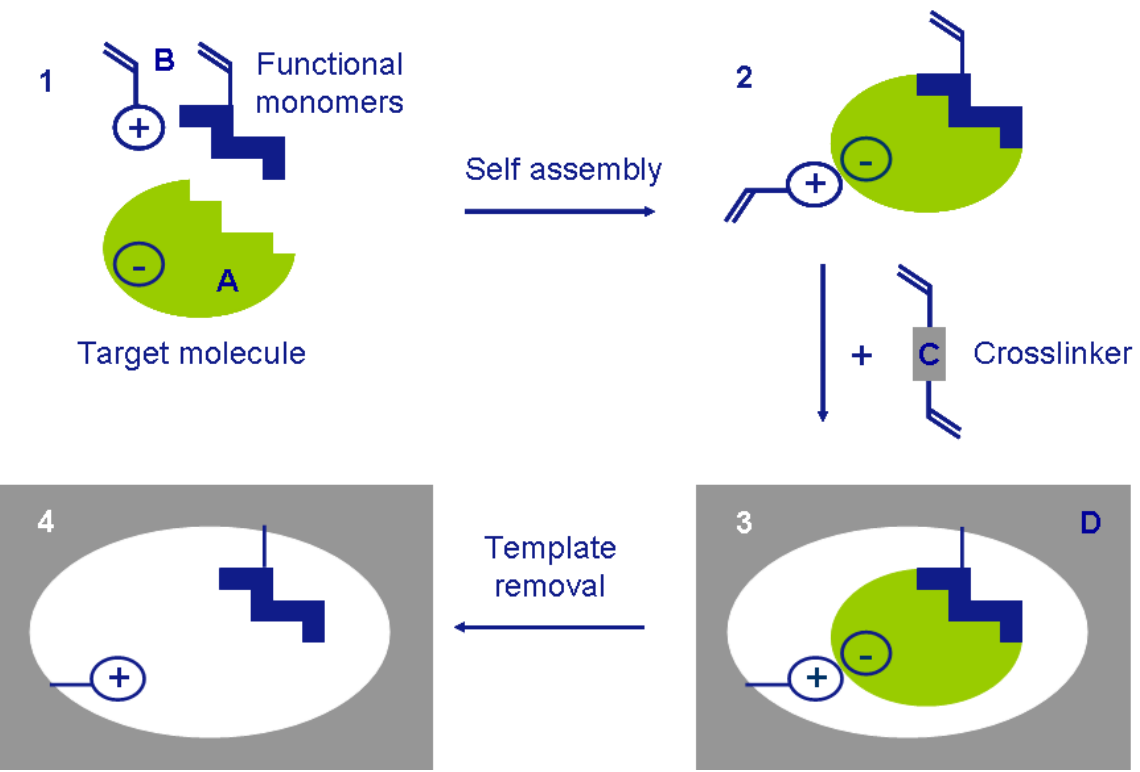

Template removal

Figure 1: Illustration of the molecular imprinting process: 1: mixing of target molecule or template and reactants; 2: selfassembling of target molecule and reactants; 3: stabilization of target molecule by crosslinking; 4: extraction of target molecule or template finally resulting in a polymer with shape and size selective binding site (MIP).

The general method to make an imprint in a polymer is by polymerization of functional monomers and crosslinking of the monomers in the presence of the template molecule (the target molecule), after which the polymers are grinded into particles [9]. In order to preserve the three-dimensional ordering of the functional groups, MIPs are prepared with a high crosslinker-to-functional monomer ratio [8]. This results in a very rigid polymer network with limited accessibility of the target molecule to the imprinted sites, other than those at the surface of the polymer $[14,15]$. The adsorption and desorption of the template deeper into the MIP layer is therefore limited by diffusion through the small cavities formed by the imprinted sites of the MIPs outer layer. This problem can be solved by increasing the surface-to-volume ratio of the imprinted polymer by coating of very thin layers of MIP on the walls of the pores of microporous membranes to form so-called molecularly imprinted membranes (MIMs)) [10]. Fast adsorption kinetics created by the high surface-to-volume ratio of the MIP layer combined with the high porosity of the membrane bed allow fast solute throughput with low pressure drops. Consequently, bed volumes can be smaller when compared to conventional packed beds. Schneider et al. [11] showed that regularly fabricated MIP particles have lower capacity and selectivity than comparable MIMs. With its relatively high capacity, 
selectivity and low pressure drop, MIMs could be the ultimate solution for selective solid phase extraction of a specific solute from a liquid.

Formation of the complex between template and functional monomer and its effectiveness in the application depends on the type of functional groups and the nature of the reaction medium, as these determine the number and the type of bonds formed (van der Waals, hydrogen bonding, ionic interactions, hydrophobic forces) and therefore the shape, strength and stability of the 3D complex structure. Generally apolar solvents with low dielectric properties, such as toluene and chloroform are used for molecular imprinting [12] to allow strong hydrogen bonding. Polar aprotic solvents, such as acetonitrile, are also often used [12]. They still allow hydrogen bonding, but interfere with van der Waals forces and ionic interactions. Protic solvents, such as water and methanol, allow ionic interactions, but because of their high dielectric constants, interfere with hydrogen interactions and are therefore less often used [2, 12]. However, good recognition and adsorption of acids in MIMs in aqueous applications requires that the bonds formed during imprinting in the solvent are similar to the bonds formed during application, which makes protic solvents better candidates for molecular imprinting for aqueous applications. Ionic interactions are more stable than hydrogen interactions since the latter are easily destabilized by hydrogen bond formation with water.

Here we investigate the use of MIMs for biorefinery applications. This requires recognition properties in aqueous environments. Considering the importance of the type of solvent, especially the tuning of solvent conditions (composition, $\mathrm{pH}$ ) during imprinting to mimic such aqueous applications and to induce the formation of ionic bonds during imprinting, is the focus of this research. As such, this approach shows the impact of the solvent conditions on the formation of MIMs, the potential of effective MIM formation and its application in biorefinery concepts.

\subsection{Theory}

Molecular imprinting of acids for aqueous applications is usually performed with alkaline functional monomers, such as 4-vinylpyridine (4VP), vinylimidazole or aminostyrene [13]. In this research 4VP is used because 4VP molecularly imprinted polymers are known to show better chiral 
separation than vinylimidazole [15]. Furthermore, the UV crosslinking of 4VP on membranes has been described by Donato et al. [14]. To ensure that ionic interactions are studied and not hydrogen interactions, salicylic acid ( $\mathrm{SaA}$ ) is chosen as the target molecule (Figure 2). SaA is an excellent model target molecule because it can only form a bond with another molecule (complex) with acid-base interactions when the molecule is deprotonated [16, 17] (Figure 2c). This is caused by the tendency of the molecule to form intra-molecular hydrogen bonds between the hydroxyl group and the acid group (Figure $2 \mathrm{~b}$ ) rather than inter-molecular hydrogen bonds of these groups with another molecule, such as a functional monomer [17, 18]. Molecular imprinting based on hydrogen bonding is therefore unlikely when SaA is uncharged, as has been demonstrated by the absence of a molecular imprint in aqueous environment by Park et al. [18].

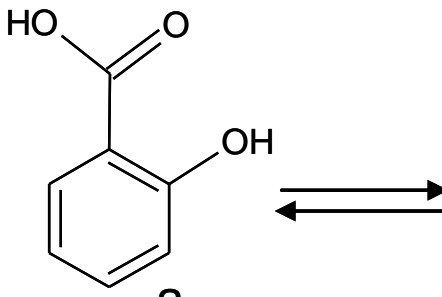

a<smiles>O=C(O)c1ccccc1O</smiles>

b

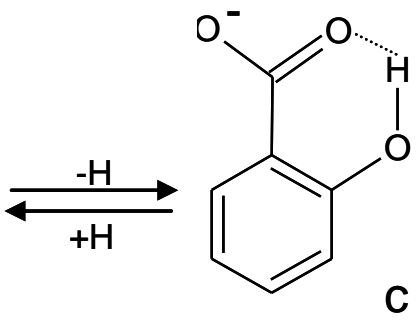

Figure 2: Structures of SaA. (a) no bond (b) intra-molecular hydrogen bond; (c) deprotonated with intramolecular hydrogen bond

The deprotonation of SaA is determined by its pKa. In water the pKa of SaA and 4VP is 2.9 and 5.9 respectively and the $\mathrm{pH}$ should be maintained between these two values to achieve ionic interaction. Unfortunately molecular imprinting with $4 \mathrm{VP}$ is not possible in water because 4VP autopolymerizes at low concentrations. In methanol 4VP and the used crosslinker diethyleneglycoldimethacrylatre (DEGDM) are soluble and stable, but the pKa of SaA in methanol is higher than the pKa of 4VP. Roses et al. [23, 24] showed that the pKa of acids and bases is dependent on the composition of the solvent. The presence of an organic solvent such as methanol or acetonitrile in water increases the pKa of an acid and lowers the pKa of a base. The relation between the pKa of $\mathrm{SaA}$ and $4 \mathrm{VP}$ and the corresponding concentration of methanol in water is derived from this research and graphically visualized in Figure 3. 


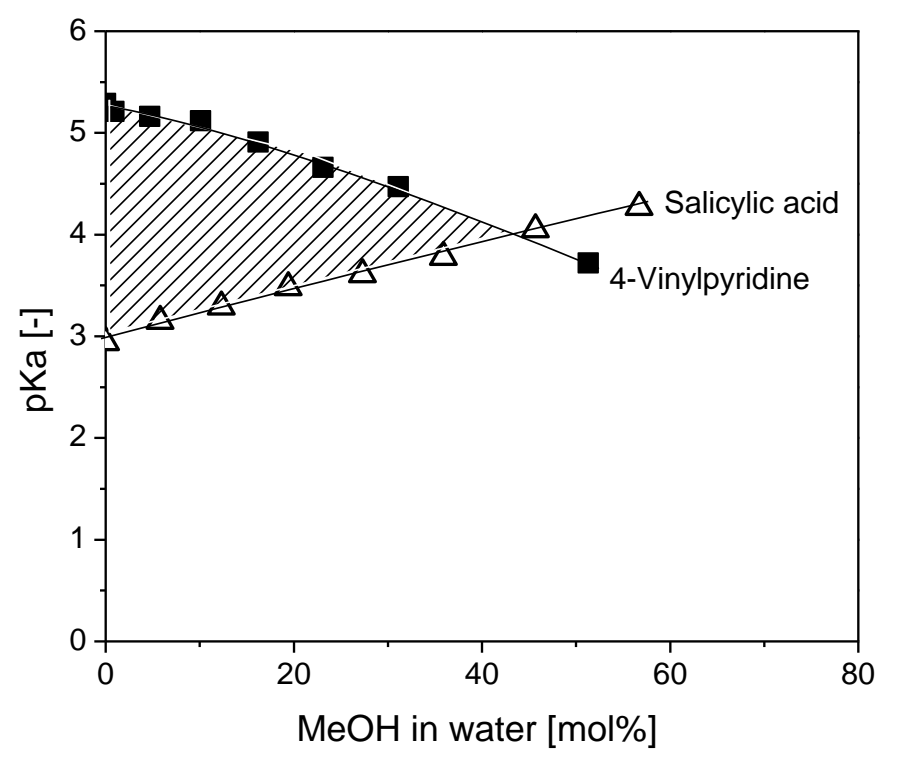

Figure 3: pKa of $4 \mathrm{VP}$ and $\mathrm{SaA}$ as function of concentration methanol in water (mol\%) calculated based on research of Roses et al. [19, 20].

Figure 3 shows that as the methanol content in water increases, the pKa of 4VP decreases and the pKa of SaA increases. When the methanol in water content is $42 \mathrm{~mol} \%$, the pKa of SaA and 4VP are equal, increasing the methanol content further leads to a higher pKa of SaA than that of 4VP. The shaded area in Figure 3 indicates the $\mathrm{pH}$ and solvent composition where 4VP is protonated and $\mathrm{SaA}$ is deprotonated and consequently the desired ionic interaction between $\mathrm{SaA}$ and $4 \mathrm{VP}$ is possible. Since molecular imprinting of SaA is only possible when it is deprotonated, the presence of a molecularly imprinted based adsorption selectivity of the membranes for SaA will therefore demonstrate that an ionic interaction between $\mathrm{SaA}$ and functional monomer in the reaction mixture (before the polymerization) occurred. 


\subsection{Materials and methods}

\subsubsection{Materials}

Porous commercial polypropylene (PP) membranes (Accurel 2E-HF) were purchased from Membrana GmbH. Salicylic acid (SaA), 4-hydroxybenzoic acid (4-HBA), 3-methylsalicylic acid (3MSaA), 4-vinylpyridine (4VP), pyridine, diethyleneglycoldimethacrylate (DEGDM), bezoïn ethyl ether (BEE), sodium phosphate monobasic monohydrate (SPM), sodium phosphate dibasic anhydrous (SPD), phosphoric acid (85\%), acetic acid, sodium acetate and sulfuric acid were purchased from Sigma Aldrich and used as received. Lactic acid (LA, 100\%) was kindly provided by Purac (Gorinchem, The Netherlands). Ethanol and methanol were of analytical grade and obtained from Merck.

\subsubsection{Complex formation and stoichiometry}

The complex formation between $\mathrm{SaA}$ and pyridine in different solvent compositions was investigated by UV-Vis using the continuous variation method [21]. $410^{-5} \mathrm{M}$ pyridine (Pyr) and SaA solutions were prepared in solvents according to Table 1. SaA was mixed with Pyr at a SaA to pyridine ratio ranging from 0 to 1 . Pyr was used instead of $4 \mathrm{VP}$ to prevent polymerization of the vinyl groups of 4VP. We believe that this is allowed because the vinylgroup does not interact with $\mathrm{SaA}$, it only introduces a steric effect by its relative possition to the amine group [22]. Furthermore the pKa's of 4VP and Pyr are comparable and the formation of an (ionic) bond can therfore be monitored with Pyr instead of 4VP. 
Table 1: Solvent composition tested for complex formation of SaA with Pyr.

\begin{tabular}{lcll}
\hline Solvent & $\begin{array}{c}\text { MeOH content } \\
\text { in } \mathrm{H}_{2} \mathrm{O}[\mathrm{mol} \%]\end{array}$ & pH & Buffer \\
\hline $\mathrm{MeOH}$ & 100 & 6.0 & - \\
$\mathrm{MeOH} / \mathrm{H}_{2} \mathrm{O}$ & 30 & 2.5 & $10 \mathrm{mM}$ phosphate \\
$\mathrm{H}_{2} \mathrm{O}$ & 0 & 2.5 & $10 \mathrm{mM}$ phosphate \\
$\mathrm{MeOH} / \mathrm{H}_{2} \mathrm{O}$ & 30 & 4.1 & $10 \mathrm{mM}$ acetic acid/sodium acetate \\
\hline
\end{tabular}

All solutions were poured in a $1 \mathrm{~cm}$ quarts cuvette (Varian) 5 minutes after mixing and the UV-Vis spectra $(600-200 \mathrm{~nm})$ of the solutions were recorded with a UV-Vis spectrometer. One quarts cuvette with cap was used for all spectra to minimize deviations in the measurements caused by the differences in the cuvette and evaporation of the solvent. The data were treated as described below to form a Jobs plot.

Determination of the complex composition using UV-Vis spectroscopy with the continuous variation method

Formation of a complex between two molecules changes the excitation energy of electrons in the complex compared to the individual unbound molecules. The absorbence of a molecule, at a wavelength $\lambda\left(A_{\lambda}[-]\right)$ follows Lambert Beers law (Equation 1):

$\mathrm{A}_{\lambda}=\mathrm{c} \cdot \varepsilon_{\lambda} \cdot 1$

With $\mathrm{c}[\mathrm{mol} / \mathrm{l}]$ the concentration, $\varepsilon_{\lambda}\left[\mid \mathrm{mol}^{-1} \mathrm{~cm}^{-1}\right]$ the extinction coefficient at wavelength $\lambda$ and I [cm] the path length of the light through the sample. When SaA and Pyr are mixed and show no interaction with each other, the absorbance at a certain wavelength $\left(A_{\text {sum, }, \lambda}\right)$ is the sum of the absorbances of the mixture of the two individual molecules at a certain wavelength. Deviations from ideality are observed when the molecules show interaction(s) with each other. The excitation energies of the electrons change and in that case a different spectrum is visible compared to the spectrum of the unbound molecules. The sum of the absorbences of the pure components $\left(A_{\text {sum }, \lambda}\right)$ 
is no longer equal to the actual absorbence observed for the mixture $\left(A_{o b s, \lambda}\right)$ of the components. The difference $\left(\Delta A_{\lambda}\right)$ between $A_{o b s}, \lambda$ and $A_{\text {sum }}, \lambda$ is used to form a Job's plot (Equation 2).

$\Delta \mathrm{A}_{\lambda}=\mathrm{A}_{\mathrm{sum}, \lambda}-\mathrm{A}_{\mathrm{obs}, \lambda}=\left(\mathrm{c}_{\mathrm{SaA}} \cdot \varepsilon_{\mathrm{SaA}, \lambda}+\mathrm{C}_{\mathrm{pyr}} \cdot \varepsilon_{\mathrm{pyr}, \lambda}\right) \cdot \mathrm{I}-\mathrm{A}_{\mathrm{obs}, \lambda}$

With $\varepsilon_{\mathrm{SaA}, \lambda}$ and $\varepsilon_{\mathrm{pyr}, \lambda}$, the extinction coefficients of SaA and Pyr respectively, determined in separate experiments to calculate $A_{\text {sum }, \lambda} \cdot \Delta A_{\lambda}$ is determined for different ratios of functional monomer to target molecule, varying from 0 to $1 . \Delta \mathrm{A}_{\lambda}$ is then calculated and plotted as function of the ratio of molecule SaA to Pyr (Jobs plot). The maximum $\Delta \mathrm{A}_{\lambda}$ is observed at the highest concentration of complexed molecules and therefore indicates the stoichiometric ratio of SaA and Pyr in the complex.

\subsubsection{Membrane preparation}

UV polymerization as described by Schneider et al [11] was used for surface modification of the polypropylene membranes with an imprinted or non imprinted layer. The photoinitiator BEE was adsorbed on the internal and external surface of circular PP membranes $(d=25 \mathrm{~mm})$ by placing the membranes in a $3 \mathrm{ml}$ solution containing $100 \mathrm{mM}$ BEE dissolved in acetone. After ten minutes the membranes were removed from the initiator solution and dried in air without light. The functional layer on the membranes was prepared by placing the BEE coated membranes in $3 \mathrm{ml}$ reaction mixture solution in a petridish $(\mathrm{d}=30 \mathrm{~mm})$. The reaction mixture contained functional monomer 4VP, crosslinker DEGDM and $2.5 \mathrm{mM}$ initiator BEE as well. BEE was added to the solution to shift the adsorption equilibrium of the initiator towards adsorption on the membrane surface. Molecularly imprinted membranes (Table 2, MIM 1 and MIM5) were prepared by adding SaA to the reaction mixture. The stoichiometry of the complex (1.5 $4 \mathrm{VP}$ to $1 \mathrm{SaA})$ was determined with the continuous variation method (see 3.1) and was used as an indication for the composition of the imprinting reaction mixture. 4VP contains an inhibitor to prevent polymerization. The mixture was compensated for the presence of the inhibitor by increasing the ratio $4 \mathrm{VP}$ to SaA to 2 to 1 . The reactant concentration, reaction medium composition and $\mathrm{pH}$ were varied to investigate the reaction rates of the different solution systems. These variables influence the reaction rate and therefore the degree of coating of the membranes. Comparison of the membrane selectivity and 
capacity is only possible when membranes with the similar degree of coating are used and for that a degree of coating of $400 \mu \mathrm{g} / \mathrm{cm}^{2}$ was considered.

The composition for the different membrane series is shown in Table 2. The $\mathrm{pH}$ of the reaction mixture of NIM 4, NIM5, MIM5 was adjusted to $\mathrm{pH} 4.1$ by adding acetic acid. To cure the system, the mixture with the membranes was placed under a $365 \mathrm{~nm}$ UV light with a light intensity of 7.5 $\mathrm{mW} / \mathrm{cm}^{2}$ under nitrogen atmosphere. The degree of coating of the membranes depends on the UV cure time. The relation between the degree of coating and the UV cure time was determined for the different series. 
Table 2: Components of the reaction mixture for the preparation of membranes.

\begin{tabular}{|c|c|c|c|c|c|c|c|}
\hline $\begin{array}{l}\text { Membrane } \\
\text { series }\end{array}$ & Solvent & $\begin{array}{c}\text { MeOH content } \\
\text { [mol \%] }\end{array}$ & $\begin{array}{l}\text { pH } \\
{[-]}\end{array}$ & $\begin{array}{l}4 \mathrm{VP} \\
{[\mathrm{mM}]}\end{array}$ & $\begin{array}{c}\text { DEGDM } \\
\text { [mM] }\end{array}$ & $\begin{array}{l}\mathrm{SaA} \\
{[\mathrm{mM}]}\end{array}$ & $\begin{array}{l}\text { UV cure } \\
\text { time [min] }\end{array}$ \\
\hline NIM1 & $\mathrm{MeOH}$ & 100 & 6.0 & 20 & 300 & - & $5-30$ \\
\hline MIM1 & $\mathrm{MeOH}$ & 100 & 6.0 & 20 & 300 & 10 & 25 \\
\hline NIM2 & $\mathrm{MeOH}$ & 100 & 6.0 & 2 & 30 & - & $5-30$ \\
\hline NIM3 & $\mathrm{MeOH} / \mathrm{H}_{2} \mathrm{O}$ & 30 & 6.0 & 2 & 30 & - & $0.5-10$ \\
\hline NIM4 & $\mathrm{MeOH} / \mathrm{H}_{2} \mathrm{O}$ & 30 & 4.1 & 20 & 300 & - & $0.5-2$ \\
\hline \multirow[t]{2}{*}{ NIM5 } & $\mathrm{MeOH} / \mathrm{H}_{2} \mathrm{O}$ & 30 & 4.1 & 2 & 30 & - & $0.5-10$ \\
\hline & $\mathrm{MeOH}$ & & & & & & \\
\hline \multirow[t]{2}{*}{ MIM5 } & & 30 & 4.1 & 2 & 30 & 1 & 2.5 \\
\hline & $/ \mathrm{H}_{2} \mathrm{O}$ & & & & & & \\
\hline
\end{tabular}

After UV curing the membranes were immediately rinsed with ethanol. The unreacted components and template (only present in MIM) present in the membranes were removed by soxhlet extraction with ethanol for 24 to 48 hours. The effectiveness of the soxhlet extraction was examined by recording a UV spectrum of the permeate after filtration of double distilled water through the membranes. Washing of the membranes was completed when the adsorption at $300 \mathrm{~nm}$ in the recorded UV spectrum was no longer detectable. The degree of coating $\left[\mu \mathrm{g} / \mathrm{cm}^{2}\right]$ was determined gravimetrically according to the following equation (Equation 3 ) and determined as the average of 3 membranes.

Degree of coating $=\frac{m_{1}-m_{0}}{A_{m}}$ 
With $m_{0}[\mu \mathrm{g}]$ and $m_{1}[\mu \mathrm{g}]$ the mass of the membrane before and after coating respectively and $A_{m}$ $\left[\mathrm{cm}^{2}\right]$ the surface area of the membrane.

\subsubsection{Solid phase extraction}

$\mathrm{pH}$ dependence of $\mathrm{SaA}$ adsorption

The $\mathrm{pH}$ dependence of SaA adsorption on 4VP coated membranes was examined by performing extraction experiments. For these experiments, $2 \mathrm{ml}$ of $110^{-5} \mathrm{M} \mathrm{SaA}$ solution was filtered through the membranes under investigation at different $\mathrm{pH}$ in dead end mode. The membranes were prepared in methanol (NIM 1) with a degree of coating of $400 \mu \mathrm{g} / \mathrm{cm}^{2}$. The adsorption experiments were only performed with NIM 1 because it is assumed that all membranes have the same composition of the active layer. Consequently, the results for NIM 1 give a good indication of the $\mathrm{pH}$ adsorption dependence of the other membranes as well. The $\mathrm{pH}$ of the solution was adjusted from $\mathrm{pH} 2.5$ to $\mathrm{pH} 5$ by a $5 \mathrm{mM}$ buffer of SPM and phosphoric acid $(\mathrm{pH} 2.5,3.0,3.5)$ and SPM and SPD ( $\mathrm{pH}$ 5). The membranes were placed in a PFA syringe filter holder (Advantec, $25 \mathrm{~mm}$ ) that was adapted at the feed side with a valve for air removal. The solution was pumped through the membrane with a syringe pump at $1 \mathrm{ml} / \mathrm{min}$. The concentration of the solute in the permeate was determined by UV spectroscopy at $300 \mathrm{~nm}$.

The adsorption (A [\%]) on the membrane was determined from a mass balance over the membrane (Equation 4) and the obtained values are the average of 3 membranes.

SaA adsorption $=\frac{\left(c_{f}-c_{p}\right)}{c_{f}} 100 \%$

With $c_{f}[\mathrm{~mol} / /]$ and $c_{p}[\mathrm{~mol} / /]$ the concentration of solute in the feed and permeate respectively.

\section{Breakthrough experiments}

The SaA adsorption capacity of the imprinted and not imprinted membranes at high throughput volume was determined with a breakthrough experiment. The membranes were prepared in 
$\mathrm{MeOH} /$ water at a pH of 4.1 (NIM5 and MIM5) with a degree of coating of $400 \mu \mathrm{g} / \mathrm{cm}^{2}$. NIM 5 and MIM 5 were used since this series shows molecular imprinting as will be discussed in later. A solution of $110^{-5} \mathrm{M} \mathrm{SaA}($ at $\mathrm{pH} 2.5$ ) was filtered through the membrane at a flow rate of $1 \mathrm{ml} / \mathrm{min}$ as described in 3.4.1. The permeate was fractionated to $1 \mathrm{ml}$ volume fractions and the SaA concentration in these fractions was determined with an UV-Vis spectrometer.

\section{SaA adsorption as function of the degree of coating}

The effect of the degree of coating on the adsorption of SaA was investigated by filtration of $1 \cdot 10^{-5}$ $\mathrm{M} \mathrm{SaA}(\mathrm{pH} 2.5)$ through the membranes as described in 3.4.1. The membranes were prepared in methanol (NIM1) with varying degree of coating. All membranes (series 1 to 5 ) are assumed to have the same amount of crosslinker and functional monomer when the degree of coating is comparable.

\section{MIM selectivity}

The adsorption selectivity of the membranes was determined by performing extraction experiments by filtrating $2 \mathrm{ml}$ of $110^{-5} \mathrm{M} \mathrm{SaA}, 3 \mathrm{M}-\mathrm{SaA}$, LA or HBA solution through the membranes (Figure 4). The $\mathrm{pH}$ of the solution was adjusted to 2.5 using a $5 \mathrm{mM}$ buffer of SPM and phosphoric acid. The membranes were prepared in methanol (NIM1 and MIM1) and in methanol/water at a $\mathrm{pH}$ of 4.1 (NIM5 and MIM5) resulting in a degree of coating of $400 \mu \mathrm{g} / \mathrm{cm}^{2}$. The extraction experiments were performed as described above. The concentration solute in the permeate was determined by UV spectroscopy at $300 \mathrm{~nm}$ (SaA, 3M-SA, HBA) and HPLC (LA) as described below. The adsorption was determined from a mass balance over the membrane according to Equation 4 and the final values are an average of 3 membranes. 
<smiles>O=C(O)c1ccccc1O</smiles>

$\mathrm{SaA}$<smiles>Cc1cccc(C(=O)O)c1O</smiles>

$3 \mathrm{M}$ SaA<smiles>O=C(O)c1ccc(O)cc1</smiles>

4HBA<smiles>CC(O)C(=O)O</smiles>

LA

Figure 4: Molecular structures of SaA, 3M-SaA, 4HBA and LA.

The LA concentration was determined with a Waters HPLC with a 515 pump, a 2487 dual $\lambda$ adsorbance detector and Millenium ${ }^{32}$ (version 3.20) software. Analysis was carried out using a Resex ROA-organic acid $300 \times 7.8 \mathrm{~mm}$ column (Phenomenex). The mobile phase was $0.005 \mathrm{M}$ $\mathrm{H}_{2} \mathrm{SO}_{4}$. The column was operated at $90^{\circ} \mathrm{C}$ at a mobile phase flow rate of $0.3 \mathrm{ml} / \mathrm{min}$. Peak detection was performed with UV at a wavelength of $210 \mathrm{~nm}$.

\subsection{Results and discussion}

\subsubsection{Jobs plot}

Titration of SaA and Pyr in different solvents and solvent compositions was performed to evaluate the existence of complex at those conditions and to determine the stoichiometry of that complex. If a complex is observed, this is visible as a deviation of the observed absorbence, $\Delta \mathrm{A}_{\mathrm{obs}}$ from the ideal absorbence [23-27]. This deviation was only observed when $\mathrm{MeOH} /$ water at a $\mathrm{pH}$ of 4.1 was used as solvent. The corresponding Jobs plot at $230 \mathrm{~nm}$ is shown in Figure 5.

When other solvents are used, $A_{\text {sum }, \lambda}$ is equal to $A_{o b s, \lambda}$ indicating that complex formation between the molecules does not occur. These results are summarized in Table 3. Titrations in methanol at $\mathrm{pH} 6.0$ were performed as reference as under these conditions, complex formation is not expected based on the calculations of the pKa. 


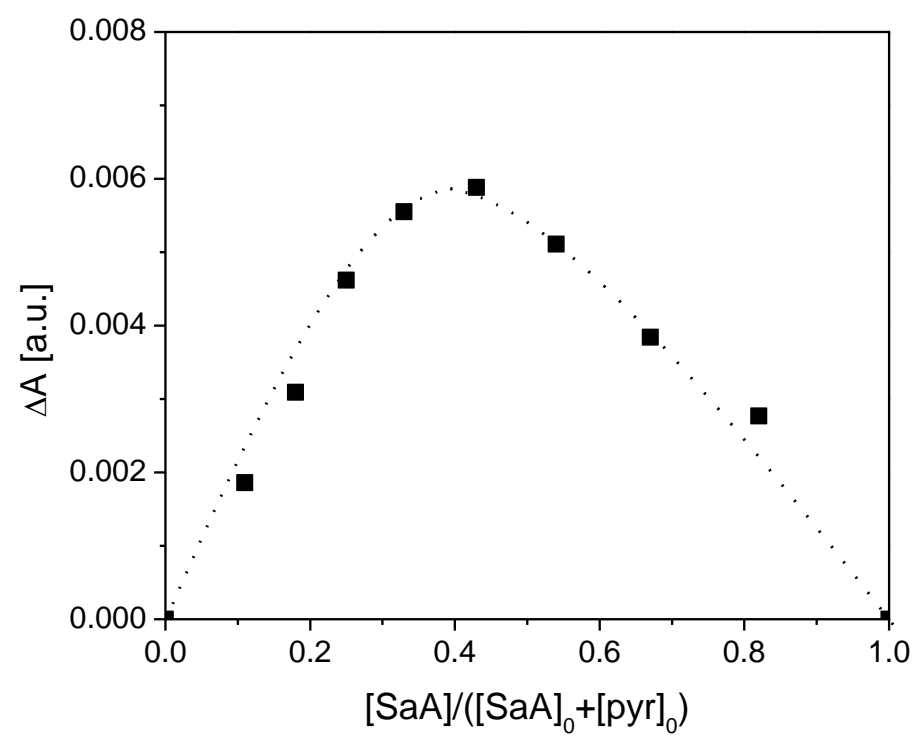

Figure 5: Jobs plot of SaA and Pyr in water at $\mathrm{pH} 4.1$.

Table 3: Complex formation of SaA and 4VP in different solvent compositions

\begin{tabular}{lccccc}
\hline Solvent & MeOH content & pH & Charge & Charge & Complex formation \\
& in $\mathrm{H}_{2}$ [ [mol\%] & & SaA & Pyr & Jobs plot \\
\hline $\mathrm{MeOH}$ & 100 & 6.0 & - & Neutral & No \\
$\mathrm{MeOH} / \mathrm{H}_{2} \mathrm{O}$ & 30 & 2.5 & Neutral & + & No \\
$\mathrm{H}$ & 0 & 2.5 & Neutral & + & No \\
$\mathrm{MeOH} / \mathrm{H}_{2} \mathrm{O}$ & 30 & 4.1 & - & + & Yes \\
\hline
\end{tabular}

The formation of a complex between $\mathrm{SaA}$ and $\mathrm{Pyr}$ in $\mathrm{MeOH} /$ water at $\mathrm{pH} 4.1$ was expected based on the calculations on the pKa of SaA and 4VP as presented in Figure 3. At those conditions both SaA and Pyr are charged and the molecules are able to form a complex. This result indicates that the complex formed between SaA and Pyr is ionic. Although no complex was formed at $\mathrm{pH} 2.5$, complex formation at $\mathrm{pH} 2.5$ was expected because at those conditions Pyr is protonated and charged while SaA is uncharged. Consequently, the negative $\pi$ orbital (dipole) of the aromatic ring should be able to form a bond with the positively charged Pyr. Such adsorption was indeed 
observed by Michailof et al. [28]. We cannot explain this discrepancy. It is possible that the shift of $\pi$ orbitals of SaA under the influence of the charged pyridine is too small to be observed by our experimental method. Other reasons can be that the equilibrium is not shifted towards the complex or that the buffer in this experiment interferes with the complex formation or the observation of the shift.

Figure 5 shows a distinct maximum $\Delta \mathrm{A}$, and consequently complex formation when the ratio $\mathrm{SaA}$ to Pyr is 1 to 1.5 at a relative SaA concentration of $\sim 0.4$. Unfortunately the method only provides quantitative information, it does not give information about the actual size, form or shape of the complex. There are many possibilities for complex formation, and although it is speculated that the acid-base binding is dominant, it is also possible that hydrogen bonding and $\pi-\pi$ stacking play a role, resulting in the formation of a Pyr-SaA-Pyr-SaA-Pyr sandwich. Computational chemistry could provide more information on this, but is beyond the scope of this work.

\subsubsection{Membrane preparation and characterization}

Degree of coating as function of the UV cure time

The reaction medium and reactant concentration have a strong influence on the polymerization kinetics and consequently the final coating layer, which is visualized by the degree of coating. The effect of medium, $\mathrm{pH}$, concentration and UV cure time (Table 2) on the degree of coating was investigated and the results are given in Figure 6. 


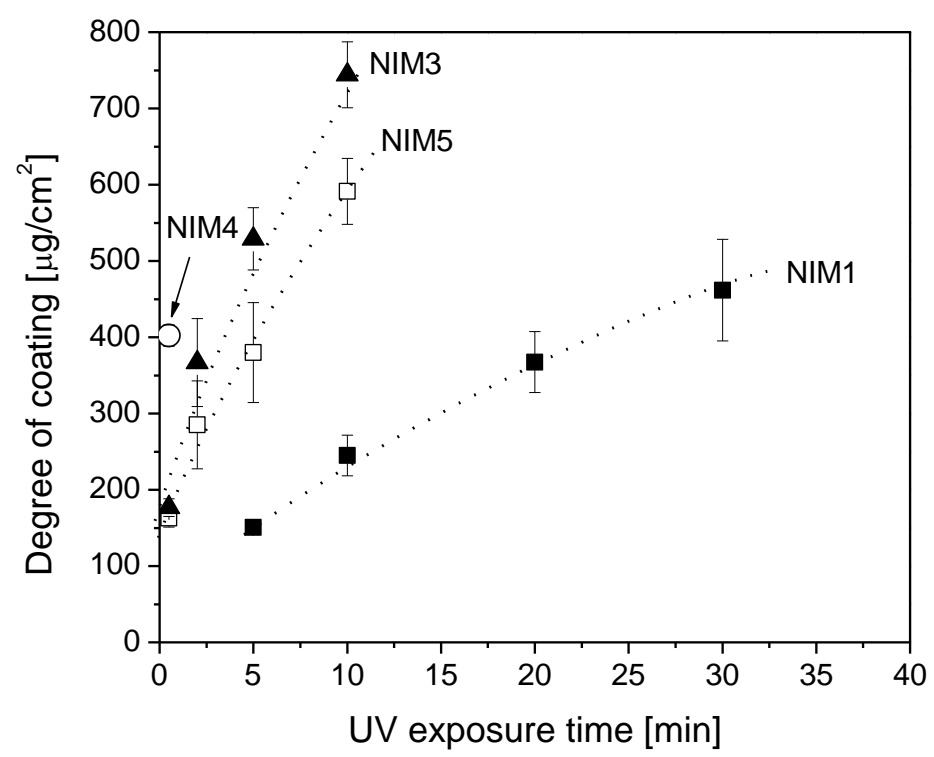

Figure 6: Degree of coating $\left[\mu \mathrm{g} / \mathrm{cm}^{2}\right]$ as function of the UV cure time $[\mathrm{min}]$ in methanol (- NIM1) and $40 \mathrm{~mol} \%$ methanol in water ( $\Delta$ NIM 3; ○ NIM 4; 口 NIM5). NIM 1, NIM2, NIM 3, at pH = 6; NIM4, NIM5 at pH 4.1.

Figure 6 shows the degree of coating as a function of the cure time for the different NIMs. The degree of coating of the NIM2 series (methanol, low concentration) was too low to show a consistent pattern and the data are not presented. UV curing of NIM4 at times longer than 0.5 minutes lead to a completely gelled reaction mixture. Consequently also these data are not included.

Figure 6 clearly shows the strong dependency on the reaction rate, expressed as the degree of coating, as function of the UV exposure time and the reaction medium. For all media, the degree of coating of the membranes increases with increasing UV exposure time, as was also shown by Schneider et al. [11]. The concentration of the reactants in $\mathrm{MeOH} /$ water (NIM3 and NIM5) is ten times lower than that in pure methanol (NIM1), while the degree of coating as function of the UV exposure time is lower for NIM1. When the concentrations are equal (NIM1 and NIM4) a cure time of 0.5 minutes in $\mathrm{MeOH} /$ water shows the same degree of coating as 30 minutes of crosslinking in pure methanol. These results show that the presence of water increases the reaction rate of the system. The degree of coating also varies when the $\mathrm{pH}$ is altered. At lower $\mathrm{pH}$, the reaction kinetics 
are slower, since the degree of coating as function of UV cure time is also lower when in that case (compare NIM5 and NIM 3). There are several possible explanations for the change in reaction rate when water is added or when the $\mathrm{pH}$ is altered. UV curing is a radical chain polymerization consisting of initiation, propagation and termination. Each of these steps can be affected by the introduction of other species (e.g. buffer, solvent), for instance by enhancing initiation, reducing radical chain transfer, reducing termination or even by concentration of the reactants in small solvent domains. It is beyond the scope of this research to investigate the specific reasons for the difference in reaction rates, but the results clearly show that the reaction rate and therefore the degree of coating varies when the system is altered. For a fair comparison of the different MIMs and NIMs, the reaction times are therefore varied to obtain membranes with comparable degrees of coating $\left(400 \mu \mathrm{g} / \mathrm{cm}^{2}\right)$.

\section{Effect of $\mathrm{pH}$ and $\mathrm{pKa}$}

The pKa of a functional group is known to deviate from the ideal pKa when it is embedded in a strongly crosslinked matrix, resulting in an apparent pKa [29, 30]. This effect has a strong influence on the $\mathrm{pH}$ range in which $\mathrm{SaA}$ adsorption is possible, since 4VP and SaA need to be protonated and deprotonated respectively for SaA adsorption to occur. The SaA adsorption on the membranes as function of the $\mathrm{pH}$ is investigated and presented in Figure 7 for NIMs prepared in methanol (NIM1) with a degree of coating of $400 \mu \mathrm{g} / \mathrm{cm}^{2}$. 


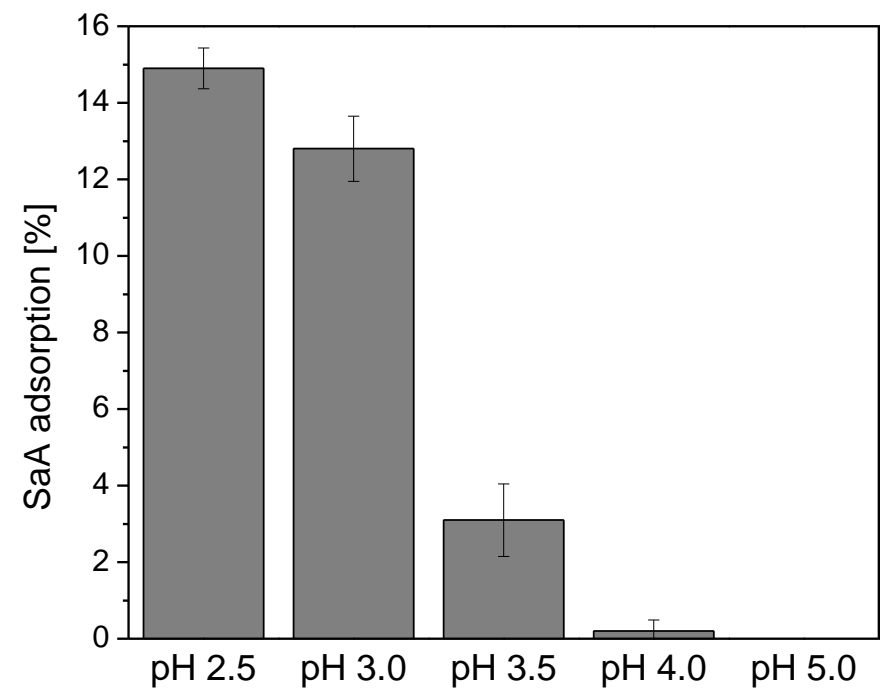

Figure 7: SaA adsorption (relative to feed concentration $1 \cdot 10^{-5} \mathrm{M}$ ) in NIM1 as function of $\mathrm{pH}$.

Figure 7 shows that at $\mathrm{pH} 2.5$, the adsorption of $\mathrm{SaA}$ in the membranes is almost $15 \%$ of the $\mathrm{SaA}$ concentration in the feed. SaA adsorption strongly decreases when the $\mathrm{pH}$ increases. At $\mathrm{pH} 5$, adsorption is not observed. These results seem to be in contrast with the results obtained from the continuous variation method. In that case, complex formation was not visible at $\mathrm{pH} 2.5$, but was observed at $\mathrm{pH}$ 4.1. When ionic interactions are presumed dominant, it is expected that the free uncrosslinked molecules would show strong interactions in the range between the pKa of SaA (pKa 2.9) and that of the base (pKa 5.4), as was also observed by the continuous variation method. The deviation from this adsorption behavior is believed to be caused by a local $\mathrm{pH}$ shift occurring in the membrane. This phenomenon was previously observed in ion exchange membranes [29] and MIMs [11] and assumed to be caused by the high degree of crosslinking of 4VP. Higher degrees of crosslinking of the material lower the water content in the material. This reduces the proton conductivity as proton transport is governed by a hopping mechanism from water molecule to water molecule. Consequently, a higher proton concentration in the solution (lower $\mathrm{pH}$ ) is required to reach the same degree of protonation of $4 \mathrm{VP}$ compared to when 4VP is not crosslinked. Because of their thickness, MIPs do not show clear pKa transitions [11], which makes it hard to study this effect on the adsorption properties. The transition is less clear because the ionic groups are less 
accessible and penetration through the thick layer is slow, resulting is a poorly defined pKa curve. Due to there relatively low thickness, MIMs on the other hand, have ionic groups that are much better accessible and they show a clearer pKa transition. Therefore, MIMs are better candidates to study the adsorption conditions necessary for good recognition properties of imprints. Molecular imprinting shows many examples of increased adsorption or retention (when MIPs are applied in HPLC columns) of phenolic acids [2, 32, 35] when the $\mathrm{pH}$ is lowered. Michailof et al. [28] imprinted caffeic acid and p-hydroxybenzoic acid for solid phase extraction in aqueous environments and also found that the binding $\mathrm{pH}$ is essential for the recognition of the acids. They showed that lowering the $\mathrm{pH}$ increased the adsorption of the acids due to acid base interactions. These acid base interactions were not present during the imprinting in the organic solvent and thus interfered with the recognition properties. All other research on SaA imprinting showed no or very low adsorption or retention when the solution was not buffered [18] or buffered at higher $\mathrm{pH}$ [4], as SaA adsorption is only possible at lower $\mathrm{pH}$.

These results show that the applicable $\mathrm{pH}$ range for MIPs and MIMs may vary from the expected $\mathrm{pH}$ (based on PKa) due to the strong degree of crosslinking of the functional groups. In molecular imprinting this effect should be considered when MIPs and MIMs are used, since this may change the type of bonds that is available for molecular recognition.

\section{Breakthrough experiments}

Breakthrough experiments were performed to determine the capacity for SaA adsorption of the membranes (NIM5 and MIM5). The results are given in Figure 8. 


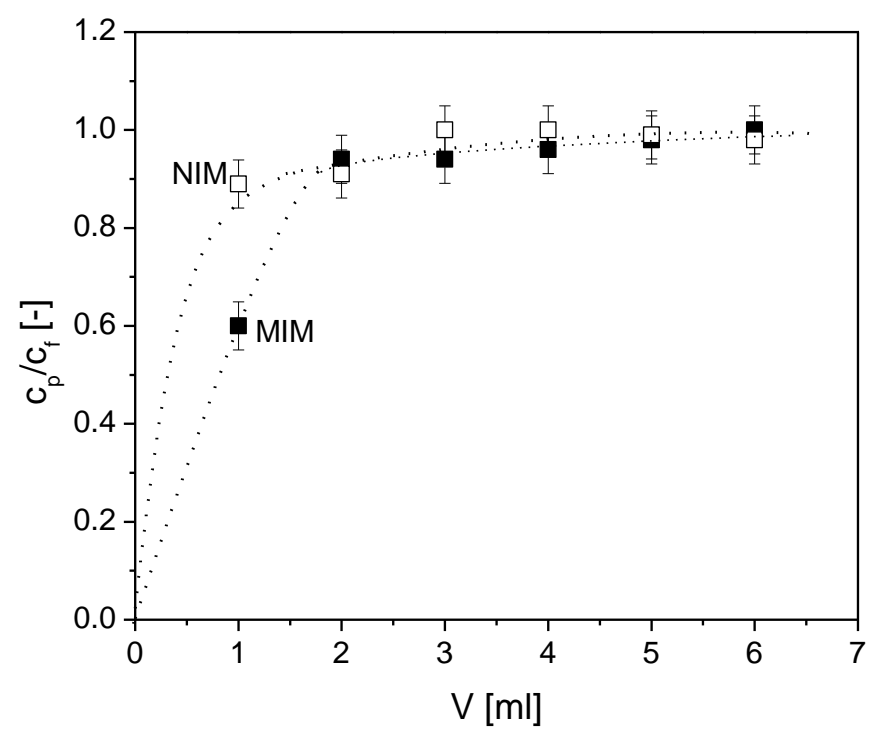

Figure 8: SaA breakthrough as function of filtered volume SaA solution $\left(1 \cdot 10^{-5} \mathrm{M}\right):$ - MIM5; $\square$ NIM5 at pH 2.5 .

Figure 8 shows that when $1 \mathrm{ml}$ of SaA solution $\left(1 \cdot 10^{-5} \mathrm{M}\right)$ is filtered through NIM5 and MIM5, SaA is immediately present in the permeate. The concentration of the permeate of NIM5 is $90 \%$ of that of the feed while the concentration of the permeate from MIM5 is $60 \%$ of that of the feed. Filtration of $2 \mathrm{ml}$ or more, results in equal SaA permeate concentrations for both NIM5 and MIM5.

Already after filtration of $2 \mathrm{ml}$, the membrane system seems to be close to saturation and it seems fully saturated after filtration of $6 \mathrm{ml}$. It is possible that more adsorption sites are available but that these are only accessible by very slow diffusion through the cavities of the imprinted sites. Assuming that there is still some additional capacity available, using that would require very long equilibrium times, which makes that anyway non attractive for practical purposes.

Due to the limited data, no attempt has been made to determine the adsorption kinetics from this graph, but it is reasonable to assume that the adsorption kinetics are fast since adsorption is observed while the flow rates are very high. The total bed volume (BV) of the membrane is $0.7 \mathrm{ml}$. At a flowrate of $1 \mathrm{ml} / \mathrm{min}$, this results in a filtration rate as high as $788 \mathrm{BV} / \mathrm{h}$. For comparison, due to adsorption kinetic limitations and high pressure drops, conventional adsorbers typically operate at flow rates of 1 to $40 \mathrm{BV} / \mathrm{h}$ [31-33]. 
Due to their fast kinetics and low pressure drops membrane adsorbers can be used at much higher flow rates [11]. Research with adsorption of SaA and other phenolic acids [4] on grinded imprinted particles shows that the adsorption capacity of the imprinted particles is reached after more than 10 hours. The membranes show very fast adsorption kinetics and reach the maximum adsorption capacity within 2 minutes only. This is due to the high surface area to volume ratio of the imprinted sites due to coating of the imprinted layer on the internal surface of the porous membrane. Consequently, adsorption sites are located at or near the surface of the membrane, allowing short diffusion lengths and times. Application of membranes in commercial adsorption applications will demand different membrane configurations [34] (e.g. spiral wound or hollow fiber membranes in series). The high throughput possible with these membranes is a big step towards a viable process with molecularly imprinted materials.

\section{SaA adsorption as function of degree of coating}

$\mathrm{SaA}$ adsorption as function of the degree of coating was investigated with the membranes NIM1 to study the effect of the support and the coating on SaA adsorption. As such the specific effect of the presence of imprints is not studied in that case. The unreacted monomers in the membrane were removed by soxhlet extraction with ethanol and the effectiveness of Soxhlet extraction was investigated by filtration of water through the membrane. Filtration of water through the membranes showed that membranes with a degree of coating lower than $400 \mu \mathrm{g} / \mathrm{cm}^{2}$ were free of unreacted monomers after 24 hours of soxhlet extraction. Membranes with a degree of coating between 400 and $800 \mu \mathrm{g} / \mathrm{cm}^{2}$ needed at least $48 \mathrm{~h}$ of soxhlet extraction before the membranes were clean. Membranes with a degree of coating higher than $1000 \mu \mathrm{g} / \mathrm{cm}^{2}$ kept leaching unreacted monomer from the coating even after 5 days of soxhlet extraction. SaA adsorption is therefore only performed with membranes with a degree of coating below $800 \mu \mathrm{g} / \mathrm{cm}^{2}$. While rinsing the membranes with water to see if desorption was complete, it was observed that wetting of the membranes with water required lower initial pressures when the degree of coating was higher. This is due to the hydrophilic nature of the crosslinker DEGDM. SaA adsorption as function of the degree of coating is performed by filtering $2 \mathrm{ml} 1110^{-5} \mathrm{M} \mathrm{SaA}$ through the membrane at $\mathrm{pH} 2.5$ (Figure 9) as at this $\mathrm{pH}$ $\mathrm{SaA}$ adsorption is most favorable (Figure 7). 


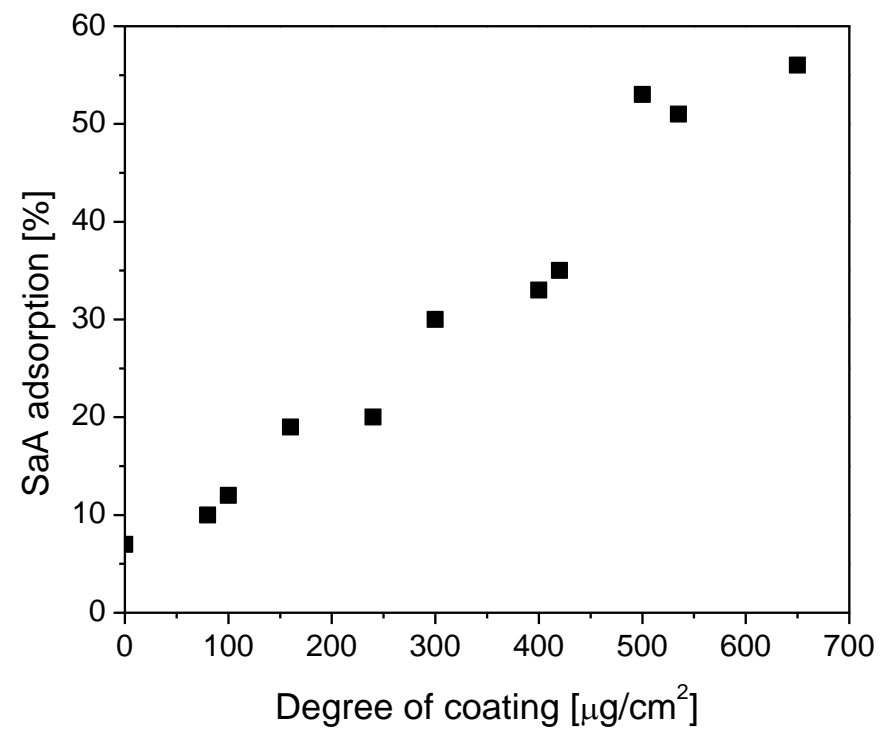

Figure 9: SaA adsorption (relative to feed concentration $110^{-5} \mathrm{M}$ ) as function of degree of coating on NIM 1 at pH 2.5.

Figure 9 shows that $\mathrm{SaA}$ adsorption is $7 \%$ when the membranes are not coated and adsorption increases linearly with increasing degree of coating. This linearity indicates that the adsorption kinetics are fast and not limiting at these flow rates. Although hydrophobic interactions between the hydrophobic ring of $\mathrm{SaA}$ and the hydrophobic backbone of the support were expected to play a role in SaA adsorption, the linear character indicates that the main SaA adsorption mechanism is ionic. By increasing the degree of coating, the hydrophilicity of the membrane increases as is indicated by the easiness of wetting of the membrane. However, the increased hydrophilicity does not reduce the SaA adsorption, instead adsorption is increased. This suggest that the main mechanism of adsorption is based on the much stronger ionic interactions, as was also observed with phenolic acid adsorption on pyridine at low pH by Michailof et al. [28]. This shows that when this system is used for MIMs, the PP support will not provide unspecific adsorption. Furthermore, when the membranes are only coated with $4 \mathrm{VP}, 4 \mathrm{VP}$ is accessible for SaA adsorption to a degree of coating of $800 \mu \mathrm{g} / \mathrm{cm}^{2}$. 


\section{MIM selectivity}

The presence of molecular imprints was verified by measuring the SaA adsorption on NIMs and MIMs (NIM1, MIM1, NIM5, MIM5). These results are shown in Figure 10.

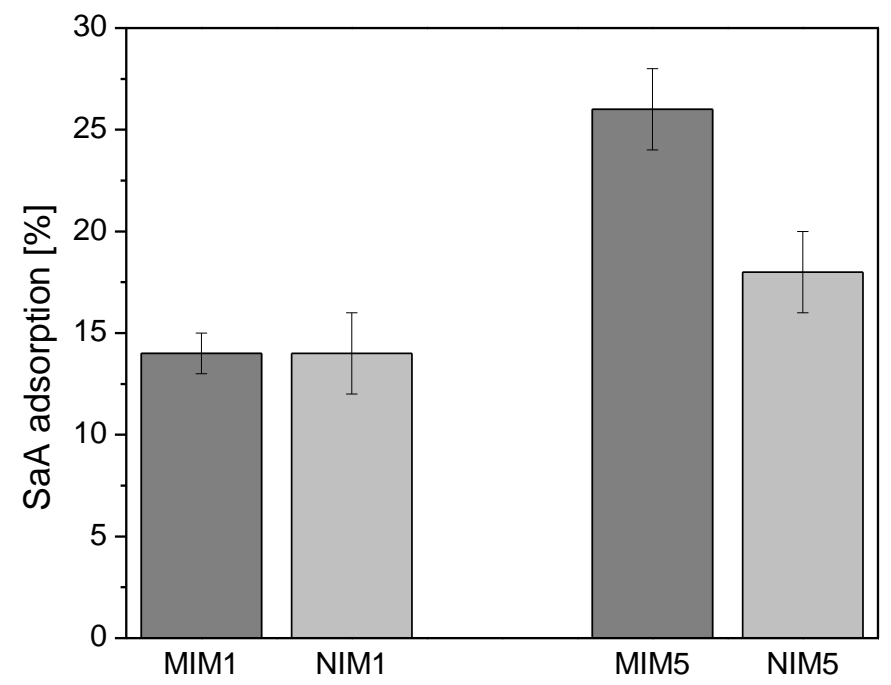

Figure 10: SaA adsorption (relative to feed concentration $110^{-5} \mathrm{M}$ ) on NIM and MIM polymerized in methanol (MIM1 and NIM1) and MeOH/water prepared at $\mathrm{pH} 4.1$ (MIM5 and NIM5) at pH 2.5.

The SaA adsorption at the NIM and MIM prepared in methanol is $14 \%$ for both membranes. The adsorption of the membranes prepared in $\mathrm{MeOH} /$ water is, for both the NIM and the MIM, higher. But more important, with adsorptions of $18 \%$ and $26 \%$ for NIM5 and MIM5, respectively, the results proof the presence of selective adsorption sides in this case. The similar SaA adsorption in NIM and MIM prepared in methanol was expected based on the results obtained with the continuous variation method. It indicates that the membranes prepared at this condition do not contain an imprint, or the imprint is not visible at these conditions. The adsorption that is observed is non specific adsorption of SaA on 4VP groups present in the membrane. This also indicates that the presence of $\mathrm{SaA}$ in the reaction mixture does not influence the number of $4 \mathrm{VP}$ groups present in the membrane. Adsorption of $\mathrm{SaA}$ on the membranes prepared in $\mathrm{MeOH} /$ water do show a difference in SaA adsorption between NIM and MIM. The MIM membranes show significantly 
higher SaA adsorption than the NIM membranes. This can be attributed to the specific adsorption of the molecular imprints.

SaA adsorption is slightly higher in the NIM prepared in $\mathrm{MeOH} /$ water than in the NIM prepared in methanol. A possible explanation for this higher adsorption could be a higher concentration of 4VP present in the membranes. It is possible that polymerization conditions with water favor 4VP polymerization over DEGD polymerization a little more than in methanol alone.

Finally, the SaA adsorption selectivity of the MIMs compared to the NIMs prepared in $\mathrm{MeOH} /$ water was investigated by filtering $\mathrm{SaA}, 3 \mathrm{M}-\mathrm{SaA}, \mathrm{HBA}$ or LA through the membranes (each component individually). NIM5/MIM5 was chosen, because of the presence of imprints in MIM5. The results are shown in Figure 11.

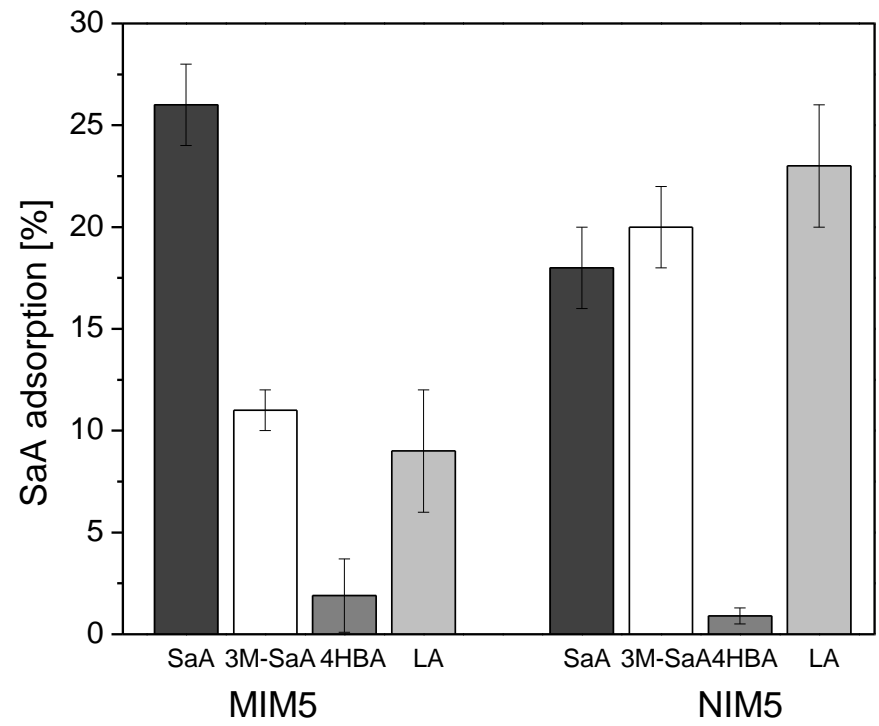

Figure 11: Adsorption of SaA, 3M-SaA, LA and 4HBA (relative to feed concentration $110^{-5} \mathrm{M}$ ) on MIM5 and NIM5 at pH 2.5 .

Figure 11 shows the selectivity of MIM5 for SaA adsorption compared to that of NIM5.. The SaA adsorption in MIM5 is $26 \%$, while $3 \mathrm{M}$-SaA, LA and 4HBA adsorption is clearly lower with $11 \%, 2 \%$ and $9 \%$ respectively. This proofs the selective adsorption of the imprints present in the membrane. In NIM5 on the other hand, SaA adsorption is even lower than in MIM5 (18\%), while 3M-SaA and 
LA are higher (20\% and $23 \%$ respectively). 4HBA adsorption is in both cases very low compared to the other acid adsorptions with $2 \%$ in MIM5 and 1\% in NIM5.

$\mathrm{SaA}$ (pKa 2.9) and 3M-SaA (pKa 2.8) have similar pKa's and the lower adsorption of 3M-SaA in MIM5 can be attributed to a hindered adsorption caused by the methyl group of 3M-SaA. This effect was also observed by Simon et al. [35]. They found that shape selectivity of other groups than the functional groups plays a major role in templates with 2 or less functional groups. In that case, the imprint selectivity is not only based on the ordening of functional groups.

The methyl group of 3M-SaA does not reduce its adsorption capacity on NIM5. This is unexpected since $3 \mathrm{M}-\mathrm{SaA}$ is bulkier and therefore its adsorption in the non-imprinted sites is expected to be lower compared to that of SaA. This is an indication that the unspecific (not imprinted) 4VP groups are not ordered around a cavity, and that shape and size are unimportant for this unspecific adsorption. 4HBA adsorption is low in both MIM5 and NIM5, which makes it difficult to assess the adsorption potential of 4HBA of the MIM compared to that of the NIM. The low adsorption of 4HBA is likely due to the higher pKa of 4 HBA (4.5), causing the molecule to be protonated and uncharged. 4HBA is able to form hydrogen bonds, however Figure 11 shows that this does not lead to a high adsorption. Although literature has shown that $\pi-\pi$ interactions are important when phenolic acids are imprinted with 4VP [28], the low adsorption of 4HBA indicates that it plays a minor role in this system. Most likely this is due to the lower strength of $\pi-\pi$ interactions compared to ionic interactions.

LA adsorption is lower in the MIM and higher in the NIM compared to SaA adsorption. LA also has a higher pKa than SaA (3.8) but in contrast to 4HBA, LA shows significant adsorption. The pKa of LA is higher than that of SaA and 3M-SaA but lower than that of $4 \mathrm{HBA}$ and it is possible that the molecule is still (partially) protonated and therefore shows higher adsorption than 4HBA. The higher LA than SaA adsorption in the NIM is expected because LA is a smaller molecule than SaA and adsorption sites can be more easily reached in that case. As such, it would be reasonable to assume that this would also be valid for the small cavities formed by the imprints of SaA. That this is not the case is caused by the different three dimensional ordening of the functional groups compared to the three dimensional ordening of the functional groups of SaA, as well as the different shape of the non specific groups of LA. This finding is in accordance with the work of Simon et al. [35]. They found that different ordening of specific groups introduces selectivity, as 
well as shape selectivity of nonfunctional groups, even if the nonfunctional groups are smaller than those of the template.

It is difficult to find appropriate molecules for selectivity studies of small molecules because an alteration of a small molecule affects the properties of the molecules (e.g. solubility, pKa, electrostatic interactions). However the adsorption tests of these different molecules clearly show that SaA has been imprinted in the membrane. These results also indicate that differences in shape (bulky group of $3 \mathrm{M}-\mathrm{SaA}$, different shape of LA) and ordening of functional groups (LA) can be used for preferential adsorption of molecules by molecular imprinting.

The results obtained from the pKa determination show that the pKa of the membrane changes during UV curing (see 4.1.2). The apparent pKa of 4VP is reduced from 5.62 to approximately 3.25 when $4 \mathrm{VP}$ is incorporated in the membrane. We realize that the $\mathrm{pH}$ inside the membrane (and in the imprint) changes during UV curing and that this probably also affects the complex between SaA and 4VP that is formed prior to UV curing. It is not clear how this affects the molecular imprinting, but the selectivity results show that an imprint is achieved. We believe that UV curing preserves the three dimensional ordening directly at the start of the UV curing when the polymer starts to gellate. As such, it captures the template, but at the same time, the crosslink density is not sufficiently high yet to alter the $\mathrm{pH}$. Further crosslinking then reduces the $\mathrm{pH}$ in the membrane, while the first loosely preserved three dimensional structure is tightened further. This crosslinking effect on the change of $\mathrm{pH}$ also clearly determines the applicable $\mathrm{pH}$ range for molecular recognition. Although less dominant and visible when more and other bonds (e.g. charge-dipole, dipole-dipole) are studied, this phenomenon will also affect other complex formations and should be considered.

We realize that unspecific acid adsorption on the membranes is observed. This research focuses on the optimal solvent conditions to enhance ionic interactions between an acidic template and a basic functional monomer, but there are many other aspects that could be investigated to further optimize the imprints of these membranes. Other commercial and non commercial functional monomers have been investigated to improve molecular imprinting of carboxylic acids [22, 36, 37] and altering the crosslinker, crosslinker length $[38,39]$ and concentration $[40]$ has also shown to 
improve molecular imprinting. This makes a rational design of MIPs sometimes difficult and a greater understanding is still necessary to make full use of the potential of molecular imprinting.

This research shows that molecular imprinting is a powerful tool to develop highly selective membrane adsorbers for biorefinery applications. Reaction medium and conditions are essential and allow tailoring required functionalities and selectivities. Molecular imprinting of SaA is possible at carefully chosen conditions such that ionic bonds are formed. Imprinting of acids by means of the ionic bond was achieved at low $\mathrm{pH}$ in a solvent that mimics the aqueous environment.

\title{
7.6 Conclusions
}

In this work, we show the successful preparation of molecularly imprinted membranes for the selective adsorption of acids. The effect of the solvent has been investigated and results show that careful selection of the solvent is required for successful molecular imprinting and recognition of SaA in aqueous conditions. We identified the optimal conditions of the solvent for complex formations by the continuous variation method. The optimal condition for SaA imprinting is at low $\mathrm{pH}$ in a solvent that mimics the aqueous environment. Complex formation between SaA and Pyr only occurs when both molecules are charged and ionic interactions are possible. Imprinting of SaA at these conditions leads to a membrane that selectively adsorbs $\mathrm{SaA}$ at low $\mathrm{pH}$ due to a local $\mathrm{pH}$ shift inside the membrane.

The membranes investigated show clear $\mathrm{pH}$ transitions and fast kinetics. Consequently, molecular imprinting of membranes (MIMs) is a very interesting technology for the selective recovery or separation of specific molecules from complex feed mixtures.

\subsection{Abbreviations and symbols}

\author{
Abbreviations
}

3M-SaA 3-methyl salicylic acid

4HBA 4-hydroxybenzoic acid 


$\begin{array}{ll}\text { 4VP } & \text { 4-Vinylpyridine } \\ \text { BEE } & \text { Benzoin ethyl ether } \\ \text { DEGDM } & \text { Diethyleneglycoldimethacrylate } \\ \text { LA } & \text { Lactic acid } \\ \text { MeOH } & \text { Methanol } \\ \text { MIM } & \text { Molecularly imprinted membrane } \\ \text { MIP } & \text { Molecularly imprinted polymer } \\ \text { NIM } & \text { Not imprinted membrane } \\ \text { NIP } & \text { Not imprinted polymer } \\ \text { Pyr } & \text { Pyridine } \\ \text { SaA } & \text { Salicylic acid } \\ \text { SPD } & \text { Sodium phosphate dibasic anhydrous } \\ \text { SPM } & \text { Sodium phosphate monobasic monohydrate }\end{array}$

Symbols

A Adsorption [\% of feed]

$\mathrm{A}_{\lambda} \quad$ Adsorbence at $\lambda[-]$

$A_{m} \quad$ Membrane surface area $\left[\mathrm{cm}^{2}\right]$

$A_{o b s, \lambda} \quad$ Observed absorbence of mixture pyr and SaA at $\lambda[-]$

$A_{\text {sum }, \lambda} \quad$ Sum of absorbence of pure Pyr and SaA at $\lambda[-]$

BV Bedvolume [ml]

C Concentration $[\mathrm{mol} / \mathrm{l}]$

$\mathrm{c}_{\mathrm{f}}, \mathrm{c}_{\mathrm{p}} \quad$ Feed and permeate concentraton [mol/l]

$\Delta \mathrm{A}_{\lambda} \quad$ Difference between $\mathrm{A}_{\mathrm{obs}, \mathrm{l}}$ and $\mathrm{A}_{\text {sum }, \lambda}[-]$

$\varepsilon_{\lambda} \quad$ Extincion coefficient at $\lambda\left[\mathrm{Imol}^{-1} \mathrm{~cm}^{-1}\right]$

I Pathlength of light $[\mathrm{cm}]$

$\lambda \quad$ Wavelength [nm]

$\mathrm{m}_{1}, \mathrm{~m}_{2} \quad$ Membrane mass $[\mu \mathrm{g}]$ before and after coating

$\mathrm{V}_{\mathrm{p}} \quad$ Volume permeate [ml] 


\subsection{Acknowledgments}

This work was carried out within project SC-00-04 of the Institute for Sustainable Process Technology (ISPT), The Netherlands.

\subsection{References}

1. Hirs, C.H.W., Moore, S., and Stein, W.H., The Chromatography of Amino Acids on Ion Exchange Resins. Use of Volatile Acids for Elution. Journal of the American Chemical Society, 1954. 76(23): p. 6063-6065.

2. Haupt, K., Dzgoev, A., and Mosbach, K., Assay System for the Herbicide 2,4Dichlorophenoxyacetic Acid Using a Molecularly Imprinted Polymer as an Artificial Recognition Element. Analytical Chemistry, 1998. 70(3): p. 628-631.

3. Weiss, R., et al., Molecular imprinting and solid phase extraction of flavonoid compounds. Bioseparation, 2001. 10(6): p. 379-387.

4. Byun, H.-S., et al., Selective separation of aspirin using molecularly imprinted polymers. Separation and Purification Technology, 2010. 74(1): p. 144-153.

5. Monier, M. and El-Sokkary, A.M.A., Preparation of molecularly imprinted cross-linked chitosan/glutaraldehyde resin for enantioselective separation of l-glutamic acid. International Journal of Biological Macromolecules. 47(2): p. 207-213.

6. Sueyoshi, Y., Fukushima, C., and Yoshikawa, M., Molecularly imprinted nanofiber membranes from cellulose acetate aimed for chiral separation. Journal of Membrane Science, 2010. 357(1-2): p. 90-97.

7. Ouyang, R., Lei, J., and Ju, H., Surface molecularly imprinted nanowire for protein specific recognition. Chemical Communications, 2008(44): p. 5761-5763.

8. Yan, M. and Ramström, O., Molecularly Imprinted Materials: Science and Technology. 2004: Taylor \& Francis.

9. Mayes, A.G. and Mosbach, K., Molecularly imprinted polymers: useful materials for analytical chemistry? Trends in Analytical Chemistry, 1997. 16(6): p. 321-332.

10. Ulbricht, M., Membrane separations using molecularly imprinted polymers. Journal of Chromatography B, 2004. 804(1): p. 113-125.

11. Schneider, F., et al., Comparison of thin-layer and bulk MIPs synthesized by photoinitiated in situ crosslinking polymerization from the same reaction mixtures. Journal of Applied Polymer Science, 2005. 98(1): p. 362-372.

12. Biorefenery. Available from: $h$ ttp://www.biorefinery.nl. [cited 2011 july 8]

13. Alexander, C., et al., Molecular imprinting science and technology: a survey of the literature for the years up to and including 2003. Journal of Molecular Recognition, 2006. 19(2): p. 106-180.

14. Donato, L., Figoli, A., and Drioli, E., Novel composite poly(4-viny/pyridine)/polypropylene membranes with recognition properties for (S)-naproxen. Journal of Pharmaceutical and Biomedical Analysis, 2005. 37(5): p. 1003-1008.

15. Kempe, M., Mosbach, K., and Fischer, L., Chiral separation using molecularly imprinted heteroaromatic polymers. Journal of Molecular Recognition, 1993. 6(1): p. 25-29.

16. Branch, G.E.K. and Yabroff, D.L., The Anomalous Strength of Salicylic Acid. Journal of the American Chemical Society, 1934. 56(12): p. 2568-2570.

17. Huang, X., et al., Molecular imprinting of nitrophenol and hydroxybenzoic acid isomers: effect of molecular structure and acidity on imprinting. Journal of Molecular Recognition, 2003. 16(6): p. 406-411.

18. Park, H.-R., et al., Separation of hydroxybenzoic acid isomers using the molecular imprinting technique. Journal of Applied Polymer Science, 2007. 105(5): p. 2824-2829.

19. Rived, F., et al., Acidity in methanol-water. Analytica Chimica Acta, 2001. 439(2): p. 315333. 
20. Subirats, X., Rosés, M., and Bosch, E., On the Effect of Organic Solvent Composition on the $\mathrm{pH}$ of Buffered HPLC Mobile Phases and the $\mathrm{pK}$ a of Analytes-A Review. Separation \& Purification Reviews, 2007. 36(3): p. 231-255.

21. Hirose, K., A Practical Guide for the Determination of Binding Constants. Journal of Inclusion Phenomena and Macrocyclic Chemistry, 2001. 39(3): p. 193-209.

22. Simon, R.L. and Spivak, D.A., Performance analysis of molecularly imprinted polymers for carboxylate and aminophosphate templates using commercially available basic functional monomers. Journal of Chromatography B-Analytical Technologies in the Biomedical and Life Sciences, 2004. 804(1): p. 203-209.

23. Chen, W.-Y., Chen, C.-S., and Lin, F.-Y., Molecular recognition in imprinted polymers: thermodynamic investigation of analyte binding using microcalorimetry. Journal of Chromatography A, 2001. 923(1-2): p. 1-6.

24. Jie, Z. and Xiwen, H., Study of the nature of recognition in molecularly imprinted polymer selective for 2-aminopyridine. Analytica Chimica Acta, 1999. 381(1): p. 85-91.

25. Qu, G., et al., Metal ion mediated synthesis of molecularly imprinted polymers targeting tetracyclines in aqueous samples. Journal of Chromatography B, 2009. 877(27): p. 31873193.

26. Tunc, Y., et al., Comonomer effects on binding performances and morphology of acrylatebased imprinted polymers. Polymer, 2006. 47(20): p. 6931-6940.

27. Wolman, F.J., et al., Peptide imprinted polymer synthesized by radiation-induced graft polymerization. Reactive and Functional Polymers, 2006. 66(11): p. 1199-1205.

28. Michailof, C., Manesiotis, P., and Panayiotou, C., Synthesis of caffeic acid and phydroxybenzoic acid molecularly imprinted polymers and their application for the selective extraction of polyphenols from olive mill waste waters. Journal of Chromatography A, 2008. 1182(1): p. 25-33.

29. Franck-Lacaze, L., Sistat, P., and Huguet, P., Determination of the pKa of poly (4vinylpyridine)-based weak anion exchange membranes for the investigation of the side proton leakage. Journal of Membrane Science, 2009. 326(2): p. 650-658.

30. Franck-Lacaze, L., et al., Protonation and diffusion phenomena in poly(4-vinylpyridine)based weak anion-exchange membranes. Journal of Membrane Science, 2009. 340(1-2): p. 257-265.

31. Dow, Product Information Dowex Optipore L493 and V493.

32. Lanxess, Product information Lewatit Monoplus M 500. 2010.

33. Lanxess, Product information Lewatit MP 62. 2010.

34. Borneman, Z., Particle Loaded Membrane Chromatography, in Ph.D. thesis. 2006, University of Twente: Enschede.

35. Simon, R., Collins, M.E., and Spivak, D.A., Shape selectivity versus functional group preorganization in molecularly imprinted polymers. Analytica Chimica Acta, 2007. 591(1): p. 716.

36. Lulinski, P., Dana, M., and Maciejewska, D., Synthesis and characterization of 3,4dihydroxyphenylacetic acid imprinted polymers. Polymer International. 61(4): p. 631-638.

37. Spivak, D. and Shea, K.J., Molecular imprinting of carboxylic acids employing novel functional macroporous polymers. Journal of Organic Chemistry, 1999. 64(13): p. 46274634.

38. Gong, C., Wong, K.-L., and Lam, M.H.W., Photoresponsive Molecularly Imprinted Hydrogels for the Photoregulated Release and Uptake of Pharmaceuticals in the Aqueous Media. Chemistry of Materials, 2008. 20(4): p. 1353-1358.

39. Byrne, M.E., Hilt, J.Z., and Peppas, N.A., Recognitive biomimetic networks with moiety imprinting for intelligent drug delivery. Journal of Biomedical Materials Research Part A, 2008. 84A(1): p. 137-147.

40. Park, H.R., et al., Molecular imprinting polymers for the separation of toluic acid isomers. Journal of Applied Polymer Science, 2005. 96(3): p. 650-654. 


\section{Discussion and Outlook}

\subsection{General Approach}

This thesis addresses several strategies to improve adsorption technology for the adsorption of biological molecules. These strategies are based on the adsorbent as well as the adsorption process. The strategies were:

1. Selecting the best adsorbent, based on selectivity, capacity and adsorption kinetics.

2. Improving adsorption performance of adsorbents by incorporation of small adsorbents in an alternative stationary phase; i.e. a particle loaded mixed matrix membrane (MMM).

3. Improving the selectivity of the adsorbent by molecular imprinting.

A systematic investigation of the chemical and physical structure of resin materials and their relation to adsorption properties was performed in Chapter 3. The separation of 5hydroxymethylfurfural (HMF) and glucose was studied for this purpose. The effect of the resins degree of crosslinking on the adsorption capacity (Chapter 3) and on the adsorption kinetics (Chapter 4) was also studied. MMMs were studied as alternative stationary phase for the adsorption of small molecules (Chapter 5 and Chapter 6). Different molecular weight molecules were studied for this purpose as well as HMF, that was already studied with a common stationary phase. Finally the molecular imprinting of a small acid was systematically investigating to improve the adsorption selectivity of adsorbents for small acids (Chapter 7).

The general conclusions with respect to resin selection, hypercrosslinking and mixed matrix membranes design are discussed, first followed by the discussion of the specific conclusions regarding furan separation from glucose and molecular imprinting of acid molecules.

\subsection{General conclusions}

\section{Systematic investigation of resin properties to identify the key properties}

Literature on furan removal from sugars was inconclusive with respect to the selection of best resin for this separation. In literature different resins were studied for this separation by adding them to a hydrolysate containing sugar and furans [1-3] or by performing adsorption experiments with furans 
[2, 4]. Unfortunately none of these studies systematically studied the effect of the material properties on the adsorption properties and it was therefore impossible to state which properties were key and how the adsorption performance could be improved. Although weak anion exchange groups are mentioned as good candidates for the separation, it is probably the non-polar styreneDVB matrix of the mentioned weak anion exchange resins that is responsible for furan adsorption $[5,6]$.

In chapter $\mathbf{3}$ of this study two styrene based neutral resins and three different styrene based anion exchange resins were investigated for the separation of the furan HMF from the sugar glucose. Insight in the adsorption mechanism is obtained by relating the adsorption isotherms of HMF and glucose (pure and competitive) to the physical (BET surface area, pore size, pore volume, swelling and water uptake) and chemical properties (none, weak or strong anion exchange groups) of the resins. HMF was adsorbed from water with all the studied styrene based (ion exchange) polymeric resins. For efficient removal, a high BET surface area of the resin is a key property. Although introduction of polar groups in the form of tertiary amines appears to increase the affinity of the resin material for HMF, these groups also introduce affinity for glucose resulting in loss of fermentable sugar. However the presence of glucose did not affect the HMF adsorption capacity and solubility and competition between HMF and glucose for adsorption sites was not observed in the ion exchange resins. HMF adsorption is likely established by $\pi-\pi$ interactions between the styrene matrix and the ring of the furan, while glucose adsorption is predominantly by hydrogen interactions with the polar groups of the ion exchange groups. The styrene based resin Dowex Optipore L-493 shows a high specificity for HMF adsorption while it does not exhibit specificity for glucose. This makes it an excellent adsorber for HMF removal from hydrolysate for the fermentation of glucose.

The investigation of the best adsorption resin for the separation of HMF from glucose showed that selecting the best resin for this and any other separation from a multi component mixture, requires systematic analysis of structure-property relationships for a variety of different resins. This investigation should include the physical properties (BET surface area, pore size, pore volume, swelling and solvent uptake) and chemical properties (e.g. chemical composition matrix) and relate them to the adsorption isotherms of the components of the mixture (pure and competitive). Only 
then is it possible to design the best resin based on adsorption thermodynamics and optimize the adsorption step for further reduction of capital and operation cost in the quest for cheaper, more sustainable and environmentally friendly bioproducts. This becomes even more important considering the vastly different furans that can be derived from biorenewable sugar based feedstocks.

\section{Hypercrosslinking resins for process intensification with a selective resin}

Chapter 3 described that Dowex Optipore L-493 (Optipore) had excellent adsorption properties with respect to adsorption thermodynamics. In Chapter 4 the kinetic properties of Optipore for the separation of HMF from glucose Optipore outperforms any other resin studied so far.

Optipore is a hypercrosslinked polymer [7, 8]. These polymers are prepared by emulsion polymerization and are further crosslinked while swollen in a good apolar solvent to increase the adsorption capacity. In contrast to conventional macroporous resins, these hypercrosslinked apolar resins swell in water even though they have high degrees of crosslinking [9]. This swelling in water is caused by the inner strain caused by the high degree of crosslinking, which is released by swelling of the polymer. The hypercrosslinked resins show higher internal surface areas and more uniform pore sizes with improved physical strength compared to macroporous resins. Chapter 3 and Chapter $\mathbf{4}$ show that this lead to superior adsorption capacities and adsorption kinetics.

Due to the fact that often polar solvents are used in biorefinery and the formed (by)products are usually less polar, the use of hypercrosslinked apolar resins in biorefinery processes offers great potential for cheaper, more sustainable and environmentally friendly bioproducts.

\section{Kinetic analysis of mixed matrix membranes with zero length column experiments}

Stirred or shaken vessels are most often used to determine the adsorption kinetics of adsorbents. Although they are also used to determine the kinetic performance of MMMs [10-12] they are not suitable for MMMs where flow though the membrane is applied since convective transport through the membrane is then absent in these measurements. A solution for this is offered by an alternative method, the zero length column (ZLC) introduced by Eic and Ruthven in 1988 and frequently used to study the adsorption kinetics of adsorbents [13]. This method ensures maximal convective flow through the pores of the membrane minimizing solute transport time by slow diffusion through the membrane pores when MMMs are placed in a filter holder. In Chapter 5 and Chapter 6 ZLC 
experiments were successfully used to study the adsorption rate of small solutes in MMM and we recommend the use of ZLC experiments to study the kinetic properties of MMMs for small solutes when used with convective flow through the membrane pores.

\section{Desorption}

An equally important factor in designing a good adsorbent and adsorption process is a fast release of the solute from the adsorber. Chapter 4 describes the desorption of HMF from the column. Although HMF is released from the column by rinsing it with water, desorption requires a high amount of water. Faster desorption of HMF at higher temperature indicates that desorption with steam could improve the release of HMF from the column. In literature the desorption is studied using different solvents (e.g. methanol, ethanol, n-propanol and n-butanol, acetone) [6, 14]. Although these solvents removed HMF effectively, their strong interaction with the resin, thus introducing another component in the system, is a major drawback.

Although most research on furan adsorption focusses on the adsorption properties of resins and the adsorption step in the process, the desorption step is an equally important factor. In considering an adsorption process, detailed analysis of the desorption (i.e. energy requirements and solvent use) is required to reflect on the economic feasibility of the adsorption process.

\section{MMMs for the adsorption of small solutes}

Conventional stationary phases such as adsorption columns, show large capacities but are generally not suitable for high linear velocities and therefore need large installations [15]. This is caused by the long diffusion distances in the resin. The transport of the solutes to the particles occurs through convective transport, while a considerable part of the adsorption time is determined by the slow intra particle diffusion in the particles [16]. Although smaller particles could be used, these result in very high pressure drops over the column, making this approach not suitable. A solution for this is the use of very small adsorption particles $(\sim 10 \mathrm{~nm})$ incorporated in so-called mixed matrix membranes (MMMs) $[17,18]$. The small particles reduce the diffusional path of the solute in the resin (and thereby the adsorption time) significantly, while the flow through the bed and along the particles is improved simultaneously because of the porous structure of the membrane. 
In Chapter 5 and Chapter 6 the incorporation of small resin particles in MMMs for the adsorption of small molecules was investigated. The adsorption capacities of the small resins were comparable to the adsorption capacities of the small resins incorporated in the MMMs. However due to the porous structure of the membranes, the resin volume per bed volume is much lower in the MMMs than in the packed bed. The total volume of MMMs should be larger to obtain comparable adsorption capacities.

Zero length column experiments showed that the overall diffusion (time to reach equilibrium adsorption) is much shorter for MMMs than for conventional large resins. In Chapter 6 the MMMs breakthrough curves were also measured and compared with the breakthrough curves obtained with a packed bed in Chapter 4. When the only the resin volume in the MMMs and the packed bed is considered, the resins in the MMMs perform much better than in the packed bed. For MMMs the breakthrough is later and improves with increasing flowrate and so does the column capacity. This is not the case for a conventional packed bed as was measured in Chapter 4. However, because the flowrate of packed bed and the MMMs were not optimized in Chapter $\mathbf{4}$ and Chapter $\mathbf{5}$, it is not possible to determine which type of system, packed bed or MMM, performs best. Conclusions on which system shows the lowest capital and operating cost requires a thorough investigation and comparison of both systems based on concentration of the solutes, flow rate, breakthrough points, pressure drops, mechanical strength, desorption characteristics, performance stability, maintenance and life time expectancy.

The comparison of breakthrough curves was performed with a resin that shows a very fast internal particle diffusion. Faster flow rates hardly reduces the performance of the packed bed and the use of MMMs is not persé advantageous. However experiments with a resin that shows very low internal particle diffusion show that the use of MMMs could be very profitable in that case (Appendix). Although we believe that investing in a good resin based on capacity and kinetic properties is the best strategy for a good adsorption process, this is not always possible. MMMs with could be a good alternative for a packed bed in such cases. 


\subsection{Case specific conclusions}

\section{HMF-glucose separations}

The separation of HMF from glucose is an important process in biorefinery. HMF is considered an important platform chemical which can be produced with a biorefinery process by dehydration of carbohydrates (e.g. glucose, fructose)[19]. It is also a toxic byproduct formed during the release of carbohydrates from lignocellulosic biomass to produce of ethanol [20]. As such the separation of HMF from glucose is an important separation process.

The research presented in Chapter $\mathbf{3}$ and Chapter $\mathbf{4}$ showed that the hypercrosslinked Optipore resin showed excellent adsorption properties that have not been met by resins studied so far, based on adsorption capacity as well as adsorption kinetics.

When the process is used to remove HMF as toxic byproducts, further research will have to show whether other toxins that are present in the fermentation broth can be removed in one step with one resin or a mixture with different resins or in multiple steps with multiple resins.

The Optipore resin has been incorporated in MMMs to improve the kinetic properties even further (Chapter 6). Although the kinetic performance appears to be improved, further research is required to conclude whether MMMs are a big improvement to the separation process. Conclusions on which system (packed bed or MMM) shows the lowest capital and operating cost requires a thorough investigation and comparison of both systems based on concentration of the solutes, flow rate, breakthrough points, pressure drops, mechanical strength, desorption characteristics, performance stability, maintenance and life time expectancy. However, without optimization hypercrosslinked resins in a packed bed already show excellent adsorption performance. It is very likely that after optimization of the process with the hypercrosslinked resin in a packed bed the adsoption performance of the packed bed cannot be matched by MMMs.

\section{Molecular imprinting to increase the selectivity of small acid adsorption}

Molecular imprinting of polymers has been considered by researchers to mimic the lock-and-key principle for affinity adsorbers. However molecular imprinting of small molecules for aqueous applications is not straightforward. The polymerization is difficult or impossible in water. Sorption from water often fails and non-specific hydrophobic adsorption is often strong which results in 
unselective adsorption. Research has shown that molecular imprinted polymers (MIP) that are selective for biomolecules in aqueous media need careful optimization in terms of functional monomer, cross linker, pH, buffer, solvent etc. However, often MIPs do not show selectivity in aqueous media at all [21].

In Chapter $\mathbf{7}$ the molecular imprinting of small acid molecule salicylic acid with pyridine was investigated. The research showed that the $\mathrm{pH}$ and the polarity of the solvent should be very carefully selected for molecular imprinting of this molecule. The continuous variation method showed that the optimal conditions of the solvent for complex formations were at low $\mathrm{pH}$ in a solvent that was as polar as possible. This is limited by the solubility of the monomers. Complex formation between the acid and the pyridine only occurs when both molecules are charged and ionic interactions are possible. Furthermore the $\mathrm{pH}$ necessary for rebinding is even lower due to a due to a local pH shift inside the membrane.

This presented research made an attempt towards molecular imprinting for the separation of small acid molecules. A molecular imprint of a small acid was recognized from an aqueous matrix by systematically investigating the molecular interactions of the acid-base complex. This research shows that molecular imprinting of small molecules with an acid group as functional imprinting group, is only possible when the conditions during polymerization as well as during sorption, are adjusted, in order for the acid and base to form a complex. Furthermore much higher selectivities are reached when organic solvents are used and it is not clear whether these will ever be reached for these separations without them. The stringent requirements of the adsorption matrix of MIPs only appear practical for clinical applications or on a very small scale. For the MIPS to become viable for biotechnology applications higher selectivity, lower demands of the adsorption matrix and better predictability of the MIP performance is necessary.

\subsection{Conclusion}

The basis for an effective adsorption process is an adsorbent that shows high selectivity for the separation and high capacity with fast adsorption kinetics. Finding this adsorbent requires a 
systematic investigation of the chemical and physical properties of the available adsorbent and the effect of these properties on the adsorption process. In considering an adsorption process, detailed analysis of the desorption (i.e. energy requirements and solvent use) is required to reflect on the economic feasibility of the adsorption process.

Molecular imprinting promises an easy solution to improve the selectivity in adsorption processes. However molecular imprinting is not straightforward for small molecules and big improvements are not foreseen in the near future.

MMM show fast overall adsorption kinetics for small molecules without the loss of adsorption capacity nor with the high pressure drops and channeling associated with packed bed with small particles. MMMs therefore allow very fast adsorption at very high flow rates and could therefore play a valuable role in the high throughput/high volume applications in downstream processing.

Due to the fact that often polar solvents are used in biorefinery and the formed (by)products are usually less polar, the use of the superior hypercrosslinked apolar resins in biorefinery processes offers great potential for cheaper, more sustainable and environmentally friendly bioproducts.

\subsection{References}

1. Maciel de Mancilha, I. and M.N. Karim, Evaluation of ion exchange resins for removal of inhibitory compounds from corn stover hydrolyzate for xylitol fermentation. Biotechnology progress, 2003. 19(6): p. 1837-1841.

2. de Carvalho, W., et al., Detoxification of sugarcane bagasse hemicellulosic hydrolysate with ion-exchange resins for xylitol production by calcium alginate-entrapped cells. Journal of Chemical Technology \& Biotechnology, 2004. 79(8): p. 863-868.

3. Vern, C., et al., The beet sugar factory of the future. International Sugar Journal, 1995. 97(1159): p. 310-316.

4. Nilvebrant, N.-O., et al., Detoxification of lignocellulose hydrolysates with ion-exchange resins. Applied Biochemistry and Biotechnology, 2001. 91-93(1-9): p. 35-49.

5. Fargues, C., R. Lewandowski, and M.-L. Lameloise, Evaluation of ion-exchange and adsorbent resins for the detoxification of beet distillery effluents. Industrial \& Engineering Chemistry Research, 2010. 49(19): p. 9248-9257.

6. Weil, J.R., et al., Removal of fermentation inhibitors formed during pretreatment of biomass by polymeric adsorbents. Industrial \& Engineering Chemistry Research, 2002. 41(24): p. 6132-6138.

7. Xu, Z., Q. Zhang, and H.H.P. Fang, Applications of Porous Resin Sorbents in Industrial Wastewater Treatment and Resource Recovery. Critical Reviews in Environmental Science and Technology, 2003. 33(4): p. 363-389.

8. Tsyurupa, M.P. and V.A. Davankov, Porous stucture of hypercrosslinked polystyrene: Stateof-the-art mini-review. Reactive and Functional Polymers, 2006. 66(7): p. 678-779. 
9. Yan, J., X. Wang, and J. Chen, Swelling of porous styrene-divinylbenzene copolymers in water. Journal of Applied Polymer Science, 2000. 75(4): p. 536-544.

10. Avramescu, M.-E., Z. Borneman, and M. Wessling, Mixed-matrix membrane adsorbers for protein separation. Journal of Chromatography A, 2003. 1006(1-2): p. 171-183.

11. Saiful, Z. Borneman, and M. Wessling, Enzyme capturing and concentration with mixed matrix membrane adsorbers. Journal of Membrane Science, 2006. 280(1-2): p. 406-417.

12. Tetala, K.K.R., et al., A metal ion charged ixed matrix membrane for selective adsorption of hemoglobin. Separation and Purification Technology, 2013. 115(0): p. 20-26.

13. Eic, M. and D.M. Ruthven, A new experimental technique for measurement of intracrystalline diffusivity. Zeolites, 1988. 8(1): p. 40-45.

14. Nanoti, A., et al., Process studies for development of adsorption technology for furfural recovery from waste water. Environmental Progress, 2008. 27(3): p. 308-312.

15. Avramescu, M.-E., Z. Borneman, and M. Wessling, Membrane chromatography, in Handbook of Membrane Separations; Chemical, Pharmaceutical, Food and Biotechnological Applications, A.K. Pabby, S.S.H. Rizvi, and A.M. Sastre, Editors. 2008, CRC Press.

16. Ghosh, R., Protein separation using membrane chromatography: Oportunities and challenges. Journal of Chromatography A, 2002. 952(1-2): p. 13-27.

17. Orr, V., et al., Recent advances in bioprocessing application of membrane chromatography. Biotechnology Journal, 2013. 31(4): p. 450-465.

18. Saiful, Mixed matrix membrane adsorbers for protein and blood purification, 2007, University of Twente: Enschede.

19. Detoni, C., et al., Selective Liquid Phase Adsorption of 5-Hydroxymethylfurfural on Nanoporous Hyper-Cross-Linked Polymers. ACS Sustainable Chemistry \& Engineering, 2014. 2(10): p. 2407-2415.

20. Huang, H.J., et al., A review of separation technologies in current and future biorefineries. Separation and Purification Technology, 2008. 62(1): p. 1-21.

21. Zhang, H., Water-compatible molecularly imprinted polymers: Promising synthetic substitutes for biological receptors. Polymer, 2014. 55(3): p. 699-714. 


\section{Appendix: Particle loaded mixed matrix membranes for resins with poor kinetic adsorption properties}

The 5-hydroxymethylfurfural (HMF) breakthrough and desorption curves were determined as described in Chapter 4 and Chapter 6 with a packed bed with Lewatit MP62 resins (MP62) and the MMMs from Chapter 5 fabricated with MP62 (Figure 1 and Figure 2).

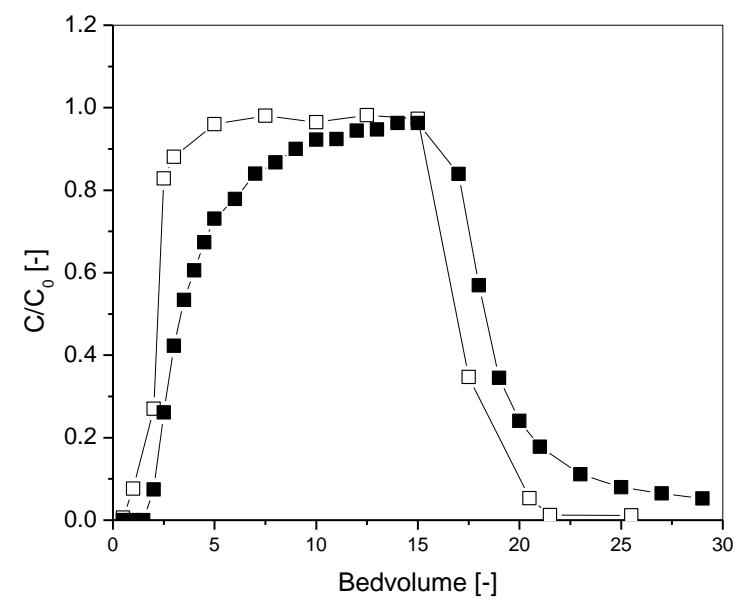

Figure 1: HMF breakthrough curves at $20^{\circ} \mathrm{C}, 5000 \mathrm{mg} / \mathrm{l} \mathrm{HMF:}$ - MMM, $8 \mathrm{BV} / \mathrm{h}$; $\square$ packedbed, $8 \mathrm{BV} / \mathrm{h}$.

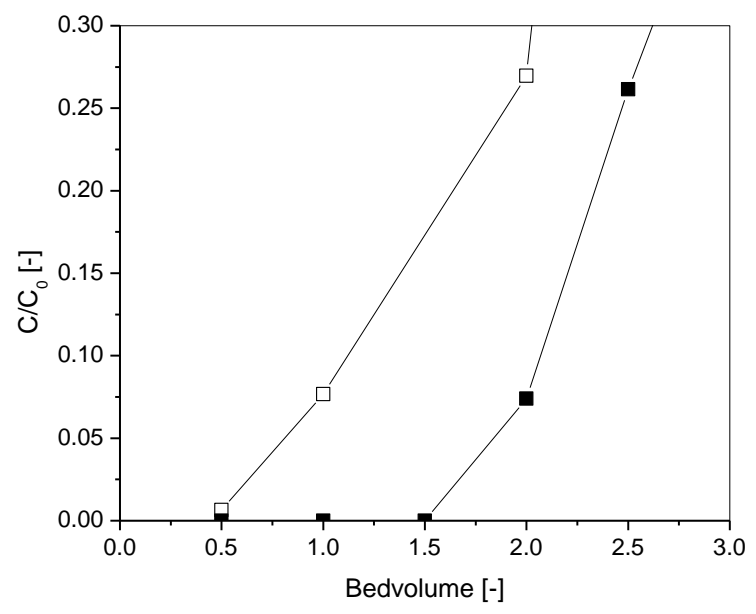

Figure 2: Detail of the HMF breakthrough point of Figure 1: - MMM, $8 \mathrm{BV} / \mathrm{h}$; $\square$ packedbed, $8 \mathrm{BV} / \mathrm{h}$. 
Although the MP62 resin shows reasonable HMF adsorption capacities in batch adsorption experiments (Chapter 3), the packed bed breakthrough curve (Figure 1 and 2, open symbols) shows that the adsorption kinetics of the resin are very poor. With a flowrate of $8 \mathrm{BV} / \mathrm{h}$ HMF is not or hardly adsorbed, shown by the immediate and complete breakthrough of HMF. However when the resin size is reduced and the resin is incorporated in the MMM, much more HMF adsorption is observed (Figure 1 and 2, closed symbols). Although the adsorption performance is still inferior to the performance of Dowex Optipore L-493 (Chapter 4 and Chapter 6), it shows that the use of MMMs could be very advantageous for resins with slow internal diffusion properties, as was suggested in Chapter 6 . 


\section{Summary}

In this thesis we studied several strategies to improve adsorption technology for the adsorption of biobased molecules. These strategies are based on the adsorbent as well as the adsorption process.

A systematic investigation of the chemical and physical structure of resin materials and their relation to adsorption properties was performed in Chapter 3. The separation of 5hydroxymethylfurfural (HMF) from glucose was studied for this purpose with two neutral styrene based resins and three different styrene based anion exchange resins. The systematic study showed that HMF adsorption is likely established by $\pi-\pi$ interactions between the styrene matrix and the ring of the furan, while glucose adsorption is predominantly by hydrogen interactions with the polar groups of the ion exchange groups. This study determined that a high BET surface area of the resin is a key property to obtain high adsorption capacities. The styrene based neutral resin Dowex Optipore L-493 (Optipore) showed a high specificity and capacity for HMF adsorption while it did not exhibit specificity for glucose. This makes it an excellent adsorber for HMF removal from hydrolysate for the fermentation of glucose.

In Chapter $\mathbf{4}$ the kinetic properties of Optipore were examined with a zero length column method and breakthrough experiments for the separation of HMF from glucose. The zero length column experiments indicated that the diffusion of HMF is lower in a glucose/water solution than in water caused by the increased viscosity of the solution. The efficiency of the resin was further studied with breakthrough experiments in a packed bed. The breakthrough curves of HMF on Optipore showed that the column is very efficient at the conditions tested. The breakthrough curves were compared to breakthrough curves of other resins in literature and Optipore outperformed them all. Desorption of HMF from the column was also studied. Desorption of HMF from the column was also studied and this showed to be challenging. At $20^{\circ} \mathrm{C}$ only $81 \%$ of the HMF was removed after 30 bed volumes. Other options for HMF desorption were discussed, but this shows that although Optipore is a very promising candidate for HMF adsorption, desorption needs further investigation.

In Chapter 5 the incorporation of small resin particles in MMMs for the adsorption of anions with biological origin at $\mathrm{pH} 7$ was investigated. The adsorption system was studied for the adsorption of 
folic acid (FA, $441 \mathrm{~g} / \mathrm{mol}$ ), a peptide (PEP, $1028 \mathrm{~g} / \mathrm{mol}$ ) and bovine serum albumin (BSA, 64.000 $\mathrm{g} / \mathrm{mol}$ ). The adsorption mechanism was studied and the adsorption of the anions on MP62 at $\mathrm{pH} 7$ is achieved by ion exchange. The adsorption capacity and adsorption kinetics of FA, PEP and BSA at pH 7 on MP62 resins $(625 \mu \mathrm{m}$ and $8 \mu \mathrm{m})$ and MP62 $(8 \mu \mathrm{m})$ loaded MMM's was studied with adsorption isotherms. MMMs loaded with small ion exchange particles showed similar adsorption capacities as the pure small ion exchange particles and the matrix of the membrane does not affect the adsorption capacity. It even appears that by incorporation of the particles in the MMM, agglomeration of the small resins is prevented. Zero length column experiments showed that the incorporation of small particles in MMMs reduces the overall adsorption rate although the apparent effective diffusion coefficient of MMMs is larger than the effective diffusion coefficient of the resins. This study showed that MMMs show fast overall adsorption kinetics without loss of resin capacity nor high pressure drops and channeling. MMMs therefore allow very fast adsorption at very high flow rates and could therefore play a valuable role in downstream processing.

In Chapter 6 the incorporation of small Optipore particles in MMMs for the adsorption of HMF was investigated. The adsorption capacities of the original resin $(557 \mu \mathrm{m})$ small resin $(15 \mu \mathrm{m})$ and MMM containing the same resin $(15 \mu \mathrm{m})$ were studied with adsorption isotherms. All adsorbents (small, large and $\mathrm{MMM}$ ) show comparable adsorption capacities. This shows that the fabrication method of the MMMs does not block adsorption sites and allows for the full use of the particle capacity. The effective diffusion coefficient of Optipore for HMF was studied with zero length column experiments. These experiments show that the effective diffusion coefficient is only slightly reduced when particles are incorporated in the membranes, while the overall adsorption time is improved enormously. This is reflected in the fast flow rates that could be used when the breakthrough curves were determined. The breakthrough curves also show that the adsorption capacity of the resins themselves is even improved when the membranes are used compared to a packed bed with large resins. Due to the open structure of the MMMs, the MMMs show improved adsorption capacity and fast adsorption rates at high flow rates, which allows for high throughput application of MMMs for HMF separation. Furthermore this research shows that MMMs could be a good alternative for traditional packed beds for the adsorption of small molecules. 
Molecular imprinting is a technique to create adsorbers with tailored adsorption sites for specific molecules. In Chapter $\mathbf{7}$ molecular imprinting of a small acid, salicylic acid (SaA) was studied with vinylpyridine (4VP) as functional monomer and diethyleneglycoldimethacrylate (DEGDM) as crosslinker. The study showed that especially the composition of the solvent and $\mathrm{pH}$ is important for successful molecular imprinting of SaA. Optimal solvent composition and $\mathrm{pH}$ was determined with the continuous variation method. With this method it is possible to see if a complex between target molecule and a functional monomer is formed at a certain solvent composition and if so, the ratio of target molecule to the functional monomer in the complex. The continuous variation method showed that imprinting can be achieved at low $\mathrm{pH}$ in a solvent that mimics the aqueous environment. Molecular imprinting with a high crosslinker to polymer ratio showed to lower the $\mathrm{pH}$ inside the membrane compared to the $\mathrm{pH}$ of the bulk solution. Consequently the charge inside the membrane and its adsorption behavior was different than expected. The molecular imprinted membranes investigated show clear $\mathrm{pH}$ transitions and fast kinetics. Consequently, molecular imprinting of membranes (MIMs) seems to be a very interesting technology for the selective recovery or separation of specific molecules from complex feed mixtures and therefore the downstream processing in biorefinery. However the membranes showed very stringent requirements regarding the sorption conditions which is in conflict with the process streams in the downstream processing of biorefinery products.

The last part of this thesis, Chapter 8 discusses the potential of the hypercrosslinked adsorber Optipore and mixed matrix membranes for the separation of HMF and molecules with biological origin in general. It briefly presents the main insights that were experienced in this study and it indicates the directions for further optimization, design and development of the studied technologies for cheaper, more sustainable and environmentally friendly bioproducts 


\section{Samenvatting}

In dit proefschrift zijn verschillende strategieën bestudeerd om de adsorptietechnologie van moleculen van biologische origine te verbeteren. De studie richtte zich op zowel de adsorbent als de adsorptie technologie.

In Hoofdstuk 3 zijn de chemische en de fysische eigenschappen van de adsorptie resins bestudeerd en gerelateerd aan de adsorptie eigenschappen. De scheiding van 5hydroxymethylfurfural (HMF) van glucose is onderzocht met twee neutrale resins met een styreen matrix en drie anionwisselaars, ook met een styreen matrix. Systematisch onderzoek liet zien dat HMF adsorptie plaatsvindt door de $\pi-\pi$ interactie tussen de styreen matrix en de ring van de furan. Glucose adsorptie vindt hoofdzakelijk plaats door de vorming van waterstofbruggen met de polaire groepen van de ionenwisselaars. Het onderzoek toont aan dat een hoog BET oppervlak van het resin essentieel is voor een hoge adsorptie capaciteit. Het neutrale resin Dowex Optipore L-493 heeft een hoge selectiviteit en capaciteit voor HMF terwijl het geen glucose adsorbeert. Hierdoor is het een uitstekende kandidaat voor de scheiding van HMF en glucose.

In Hoofdstuk 4 zijn de kinetische eigenschappen van Optipore onderzocht met zero length kolom en breakthrough experimenten voor de scheiding van HMF van glucose. De zero length kolom experimenten lieten zien dat de diffusie van HMF in glucose/water lager is dan in water. Dit komt door de verhoogde viscositeit van de water/glucose oplossing ten opzichte van water. De efficiëntie van de scheiding werd verder onderzocht door middel van breakthrough experimenten in een gepakt bed. De breakthrough curves lieten zien dat Optipore in een gepakt bed HMF heel efficiënt scheidt van glucose. De breakthrough curves zijn vergeleken met breakthrough curves van andere resins en Optipore presteert veel beter dan die resins met betrekking tot adsorptiecapaciteit en adsorptie kinetiek.. Desorptie van HMF is ook bestudeerd en dat blijkt een uitdaging met dit resin. Bij kamertemperatuur zijn er 30 bedvolumes water nodig om 80\% van de HMF te verwijderen van de kolom. Andere opties voor desorptie werden ook besproken in het hoofdstuk, maar het onderzoek naar de desorptie laat zien dat, ondanks dat Optipore een hele goede adsorbent is voor HMF, er nog meer onderzoek nodig is naar de desorptie voordat een economisch rendabel proces mogelijk is met dit resin. 
In Hoofdstuk $\mathbf{5}$ is de toepasbaarheid van mixed matrix membranen voor de scheiding van anionen met biologische oorsprong bestudeerd bij pH 7. Hiervoor zijn de volgende anionen gekozen: foliumzuur (FA, $441 \mathrm{~g} / \mathrm{mol}$ ), een peptide (PEP, $1028 \mathrm{~g} / \mathrm{mol}$ ) en bovine serum albumin (BSA, 64.000 $\mathrm{g} / \mathrm{mol}$ ). Het adsorptie mechanisme van de anionen op de anionenwisselaar MP62 bij pH 7 vindt plaats door ionwisseling. De adsorptiecapaciteit en adsorptie kinetiek van FA, PEP and BSA bij pH 7 op MP62 resins $(625 \mu \mathrm{m}$ and $8 \mu \mathrm{m})$ and MP62 $(8 \mu \mathrm{m})$ geladen MMM's is bestudeerd met adsorptie isothermen. MMMs laat dezelfde adsorptiecapaciteit zien als de kleine adsorptie resins, wat aantoont dat de matrix van het membraan de adsorptie capaciteit niet beïnvloedt. Het lijkt er zelfs op dat agglomeratie van de kleine resins voorkomen wordt door incorporatie van de resins in MMMs. Zero length kolom experimenten laten zien dat ondanks dat de effectieve diffusiecoëfficiënt van de resins in MMMs lager is dan van de pure resins, de overall adsorptietijd lager is in MMMs. In dit hoofdstuk is aangetoond dat MMMs hele snelle overall adsorptiekinetiek laten zien zonder verlies van capaciteit en zonder dehoge drukval en kanaalvorming die normaalgesproken gezien wordt bij het gebruik van kleine resins. MMMs maken daardoor snelle adsorptie en hoge flowsnelheden mogelijk en zijn mogelijk goed te gebruiken bij downstream processen.

In Hoofdstuk 6 zijn kleine Optipore resins verwerkt in MMMs voor de adsorptie van HMF. The adsorptiecapaciteit van het originele resin $(557 \mu \mathrm{m})$ het kleine resin $(15 \mu \mathrm{m})$ en de MMM met het kleine resin $(15 \mu \mathrm{m})$ werden bestudeerd met adsorptie isothermen. Alle adsorbents laten vergelijkbare adsorptiecapaciteiten zien. Dit toont aan dat de fabricagemethode van de MMMs geen effect heeft op de capaciteit van de resins. De effectieve diffusiecoëfficiënt van HMF in Optipore is bestudeerd met zero length column experimenten. Deze experimenten laten zien dat de effectieve diffusiecoëfficiënt maar een klein beetje lager is als de deeltjes in een MMM zitten terwijl de overall adsorptietijd enorm verbeterd is. Dit was ook duidelijk uit de hoge flowsnelheden die gebruikt konden worden met MMMs bij de breakthrough experimenten. The breakthrough curves lieten ook zien dat de capaciteit van de resins verbetert als de membranen gebruikt worden in een gepakt bed opstelling ten opzichte van een conventioneel gepakt bed. Dit hoofdstuk heeft aangetoond dat de open structuur van MMMs hele hoge flowsnelheden mogelijk zijn waardoor deze membranengeschikt zijn voor bulk toepassingen in het downstream proces. Verder heft dit onderzoek aangetoond dat MMMs een goed alternatief kunnen zijn voor traditionele gepakte bedden voor de scheiding van kleine moleculen. 
Moleculair inprinten is een techniek die gebruikt wordt om hele selectieve adsorptiesites te maken voor specifieke moleculen. In Hoofdstuk 7 is salicylzuur (SaA) moleculair geinprint met 4vinylpyridine (4VP) met diethyleenglycoldimethacrylaat (DEGDM) als crosslinker. Deze studie heft aangetoond dat de compositie en $\mathrm{pH}$ van het oplosmiddel heel belangrijk is voor het moleculair inprinten. De beste samenstelling van het oplosmiddel is onderzocht met behulp van de continue variatie methode. Met deze methode is kan het eventueel gevormde complex in verschillende oplosmiddelen aangetoond worden. Als een complex aanwezig is kan ook de ratio tussen molecuul en functioneel monomeer in het complex bepaald worden. Met behulp van de continue variatie methode werd aangetoond dat inprinten van $\mathrm{SaA}$ alleen bereikt kan worden bij lage $\mathrm{pH}$ in een oplosmiddel dat lijkt op een waterig milieu. Door het moleculair imprinten met een hoge crosslinker ratio wordt de $\mathrm{pH}$ in het membraan ten opzichte van de bulkoplossing veel lager. Hierdoor was de lading van het membraan en de adsorptie anders dan verwacht. De bestudeerde geinprinte membranen hebben een duidelijke $\mathrm{pH}$ overgang en hebben ook een snelle adsorptie kinetiek. Hierdoor lijken ze heel geschikt voor de selectieve scheiding van specifieke moleculen uit een complex mengsel. Helaas zijn voor adsorptie hele specifieke eigenschappen nodig die conflicteren met de eigenschappen van de processtromen van biorefinery producten.

Het laatste deel van het proefschrift, Hoofdstuk 8, reflecteert op de mogelijkheden van de hypercrosslinked adsorber Optipore en MMMs voor de scheiding van HMF en moleculen van biologische origine in het algemeen. Het beschrijft kort de resultaten die gevonden zijn tijdens het onderzoek en geeft aan welke richtingen verder onderzoek nodig hebben om goedkopere, duurzamere en milieuvriendelijkere bioproducten te produceren. 


\section{Dankwoord}

Met dit stukje sluit ik mijn proefschrift af. Letterlijk omdat het echt aan het eind van het boekje staat en figuurlijk, omdat het echt het allerlaatste is wat ik schrijf.

En dat mocht ook wel eens een keer.

Voor de meeste van jullie zal dit stukje het enige zijn wat jullie lezen. Ik beloof jullie dat de introductie en de Nederlandse samenvatting ook best behapbaar zijn.

Als eerste wil ik graag Matthias Wessling bedanken. Helaas won je tijdens mijn promotie een prestigieuze prijs en ging RWTH Aachen er met je vandoor. Gelukkig was je nog wel regelmatig beschikbaar voor een kort overleg. De discussies die we tijdens mijn promotie hadden over mijn werk waren prikkelend en daagden me uit. In de afrondende fase heb je me ook goed bijgestaan en motiveerde je me enorm. Dank je wel!

Kitty Nijmeijer heeft me wegwijs gemaakt in de onderzoekswereld. Ze heeft met name het begin van het project vorm gegeven en had de ondankbare taak bijna het hele proefschrift te corrigeren. Het was niet makkelijk, maar we staan hier nu wel allebei. Ik heb veel van je geleerd.

Mijn leukste onderzoekstijd was terwijl Erik Vriezekolk zijn masteropdracht deed als onderdeel van het onderzoek. Tegelijkertijd werkte Erik Rolevink als technicus aan het project en later deed Sander Veltkamp ook nog een deel van het onderzoek. Het was geweldig om met jullie te werken. Die tijd stond bol van de interactie, goede ideeën, leuke discussies en een biertje op zijn tijd. Het was super en ik heb veel aan jullie gehad.

Ik wil graag ISPT bedanken voor het mogelijk maken van mijn project. De meetings bij jullie waren leuk en waardevol. De medewerkers van Purac die betrokken waren bij het project en met name Tanja Djekic, wil ik hartelijk bedanken voor de waardevolle discussies, het advies en het werk dat jullie gedaan hebben. Christine Wegman wil ik hartelijk bedanken voor haar hulp met de HSDM.

Beste collega's en allerbeste kamergenoten (Jigar, Matias, Bernke, Sandra, Jeroen, Jumeng, Beata) van MTG. Ik heb 4 jaar van alles met jullie beleefd: Ups-and-downs. Bedankt voor alle ups en de steun bij de downs. Sandra and Geraldine I always had a blast with anything we did, thank you so much for the great time! Greet Kamminga, je bent een hele bijzondere vrouw, ontzettend bedankt voor alles. Harmen, Antoine, Olga, Jeroen en Beata, bedankt voor alle gezelligheid, hulp in het lab. Herman bedankt voor de SEM hulp. MTP, Clemens, Genevieve, Joost en Julius Vancso, bedankt voor het warme welkom in jullie groep.

Al mijn roeiploeg genootjes (Marthe, Anke, Hilde Anita, Annemieke, Michelle, Jorien, Gerdine, Marloes, Geertje, Still Going Strong) bedankt voor alle fysieke afleiding en super tijd aan wal! 
Lieve Femmeusjes (Marleen, Agnes, Carla, Annemarie, Esther, Gwendy, Jiska, Marianne, Marjolein, Marloes en Tessa) jullie zijn inspirerende topvrouwen. Nog eentje dan!

Kim en Timo, jullie zijn gewoon leuk, lief en gezellig. Bernd en Francesca, I don't know how you manage to always create a party out of nothing. Never a dull moment. Love you guys!

Jorien en Gerdine, we kennen elkaar al bijna vanaf het begin van mijn studie. Toen waren we heel erg druk met het spotten van mooie mannen op de Bosbaan hard roeien. Inmiddels zijn we jaren verder, groter, wijzer en vele verhalen verder. Vaak heel gezellig, mooi en inspirerend, soms heel verdrietig en moeilijk. Jullie waren er echt altijd en ik kan jullie niet genoeg bedanken daarvoor.

Wilco: lief, leuk, bijzonder, ambitieus, rots in de branding, slim en excentriek. Altijd in voor een verassing en avontuur. Op naar meer! Marnix, een groot deel van dit boekje is geschreven met jou in mijn buik of daarop. Gelukkig weet je daar helemaal niks van. Je bent een geweldig mannetje en dat is genoeg.

Zonder mijn ouders zou ik nu niet zijn wie ik was. Bedankt voor jullie warme opvoeding. Ik heb een geweldige jeugd gehad met volop avontuur en geborgenheid. De herinneringen daaraan koester ik. 